$\operatorname{Argonne} \underset{\bigotimes}{\bigotimes}$

\title{
Annual Report of Monitoring at Morrill, Kansas, in 2011
}

\section{Environmental Science Division}


About Argonne National Laboratory

Argonne is a U.S. Department of Energy laboratory managed by UChicago Argonne, LLC under contract DE-AC02-06CH11357. The Laboratory's main facility is outside Chicago, at 9700 South Cass Avenue, Argonne, Illinois 60439. For information about Argonne and its pioneering science and technology programs, see www.anl.gov.

\section{Availability of This Report}

This report is available, at no cost, at http://www.osti.gov/bridge. It is also available on paper to the U.S. Department of Energy and its contractors, for a processing fee, from:

U.S. Department of Energy

Office of Scientific and Technical Information

P.O. Box 62

Oak Ridge, TN 37831-0062

phone (865) 576-8401

fax (865) 576-5728

reports@adonis.osti.gov

\section{Disclaimer}

This report was prepared as an account of work sponsored by an agency of the United States Government. Reference herein to any specific commercial product, process, or service by trade name, trademark, manufacturer, or otherwise, does not necessarily constitute or imply its endorsement, recommendation, or favoring by the United States Government or any agency thereof. The views and opinions of document authors expressed herein do not necessarily state or reflect those of the United States Government or any agency thereof, Argonne National Laboratory, or UChicago Argonne, LLC. 


\section{Annual Report of Monitoring at Morrill, Kansas, in 2011}

by

Applied Geosciences and Environmental Management Section Environmental Science Division, Argonne National Laboratory

March 2012

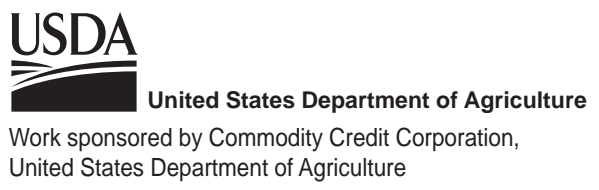




\section{Contents}

Notation.

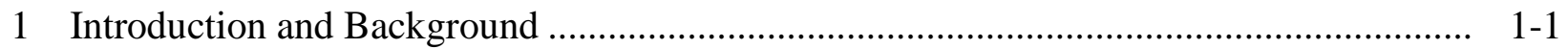

2 Sample Collection and Analysis Activities..................................................................... 2-1

2.1 Measurement of Groundwater Levels................................................................... 2-1

2.2 Monitoring Well and Private Well Sampling and Analyses..................................... 2-1

2.3 Surface Water and Sediment Sampling and Analyses.............................................. 2-2

2.4 Vegetation Sampling and Analyses ........................................................................... 2-3

2.5 Handling and Disposal of Investigation-Derived Waste ........................................... 2-4

2.6 Quality Control for Sample Collection, Handling, and Analysis .............................. 2-4

3 Results and Discussion .........................................................................................

3.1 Groundwater Level Data...................................................................................... 3-1

3.2 Groundwater Analysis Results.............................................................................. 3-1

3.3 Surface Water and Sediment Analysis Results ......................................................... 3-2

3.4 Vegetation Analysis Results and Observations ..................................................... 3-2

3.5 Comparison of Analytical Results for Samples Collected after Low-Flow Purging and after Purging of Three Well Volumes .................................................. 3-3

4 Conclusions and Recommendations ....................................................................... 4-1

4.1 Conclusions .................................................................................................... 4-1

4.2 Recommendations............................................................................................... 4-2

4.2.1 Groundwater Monitoring Frequency …………............................................ 4-2

4.2.2 Vegetation Sampling Frequency................................................................ 4-3

4.2.3 Sampling Methods ..................................................................................... 4-3

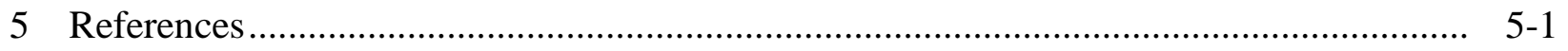

Appendix A: $\quad$ Sampling Activities at Morrill in 2011 ........................................................ A-1

Appendix B: $\quad$ Results from the AGEM Laboratory for Dual Analyses of Samples Collected at Morrill in 2011 and for Quality Control Samples ...................... B-1

Supplement 1: Waste Characterization Data...................................................................... CD

Supplement 2: Sample Documentation from TestAmerica Laboratories, Inc., for Groundwater Verification Samples.............................................................. on CD 


\section{Figures}

1.1 Location of Brown County and Morrill, Kansas .................................................. 1-4

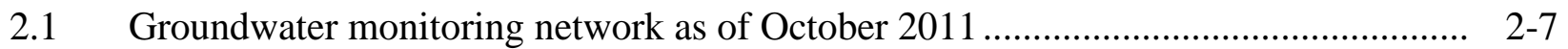

2.2 Locations of surface water and creek bed sediment sampling along

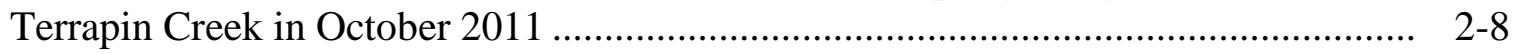

2.3 Locations of native vegetation sampling downgradient of the former CCC/USDA facility and along Terrapin Creek in July 2011 .................................. 2-9

3.1 Potentiometric surface, based on water levels measured manually on January 5, 2012

3.2 Hydrographs summarizing results of long-term water level monitoring from January 1, 2011, to December 31, 2011.............................................................. 3-26

3.3a Carbon tetrachloride concentrations in groundwater, April 2011 ........................... 3-27

3.3b Carbon tetrachloride concentrations in groundwater, October 2011 ........................ 3-28

3.4a Carbon tetrachloride concentrations in groundwater, April 2010 ............................ 3-29

3.4b Carbon tetrachloride concentrations in groundwater, September 2010 ..................... 3-30

3.5 Carbon tetrachloride concentrations in vegetation, July 2011 ............................... 3-31

4.1 Carbon tetrachloride concentrations in groundwater in October of 2003, 2007, and 2011 


\section{Tables}

3.1 Hand-measured groundwater levels in 2011

3.2 Results of analyses at the AGEM Laboratory for volatile organic compounds in groundwater samples collected in 2003-2011.

3.3 Field measurements for groundwater samples collected in 2003-2011.....

3.4 Results of analyses at the AGEM Laboratory for volatile organic compounds in surface water and sediment samples collected in 2007-2011

3.5 Results of analyses at the AGEM Laboratory for carbon tetrachloride and chloroform in vegetation samples collected in 2006-2011

4.1 Analytical results for carbon tetrachloride in groundwater samples collected at wells MW7W, MW8S, MW9S, and MW11S in September or October of years 2005-2011

A.1 Sequence of sampling activities in 2011

B.1 Analytical results from the AGEM Laboratory for quality control samples collected to monitor sample collection and handling activities in 2011

B.2 Analytical results from the AGEM Laboratory for dual analyses of samples collected in 2011

B.3 Analytical results from the AGEM Laboratory and TestAmerica for groundwater samples collected in 2011 and submitted for verification analysis.. 


\section{Notation}

AGEM Applied Geosciences and Environmental Management

AMSL above mean sea level

BGL below ground level

${ }^{\circ} \mathrm{C}$ degree(s) Celsius

CCC Commodity Credit Corporation

CD compact disc

DO dissolved oxygen

EPA U.S. Environmental Protection Agency

$\mathrm{ft} \quad$ foot (feet)

gal gallon(s)

hr hour(s)

in. inch(es)

KDHE Kansas Department of Health and Environment

L liter(s)

$\mu \mathrm{g} / \mathrm{kg} \quad$ microgram(s) per kilogram

$\mu \mathrm{g} / \mathrm{L} \quad$ microgram(s) per liter

$\mu \mathrm{S} / \mathrm{cm} \quad$ microsiemen(s) per centimeter

$\mathrm{mg} / \mathrm{L} \quad$ milligram(s) per liter

mi mile(s)

min minute(s)

$\mathrm{mV} \quad$ millivolt(s)

ORP oxidation-reduction potential

SOP standard operating procedure

TOC top of casing

USDA U.S. Department of Agriculture

VOC volatile organic compound 


\section{Annual Report of Monitoring at Morrill, Kansas, in 2011}

\section{Introduction and Background}

Carbon tetrachloride contamination in groundwater at Morrill, Kansas, was initially identified in 1985 during statewide testing of public water supply wells for volatile organic compounds (VOCs). High levels of nitrate were also present in the wells. The city of Morrill is located in Brown County in the northeastern corner of the state, about 7 mi east of Sabetha (Figure 1.1). The population of Morrill as of the 2010 Census was approximately 230 (down from 277 in 2000). All residents of Morrill now obtain their drinking water from the Sabetha municipal water system via a pipeline constructed in 1991.

From 1922 to 1991, eight different public water supply wells served the Morrill municipal system. Because of poor water quality, use of the various public wells was discontinued over time. The water quality problems included high nitrate levels attributed to numerous animal feeding operations in the vicinity; application of fertilizer to agricultural lands; excessive hardness; and elevated iron, sulfate, and total dissolved solids concentrations (above acceptable levels). The use of the local groundwater from any public well for municipal supply purposes was terminated in 1991 at the direction of the Kansas Department of Health and Environment (KDHE), and water was obtained from the Sabetha municipal water system.

Investigations of the carbon tetrachloride and nitrate contamination by the KDHE in 1989, 1994, and 1996 (KDHE 1989; GeoCore 1994a-d, 1996) identified a localized plume of carbon tetrachloride in groundwater extending downgradient from a grain storage facility in the northwestern section of Morrill. The facility was formerly operated by the Commodity Credit Corporation (CCC), an agency of the U.S. Department of Agriculture (USDA), from 1950 to 1971. After termination of the CCC/USDA grain storage operations in 1971, the property and existing grain bins continued to be used for private grain storage, up to the present time. Prior to 1986, commercial grain fumigants were commonly used by the CCC/USDA, as well as private and commercial grain storage operations, to preserve grain.

Because the identified carbon tetrachloride contamination could, in part, be potentially linked to historical use of carbon tetrachloride-based fumigants at its former facility, in 2003 the CCC/USDA assumed responsibility for the site investigation of the carbon tetrachloride contamination. The CCC/USDA involvement began with development and implementation of a 
work plan for a Phase I expedited site characterization (Argonne 2003). That investigation and subsequent investigations (Argonne 2004, 2005a) were performed by the Environmental Science Division of Argonne National Laboratory.

The initial investigation by the CCC/USDA in 2003 determined that soils at the former facility were not impacted by grain fumigation activities. Neither carbon tetrachloride nor chloroform was detected in near-surface soils or in subsurface soils collected to bedrock. Therefore, no identifiable human health risk is associated with either carbon tetrachloride or chloroform in shallow soils, which additionally pose no further threat of contamination to groundwater.

High carbon tetrachloride concentrations in groundwater (maximum $390 \mu \mathrm{g} / \mathrm{L}$ in a sample collected from monitoring well MW3S - located on the former CCC/USDA property in 1995) have declined significantly during long-term monitoring conducted earlier by the KDHE and currently by the CCC/USDA. The present maximum levels of $<50 \mu \mathrm{g} / \mathrm{L}$ confirm that no continuing soil source remains at the former CCC/USDA facility. Nevertheless, carbon tetrachloride concentrations exceeding the KDHE Tier 2 risk-based screening level of $5.0 \mu \mathrm{g} / \mathrm{L}$ remain.

In September 2005, the CCC/USDA initiated periodic sampling of groundwater, in accord with a program approved by the KDHE (2005), to monitor carbon tetrachloride concentrations in the groundwater.

Under the KDHE-approved monitoring plan (Argonne 2005b), groundwater is sampled twice yearly and analyzed for VOCs. During the initial 2 yr of monitoring, analysis for selected geochemical parameters was also conducted to aid in the evaluation of possible natural contaminant degradation (reductive dechlorination) processes in the subsurface environment. Consistently low levels of dissolved oxygen (DO) and oxidation-reduction potential (ORP) at monitoring well MW1D (in the deepest portion of the contaminated aquifer) and the presence of chloroform (the primary degradation product of carbon tetrachloride) suggest that some degree of reductive dechlorination is occurring.

The analytical results for groundwater sampling events at Morrill from September 2005 to September 2010 were documented previously (Argonne 2006, 2007a,b, 2008a,b, 2009, 2010, 2011). Those results consistently demonstrated the presence of carbon tetrachloride 
contamination, at concentrations exceeding the KDHE Tier 2 risk-based screening level of $5.0 \mu \mathrm{g} / \mathrm{L}$ for this compound, in a groundwater plume extending southward from the former CCC/USDA facility, toward Terrapin Creek at the southern edge of the town.

Terrapin Creek is identified by the KDHE (2001) as tributary segment 308 to Walnut Creek, which in turn is located in the Big Nemaha Subbasin of the Missouri Basin. Walnut Creek is classified by the KDHE (2001) as impaired because of high levels of fecal coliform bacteria. Prevention of further degradation of Terrapin Creek by carbon tetrachloride is the regulatory driver for ongoing monitoring of the carbon tetrachloride plume (KDHE 2007a). No trend of increasing carbon tetrachloride levels near the creek has been indicated.

In 2006, the CCC/USDA recommended expansion of the approved monitoring program to include the collection and analysis of surface water samples along Terrapin Creek (Argonne 2007a). At the request of the KDHE (2007a), locations for both surface water and shallow sediment sampling were discussed with the KDHE in January 2007. An addendum to the existing monitoring plan (Appendix A in the report of 2009 monitoring [Argonne 2010]) and a standard operating procedure for sediment sampling (SOP AGEM-15; Appendix B in Argonne 2010) were submitted to the KDHE on the basis of these discussions and were subsequently approved (KDHE 2008b). To supplement the original scope of the monitoring, Argonne has also sampled natural vegetation at locations in the contaminant plume and along Terrapin Creek for analyses for VOCs.

In August 2010, indoor air sampling was conducted at seven residences, one church, and one business overlying the contaminant plume to evaluate the potential for vapor intrusion. Carbon tetrachloride contamination was not detected.

The April and October 2011 groundwater sampling events reported here represent a continuation of the approved monitoring program, as requested by the KDHE (2007b). The groundwater sampling is presently conducted, in accord with the monitoring plan (Argonne 2005b) and the addendum to that plan (Appendix A in Argonne 2010), in a network of 12 monitoring wells and 3 private wells at locations approved by the KDHE (2008b). In addition, since 2008, overflow from the Grimm irrigation well (installed in 2008 just south of Terrapin Creek) has also been sampled. 


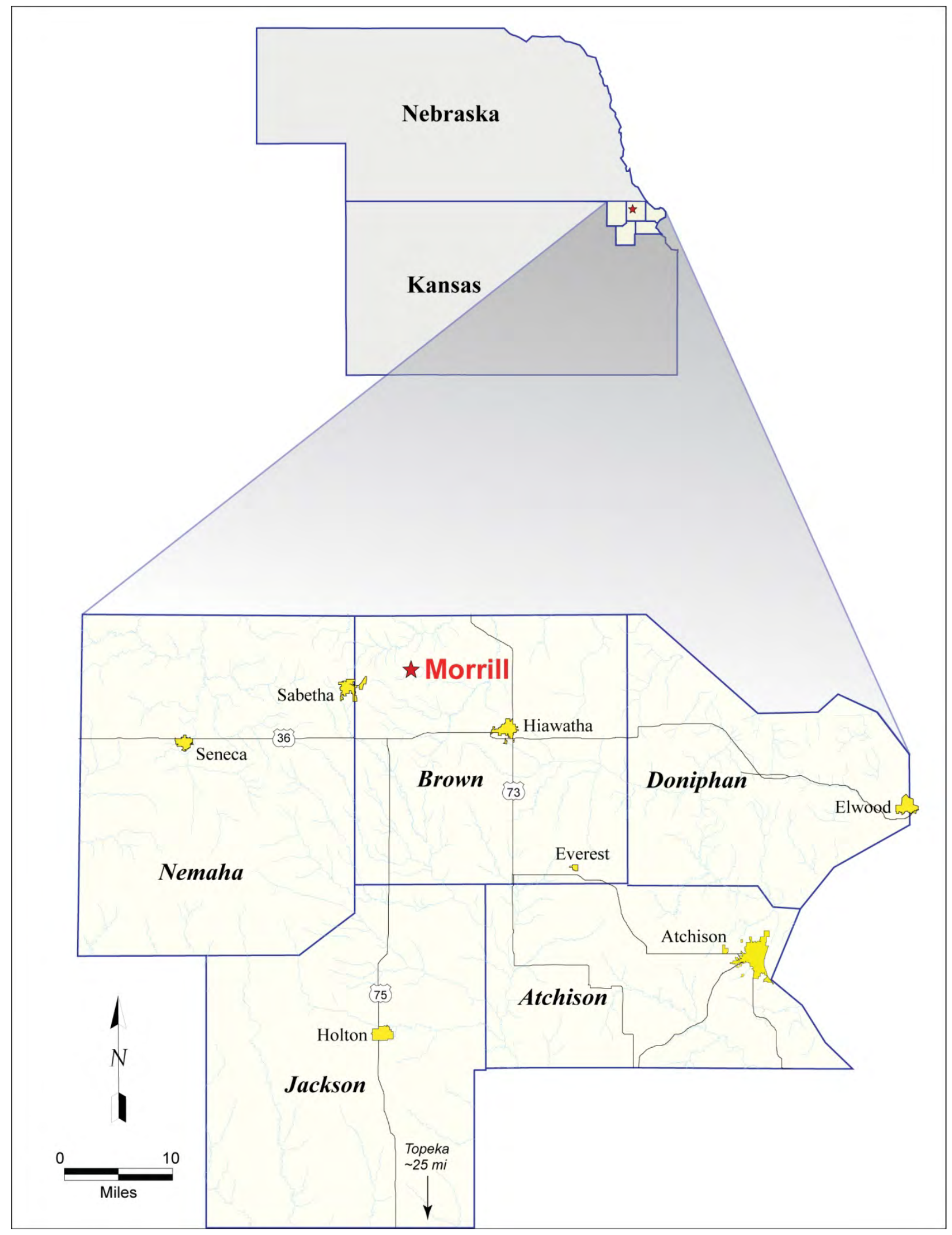

FIGURE 1.1 Location of Brown County and Morrill, Kansas. 


\section{Sample Collection and Analysis Activities}

\subsection{Measurement of Groundwater Levels}

Data recorders currently installed in wells MW1S-MW4S and MW6S-MW8S are gathering long-term data on the groundwater elevation and gradient at Morrill in order to evaluate daily to seasonal variation. In addition, to calibrate the long-term data and to define the potentiometric surface, depths to groundwater and total well depths from the tops of the well casings are measured periodically in conjunction with the data recorder downloads, as well as during each groundwater sampling event, with an accuracy of $\pm 0.01 \mathrm{ft}$.

During the current reporting period, the data recorders were downloaded on April 13, 2011, and January 5, 2012. Water levels were measured manually in all monitoring wells on these dates, as well as during the sampling events on April 20-21, 2011, and October 3-4, 2011.

The groundwater level data are discussed in Section 3.1.

\subsection{Monitoring Well and Private Well Sampling and Analyses}

Monitoring wells MW1D and MW1S-MW11S and the Stone, Isch, and Rilinger private wells (Figure 2.1) were sampled on April 20-21, 2011, and October 3-4, 2011.

Samples were collected from monitoring wells by using a low-flow bladder pump. After measurement of water levels, each monitoring point was purged of a small volume, in accord with U.S. Environmental Protection Agency (EPA) procedure EPA/540/S-95/504 (Puls and Barcelona 1996; Yeskis and Zavala 2002) and the equipment manufacturers' instructions. Field measurements of temperature, $\mathrm{pH}$, conductivity, $\mathrm{DO}$, and ORP were taken during purging until the measurements stabilized. Field measurements of iron(II) were made as outlined in the monitoring plan (Argonne 2005b), in accord with procedures in the Master Work Plan (Argonne 2002). Samples from the Isch and Rilinger private wells were collected after a 5-min purge with the dedicated pump. The sample from the Stone private well was collected after purging of the well by bailing. 
Prior sampling at well MW1S, which is located near the center of the contaminant plume and is screened over a 40 -ft interval, has indicated that a representative sample is not collected by the low-flow sampling procedure. Therefore, since 2008 the well has also been sampled periodically after purging of three well volumes. This comparison sampling is discussed further in Section 3.5.

The sequence of activities during the 2011 sampling events is summarized in Appendix A, Table A.1.

Groundwater samples for VOCs analyses were collected in appropriate laboratory containers, labeled, packaged, and chilled to $4^{\circ} \mathrm{C}$ by placement in ice-filled coolers. The samples were shipped by an overnight delivery service to the Applied Geosciences and Environmental Management (AGEM) Laboratory at Argonne for VOCs analyses with EPA Method 524.2 (EPA 1995). Separate aliquots of selected samples (chosen in the field) were shipped to TestAmerica Laboratories, Inc., South Burlington, Vermont, for verification VOCs analysis.

The groundwater analysis results are presented and discussed in Section 3.2.

\subsection{Surface Water and Sediment Sampling and Analyses}

At the request of the KDHE (2007a), surface water samples and corresponding samples of the underlying shallow sediments in the creek bed are routinely collected for VOCs analyses at five locations along Terrapin Creek (Figure 2.2), as outlined in the monitoring plan addendum (Appendix A in Argonne 2010). The sampling was conducted in accord with procedures in the Master Work Plan (Argonne 2002) and SOP AGEM-15 (Appendix B in Argonne 2010). Surface water flow in Terrapin Creek south of Morrill originates at the outfall from an earthen dam and retention pond approximately 1,900 ft southwest of the former CCC/USDA facility (Figure 2.2). Surface water and sediment sampling location SMB, which is directly downstream from this outfall, is believed to lie upgradient, or cross-gradient, to groundwater flow (and hence possible contaminant migration) from the vicinity of the former CCC/USDA facility. (See Section 3.1.) Sampling locations SM1-SM4 were selected to lie downgradient and downstream from the carbon tetrachloride detections previously identified at MW8S and elsewhere in the monitoring well network. 
Samples of surface water were collected in appropriate containers, labeled, preserved at $4^{\circ} \mathrm{C}$, and shipped by an overnight delivery service to the AGEM Laboratory for VOCs analyses with EPA Method 524.2 (EPA 1995). Samples of the shallow creek bed sediments were collected by scooping the materials directly into appropriate laboratory containers. The samples were labeled, preserved on dry ice, and shipped to the AGEM Laboratory for sample preparation and VOCs analyses with modified EPA Methods 5030B and 8260B.

The surface water and sediment analysis results are presented and discussed in Section 3.3.

\subsection{Vegetation Sampling and Analyses}

Vegetation samples have been collected at locations within the contaminant plume and along Terrapin Creek and its tributaries south and southwest of the former CCC/USDA facility. Vegetation sampling locations, which have expanded over time, were selected along the apparent direction of groundwater flow from the former facility. Vegetation samples were collected at 18 locations in July 2007 (Argonne 2008a), at 25 locations in July 2008 (Argonne 2009), and at 22 locations in August 2009 (Argonne 2010). In July 2010 and July 2011, branch tissue samples were collected at 42 locations from mature ash, cottonwood, elm, hackberry, juniper, maple, mulberry, oak, Osage orange, pear, pine, walnut, and willow trees.

Figure 2.3 illustrates the locations of the vegetation sampling conducted on July 28, 2011. The sequence of sampling activities, including descriptions of sample locations and identifications of the trees sampled, is summarized in Table A.1, Appendix A. Analytical results are presented and discussed in Section 3.4.

The tree tissue samples were collected in appropriate laboratory containers, labeled, preserved on dry ice, and shipped to the AGEM Laboratory for carbon tetrachloride and chloroform analyses by a headspace technique based on a modification of EPA Method 5021 (http://www.epa.gov/epahome/index/; Alvarado and Rose 2004). 


\subsection{Handling and Disposal of Investigation-Derived Waste}

The water generated as potentially contaminated investigation-derived waste was containerized on-site. The accumulated purge water was sampled on October 31, 2011 (along with wastewaters from several other CCC/USDA sites in Kansas), and analyzed by Pace Analytical Services, Inc., Lenexa, Kansas, for VOCs on November 4 with EPA Method 5030/8260, for ethylene dibromide on November 8 with EPA Method 504.1, and for nitrate/nitrite nitrogen on November 2 with EPA Method 353.2. Carbon tetrachloride was detected at $3.4 \mu \mathrm{g} / \mathrm{L}$. Nitrate/nitrite nitrogen was present at $13.8 \mathrm{mg} / \mathrm{L}$. Ethylene dibromide was not detected. The laboratory results are in Supplement 1, on the compact disc (CD) inside the back cover of this report. The water was delivered on December 19, 2011 (together with purge water from several other CCC/USDA investigation sites in Kansas), for disposal at the Sabetha publicly owned wastewater treatment plant.

\subsection{Quality Control for Sample Collection, Handling, and Analysis}

The quality control/quality assurance procedures followed during the 2011 monitoring events are described in detail in the Master Work Plan (Argonne 2002) and SOP AGEM-15 (Appendix B in Argonne 2010). These procedures are summarized as follows:

- Sample collection and handling activities were monitored by the documentation of samples as they were collected and the use of chain-ofcustody forms and custody seals to ensure sample integrity during handling and shipment.

- Samples designated for VOCs analyses were received with custody seals intact and at the appropriate preservation temperature. All samples were analyzed within the required holding times.

- Quality control samples (field blanks, equipment rinsates, and trip blanks) collected to monitor sample collection and handling activities were free of carbon tetrachloride contamination. Method blanks used to monitor analytical methodologies were free of carbon tetrachloride and chloroform contamination. Analytical results for quality control samples collected to monitor sample-handling activities are in Appendix B, Table B.1. 
- Groundwater samples were analyzed for VOCs at the AGEM Laboratory with the purge-and-trap method on a gas chromatograph-mass spectrometer system (modified EPA Method 524.2). Calibration checks with each sample delivery group were required to be within $\pm 20 \%$ of the standard. Surrogate standard determinations performed on samples and blanks were within the specified range of $80-120 \%$ for all samples, in either the initial analysis or a successful reanalysis. Accuracy and precision of the analytical methodology was evident in the analysis of four replicate samples and duplicate analysis of eight additional samples, with an average relative percent difference values of approximately $<5 \%$ between the initial analysis and the associated quality control analysis for both carbon tetrachloride and chloroform (Appendix B, Table B.2). The groundwater analytical data from the AGEM Laboratory are acceptable for quantitative determination of contaminant distribution.

- In accordance with the procedures defined in the Master Work Plan (Argonne 2002), the analyses of water samples at the AGEM Laboratory were verified by a second laboratory. Accordingly, groundwater samples collected during each of the 2011 monitoring events were submitted to TestAmerica for analysis according to the EPA's Contract Laboratory Program methodology. Complete analytical results for three groundwater samples and one trip blank collected in April 2011 are in sample delivery group 200-4827 in Supplement 2 (on CD). Complete analytical results for three groundwater samples, one surface water sample, and one trip blank collected in October 2011 are in sample delivery group 200-7357 in Supplement 2 (on CD). The results are summarized in Appendix B, Table B.3. Although the results from TestAmerica support the results from the AGEM Laboratory, the results for some samples are not comparable. Samples from MW11S with quantitative levels of carbon tetrachloride and from the Rilinger private well with nearquantitative levels compare well between the two laboratories, with relative percent difference values of $<10 \%$. Other samples are not directly comparable between the two laboratories, because trace levels of carbon tetrachloride were detected in blanks associated with the analyses at TestAmerica, including the two trip blanks. 
- Vegetation samples were analyzed for carbon tetrachloride and chloroform at the AGEM Laboratory by using a gas chromatograph with electron capture detection to achieve the low detection limits required. An 11-point calibration of the gas chromatograph system was established on the basis of the mass of known quantities of carbon tetrachloride and chloroform. 


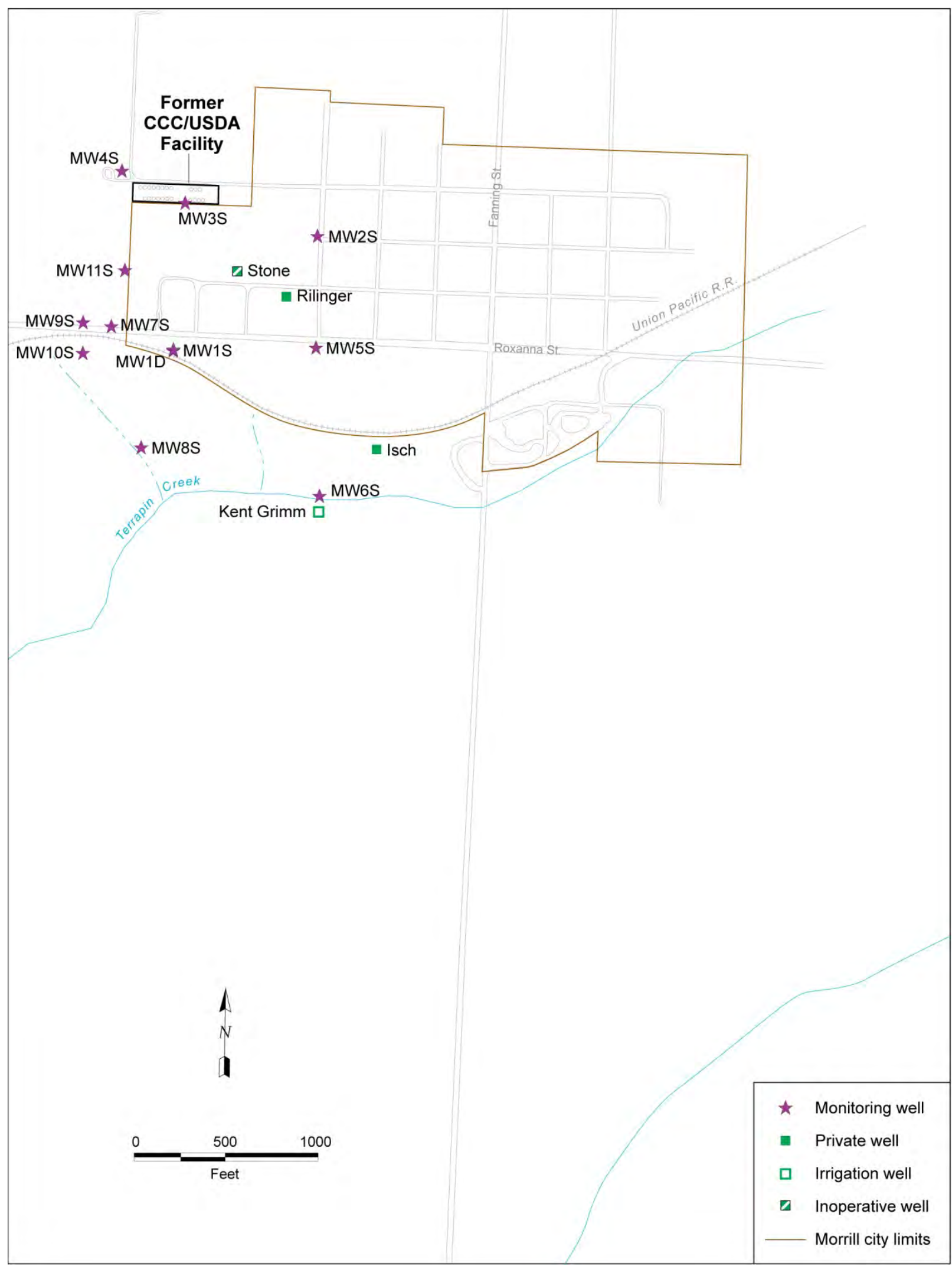

FIGURE 2.1 Groundwater monitoring network as of October 2011. 


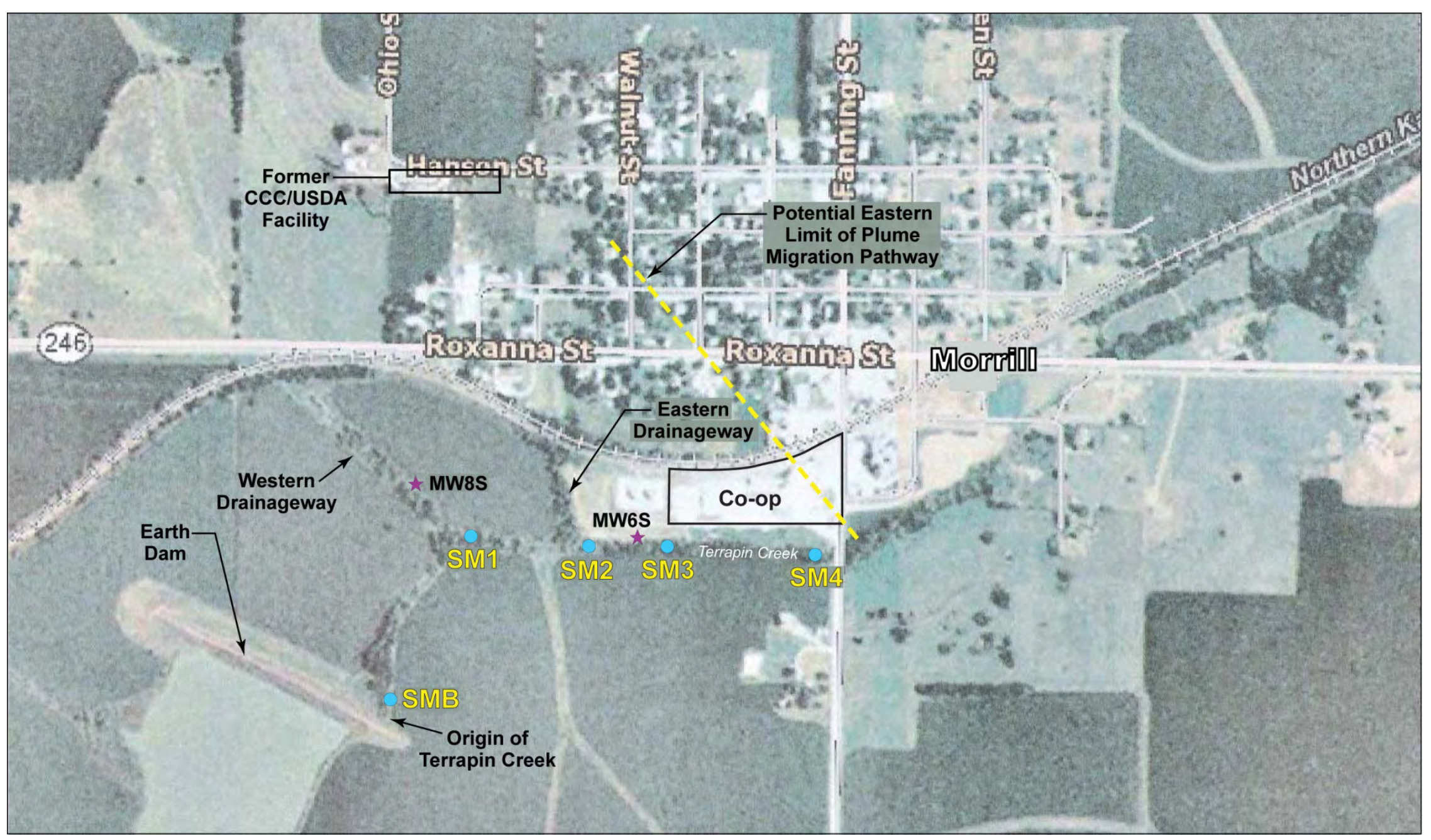

FIGURE 2.2 Locations of surface water and creek bed sediment sampling along Terrapin Creek in October 2011. 


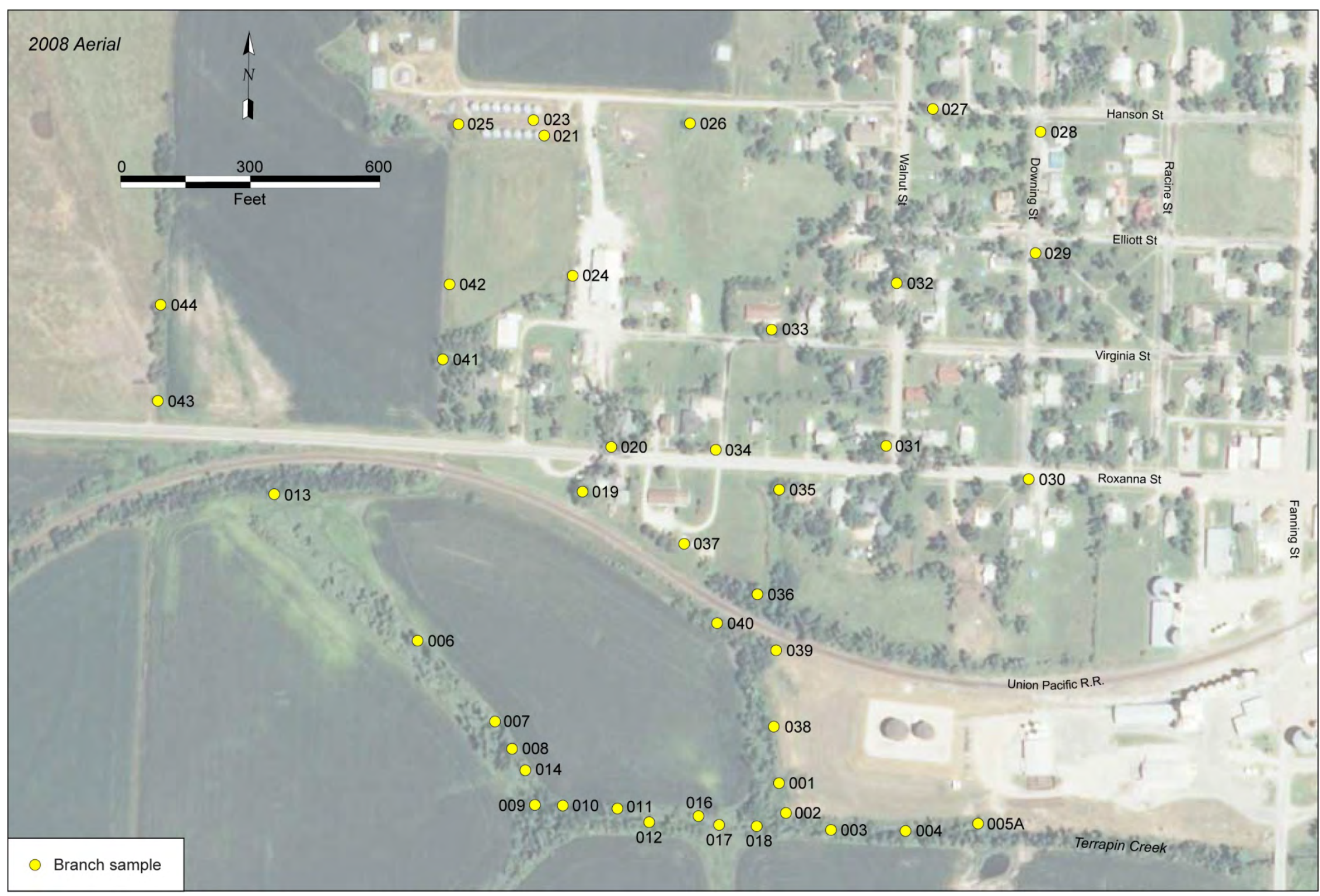

FIGURE 2.3 Locations of native vegetation sampling downgradient of the former CCC/USDA facility and along Terrapin Creek in July 2011. 


\section{Results and Discussion}

\subsection{Groundwater Level Data}

Depths to groundwater were measured manually in all available monitoring wells on April 13, 2011, and January 5, 2012, in conjunction with data recorder downloads. These handmeasured water level data, along with hand-measured levels from the April 20-21, 2011, and October 3-4, 2011, sampling events, are in Table 3.1.

As in previous years, groundwater flow during 2011 (Figure 3.1) was predominantly to the south, from the vicinity of the former CCC/USDA facility toward Terrapin Creek.

Hydrographs recorded in 2011 for the Morrill monitoring wells (Figure 3.2) illustrate rises in water levels reflecting seasonal responses to spring precipitation and recharge, followed by generally declining groundwater levels during the remainder of the year. Similar seasonal responses have been observed annually (to varying extents) throughout Argonne's investigations at Morrill.

The hydrograph in Figure 3.2 for monitoring well MW6S is marked by a series of sharp downward "spikes" in the water level at this location during May and July-August, 2011. The observed events reflect transient drawdown in response to pumping of the Grimm irrigation well (location TD12), which was installed just south of the MW6S location in March 2008 (Argonne 2008b). Little or no distinct response to the pumping of the Grimm irrigation well is apparent at the other monitoring well locations; however, the operation of the Grimm well empirically coincides with the decline in water levels observed at all locations in the summer and fall of 2009-2010. A similar seasonal decline in water levels also observed in summer and fall 2007, in the absence of the Grimm well pumping, suggests that spring precipitation and recharge represent the predominant factors affecting the local groundwater level patterns.

\subsection{Groundwater Analysis Results}

The analytical data for VOCs in the groundwater samples collected in April and October 2011 are in Table 3.2, together with data for the previous sampling events conducted under the KDHE-approved monitoring plan (Argonne 2005b). The results of field measurements on the 
groundwater samples are in Table 3.3. The April and October 2011 data for carbon tetrachloride in groundwater are illustrated in Figures 3.3a and 3.3b, respectively. For comparison, the results of the groundwater sampling in April and September 2010 are in Figures 3.4a and 3.4b, respectively.

In April 2011 (Figure 3.3a), carbon tetrachloride was detected at 9 of the 15 monitoring locations, at concentrations ranging from $<1 \mu \mathrm{g} / \mathrm{L}$ (at the Rilinger private well and 3 monitoring wells) to a maximum of $38 \mu \mathrm{g} / \mathrm{L}$ (at well MW11S). Low levels of chloroform $(<1-1.2 \mu \mathrm{g} / \mathrm{L}$ ) were detected in 5 wells (Table 3.2).

In October 2011 (Figure 3.3b), carbon tetrachloride was detected at 8 of the 15 monitoring locations, at concentrations ranging from $<1 \mu \mathrm{g} / \mathrm{L}$ (at the Rilinger private well and 2 monitoring wells) to a maximum of $49 \mu \mathrm{g} / \mathrm{L}$ (at well MW3S). Low levels of chloroform $(<1-1.8 \mu \mathrm{g} / \mathrm{L})$ were detected in 4 wells (Table 3.2).

The results in Table 3.2, Figures 3.3a,b, and Figures 3.4a,b indicate no significant changes in the concentrations or distribution of carbon tetrachloride in the groundwater during the 2011 review period or in comparison to the results of the spring and fall 2010 monitoring.

\subsection{Surface Water and Sediment Analysis Results}

Table 3.4 presents the results of VOCs analyses of the surface water and shallow sediment samples collected (at the request of the KDHE [2007a]) along Terrapin Creek. No carbon tetrachloride was detected in the surface water samples collected at locations shown in Figure 2.2, at an analytical method detection limit of $0.1 \mu \mathrm{g} / \mathrm{L}$. Similarly, no carbon tetrachloride was identified in the associated sediment samples at an analytical method detection limit of $1.0 \mu \mathrm{g} / \mathrm{kg}$. The 2011 results therefore indicate that the surface waters and underlying sediments of Terrapin Creek remain uncontaminated by carbon tetrachloride.

\subsection{Vegetation Analysis Results and Observations}

The July 2008 vegetation sampling event involved expansion to include locations at and directly downgradient from the former CCC/USDA facility (locations MR019 to MR024; Figure 2.3). The sampling area was expanded further in July 2010 by adding locations MR025- 
MR044 (Figure 2.3) along the pathway from the former facility and toward Terrapin Creek. Essentially the same locations were sampled in July 2011.

Analytical data for carbon tetrachloride and chloroform in tree branch samples collected in July 2011 (and in previous years) are shown in Table 3.5. In the July 2011 sampling, trace concentrations of carbon tetrachloride were identified at 2 of 42 locations sampled (MR037 and MR043; Figure 3.5). The analytical method detection limit was $0.1 \mu \mathrm{g} / \mathrm{kg}$.

\subsection{Comparison of Analytical Results for Samples Collected after Low-Flow Purging and after Purging of Three Well Volumes}

Of particular concern at Morrill has been the applicability of the low-flow sampling method for the wells installed by the KDHE in 1995 with screen intervals of 30-40 ft. At the request of the KDHE (2008a), selected wells were sampled in October 2008 by using both the low-flow purging technique and the three-well-volume purging technique to confirm the suitability of the low-flow method for groundwater sampling at Morrill. As reported previously (Argonne 2009), samples were collected by both methods in October 2008 from wells MW1S, MW2S, and MW3S (installed by the KDHE in 1995 with 30- to 40-ft screens), as well as from well MW11S (installed by Argonne in 2004 with a 15-ft screen). Results for all pairs except the MW1S samples compared favorably; the low-flow results for well MW1S (centrally located in the plume) were dramatically lower than the three-volume-purge results. Low-flow results for MW1S in October 2008 were therefore considered non-representative, and the value for the sample obtained after purging of three well volumes was honored instead (Argonne 2009). Sampling conducted in later years has continued this evaluation of the suitability of the low-flow sampling method.

In October 2011, sampling of well MW1S with the low-flow procedure resulted in a carbon tetrachloride detection at $1.7 \mu \mathrm{g} / \mathrm{L}$. Subsequent sampling with the three-volume-purge method resulted in a carbon tetrachloride detection of $23 \mu \mathrm{g} / \mathrm{L}$. This higher concentration is considered representative of the contaminant level at this location. This was the fourth sampling event (October 2008, September 2009, April 2010, and October 2011) in which a similar pattern was observed for well MW1S. 
TABLE 3.1 Hand-measured groundwater levels in 2011.

\begin{tabular}{|c|c|c|c|c|c|c|c|c|c|}
\hline \multirow[b]{2}{*}{ Well } & \multirow[b]{2}{*}{$\begin{array}{l}\text { Top of Casing } \\
\text { Elevation } \\
\text { (ft AMSL) }\end{array}$} & \multicolumn{2}{|c|}{ April 13, 2011} & \multicolumn{2}{|c|}{ April 20-21, 2011} & \multicolumn{2}{|c|}{ October 3-4, 2011} & \multicolumn{2}{|c|}{ January 5, 2012} \\
\hline & & $\begin{array}{l}\text { Depth to } \\
\text { Water } \\
\text { (ft BGL) }\end{array}$ & $\begin{array}{l}\text { Groundwater } \\
\text { Elevation } \\
\text { (ft AMSL) }\end{array}$ & $\begin{array}{l}\text { Depth to } \\
\text { Water } \\
\text { (ft BGL) }\end{array}$ & $\begin{array}{l}\text { Groundwater } \\
\text { Elevation } \\
\text { (ft AMSL) }\end{array}$ & $\begin{array}{l}\text { Depth to } \\
\text { Water } \\
\text { (ft BGL) }\end{array}$ & $\begin{array}{l}\text { Groundwater } \\
\text { Elevation } \\
\text { (ft AMSL) }\end{array}$ & $\begin{array}{l}\text { Depth to } \\
\text { Water } \\
\text { (ft BGL) }\end{array}$ & $\begin{array}{c}\text { Groundwater } \\
\text { Elevation } \\
\text { (ft AMSL) }\end{array}$ \\
\hline MW1S & 1124.68 & 27.40 & 1097.28 & 23.45 & 1101.23 & 21.80 & 1102.88 & 24.61 & 1100.07 \\
\hline MW1D & 1124.63 & 32.51 & 1092.12 & 30.15 & 1094.48 & 29.06 & 1095.57 & 31.90 & 1092.73 \\
\hline MW2S & 1137.07 & 37.39 & 1099.68 & 36.00 & 1101.07 & 32.73 & 1104.34 & 33.25 & 1103.82 \\
\hline MW3S & 1135.76 & 32.76 & 1103.00 & 32.06 & 1103.70 & 25.95 & 1109.81 & 25.95 & 1109.81 \\
\hline MW4S & 1143.61 & 42.18 & 1101.43 & 42.39 & 1101.22 & 35.60 & 1108.01 & 36.48 & 1107.13 \\
\hline MW5S & 1122.21 & 26.19 & 1096.02 & 24.43 & 1097.78 & 21.10 & 1101.11 & 22.38 & 1099.83 \\
\hline MW6S & 1090.97 & 6.16 & 1084.81 & 6.52 & 1084.45 & 6.49 & 1084.48 & 6.03 & 1084.94 \\
\hline MW7S & 1119.86 & 20.64 & 1099.22 & 19.69 & 1100.17 & 7.11 & 1112.75 & 16.86 & 1103.00 \\
\hline MW8S & 1098.53 & 3.84 & 1094.69 & 2.73 & 1095.80 & 4.91 & 1093.62 & 2.57 & 1095.96 \\
\hline MW9S & 1118.31 & 23.96 & 1094.35 & 23.76 & 1094.55 & 22.35 & 1095.96 & 22.76 & 1095.55 \\
\hline MW10S & 1110.78 & 14.17 & 1096.61 & 13.69 & 1097.09 & 12.54 & 1098.24 & 12.48 & 1098.30 \\
\hline MW11S & 1133.08 & 39.59 & 1093.49 & 39.16 & 1093.92 & 36.83 & 1096.25 & 36.88 & 1096.20 \\
\hline
\end{tabular}


TABLE 3.2 Results of analyses at the AGEM Laboratory for volatile organic compounds in groundwater samples collected in 2003-2011. Shading indicates sample collection with the low-flow procedure.

\begin{tabular}{|c|c|c|c|c|c|c|c|c|c|c|c|c|}
\hline \multirow[b]{2}{*}{ Location } & \multirow[b]{2}{*}{$\begin{array}{l}\text { Screen } \\
\text { Interval } \\
\text { (ft BGL) }\end{array}$} & \multirow[b]{2}{*}{$\begin{array}{l}\text { Sample } \\
\text { Date }\end{array}$} & \multirow[b]{2}{*}{$\begin{array}{l}\text { Depth to } \\
\text { Water } \\
\text { (ft BOTC) }\end{array}$} & \multirow[b]{2}{*}{$\begin{array}{l}\text { Depth of } \\
\text { well } \\
\text { (ft BTOC) }\end{array}$} & \multirow[b]{2}{*}{$\begin{array}{l}\text { Volume } \\
\text { Purged }\end{array}$} & \multirow[b]{2}{*}{$\begin{array}{l}\text { Purge } \\
\text { Units }\end{array}$} & \multirow{2}{*}{$\begin{array}{l}\text { Pump } \\
\text { Intake } \\
\text { Position } \\
\text { (ft BGL) }\end{array}$} & \multicolumn{3}{|c|}{ Concentration $(\mu \mathrm{g} / \mathrm{L})$} & \multirow[b]{2}{*}{ Comment } & \multirow[b]{2}{*}{ Sample } \\
\hline & & & & & & & & $\begin{array}{c}\text { Carbon } \\
\text { Tetrachloride }\end{array}$ & Chloroform & $\begin{array}{l}\text { Methylene } \\
\text { Chloride }\end{array}$ & & \\
\hline MW1S & $11-51$ & $10 / 23 / 03$ & 30.4 & 54.0 & 70 & gal & - & 33 & 1.6 & $N D^{a}$ & & MRMW1S-W-16422 \\
\hline MW1S & $11-51$ & $6 / 2 / 04$ & 27.0 & 53.9 & 53 & gal & - & 19 & 0.9 & ND & & MRMW1S-W-16461 \\
\hline MW1S & $11-51$ & $9 / 13 / 05$ & 24.2 & 53.9 & 57 & gal & - & 35 & 1.7 & ND & & MRMW1S-W-19259 \\
\hline MW1S & $11-51$ & $3 / 22 / 06$ & 29.0 & 54.0 & 48 & gal & - & 40 & 1.8 & ND & & MRMW1S-W-20008 \\
\hline MW1S & $11-51$ & 9/20/06 & 26.8 & 54.0 & 55 & gal & - & 23 & $0.9 \mathrm{~J}^{\mathrm{b}}$ & ND & & MRMW1S-W-22495 \\
\hline MW1S & $11-51$ & $3 / 21 / 07$ & 25.8 & 54.0 & 55 & gal & - & 23 & 1.1 & ND & & MRMW1S-W-16488 \\
\hline MW1S & $11-51$ & 10/1/07 & 21.7 & 54.0 & 63 & gal & - & 56 & 2.7 & ND & & MRMW1S-W-16595 \\
\hline MW1S & $11-51$ & $4 / 14 / 08$ & 16.2 & 54.0 & 5.5 & $\mathrm{~L}$ & - & $0.3 \mathrm{~J}$ & ND & ND & April monitoring. & MRMW1S-W-23230 \\
\hline MW1S & $11-51$ & $4 / 22 / 08$ & 16.0 & 54.0 & 6.3 & $\mathrm{~L}$ & - & $0.2 \mathrm{~J}$ & ND & ND & $\begin{array}{l}\text { Confirm low carbon } \\
\text { tetrachloride. }\end{array}$ & MRMW1S-W-23259 \\
\hline MW1S & $11-51$ & $5 / 1 / 08$ & - & - & 3.2 & L & 22.0 & ND & ND & ND & Top of screen. & MRMW1S-22-W-23275 \\
\hline MW1S & $11-51$ & $5 / 1 / 08$ & - & - & 3.2 & L & 27.0 & ND & ND & ND & Middle of screen. & MRMW1S-27-W-23276 \\
\hline MW1S & $11-51$ & $5 / 1 / 08$ & - & - & 4.3 & L & 48.0 & $0.3 \mathrm{~J}$ & ND & ND & Bottom of screen. & MRMW1S-48-W-23277 \\
\hline MW1S & $11-51$ & $10 / 20 / 08$ & 25.8 & 54.0 & 6.0 & L & 31.0 & $0.7 \mathrm{~J}$ & ND & ND & Low flow. & MRMW1S-W-27620 \\
\hline MW1S & $11-51$ & $10 / 21 / 08$ & - & - & - & - & - & 35 & 1.8 & ND & Full purge. & MRMW1S-W-27649 \\
\hline MW1S & $11-51$ & $4 / 24 / 09$ & 24.4 & 54.0 & 5.0 & L & 39.2 & ND & ND & ND & & MRMW1S-W-27652 \\
\hline MW1S & $11-51$ & $9 / 3 / 09$ & 19.0 & 54.0 & 8.0 & L & 35.0 & ND & ND & ND & Low flow. & MRMW1S-W-29942 \\
\hline MW1S & $11-51$ & $9 / 4 / 09$ & 19.3 & 51.2 & 244 & L & 50.0 & 34 & 1.7 & ND & Three well volumes. & MRMW1S-W-29971 \\
\hline MW1S & $11-51$ & $4 / 7 / 10$ & 11.7 & 51.3 & 7.0 & $\mathrm{~L}$ & 16.6 & ND & ND & ND & Top of screen. & MRMW1ST-W-29981 \\
\hline MW1S & $11-51$ & $4 / 7 / 10$ & 11.6 & 51.3 & 6.0 & L & 31.5 & ND & ND & ND & Middle of screen. & MRMW1SM-W-29980 \\
\hline MW1S & $11-51$ & $4 / 7 / 10$ & 11.7 & 51.3 & 6.0 & L & 46.3 & ND & ND & ND & Bottom of screen. & MRMW1SB-W-29979 \\
\hline MW1S & $11-51$ & $4 / 7 / 10$ & 11.5 & 51.3 & 80 & gal & 49.0 & 21 & 1.2 & ND & Three well volumes. & MRMW1S3X-W-29982 \\
\hline MW1S & $11-51$ & $9 / 22 / 10$ & 19.9 & 54.0 & 10 & L & 31.0 & 1.6 & ND & ND & Low flow. & MRMW1S-W-30010 \\
\hline MW1S & $11-51$ & $4 / 20 / 11$ & 23.5 & 54.0 & 6.5 & L & 37.0 & $0.3 \mathrm{~J}$ & ND & ND & Low flow. & MRMW1S-W-30038 \\
\hline MW1S & $11-51$ & $10 / 4 / 11$ & 21.8 & 54.0 & 8.0 & L & 34.9 & 1.7 & ND & ND & Low flow. & MRMW1S-W-30067 \\
\hline MW1S & $11-51$ & $10 / 12 / 11$ & 22.1 & 54.0 & 6.0 & L & 36.6 & 1.5 & ND & ND & Low flow. & MRMW1S-W-30091 \\
\hline MW1S & $11-51$ & $10 / 12 / 11$ & 22.4 & 54.0 & 65 & gal & 49.0 & 23 & 1.2 & ND & Three well volumes. & MRMW1S3X-W-30093 \\
\hline MW1D & $63-88$ & $10 / 22 / 03$ & 28.4 & 88.5 & 92 & gal & - & ND & ND & ND & & MRMW1D-W-16421 \\
\hline MW1D & $63-88$ & $6 / 2 / 04$ & 26.8 & 88.6 & 140 & gal & - & ND & ND & ND & & MRMW1D-W-16458 \\
\hline MW1D & $63-88$ & $9 / 13 / 05$ & 23.7 & 88.6 & 200 & gal & - & ND & ND & ND & & MRMW1D-W-16518 \\
\hline MW1D & $63-88$ & 3/19/06 & 26.9 & 88.6 & 112 & gal & - & ND & ND & $0.4 \mathrm{~J} \mathrm{~B}^{\mathrm{c}}$ & & MRMW1D-W-19986 \\
\hline MW1D & $63-88$ & 9/20/06 & 25.5 & 88.8 & 125 & gal & - & ND & ND & ND & & MRMW1D-W-16532 \\
\hline MW1D & $63-88$ & $3 / 21 / 07$ & 25.8 & 88.8 & 125 & gal & - & ND & ND & ND & & MRMW1D-W-16487 \\
\hline MW1D & $63-88$ & $10 / 1 / 07$ & 22.8 & 89.4 & 130 & gal & - & ND & ND & ND & & MRMW1D-W-16596 \\
\hline MW1D & $63-88$ & $4 / 14 / 08$ & 29.5 & 89.0 & 6.0 & L & - & ND & ND & ND & & MRMW1D-W-23231 \\
\hline MW1D & $63-88$ & $10 / 20 / 08$ & 30.4 & 89.0 & 7.0 & L & 75.5 & ND & ND & ND & & MRMW1D-W-27621 \\
\hline MW1D & $63-88$ & $4 / 24 / 09$ & 31.0 & 89.0 & 7.0 & L & 75.5 & ND & ND & ND & & MRMW1D-W-27653 \\
\hline MW1D & $63-88$ & $9 / 3 / 09$ & 27.1 & 89.0 & 6.5 & L & 75.5 & ND & ND & ND & & MRMW1D-W-29943 \\
\hline MW1D & $63-88$ & $4 / 6 / 10$ & 24.7 & 89.0 & 8.5 & L & 75.5 & ND & ND & ND & & MRMW1D-W-29983 \\
\hline MW1D & $63-88$ & 9/22/10 & 27.9 & 89.0 & 9.0 & L & 75.5 & ND & ND & ND & & MRMW1D-W-30011 \\
\hline MW1D & $63-88$ & $4 / 20 / 11$ & 30.2 & 89.0 & 5.5 & $\bar{L}$ & 75.5 & ND & ND & ND & & MRMW1D-W-30039 \\
\hline MW1D & $63-88$ & $10 / 4 / 11$ & 29.1 & 89.0 & 6.5 & L & 75.5 & ND & ND & ND & & MRMW1D-W-30068 \\
\hline MW1D & $63-88$ & $10 / 4 / 11$ & 29.1 & 89.0 & 6.5 & L & 75.5 & ND & ND & ND & Replicate. & MRMW1DDUP-W-30084 \\
\hline
\end{tabular}


TABLE 3.2 (Cont.)

\begin{tabular}{|c|c|c|c|c|c|c|c|c|c|c|c|c|}
\hline \multirow[b]{2}{*}{ Location } & \multirow{2}{*}{$\begin{array}{l}\text { Screen } \\
\text { Interval } \\
\text { (ft BGL) }\end{array}$} & \multirow[b]{2}{*}{$\begin{array}{l}\text { Sample } \\
\text { Date }\end{array}$} & \multirow{2}{*}{$\begin{array}{l}\text { Depth to } \\
\text { Water } \\
\text { (ft BOTC) }\end{array}$} & \multirow{2}{*}{$\begin{array}{l}\text { Depth of } \\
\text { well } \\
\text { (ft BTOC) }\end{array}$} & \multirow[b]{2}{*}{$\begin{array}{l}\text { Volume } \\
\text { Purged }\end{array}$} & \multirow[b]{2}{*}{$\begin{array}{l}\text { Purge } \\
\text { Units }\end{array}$} & \multirow{2}{*}{$\begin{array}{l}\text { Pump } \\
\text { Intake } \\
\text { Position } \\
\text { (ft BGL) }\end{array}$} & \multicolumn{3}{|c|}{ Concentration $(\mu \mathrm{g} / \mathrm{L})$} & \multirow[b]{2}{*}{ Comment } & \multirow[b]{2}{*}{ Sample } \\
\hline & & & & & & & & $\begin{array}{l}\text { Carbon } \\
\text { Tetrachloride }\end{array}$ & Chloroform & $\begin{array}{l}\text { Methylene } \\
\text { Chloride }\end{array}$ & & \\
\hline MW2S & $13-53$ & $10 / 22 / 03$ & 42.2 & 53.4 & \multicolumn{3}{|c|}{ Purged dry, sampled. } & ND & ND & ND & & MRMW02-W-16419 \\
\hline MW2S & $13-53$ & $6 / 2 / 04$ & 37.4 & 53.3 & 31 & gal & - & ND & ND & ND & & MRMW2S-W-16459 \\
\hline MW2S & $13-53$ & 9/14/05 & 33.7 & 53.3 & 38 & gal & - & ND & ND & ND & & MRMW2S-W-19264 \\
\hline MW2S & $13-53$ & $3 / 21 / 06$ & 40.9 & 53.3 & 27 & gal & - & ND & ND & ND & & MRMW2S-W-19992 \\
\hline MW2S & $13-53$ & 9/18/06 & 36.5 & 53.3 & 28 & gal & - & ND & ND & ND & & MRMW2S-W-22488 \\
\hline MW2S & $13-53$ & $3 / 22 / 07$ & 35.8 & 53.3 & 35 & gal & - & ND & ND & ND & & MRMW2S-W-16559 \\
\hline MW2S & $13-53$ & $10 / 3 / 07$ & 31.2 & 53.4 & 44 & gal & - & ND & ND & ND & & MRMW2S-W-16587 \\
\hline MW2S & $13-53$ & $4 / 15 / 08$ & 23.6 & 53.4 & 2.2 & $\mathrm{~L}$ & - & ND & ND & ND & & MRMW2S-W-23232 \\
\hline MW2S & $13-53$ & $10 / 21 / 08$ & 33.7 & 53.5 & 5.0 & L & 33.0 & ND & ND & ND & Low flow. & MRMW2S-W-27622 \\
\hline MW2S & $13-53$ & 10/21/08 & - & - & - & - & - & ND & ND & ND & Full purge. & MRMW2S-W-27652 \\
\hline MW2S & $13-53$ & $4 / 23 / 09$ & 33.2 & 53.5 & 6.5 & L & 43.4 & ND & $0.6 \mathrm{~J}$ & ND & \multirow{7}{*}{ Replicate. } & MRMW2S-W-27654 \\
\hline MW2S & $13-53$ & 9/3/09 & 29.4 & 53.5 & 5.4 & $\mathrm{~L}$ & 41.2 & ND & ND & ND & & MRMW2S-W-29944 \\
\hline MW2S & $13-53$ & $4 / 6 / 10$ & 18.6 & 52.4 & 6.5 & $\mathrm{~L}$ & 36.0 & ND & ND & ND & & MRMW2S-W-29984 \\
\hline MW2S & $13-53$ & $9 / 22 / 10$ & 31.3 & 53.0 & 7.0 & $\mathrm{~L}$ & 33.0 & ND & ND & ND & & MRMW2S-W-30012 \\
\hline MW2S & $13-53$ & $4 / 20 / 11$ & 36.0 & 53.5 & 8.0 & L & 44.5 & ND & ND & ND & & MRMW2S-W-30040 \\
\hline MW2S & $13-53$ & $4 / 20 / 11$ & 36.0 & 53.5 & 8.0 & $\mathrm{~L}$ & 44.5 & ND & ND & ND & & MRMW2SDUP-W-30055 \\
\hline MW2S & $13-53$ & $10 / 4 / 11$ & 32.7 & 53.5 & 7.5 & $\bar{L}$ & 42.6 & ND & ND & ND & & MRMW2S-W-30069 \\
\hline MW3S & $18-48$ & $10 / 23 / 03$ & 36.5 & 47.8 & 73 & gal & - & 89 & 2.7 & ND & & MRMW03-W-16423 \\
\hline MW3S & $18-48$ & $6 / 2 / 04$ & 30.7 & 47.5 & 34 & gal & - & 110 & 3.2 & ND & & MRMW3S-W-16462 \\
\hline MW3S & $18-48$ & 9/13/05 & 25.6 & 47.6 & 50 & gal & - & 101 & 3.2 & ND & & MRMW3S-W-19261 \\
\hline MW3S & $18-48$ & $3 / 23 / 06$ & 35.6 & 47.7 & 28 & gal & - & 91 & 2.6 & ND & & MRMW3S-W-19994 \\
\hline MW3S & $18-48$ & 9/20/06 & 29.4 & 47.8 & 22 & gal & - & 49 & 1.5 & ND & & MRMW3S-W-22496 \\
\hline MW3S & $18-48$ & $3 / 22 / 07$ & 26.2 & 47.8 & 45 & gal & - & 84 & 2.3 & ND & & MRMW3S-W-16563 \\
\hline MW3S & $18-48$ & $10 / 3 / 07$ & 22.7 & 47.9 & 50 & gal & - & 61 & 2.0 & ND & & MRMW3S-W-16585 \\
\hline MW3S & $18-48$ & $4 / 14 / 08$ & 17.0 & 47.8 & 3.3 & $\mathrm{~L}$ & - & 8.2 & $0.4 \mathrm{~J}$ & ND & April monitoring. & MRMW3S-W-23233 \\
\hline MW3S & $18-48$ & 4/22/08 & 15.8 & 47.8 & 6.5 & $\mathrm{~L}$ & - & $0.7 \mathrm{~J}$ & ND & ND & $\begin{array}{l}\text { Confirm low carbon } \\
\text { tetrachloride. }\end{array}$ & MRMW3S-W-23260 \\
\hline MW3S & $18-48$ & $5 / 1 / 08$ & - & - & 2.6 & L & 26.0 & $0.4 \mathrm{~J}$ & ND & ND & Top of screen. & MRMW3S-26-W-23269 \\
\hline MW3S & $18-48$ & $5 / 1 / 08$ & - & - & 2.8 & $\mathrm{~L}$ & 38.0 & $0.4 \mathrm{~J}$ & ND & ND & Middle of screen. & MRMW3S-38-W-23270 \\
\hline MW3S & $18-48$ & $5 / 1 / 08$ & - & - & 3.2 & $\mathrm{~L}$ & 45.0 & $0.5 \mathrm{~J}$ & ND & ND & Bottom of screen. & MRMW3S-45-W-23271 \\
\hline MW3S & $18-48$ & $10 / 21 / 08$ & 27.0 & 47.8 & 4.2 & L & 33.0 & 55 & 1.4 & ND & Low flow. & MRMW3S-W-27623 \\
\hline MW3S & $18-48$ & $10 / 21 / 08$ & - & - & - & - & - & 63 & 1.6 & ND & Full purge. & MRMW3S-W-27650 \\
\hline MW3S & $18-48$ & $4 / 23 / 09$ & 26.7 & 47.8 & 5.0 & $\mathrm{~L}$ & 37.3 & 29 & 1.4 & ND & & MRMW3S-W-27655 \\
\hline MW3S & $18-48$ & 9/3/09 & 22.4 & 47.8 & 5.5 & L & 35.2 & 30 & 1.1 & ND & Low flow. & MRMW3S-W-29945 \\
\hline MW3S & $18-48$ & $9 / 4 / 09$ & 22.6 & 47.8 & 190 & $\mathrm{~L}$ & 46.0 & 28 & $0.9 \mathrm{~J}$ & ND & Three well volumes. & MRMW3S-W-29972 \\
\hline MW3S & $18-48$ & $4 / 6 / 10$ & 12.1 & 47.8 & 6.0 & $\mathrm{~L}$ & 33.0 & 3.5 & $0.2 \mathrm{~J}$ & ND & & MRMW3S-W-29985 \\
\hline MW3S & $18-48$ & $9 / 23 / 10$ & 23.7 & 47.8 & 7.0 & $\bar{L}$ & 33.0 & 47 & 1.6 & ND & & MRMW3S-W-30013 \\
\hline MW3S & $18-48$ & $4 / 20 / 11$ & 32.1 & 47.8 & 6.2 & $\mathrm{~L}$ & 40.0 & 33 & 1.2 & ND & & MRMW3S-W-30041 \\
\hline MW3S & $18-48$ & $10 / 4 / 11$ & 26.0 & 47.8 & 7.0 & $\bar{L}$ & 37.0 & 49 & 1.8 & ND & & MRMW3S-W-30070 \\
\hline MW4S & $17-47$ & $10 / 21 / 03$ & 46.4 & 47.8 & Purg & dry, sa & npled. & ND & ND & ND & & MRMW04-W-16418 \\
\hline MW4S & $17-47$ & $6 / 4 / 04$ & 43.2 & 47.8 & 10 & gal & - & ND & ND & ND & & MRMW4S-W-16470 \\
\hline MW4S & $17-47$ & 9/14/05 & 36.2 & 47.8 & 8.0 & gal & - & ND & ND & ND & & MRMW4S-W-19262 \\
\hline MW4S & $17-47$ & $3 / 21 / 06$ & 44.6 & 47.7 & 6.0 & gal & - & ND & ND & ND & & MRMW4S-W-19993 \\
\hline MW4S & $17-47$ & 9/18/06 & 41.6 & 47.8 & 5.3 & gal & - & ND & ND & ND & & MRMW4S-W-22487 \\
\hline MW4S & $17-47$ & $3 / 22 / 07$ & 38.7 & 47.8 & 6.0 & gal & - & ND & ND & ND & & MRMW4S-W-16562 \\
\hline MW4S & $17-47$ & $10 / 3 / 07$ & 31.1 & 47.7 & 30 & gal & - & $0.5 \mathrm{JR}^{\mathrm{d}}$ & ND & ND & & MRMW4S-W-16586 \\
\hline
\end{tabular}


TABLE 3.2 (Cont.)

\begin{tabular}{|c|c|c|c|c|c|c|c|c|c|c|c|c|}
\hline \multirow[b]{2}{*}{ Location } & \multirow{2}{*}{$\begin{array}{l}\text { Screen } \\
\text { Interval } \\
\text { (ft BGL) }\end{array}$} & \multirow[b]{2}{*}{$\begin{array}{l}\text { Sample } \\
\text { Date }\end{array}$} & \multirow{2}{*}{$\begin{array}{l}\text { Depth to } \\
\text { Water } \\
\text { (ft BOTC) }\end{array}$} & \multirow{2}{*}{$\begin{array}{l}\text { Depth of } \\
\text { well } \\
\text { (ft BTOC) }\end{array}$} & \multirow[b]{2}{*}{$\begin{array}{l}\text { Volume } \\
\text { Purged }\end{array}$} & \multirow[b]{2}{*}{$\begin{array}{l}\text { Purge } \\
\text { Units }\end{array}$} & \multirow{2}{*}{$\begin{array}{l}\text { Pump } \\
\text { Intake } \\
\text { Position } \\
\text { (ft BGL) }\end{array}$} & \multicolumn{3}{|c|}{ Concentration $(\mu \mathrm{g} / \mathrm{L})$} & \multirow[b]{2}{*}{ Comment } & \multirow[b]{2}{*}{ Sample } \\
\hline & & & & & & & & $\begin{array}{l}\text { Carbon } \\
\text { Tetrachloride }\end{array}$ & Chloroform & $\begin{array}{l}\text { Methylene } \\
\text { Chloride }\end{array}$ & & \\
\hline MW4S & $17-47$ & $1 / 11 / 08$ & - & - & - & - & - & ND & ND & ND & & MOMW4S-W-011108 \\
\hline MW4S & $17-47$ & $4 / 14 / 08$ & 26.3 & 47.9 & 2.5 & L & - & ND & ND & ND & & MRMW4S-W-23234 \\
\hline MW4S & $17-47$ & $10 / 20 / 08$ & 36.7 & 47.8 & 8.0 & gal & - & ND & ND & ND & & MRMW4S-W-27624 \\
\hline MW4S & $17-47$ & $4 / 23 / 09$ & 41.5 & 47.8 & 5.0 & L & 44.7 & ND & ND & ND & & MRMW4S-W-27656 \\
\hline MW4S & $17-47$ & $9 / 4 / 09$ & 31.6 & 47.8 & 6.0 & L & 39.3 & ND & ND & ND & & MRMW4S-W-29946 \\
\hline MW4S & $17-47$ & $4 / 6 / 10$ & 21.8 & 47.9 & 5.5 & L & 34.8 & ND & ND & ND & & MRMW4S-W-29986 \\
\hline MW4S & $17-47$ & $9 / 22 / 10$ & 33.2 & 47.8 & 6.0 & L & 32.0 & ND & ND & ND & & MRMW4S-W-30014 \\
\hline MW4S & $17-47$ & $4 / 21 / 11$ & 42.4 & 47.8 & 5.0 & L & 44.7 & ND & ND & ND & & MRMW4S-W-30042 \\
\hline MW4S & $17-47$ & $10 / 4 / 11$ & 35.6 & 47.8 & 6.0 & L & 40.9 & ND & ND & ND & & MRMW4S-W-30071 \\
\hline MW5S & $15-55$ & $10 / 22 / 03$ & 31.4 & 55.7 & 48 & gal & - & 5.8 & ND & ND & & MRMW05-W-16420 \\
\hline MW5S & $15-55$ & $6 / 2 / 04$ & 26.3 & 55.7 & $>57$ & gal & - & 7.0 & ND & ND & & MRMW5S-W-16460 \\
\hline MW5S & $15-55$ & $9 / 13 / 05$ & 22.7 & 54.2 & 75 & gal & - & 6.3 & $0.2 \mathrm{~J}$ & ND & & MRMW5S-W-19260 \\
\hline MW5S & $15-55$ & $3 / 22 / 06$ & 28.6 & 54.5 & 50 & gal & - & 7.3 & $0.2 \mathrm{~J}$ & ND & & MRMW5S-W-19996 \\
\hline MW5S & $15-55$ & $9 / 20 / 06$ & 25.4 & 54.6 & 52 & gal & - & 6.4 & $0.3 \mathrm{~J}$ & ND & & MRMW5S-W-22493 \\
\hline MW5S & $15-55$ & $3 / 22 / 07$ & 25.1 & 54.6 & 58 & gal & - & 6.5 & $0.4 \mathrm{~J}$ & ND & & MRMW5S-W-16569 \\
\hline MW5S & $15-55$ & $10 / 3 / 07$ & 19.6 & 54.7 & 68 & gal & - & 4.0 & $0.3 \mathrm{~J}$ & ND & & MRMW5S-W-16588 \\
\hline MW5S & $15-55$ & $4 / 14 / 08$ & 11.2 & 54.6 & 6.0 & L & - & ND & ND & ND & April monitoring. & MRMW5S-W-23235 \\
\hline MW5S & $15-55$ & $4 / 23 / 08$ & 11.3 & 54.6 & 6.5 & $\mathrm{~L}$ & - & ND & ND & ND & $\begin{array}{l}\text { Confirm low carbon } \\
\text { tetrachloride. }\end{array}$ & MRMW5S-W-23266 \\
\hline MW5S & $15-55$ & $5 / 1 / 08$ & - & - & 3.7 & L & 20.0 & ND & ND & ND & Top of screen. & MRMW5S-20-W-23272 \\
\hline MW5S & $15-55$ & $5 / 1 / 08$ & - & - & 3.4 & L & 28.0 & ND & ND & ND & Middle of screen. & MRMW5S-28-W-23273 \\
\hline MW5S & $15-55$ & $5 / 1 / 08$ & - & - & 4.0 & L & 52.0 & ND & ND & ND & Bottom of screen. & MRMW5S-52-W-23274 \\
\hline MW5S & $15-55$ & $10 / 21 / 08$ & 22.5 & 54.6 & 7.0 & $\mathrm{~L}$ & 35.0 & 1.7 & ND & ND & & MRMW5S-W-27625 \\
\hline MW5S & $15-55$ & $4 / 24 / 09$ & 22.1 & 54.6 & 5.5 & L & 38.4 & ND & ND & ND & & MRMW5S-W-27657 \\
\hline MW5S & $15-55$ & 9/3/09 & 17.6 & 54.6 & 5.5 & L & 36.3 & ND & ND & ND & & MRMW5S-W-29947 \\
\hline MW5S & $15-55$ & $4 / 7 / 10$ & 8.3 & 54.5 & 5.5 & L & 35.0 & ND & ND & ND & & MRMW5S-W-29987 \\
\hline MW5S & $15-55$ & $9 / 22 / 10$ & 19.3 & 55.0 & 6.5 & $\mathrm{~L}$ & 35.0 & ND & 9.4 & ND & & MRMW5S-W-30015 \\
\hline MW5S & $15-55$ & $4 / 20 / 11$ & 24.4 & 54.6 & 7.0 & L & 36.0 & 1.3 & ND & ND & & MRMW5S-W-30043 \\
\hline MW5S & $15-55$ & $10 / 4 / 11$ & 21.1 & 54.6 & 6.5 & L & 38.3 & ND & ND & ND & & MRMW5S-W-30072 \\
\hline MW6S & $10-25$ & $6 / 3 / 04$ & 3.3 & 26.9 & 45 & gal & - & ND & ND & ND & & MRMW6S-W-16465 \\
\hline MW6S & $10-25$ & $9 / 14 / 05$ & 4.7 & 26.9 & 43 & gal & - & ND & ND & ND & & MRMW6S-W-19263 \\
\hline MW6S & $10-25$ & $3 / 20 / 06$ & 5.4 & 26.9 & 43 & gal & - & ND & ND & ND & & MRMW6S-W-19990 \\
\hline MW6S & $10-25$ & $9 / 18 / 06$ & 5.5 & 26.9 & 27 & gal & - & ND & ND & ND & & MRMW6S-W-22486 \\
\hline MW6S & $10-25$ & $3 / 21 / 07$ & 5.4 & 26.9 & 30 & gal & - & ND & ND & ND & & MRMW6S-W-16486 \\
\hline MW6S & $10-25$ & $10 / 2 / 07$ & 5.0 & 26.9 & 31 & gal & - & ND & ND & ND & & MRMW6S-W-16583 \\
\hline MW6S & $10-25$ & $4 / 15 / 08$ & 5.2 & 26.9 & 2.5 & L & - & ND & ND & ND & & MRMW6S-W-23236 \\
\hline MW6S & $10-25$ & $10 / 20 / 08$ & 5.7 & 26.9 & 5.0 & L & 17.5 & ND & ND & ND & & MRMW6S-W-27626 \\
\hline MW6S & $10-25$ & $4 / 24 / 09$ & 6.2 & 26.9 & 12 & $\mathrm{~L}$ & 17.5 & ND & ND & ND & & MRMW6S-W-27658 \\
\hline MW6S & $10-25$ & $9 / 4 / 09$ & 5.9 & 26.9 & 5.4 & L & 17.5 & ND & ND & ND & & MRMW6S-W-29948 \\
\hline MW6S & $10-25$ & $4 / 6 / 10$ & 6.2 & 26.9 & 8.0 & L & 17.5 & ND & ND & ND & & MRMW6S-W-29988 \\
\hline MW6S & $10-25$ & $9 / 22 / 10$ & 5.5 & 26.9 & 8.8 & L & 17.5 & ND & ND & ND & & MRMW6S-W-30016 \\
\hline MW6S & $10-25$ & $4 / 20 / 11$ & 6.5 & 24.9 & 8.0 & $\bar{L}$ & 17.5 & ND & ND & ND & & MRMW6S-W-30044 \\
\hline MW6S & $10-25$ & $10 / 4 / 11$ & 6.5 & 26.9 & 6.0 & L & 17.5 & ND & ND & ND & & MRMW6S-W-30073 \\
\hline MW7S & $20-45$ & $6 / 3 / 04$ & 26.7 & 47.0 & 40 & gal & - & 18 & ND & ND & & MRMW7S-W-16466 \\
\hline MW7S & $20-45$ & $9 / 12 / 05$ & 17.6 & 46.9 & 55 & gal & - & 43 & 1.1 & ND & & MRMW7S-W-19258 \\
\hline
\end{tabular}


TABLE 3.2 (Cont.)

\begin{tabular}{|c|c|c|c|c|c|c|c|c|c|c|c|c|}
\hline \multirow[b]{2}{*}{ Location } & \multirow[b]{2}{*}{$\begin{array}{l}\text { Screen } \\
\text { Interval } \\
\text { (ft BGL) }\end{array}$} & \multirow[b]{2}{*}{$\begin{array}{l}\text { Sample } \\
\text { Date }\end{array}$} & \multirow[b]{2}{*}{$\begin{array}{l}\text { Depth to } \\
\text { Water } \\
\text { (ft BOTC) }\end{array}$} & \multirow[b]{2}{*}{$\begin{array}{l}\text { Depth of } \\
\text { well } \\
\text { (ft BTOC) }\end{array}$} & \multirow[b]{2}{*}{$\begin{array}{l}\text { Volume } \\
\text { Purged }\end{array}$} & \multirow[b]{2}{*}{$\begin{array}{l}\text { Purge } \\
\text { Units }\end{array}$} & \multirow{2}{*}{$\begin{array}{l}\text { Pump } \\
\text { Intake } \\
\text { Position } \\
\text { (ft BGL) }\end{array}$} & \multicolumn{3}{|c|}{ Concentration $(\mu \mathrm{g} / \mathrm{L})$} & \multirow[b]{2}{*}{ Comment } & \multirow[b]{2}{*}{ Sample } \\
\hline & & & & & & & & $\begin{array}{c}\text { Carbon } \\
\text { Tetrachloride }\end{array}$ & Chloroform & $\begin{array}{l}\text { Methylene } \\
\text { Chloride }\end{array}$ & & \\
\hline MW7S & $20-45$ & 3/22/06 & 22.5 & 47.0 & 48 & gal & - & 21 & $0.4 \mathrm{~J}$ & ND & & MRMW7S-W-20000 \\
\hline MW7S & $20-45$ & 9/19/06 & 20.9 & 47.0 & 56 & gal & - & 38 & $0.7 \mathrm{~J}$ & ND & & MRMW7S-W-22490 \\
\hline MW7S & $20-45$ & $3 / 20 / 07$ & 18.0 & 47.0 & 50 & gal & - & 16 & $0.4 \mathrm{~J}$ & ND & & MRMW7S-W-16481 \\
\hline MW7S & $20-45$ & $10 / 1 / 07$ & 12.4 & 47.0 & 70 & gal & - & 8.1 & $0.2 \mathrm{~J}$ & ND & & MRMW7S-W-16581 \\
\hline MW7S & $20-45$ & $4 / 14 / 08$ & 7.7 & 47.0 & 1.8 & $\mathrm{~L}$ & - & 10 & $0.3 \mathrm{~J}$ & ND & & MRMW7S-W-23237 \\
\hline MW7S & $20-45$ & $4 / 23 / 08$ & 7.8 & 47.0 & 11 & $\mathrm{~L}$ & - & 8.3 & $0.2 \mathrm{~J}$ & ND & & MRMW7S-W-23265 \\
\hline MW7S & $20-45$ & $10 / 20 / 08$ & 17.2 & 47.0 & 6.3 & L & 32.5 & 7.9 & ND & ND & & MRMW7S-W-27627 \\
\hline MW7S & $20-45$ & $4 / 23 / 09$ & 16.7 & 47.0 & 7.0 & $\bar{L}$ & 32.5 & 9.5 & ND & ND & & MRMW7S-W-27659 \\
\hline MW7S & $20-45$ & 9/3/09 & 13.8 & 47.0 & 9.0 & $\mathrm{~L}$ & 32.5 & 8.0 & ND & ND & & MRMW7S-W-29949 \\
\hline MW7S & $20-45$ & $4 / 6 / 10$ & 6.4 & 47.0 & 5.4 & $\bar{L}$ & 32.5 & 15 & $0.4 \mathrm{~J}$ & ND & & MRMW7S-W-29989 \\
\hline MW7S & $20-45$ & 9/23/10 & 14.2 & 45.0 & 6.0 & $\bar{L}$ & 32.5 & 6.6 & ND & ND & & MRMW7S-W-30017 \\
\hline MW7S & $20-45$ & $4 / 20 / 11$ & 19.7 & 46.7 & 6.0 & L & 32.5 & 4.4 & $0.2 \mathrm{~J}$ & ND & & MRMW7S-W-30045 \\
\hline MW7S & $20-45$ & $10 / 4 / 11$ & 7.1 & 47.0 & 7.0 & $\bar{L}$ & 32.5 & 11 & $0.3 \mathrm{~J}$ & ND & & MRMW7S-W-30074 \\
\hline MW7S & $20-45$ & 10/4/11 & 7.1 & 47.0 & 7.0 & $\mathrm{~L}$ & 32.5 & 12 & $0.3 \mathrm{~J}$ & ND & Replicate. & MRMW7SDUP-W-30083 \\
\hline MW8S & $10-25$ & $6 / 3 / 04$ & 3.7 & 26.8 & 45 & gal & - & ND & ND & ND & & MRMW8S-W-16464 \\
\hline MW8S & $10-25$ & 9/14/05 & 4.0 & 26.8 & 57 & gal & - & $0.9 \mathrm{~J}$ & ND & ND & & MRMW8S-W-19265 \\
\hline MW8S & $10-25$ & 3/20/06 & 4.6 & 26.4 & 43 & gal & - & $0.6 \mathrm{~J}$ & ND & $0.4 \mathrm{~J} \mathrm{~B}$ & & MRMW8S-W-19991 \\
\hline MW8S & $10-25$ & 9/19/06 & 4.8 & 26.8 & 45 & gal & - & 1.3 & ND & ND & & MRMW8S-W-22492 \\
\hline MW8S & $10-25$ & $3 / 20 / 07$ & 2.6 & 26.8 & 49 & gal & - & $0.6 \mathrm{~J}$ & ND & ND & & MRMW8S-W-16483 \\
\hline MW8S & $10-25$ & $10 / 2 / 07$ & 2.2 & 26.8 & 48 & gal & - & $0.8 \mathrm{~J}$ & ND & ND & & MRMW8S-W-16584 \\
\hline MW8S & $10-25$ & $4 / 15 / 08$ & 0.7 & 26.8 & 5.5 & $\mathrm{~L}$ & - & 1.1 & ND & ND & & MRMW8S-W-23238 \\
\hline MW8S & $10-25$ & 10/20/08 & 3.6 & 26.8 & 8.0 & L & 17.5 & 1.3 & ND & ND & & MRMW8S-W-27628 \\
\hline MW8S & $10-25$ & $4 / 23 / 09$ & 2.3 & 26.8 & 6.0 & $\bar{L}$ & 17.5 & ND & ND & ND & & MRMW8S-W-27660 \\
\hline MW8S & $10-25$ & $9 / 3 / 09$ & 2.9 & 26.8 & 8.5 & L & 17.5 & 1.9 & ND & ND & & MRMW8S-W-29950 \\
\hline MW8S & $10-25$ & $4 / 6 / 10$ & 1.1 & 26.8 & 8.0 & L & 17.5 & 1.7 & ND & ND & & MRMW8S-W-29990 \\
\hline MW8S & $10-25$ & 9/22/10 & 2.5 & 26.8 & 9.0 & $\mathrm{~L}$ & 17.5 & 1.6 & ND & ND & & MRMW8S-W-30018 \\
\hline MW8S & $10-25$ & $4 / 20 / 11$ & 2.7 & 26.8 & 9.0 & $\bar{L}$ & 17.5 & $0.2 \mathrm{~J}$ & ND & ND & & MRMW8S-W-30046 \\
\hline MW8S & $10-25$ & $10 / 4 / 11$ & 4.9 & 26.3 & 9.0 & L & 17.5 & $0.6 \mathrm{~J}$ & ND & ND & & MRMW8S-W-30075 \\
\hline MW9S & $38.83-53.83$ & $3 / 22 / 06$ & 20.2 & 58.6 & 20 & gal & - & ND & ND & ND & & MRMW9S-W-20004 \\
\hline MW9S & $38.83-53.83$ & $9 / 19 / 06$ & 18.9 & 59.0 & 22 & gal & - & ND & ND & ND & & MRMW9S-W-22494 \\
\hline MW9S & $38.83-53.83$ & 3/20/07 & 16.7 & 59.0 & 22 & gal & - & ND & ND & ND & & MRMW9S-W-16480 \\
\hline MW9S & $38.83-53.83$ & $10 / 1 / 07$ & 14.0 & 58.6 & 23 & gal & - & ND & ND & ND & & MRMW9S-W-16582 \\
\hline MW9S & $38.83-53.83$ & $4 / 14 / 08$ & 16.6 & 58.6 & 2.3 & $\mathrm{~L}$ & - & $0.8 \mathrm{~J}$ & ND & ND & & MRMW9S-W-23239 \\
\hline MW9S & $38.83-53.83$ & $10 / 20 / 08$ & 21.5 & 58.5 & 11 & $\bar{L}$ & 46.3 & 1.1 & ND & ND & & MRMW9S-W-27629 \\
\hline MW9S & $38.83-53.83$ & $4 / 23 / 09$ & 21.9 & 58.5 & 5.5 & $\mathrm{~L}$ & 46.3 & 1.0 & ND & ND & & MRMW9S-W-27661 \\
\hline MW9S & $38.83-53.83$ & $9 / 4 / 09$ & 20.0 & 58.5 & 5.0 & $\bar{L}$ & 46.3 & 1.4 & ND & ND & & MRMW9S-W-29951 \\
\hline MW9S & $38.83-53.83$ & $4 / 6 / 10$ & 16.5 & 58.5 & 6.0 & L & 46.3 & 1.9 & ND & ND & & MRMW9S-W-29991 \\
\hline MW9S & $38.83-53.83$ & 9/22/10 & 21.2 & 58.8 & 7.0 & $\bar{L}$ & 46.3 & 1.9 & ND & ND & & MRMW9S-W-30019 \\
\hline MW9S & $38.83-53.83$ & $4 / 20 / 11$ & 23.8 & 58.3 & 14 & $\bar{L}$ & 46.3 & $0.9 \mathrm{~J}$ & ND & ND & & MRMW9S-W-30047 \\
\hline MW9s & $38.83-53.83$ & $4 / 20 / 11$ & 23.8 & 58.3 & 14 & $\mathrm{~L}$ & 46.3 & 1.1 & ND & ND & Replicate. & MRMW9SDUP-W-30054 \\
\hline MW9S & $38.83-53.83$ & $10 / 4 / 11$ & 22.4 & 58.5 & 8.0 & L & 46.3 & $0.9 \mathrm{~J}$ & ND & ND & & MRMW9S-W-30076 \\
\hline MW10S & $30-45$ & 3/21/06 & 12.3 & 49.6 & 19 & gal & - & ND & ND & ND & & MRMW10S-W-19999 \\
\hline MW10S & $30-45$ & 9/18/06 & 11.1 & 49.6 & 20 & gal & - & ND & ND & ND & & MRMW10S-W-22489 \\
\hline MW10S & $30-45$ & $3 / 21 / 07$ & 10.8 & 49.6 & 20 & gal & - & ND & ND & ND & & MRMW10S-W-16485 \\
\hline MW10S & $30-45$ & 10/1/07 & 7.0 & 49.7 & 20 & gal & - & ND & ND & ND & & MRMW10S-W-16593 \\
\hline
\end{tabular}


TABLE 3.2 (Cont.)

\begin{tabular}{|c|c|c|c|c|c|c|c|c|c|c|c|c|}
\hline \multirow[b]{2}{*}{ Location } & \multirow{2}{*}{$\begin{array}{l}\text { Screen } \\
\text { Interval } \\
\text { (ft BGL) }\end{array}$} & \multirow[b]{2}{*}{$\begin{array}{l}\text { Sample } \\
\text { Date }\end{array}$} & \multirow{2}{*}{$\begin{array}{l}\text { Depth to } \\
\text { Water } \\
\text { (ft BOTC) }\end{array}$} & \multirow{2}{*}{$\begin{array}{l}\text { Depth of } \\
\text { well } \\
\text { (ft BTOC) }\end{array}$} & \multirow[b]{2}{*}{$\begin{array}{l}\text { Volume } \\
\text { Purged }\end{array}$} & \multirow[b]{2}{*}{$\begin{array}{l}\text { Purge } \\
\text { Units }\end{array}$} & \multirow{2}{*}{$\begin{array}{l}\text { Pump } \\
\text { Intake } \\
\text { Position } \\
\text { (ft BGL) }\end{array}$} & \multicolumn{3}{|c|}{ Concentration $(\mu \mathrm{g} / \mathrm{L})$} & \multirow[b]{2}{*}{ Comment } & \multirow[b]{2}{*}{ Sample } \\
\hline & & & & & & & & $\begin{array}{c}\text { Carbon } \\
\text { Tetrachloride }\end{array}$ & Chloroform & $\begin{array}{l}\text { Methylene } \\
\text { Chloride }\end{array}$ & & \\
\hline MW10S & $30-45$ & $4 / 14 / 08$ & 9.8 & 49.7 & 1.9 & $\mathrm{~L}$ & - & ND & ND & ND & & MRMW10S-W-23240 \\
\hline MW10S & $30-45$ & 10/20/08 & 13.7 & 49.7 & 5.4 & $\mathrm{~L}$ & 37.5 & ND & ND & ND & & MRMW10S-W-27630 \\
\hline MW10S & $30-45$ & $4 / 23 / 09$ & 13.6 & 45.0 & 7.5 & $\mathrm{~L}$ & 37.5 & ND & ND & ND & & MRMW10S-W-27662 \\
\hline MW10S & $30-45$ & 9/3/09 & 12.0 & 49.7 & 7.5 & $\mathrm{~L}$ & 37.5 & ND & ND & ND & & MRMW10S-W-29952 \\
\hline MW10S & $30-45$ & $4 / 6 / 10$ & 7.5 & 49.7 & 9.0 & L & 37.5 & ND & ND & ND & & MRMW10S-W-29992 \\
\hline MW10S & $30-45$ & $9 / 22 / 10$ & 11.7 & 49.7 & 11 & $\mathrm{~L}$ & 37.5 & ND & $0.3 \mathrm{~J}$ & ND & & MRMW10S-W-30020 \\
\hline MW10S & $30-45$ & $4 / 20 / 11$ & 13.7 & 49.3 & 7.3 & $\mathrm{~L}$ & 37.5 & ND & $0.2 \mathrm{~J}$ & ND & & MRMW10S-W-30048 \\
\hline MW10S & $30-45$ & $10 / 4 / 11$ & 12.5 & 49.7 & 8.0 & L & 37.5 & ND & ND & ND & & MRMW10S-W-30077 \\
\hline MW11S & $53-68$ & $3 / 22 / 06$ & 35.2 & 72.5 & 20 & gal & - & 39 & $0.9 \mathrm{~J}$ & ND & & MRMW11S-W-20001 \\
\hline MW11S & $53-68$ & 9/19/06 & 36.0 & 73.1 & 20 & gal & - & 53 & 1.0 & ND & & MRMW11S-W-22491 \\
\hline MW11S & $53-68$ & $3 / 20 / 07$ & 34.7 & 73.1 & 20 & gal & - & 37 & $0.8 \mathrm{~J}$ & ND & & MRMW11S-W-16479 \\
\hline MW11S & $53-68$ & $10 / 1 / 07$ & 31.6 & 73.0 & 20 & gal & - & 54 & 1.2 & ND & & MRMW11S-W-16594 \\
\hline MW11S & $53-68$ & $4 / 15 / 08$ & 29.9 & 72.7 & 5.5 & $\mathrm{~L}$ & - & 35 & $0.8 \mathrm{~J}$ & ND & April monitoring. & MRMW11S-W-23241 \\
\hline MW11S & $53-68$ & $4 / 22 / 08$ & 30.2 & 72.7 & 7.2 & $\mathrm{~L}$ & - & 42 & $0.9 \mathrm{~J}$ & ND & $\begin{array}{l}\text { Confirm low carbon } \\
\text { tetrachloride. }\end{array}$ & MRMW11S-W-23261 \\
\hline MW11S & $53-68$ & $10 / 20 / 08$ & 37.1 & 72.7 & 9.0 & $\mathrm{~L}$ & 60.5 & 42 & $0.9 \mathrm{~J}$ & ND & Low flow. & MRMW11S-W-27631 \\
\hline MW11S & $53-68$ & $10 / 21 / 08$ & - & - & - & - & - & 45 & $0.9 \mathrm{~J}$ & ND & Full purge. & MRMW11S-W-27651 \\
\hline MW11S & $53-68$ & $4 / 23 / 09$ & 38.1 & 72.7 & 5.0 & $\mathrm{~L}$ & 60.5 & 46 & 1.0 & ND & & MRMW11S-W-27663 \\
\hline MW11S & $53-68$ & 9/3/09 & 34.7 & 72.7 & 7.5 & $\mathrm{~L}$ & 60.5 & 39 & $0.9 \mathrm{~J}$ & ND & Low flow. & MRMW11S-W-29953 \\
\hline MW11S & $53-68$ & 9/4/09 & 35.0 & 72.7 & 72 & $\mathrm{~L}$ & 67.0 & 41 & $0.9 \mathrm{~J}$ & ND & Three well volumes. & MRMW11S-W-29973 \\
\hline MW11S & $53-68$ & $4 / 6 / 10$ & 29.5 & 72.7 & 6.5 & $\mathrm{~L}$ & 60.5 & 38 & 1.0 & ND & & MRMW11S-W-29993 \\
\hline MW11S & $53-68$ & $9 / 23 / 10$ & 34.8 & 72.7 & 7.0 & L & 60.5 & 28 & 1.0 & ND & & MRMW11S-W-30021 \\
\hline MW11S & $53-68$ & $4 / 20 / 11$ & 39.2 & 72.5 & 7.5 & $\mathrm{~L}$ & 60.5 & 38 & 1.1 & ND & & MRMW11S-W-30049 \\
\hline MW11S & $53-68$ & $10 / 4 / 11$ & 36.8 & 72.7 & 6.0 & L & 60.5 & 33 & $0.8 \mathrm{~J}$ & ND & & MRMW11S-W-30078 \\
\hline Isch & - & 2/19/04 & - & - & Pump ${ }^{e}$ & - & - & ND & ND & ND & & MRJR-W-16502 \\
\hline Isch & - & 9/14/05 & - & - & Pump & - & - & ND & ND & ND & & MRPRISCH-W-16513 \\
\hline Isch & - & $3 / 23 / 06$ & - & - & 20 & gal & - & ND & ND & ND & & MRISCH-W-19989 \\
\hline Isch & - & 9/19/06 & - & - & Pump & - & - & ND & ND & ND & & MRISCH-W-16531 \\
\hline Isch & - & $3 / 22 / 07$ & - & - & Pump & - & - & ND & ND & ND & & MRISCH-W-16564 \\
\hline Isch & - & $10 / 3 / 07$ & - & - & Pump & - & - & ND & ND & ND & & MRISCH-W-16590 \\
\hline Isch & - & $4 / 15 / 08$ & - & - & Pump & - & - & $0.4 \mathrm{~J}$ & ND & ND & & MRISCH-W-23242 \\
\hline Isch & - & $4 / 22 / 08$ & - & - & Pump & - & - & ND & ND & ND & & MRISCH-W-23262 \\
\hline Isch & - & $10 / 21 / 08$ & - & - & Pump & - & - & ND & ND & ND & & MRISCH-W-27632 \\
\hline Isch & - & $4 / 22 / 09$ & - & - & Pump & - & - & ND & ND & ND & & MRISCH-W-27664 \\
\hline Isch & - & $9 / 2 / 09$ & - & - & Pump & - & - & ND & ND & ND & & MRISCH-W-29954 \\
\hline Isch & - & $4 / 7 / 10$ & - & - & Pump & - & - & ND & ND & ND & & MRISCH-W-29994 \\
\hline Isch & - & $9 / 22 / 10$ & - & - & Pump & - & - & ND & ND & ND & & MRISCH-W-30022 \\
\hline Isch & - & $4 / 21 / 11$ & - & - & Pump & - & - & ND & ND & ND & & MRISCH-W-30050 \\
\hline Isch & - & 10/3/11 & - & - & Pump & - & - & ND & ND & ND & & MRIsch-W-30079 \\
\hline Rillinger & - & $6 / 4 / 04$ & - & - & Pump & - & - & ND & ND & ND & & MRPRIVRIL-W-16471 \\
\hline Rillinger & - & $9 / 14 / 05$ & - & - & Pump & - & - & 2.6 & $0.1 \mathrm{~J}$ & ND & & MRPRILL-W-16512 \\
\hline Rillinger & - & $3 / 19 / 06$ & - & - & Pump & - & - & ND & ND & $0.4 \mathrm{~J} \mathrm{~B}$ & & MRRILINGER-W-19988 \\
\hline Rillinger & - & 9/19/06 & - & - & Pump & - & - & ND & ND & ND & & MRRILI-W-16530 \\
\hline Rillinger & - & $3 / 29 / 07$ & - & - & Pump & - & - & 1.3 & 1.1 & ND & & MRRILINGER-W-16561 \\
\hline Rillinger & - & $10 / 3 / 07$ & - & - & Pump & - & - & $13^{\dagger}$ & $0.4 \mathrm{~J}$ & ND & & MRRILINGER-W-16591 \\
\hline
\end{tabular}


TABLE 3.2 (Cont.)

\begin{tabular}{|c|c|c|c|c|c|c|c|c|c|c|c|c|}
\hline \multirow[b]{2}{*}{ Location } & \multirow{2}{*}{$\begin{array}{l}\text { Screen } \\
\text { Interval } \\
\text { (ft BGL) }\end{array}$} & \multirow[b]{2}{*}{$\begin{array}{l}\text { Sample } \\
\text { Date }\end{array}$} & \multirow{2}{*}{$\begin{array}{l}\text { Depth to } \\
\text { Water } \\
\text { (ft BOTC) }\end{array}$} & \multirow{2}{*}{$\begin{array}{l}\text { Depth of } \\
\text { well } \\
\text { (ft BTOC) }\end{array}$} & \multirow[b]{2}{*}{$\begin{array}{l}\text { Volume } \\
\text { Purged }\end{array}$} & \multirow[b]{2}{*}{$\begin{array}{l}\text { Purge } \\
\text { Units }\end{array}$} & \multirow{2}{*}{$\begin{array}{l}\text { Pump } \\
\text { Intake } \\
\text { Position } \\
\text { (ft BGL) }\end{array}$} & \multicolumn{3}{|c|}{ Concentration $(\mu \mathrm{g} / \mathrm{L})$} & \multirow[b]{2}{*}{ Comment } & \multirow[b]{2}{*}{ Sample } \\
\hline & & & & & & & & $\begin{array}{c}\text { Carbon } \\
\text { Tetrachloride }\end{array}$ & Chloroform & $\begin{array}{l}\text { Methylene } \\
\text { Chloride }\end{array}$ & & \\
\hline Rillinger & - & $10 / 8 / 07$ & - & - & Pump & - & - & $0.4 \mathrm{~J}$ & ND & ND & & MRRILINGER-W-16592 \\
\hline Rillinger & - & 1/11/08 & - & - & Pump & - & - & 6.2 & $0.5 \mathrm{~J}$ & ND & & MORIL-W-11108 \\
\hline Rillinger & - & $4 / 15 / 08$ & - & - & Pump & - & - & 9.9 & $0.4 \mathrm{~J}$ & ND & & MRRILINGER-W-23243 \\
\hline Rillinger & - & 10/21/08 & - & - & Pump & - & - & $0.9 \mathrm{~J}$ & ND & ND & & MRRILLINGER-W-27633 \\
\hline Rillinger & - & $4 / 22 / 09$ & - & - & Pump & - & - & 1.2 & ND & ND & & MRRILLINGER-W-27665 \\
\hline Rillinger & - & $9 / 2 / 09$ & - & - & Pump & - & - & 1.0 & ND & ND & & MRRILLINGER-W-29955 \\
\hline Rillinger & - & $4 / 7 / 10$ & - & - & Pump & - & - & $0.8 \mathrm{~J}$ & ND & ND & & MRRILLINGER-W-29995 \\
\hline Rillinger & - & 9/22/10 & - & - & Pump & - & - & 1.3 & ND & ND & & MRRILLINGER-W-30023 \\
\hline Rillinger & - & $4 / 21 / 11$ & - & - & Pump & - & - & $0.7 \mathrm{~J}$ & ND & ND & & MRRILLINGER-W-30051 \\
\hline Rillinger & - & 10/3/11 & - & - & Pump & - & - & $0.7 \mathrm{~J}$ & ND & ND & & MRRillinger-W-30080 \\
\hline Stone & $43^{g}$ & $6 / 4 / 04$ & 23.4 & - & \multicolumn{2}{|c|}{ Purged dry. } & - & 10 & ND & ND & & MRPRIVSTON-W-16475 \\
\hline Stone & 43 & 9/14/05 & 17.2 & 40.0 & - & - & - & 2.6 & $0.3 \mathrm{~J}$ & ND & & MRPRSTON-W-16511 \\
\hline Stone & 43 & 3/19/06 & 17.4 & 40.0 & 100 & gal & - & 14 & $0.8 \mathrm{~J}$ & $0.4 \mathrm{~J} \mathrm{~B}$ & & MRSTONE-W-19987 \\
\hline Stone & 43 & 9/19/06 & 18.6 & 38.8 & 41 & gal & - & 2.1 & ND & ND & & MRSTONE-W-16529 \\
\hline Stone & 43 & $3 / 22 / 07$ & 20.6 & 38.8 & 56 & gal & - & 5.4 & $0.3 \mathrm{~J}$ & ND & & MRSTONE-W-16560 \\
\hline Stone & 43 & $10 / 3 / 07$ & 14.6 & 38.6 & 72 & gal & - & 2.8 & ND & ND & & MRSTONE-W-16589 \\
\hline Stone & 43 & $4 / 15 / 08$ & - & 38.9 & - & - & - & $0.9 \mathrm{~J}$ & ND & ND & & MRSTONE-W-23244 \\
\hline Stone & 43 & 10/21/08 & - & - & 5.0 & gal & - & 3.0 & ND & ND & & MRSTONE-W-27634 \\
\hline Stone & 43 & $4 / 23 / 09$ & - & - & 5.0 & gal & - & 1.1 & ND & ND & & MRSTONE-W-27666 \\
\hline Stone & 43 & 9/2/09 & - & - & 5.0 & gal & - & $0.9 \mathrm{~J}$ & ND & ND & & MRSTONE-W-29956 \\
\hline Stone & 43 & $4 / 7 / 10$ & - & - & 5.0 & gal & - & $0.5 \mathrm{~J}$ & ND & ND & & MRSTONE-W-29996 \\
\hline Stone & 43 & 9/22/10 & - & - & 5.0 & gal & - & $0.6 \mathrm{~J}$ & 1.8 & ND & & MRSTONE-W-30024 \\
\hline Stone & 43 & $4 / 21 / 11$ & - & - & 5.0 & gal & - & 1.8 & $0.9 \mathrm{~J}$ & ND & & MRSTONE-W-30052 \\
\hline Stone & 43 & 10/3/11 & - & - & 5.0 & gal & - & 1.5 & ND & ND & & MRStone-W-30081 \\
\hline TD12 & $27-67$ & $4 / 22 / 08$ & - & - & - & - & - & ND & ND & ND & Grimm irrigation well. & MRTD12-W-23264 \\
\hline TD12 & $27-67$ & 10/20/08 & - & - & - & - & - & ND & ND & ND & Grimm irrigation well. & MRTD12-W-27635 \\
\hline TD12 & $27-67$ & $4 / 23 / 09$ & - & - & - & - & - & ND & ND & ND & Tile drain into creek. & MRTD12-W-27667 \\
\hline TD12 & $27-67$ & 9/3/09 & - & - & - & - & - & ND & ND & ND & Overflow before catchment. & MRTD12-W-29957 \\
\hline TD12 & $27-67$ & $4 / 6 / 10$ & - & - & - & - & - & ND & ND & ND & & MRTD12-W-29997 \\
\hline TD12 & $27-67$ & 9/22/10 & - & - & - & - & - & ND & ND & ND & & MRTD12-W-30025 \\
\hline TD12 & $27-67$ & $4 / 20 / 11$ & - & - & - & - & - & ND & ND & ND & & MRTD12-W-30053 \\
\hline TD12 & $27-67$ & 10/3/11 & - & - & - & - & - & ND & ND & ND & & MRTD12-W-30082 \\
\hline
\end{tabular}

ND, not detected at an instrument detection limit of $0.1 \mu \mathrm{g} / \mathrm{L}$.

b Qualifier J indicates an estimated concentration below the method quantitation limit of $1.0 \mu \mathrm{g} / \mathrm{L}$.

c Qualifier B indicates that the compound was present in the associated method blank.

d Qualifier R indicates that the contaminant was present in the associated equipment rinsate. Resampling confirmed that the well was free of contamination.

e The well's dedicated pump was used for sampling. The pump was allowed to run before the sample was collected.

f Sample collected after recent reactivation of well. Well resampled on 10/8/07 and 1/11/08.

g Total depth 
TABLE 3.3 Field measurements for groundwater samples collected in 2003-2011. Shading indicates sample collection with the low-flow procedure.

\begin{tabular}{|c|c|c|c|c|c|c|c|c|c|c|c|c|c|c|c|}
\hline \multirow[b]{2}{*}{ Location } & \multirow{2}{*}{$\begin{array}{l}\text { Screen } \\
\text { Interval } \\
\text { (ft BGL) }\end{array}$} & \multirow{2}{*}{$\begin{array}{l}\text { Sample } \\
\text { Date }\end{array}$} & \multicolumn{2}{|c|}{ Depth (ft BTOC) } & \multirow{2}{*}{$\begin{array}{l}\text { Volume } \\
\text { Purged }\end{array}$} & \multirow{2}{*}{$\begin{array}{l}\text { Purge } \\
\text { Units }\end{array}$} & \multirow{2}{*}{$\begin{array}{l}\text { Pump } \\
\text { Intake } \\
\text { Position } \\
\text { (ft BGL) }\end{array}$} & \multirow{2}{*}{$\begin{array}{c}\text { Temperature } \\
\left({ }^{\circ} \mathrm{C}\right)\end{array}$} & \multirow[b]{2}{*}{$\mathrm{pH}$} & \multirow{2}{*}{$\begin{array}{l}\text { Conductivity } \\
(\mu \mathrm{S} / \mathrm{cm})\end{array}$} & \multirow{2}{*}{$\begin{array}{c}\text { Dissolved } \\
\text { Oxygen } \\
\text { (mg/L) }\end{array}$} & \multirow{2}{*}{$\begin{array}{l}\text { ORP } \\
(\mathrm{mV})\end{array}$} & \multirow{2}{*}{$\begin{array}{l}\text { Iron II } \\
\text { (mg/L) }\end{array}$} & \multirow{2}{*}{$\begin{array}{c}\text { Carbon } \\
\text { Dioxide } \\
\text { (mg/L) }\end{array}$} & \multirow[b]{2}{*}{ Sample } \\
\hline & & & Water & Well & & & & & & & & & & & \\
\hline MW1S & $11-51$ & $10 / 23 / 03$ & 30.4 & 54.0 & 70 & gal & - & 14.6 & 7.14 & 933 & $--^{a}$ & 13 & - & - & MRMW1S-W-16422 \\
\hline MW1S & $11-51$ & $6 / 2 / 04$ & 27.0 & 53.9 & 53 & gal & - & 14.4 & 7.16 & 970 & - & - & - & - & MRMW1S-W-16461 \\
\hline MW1S & $11-51$ & 9/13/05 & 24.2 & 53.9 & 57 & gal & - & 15.3 & 6.95 & 1174 & 7.17 & 200 & 0 & 55 & MRMW1S-W-19259 \\
\hline MW1S & $11-51$ & $3 / 22 / 06$ & 29.0 & 54.0 & 48 & gal & - & 15.5 & 7.23 & 927 & 9.94 & 220 & 0.01 & 40 & MRMW1S-W-20008 \\
\hline MW1S & $11-51$ & 9/20/06 & 26.8 & 54.0 & 55 & gal & - & 15.7 & 7.12 & 973 & 7.52 & - & 0.03 & 40 & MRMW1S-W-22495 \\
\hline MW1S & $11-51$ & $3 / 21 / 07$ & 25.8 & 54.0 & 55 & gal & - & 16.6 & 6.48 & 960 & 5.45 & 88 & 0 & 40 & MRMW1S-W-16488 \\
\hline MW1S & $11-51$ & $10 / 1 / 07$ & 21.7 & 54.0 & 63 & gal & - & 16.0 & 6.80 & 886 & 6.79 & 128 & 0 & 30 & MRMW1S-W-16595 \\
\hline MW1S & $11-51$ & $4 / 14 / 08$ & 16.2 & 54.0 & 5.5 & $\mathrm{~L}$ & - & 13.9 & 7.09 & 1237 & 6.38 & 118 & 0.02 & - & MRMW1S-W-23230 \\
\hline MW1S & $11-51$ & $4 / 22 / 08$ & 16.0 & 54.0 & 6.3 & $\mathrm{~L}$ & - & 15.6 & 6.96 & 1230 & 6.10 & 133 & - & - & MRMW1S-W-23259 \\
\hline MW1S & $11-51$ & $5 / 1 / 08$ & - & - & 3.2 & L & 22.0 & 17.1 & 7.11 & 801 & 3.34 & 104 & - & - & MRMW1S-22-W-23275 \\
\hline MW1S & $11-51$ & $5 / 1 / 08$ & - & - & 3.2 & L & 27.0 & 17.0 & 7.10 & 820 & 3.40 & 102 & - & - & MRMW1S-27-W-23276 \\
\hline MW1S & $11-51$ & $5 / 1 / 08$ & - & - & 4.3 & $\mathrm{~L}$ & 48.0 & 16.3 & 7.00 & 1301 & 3.03 & 118 & - & - & MRMW1S-48-W-23277 \\
\hline MW1S & $11-51$ & $10 / 20 / 08$ & 25.8 & 54.0 & 6.0 & L & 31.0 & 14.0 & 6.84 & 1265 & 5.40 & 103 & 0 & - & MRMW1S-W-27620 \\
\hline MW1S & $11-51$ & $10 / 21 / 08$ & - & - & $T W V^{b}$ & - & - & 14.8 & 7.02 & 978 & - & - & 0 & - & MRMW1S-W-27649 \\
\hline MW1S & $11-51$ & $4 / 24 / 09$ & 24.4 & 54.0 & 5.0 & $\mathrm{~L}$ & 39.2 & 16.2 & 7.00 & 986 & 8.93 & 75 & 0.04 & - & MRMW1S-W-27652 \\
\hline MW1S & $11-51$ & 9/3/09 & 19.0 & 54.0 & 8.0 & L & 35.0 & 16.0 & 6.94 & 1334 & 7.08 & 28 & 0.04 & - & MRMW1S-W-29942 \\
\hline MW1S & $11-51$ & 9/4/09 & 19.3 & 51.2 & 244 & L & 50.0 & 15.0 & 6.92 & 950 & 7.73 & 61 & - & - & MRMW1S-W-29971 \\
\hline MW1S & $11-51$ & $4 / 7 / 10$ & 11.7 & 51.3 & 7.0 & $\mathrm{~L}$ & 16.6 & 9.6 & 7.01 & 695 & 8.66 & 233 & 0 & - & MRMW1ST-W-29981 \\
\hline MW1S & $11-51$ & $4 / 7 / 10$ & 11.6 & 51.3 & 6.0 & $\mathrm{~L}$ & 31.5 & 11.9 & 6.96 & 918 & 8.42 & 246 & 0 & - & MRMW1SM-W-29980 \\
\hline MW1S & $11-51$ & $4 / 7 / 10$ & 11.7 & 51.3 & 6.0 & $\mathrm{~L}$ & 46.3 & 11.7 & 6.95 & 965 & 8.29 & 251 & 0.01 & - & MRMW1SB-W-29979 \\
\hline MW1S & $11-51$ & $4 / 7 / 10$ & 11.5 & 51.3 & 80 & gal & 49.0 & 14.0 & 7.18 & 687 & 8.86 & 211 & 0.03 & - & MRMW1S3X-W-29982 \\
\hline MW1S & $11-51$ & $9 / 22 / 10$ & 19.9 & 54.0 & 10 & $\mathrm{~L}$ & 31.0 & 17.9 & 7.01 & 1337 & 6.17 & 134 & 0.07 & - & MRMW1S-W-30010 \\
\hline MW1S & $11-51$ & $4 / 20 / 11$ & 23.5 & 54.0 & 6.5 & L & 37.0 & 13.8 & 6.96 & 866 & 6.39 & 55 & 0 & - & MRMW1S-W-30038 \\
\hline MW1S & $11-51$ & $10 / 4 / 11$ & 21.8 & 54.0 & 8.0 & L & 34.9 & 15.4 & 7.65 & 1166 & 5.72 & 98 & 0 & - & MRMW1S-W-30067 \\
\hline MW1S & $11-51$ & 10/12/11 & 22.1 & 54.0 & 6.0 & $\mathrm{~L}$ & 36.6 & 16.3 & 7.24 & 1279 & 5.76 & 323 & - & - & MRMW1S-W-30091 \\
\hline MW1S & $11-51$ & 10/12/11 & 22.4 & 54.0 & 65 & gal & 49.0 & 16.7 & 7.36 & 905 & 6.18 & 270 & - & - & MRMW1S3X-W-30092 \\
\hline MW1D & $63-88$ & $10 / 22 / 03$ & 28.4 & 88.5 & 92 & gal & - & 14.9 & 6.87 & 2620 & - & 25 & - & - & MRMW1D-W-16421 \\
\hline MW1D & $63-88$ & $6 / 2 / 04$ & 26.8 & 88.6 & 140 & gal & - & 13.9 & 6.87 & 2460 & - & - & - & - & MRMW1D-W-16458 \\
\hline MW1D & $63-88$ & $9 / 13 / 05$ & 23.7 & 88.6 & 200 & gal & - & 15.5 & 6.56 & 2470 & - & - & - & - & MRMW1D-W-16518 \\
\hline MW1D & $63-88$ & $3 / 19 / 06$ & 26.9 & 88.6 & 112 & gal & - & 12.9 & 6.95 & 2460 & 5.11 & 230 & 0 & - & MRMW1D-W-19986 \\
\hline MW1D & $63-88$ & 9/20/06 & 25.5 & 88.8 & 125 & gal & - & 12.5 & 6.93 & 2690 & - & - & - & - & MRMW1D-W-16532 \\
\hline MW1D & $63-88$ & $3 / 21 / 07$ & 25.8 & 88.8 & 125 & gal & - & 15.3 & 6.39 & 2540 & 0.08 & 12 & 0.39 & 40 & MRMW1D-W-16487 \\
\hline MW1D & $63-88$ & $10 / 1 / 07$ & 22.8 & 89.4 & 130 & gal & - & 16.3 & 6.60 & 2230 & 6.79 & 5 & 0.44 & 45 & MRMW1D-W-16596 \\
\hline MW1D & $63-88$ & $4 / 14 / 08$ & 29.5 & 89.0 & 6.0 & L & - & 14.6 & 6.99 & 2637 & 0.50 & 32 & 0.73 & - & MRMW1D-W-23231 \\
\hline MW1D & $63-88$ & $10 / 20 / 08$ & 30.4 & 89.0 & 7.0 & L & 75.5 & 13.4 & 6.83 & 2556 & 0.24 & 21 & 0.37 & - & MRMW1D-W-27621 \\
\hline MW1D & $63-88$ & $4 / 24 / 09$ & 31.0 & 89.0 & 7.0 & $\bar{L}$ & 75.5 & 16.7 & 6.92 & 2419 & 0.37 & 22 & 0.28 & - & MRMW1D-W-27653 \\
\hline MW1D & $63-88$ & 9/3/09 & 27.1 & 89.0 & 6.5 & $\mathrm{~L}$ & 75.5 & 16.9 & 7.00 & 2200 & 0.59 & 16 & 0.27 & - & MRMW1D-W-29943 \\
\hline MW1D & $63-88$ & $4 / 6 / 10$ & 24.7 & 89.0 & 8.5 & L & 75.5 & 17.8 & 6.39 & 2274 & 0.15 & 33 & 0.01 & - & MRMW1D-W-29983 \\
\hline MW1D & $63-88$ & $9 / 22 / 10$ & 27.9 & 89.0 & 9.0 & L & 75.5 & 17.6 & 7.01 & 2492 & 0.31 & 61 & 0.04 & - & MRMW1D-W-30011 \\
\hline MW1D & $63-88$ & $4 / 20 / 11$ & 30.2 & 89.0 & 5.5 & L & 75.5 & 13.5 & 6.98 & 1951 & 0.33 & -2 & 0.20 & - & MRMW1D-W-30039 \\
\hline MW1D & $63-88$ & $10 / 4 / 11$ & 29.1 & 89.0 & 6.5 & L & 75.5 & 14.9 & 7.66 & 2011 & 0.17 & 22 & 0.09 & - & MRMW1D-W-30068 \\
\hline MW2S & $13-53$ & $10 / 22 / 03$ & 42.2 & 53.4 & $\mathrm{PDS}^{\mathrm{c}}$ & - & - & 16.2 & 6.86 & 875 & - & 20 & - & - & MRMW02-W-16419 \\
\hline MW2S & $13-53$ & $6 / 2 / 04$ & 37.4 & 53.3 & 31 & gal & - & 16.9 & 7.07 & 861 & - & - & - & - & MRMW2S-W-16459 \\
\hline MW2S & $13-53$ & $9 / 14 / 05$ & 33.7 & 53.3 & 38 & gal & - & 15.2 & 6.94 & 801 & 7.85 & 142 & - & 65 & MRMW2S-W-19264 \\
\hline MW2S & $13-53$ & $3 / 21 / 06$ & 40.9 & 53.3 & 27 & gal & - & 13.0 & 7.07 & 863 & 9.40 & 262 & 0.14 & 25 & MRMW2S-W-19992 \\
\hline
\end{tabular}


TABLE 3.3 (Cont.)

\begin{tabular}{|c|c|c|c|c|c|c|c|c|c|c|c|c|c|c|c|}
\hline \multirow[b]{2}{*}{ Location } & \multirow{2}{*}{$\begin{array}{l}\text { Screen } \\
\text { Interval } \\
\text { (ft BGL) }\end{array}$} & \multirow{2}{*}{$\begin{array}{l}\text { Sample } \\
\text { Date }\end{array}$} & \multicolumn{2}{|c|}{ Depth (ft BTOC) } & \multirow{2}{*}{$\begin{array}{l}\text { Volume } \\
\text { Purged }\end{array}$} & \multirow{2}{*}{$\begin{array}{l}\text { Purge } \\
\text { Units }\end{array}$} & \multirow{2}{*}{$\begin{array}{l}\text { Pump } \\
\text { Intake } \\
\text { Position } \\
\text { (ft BGL) }\end{array}$} & \multirow{2}{*}{$\begin{array}{c}\text { Temperature } \\
\left({ }^{\circ} \mathrm{C}\right)\end{array}$} & \multirow[b]{2}{*}{$\mathrm{pH}$} & \multirow{2}{*}{$\begin{array}{c}\text { Conductivity } \\
(\mu \mathrm{S} / \mathrm{cm})\end{array}$} & \multirow{2}{*}{$\begin{array}{c}\text { Dissolved } \\
\text { Oxygen } \\
\text { (mg/L) }\end{array}$} & \multirow{2}{*}{$\begin{array}{l}\text { ORP } \\
(\mathrm{mV})\end{array}$} & \multirow{2}{*}{$\begin{array}{l}\text { Iron II } \\
\text { (mg/L) }\end{array}$} & \multirow{2}{*}{$\begin{array}{l}\text { Carbon } \\
\text { Dioxide } \\
\text { (mg/L) }\end{array}$} & \multirow[b]{2}{*}{ Sample } \\
\hline & & & Water & Well & & & & & & & & & & & \\
\hline MW2S & $13-53$ & 9/18/06 & 36.5 & 53.3 & 28 & gal & - & 13.6 & 6.99 & 844 & 6.81 & 69 & 0 & 80 & MRMW2S-W-22488 \\
\hline MW2S & $13-53$ & $3 / 22 / 07$ & 35.8 & 53.3 & 35 & gal & - & 15.2 & 6.40 & 790 & 5.82 & 69 & 0 & 30 & MRMW2S-W-16559 \\
\hline MW2S & $13-53$ & $10 / 3 / 07$ & 31.2 & 53.4 & 44 & gal & - & 16.8 & 6.97 & 703 & 6.70 & 269 & 0.01 & 30 & MRMW2S-W-16587 \\
\hline MW2S & $13-53$ & $4 / 15 / 08$ & 23.6 & 53.4 & 2.2 & $\mathrm{~L}$ & - & 11.7 & 7.23 & 742 & 3.22 & 75 & 0 & - & MRMW2S-W-23232 \\
\hline MW2S & $13-53$ & $10 / 21 / 08$ & 33.7 & 53.5 & 5.0 & L & 33.0 & 13.4 & 7.08 & 745 & 8.55 & 104 & 0 & - & MRMW2S-W-27622 \\
\hline MW2S & $13-53$ & 10/21/08 & - & - & TWV & - & - & - & - & - & - & - & - & - & MRMW2S-W-27652 \\
\hline MW2S & $13-53$ & $4 / 23 / 09$ & 33.2 & 53.5 & 6.5 & $\mathrm{~L}$ & 43.4 & 15.2 & 6.97 & 755 & 9.19 & 26 & 0 & - & MRMW2S-W-27654 \\
\hline MW2S & $13-53$ & $9 / 3 / 09$ & 29.4 & 53.5 & 5.4 & L & 41.2 & 15.1 & 7.15 & 690 & 8.80 & 32 & 0.01 & - & MRMW2S-W-29944 \\
\hline MW2S & $13-53$ & $4 / 6 / 10$ & 18.6 & 52.4 & 6.5 & L & 36.0 & 17.1 & 6.95 & 681 & 6.85 & 37 & 0.12 & - & MRMW2S-W-29984 \\
\hline MW2S & $13-53$ & $9 / 22 / 10$ & 31.3 & 53.0 & 7.0 & $\mathrm{~L}$ & 33.0 & 16.1 & 7.03 & 723 & 7.16 & 142 & 0 & - & MRMW2S-W-30012 \\
\hline MW2S & $13-53$ & $4 / 20 / 11$ & 36.0 & 53.5 & 8.0 & $\mathrm{~L}$ & 44.5 & 13.4 & 6.91 & 615 & 7.07 & 103 & 0.05 & - & MRMW2S-W-30040 \\
\hline MW2S & $13-53$ & $10 / 4 / 11$ & 32.7 & 53.5 & 7.5 & $\mathrm{~L}$ & 42.6 & 15.3 & 7.71 & 592 & 6.89 & 120 & 0.01 & - & MRMW2S-W-30069 \\
\hline MW3S & $18-48$ & $10 / 23 / 03$ & 36.5 & 47.8 & 73 & gal & - & 16.8 & 7.23 & 655 & - & 6 & - & - & MRMW03-W-16423 \\
\hline MW3S & $18-48$ & $6 / 2 / 04$ & 30.7 & 47.5 & 34 & gal & - & 14.2 & 7.23 & 664 & - & - & - & - & MRMW3S-W-16462 \\
\hline MW3S & $18-48$ & 9/13/05 & 25.6 & 47.6 & 50 & gal & - & 14.6 & 7.13 & 663 & 8.82 & 223 & 0 & 100 & MRMW3S-W-19261 \\
\hline MW3S & $18-48$ & $3 / 23 / 06$ & 35.6 & 47.7 & 28 & gal & - & 8.9 & 7.16 & 662 & 6.74 & 269 & 0.08 & 25 & MRMW3S-W-19994 \\
\hline MW3S & $18-48$ & 9/20/06 & 29.4 & 47.8 & 22 & gal & - & 12.9 & 7.15 & 669 & 7.64 & 105 & 0 & - & MRMW3S-W-22496 \\
\hline MW3S & $18-48$ & $3 / 22 / 07$ & 26.2 & 47.8 & 45 & gal & - & 15.0 & 6.44 & 578 & 5.90 & 261 & 0.17 & 30 & MRMW3S-W-16563 \\
\hline MW3S & $18-48$ & $10 / 3 / 07$ & 22.7 & 47.9 & 50 & gal & - & 15.3 & 6.97 & 594 & 0.38 & 282 & 0 & 20 & MRMW3S-W-16585 \\
\hline MW3S & $18-48$ & $4 / 14 / 08$ & 17.0 & 47.8 & 3.3 & $\mathrm{~L}$ & - & 13.7 & 7.17 & 693 & 3.52 & 165 & 0 & - & MRMW3S-W-23233 \\
\hline MW3S & $18-48$ & $4 / 22 / 08$ & 15.8 & 47.8 & 6.5 & L & - & 16.0 & 6.99 & 685 & 6.71 & 155 & - & - & MRMW3S-W-23260 \\
\hline MW3S & $18-48$ & $5 / 1 / 08$ & - & - & 2.6 & L & 26.0 & 13.2 & 7.17 & 675 & 3.83 & 161 & - & - & MRMW3S-26-W-23269 \\
\hline MW3S & $18-48$ & $5 / 1 / 08$ & - & - & 2.8 & $\mathrm{~L}$ & 38.0 & 12.7 & 7.12 & 671 & 4.21 & 193 & - & - & MRMW3S-38-W-23270 \\
\hline MW3S & $18-48$ & $5 / 1 / 08$ & - & - & 3.2 & L & 45.0 & 12.6 & 7.03 & 675 & 4.57 & 205 & - & - & MRMW3S-45-W-23271 \\
\hline MW3S & $18-48$ & $10 / 21 / 08$ & 27.0 & 47.8 & 4.2 & L & 33.0 & 12.6 & 7.17 & 673 & 6.42 & 115 & 0 & - & MRMW3S-W-27623 \\
\hline MW3S & $18-48$ & $10 / 21 / 08$ & - & - & TWV & - & - & 14.3 & 7.11 & 522 & - & - & 0 & - & MRMW3S-W-27650 \\
\hline MW3S & $18-48$ & $4 / 23 / 09$ & 26.7 & 47.8 & 5.0 & $\mathrm{~L}$ & 37.3 & 17.0 & 7.06 & 662 & 9.40 & -63 & 0.01 & - & MRMW3S-W-27655 \\
\hline MW3S & $18-48$ & $9 / 3 / 09$ & 22.4 & 47.8 & 5.5 & L & 35.2 & 16.3 & 7.28 & 640 & 7.69 & 12 & 0.01 & - & MRMW3S-W-29945 \\
\hline MW3S & $18-48$ & 9/4/09 & 22.6 & 47.8 & 190 & L & 46.0 & 14.2 & 6.57 & 659 & 9.09 & 95 & - & - & MRMW3S-W-29972 \\
\hline MW3S & $18-48$ & $4 / 6 / 10$ & 12.1 & 47.8 & 6.0 & L & 33.0 & 16.0 & 6.99 & 611 & 8.20 & 8 & 0 & - & MRMW3S-W-29985 \\
\hline MW3S & $18-48$ & $9 / 23 / 10$ & 23.7 & 47.8 & 7.0 & L & 33.0 & 15.8 & 7.22 & 674 & 11.00 & 150 & 0.05 & - & MRMW3S-W-30013 \\
\hline MW3S & $18-48$ & $4 / 20 / 11$ & 32.1 & 47.8 & 6.2 & $\mathrm{~L}$ & 40.0 & 13.3 & 7.11 & 514 & 6.64 & 61 & 0.02 & - & MRMW3S-W-30041 \\
\hline MW3S & $18-48$ & $10 / 4 / 11$ & 26.0 & 47.8 & 7.0 & L & 37.0 & 15.8 & 7.78 & 563 & 5.99 & 115 & 0 & - & MRMW3S-W-30070 \\
\hline MW4S & $17-47$ & $10 / 21 / 03$ & 46.4 & 47.8 & PDS & - & - & - & 7.17 & 758 & - & - & - & - & MRMW04-W-16418 \\
\hline MW4S & $17-47$ & $6 / 4 / 04$ & 43.2 & 47.8 & 10 & gal & - & 15.4 & 6.93 & 769 & - & - & - & - & MRMW4S-W-16470 \\
\hline MW4S & $17-47$ & 9/14/05 & 36.2 & 47.8 & 8.0 & gal & - & 15.4 & 7.30 & 751 & 8.00 & 174 & 0 & 50 & MRMW4S-W-19262 \\
\hline MW4S & $17-47$ & $3 / 21 / 06$ & 44.6 & 47.7 & 6.0 & gal & - & 6.7 & 7.25 & 729 & 10.90 & 154 & 0 & 25 & MRMW4S-W-19993 \\
\hline MW4S & $17-47$ & $9 / 18 / 06$ & 41.6 & 47.8 & 5.3 & gal & - & 13.1 & 7.25 & 728 & 8.05 & 41 & 0 & 50 & MRMW4S-W-22487 \\
\hline MW4S & $17-47$ & $3 / 22 / 07$ & 38.7 & 47.8 & 6.0 & gal & - & 14.2 & 6.53 & 765 & 5.91 & 78 & 0.1 & 25 & MRMW4S-W-16562 \\
\hline MW4S & $17-47$ & $10 / 3 / 07$ & 31.1 & 47.7 & 30 & gal & - & 16.4 & 6.95 & 715 & 7.40 & 281 & 0.1 & 30 & MRMW4S-W-16586 \\
\hline MW4S & $17-47$ & $1 / 11 / 08$ & - & - & - & - & - & 11.3 & 7.56 & 757 & - & - & - & - & MOMW4S-W-011108 \\
\hline MW4S & $17-47$ & $4 / 14 / 08$ & 26.3 & 47.9 & 2.5 & L & - & 13.1 & 7.28 & 783 & 3.80 & 213 & 0 & - & MRMW4S-W-23234 \\
\hline MW4S & $17-47$ & $10 / 20 / 08$ & 36.7 & 47.8 & 8.0 & gal & - & 14.5 & 7.16 & 756 & 8.71 & 104 & 0 & - & MRMW4S-W-27624 \\
\hline MW4S & $17-47$ & $4 / 23 / 09$ & 41.5 & 47.8 & 5.0 & $\mathrm{~L}$ & 44.7 & 15.8 & 7.16 & 717 & 9.45 & 22 & 0.05 & - & MRMW4S-W-27656 \\
\hline MW4S & $17-47$ & $9 / 4 / 09$ & 31.6 & 47.8 & 6.0 & $\mathrm{~L}$ & 39.3 & 15.0 & 7.13 & 731 & 8.23 & 80 & 0.04 & - & MRMW4S-W-29946 \\
\hline MW4S & $17-47$ & $4 / 6 / 10$ & 21.8 & 47.9 & 5.5 & L & 34.8 & 16.5 & 7.07 & 629 & 8.07 & -1 & 0 & - & MRMW4S-W-29986 \\
\hline
\end{tabular}


TABLE 3.3 (Cont.)

\begin{tabular}{|c|c|c|c|c|c|c|c|c|c|c|c|c|c|c|c|}
\hline \multirow[b]{2}{*}{ Location } & \multirow{2}{*}{$\begin{array}{l}\text { Screen } \\
\text { Interval } \\
\text { (ft BGL) }\end{array}$} & \multirow{2}{*}{$\begin{array}{l}\text { Sample } \\
\text { Date }\end{array}$} & \multicolumn{2}{|c|}{ Depth (ft BTOC) } & \multirow{2}{*}{$\begin{array}{l}\text { Volume } \\
\text { Purged }\end{array}$} & \multirow{2}{*}{$\begin{array}{l}\text { Purge } \\
\text { Units }\end{array}$} & \multirow{2}{*}{$\begin{array}{l}\text { Pump } \\
\text { Intake } \\
\text { Position } \\
\text { (ft BGL) }\end{array}$} & \multirow{2}{*}{$\begin{array}{l}\text { Temperature } \\
\left({ }^{\circ} \mathrm{C}\right)\end{array}$} & \multirow[b]{2}{*}{$\mathrm{pH}$} & \multirow{2}{*}{$\begin{array}{c}\text { Conductivity } \\
(\mu \mathrm{S} / \mathrm{cm})\end{array}$} & \multirow{2}{*}{$\begin{array}{c}\text { Dissolved } \\
\text { Oxygen } \\
\text { (mg/L) }\end{array}$} & \multirow{2}{*}{$\begin{array}{l}\text { ORP } \\
(\mathrm{mV})\end{array}$} & \multirow{2}{*}{$\begin{array}{l}\text { Iron II } \\
\text { (mg/L) }\end{array}$} & \multirow{2}{*}{$\begin{array}{l}\text { Carbon } \\
\text { Dioxide } \\
(\mathrm{mg} / \mathrm{L})\end{array}$} & \multirow[b]{2}{*}{ Sample } \\
\hline & & & Water & Well & & & & & & & & & & & \\
\hline MW4S & $17-47$ & $9 / 22 / 10$ & 33.2 & 47.8 & 6.0 & $\mathrm{~L}$ & 32.0 & 16.3 & 7.07 & 732 & 7.28 & 90 & 0 & - & MRMW4S-W-30014 \\
\hline MW4S & $17-47$ & $4 / 21 / 11$ & 42.4 & 47.8 & 5.0 & $\mathrm{~L}$ & 44.7 & 12.6 & 7.15 & 595 & 7.25 & 138 & 0 & - & MRMW4S-W-30042 \\
\hline MW4S & $17-47$ & $10 / 4 / 11$ & 35.6 & 47.8 & 6.0 & L & 40.9 & 16.0 & 7.95 & 606 & 7.62 & 115 & 0 & - & MRMW4S-W-30071 \\
\hline MW5S & $15-55$ & $10 / 22 / 03$ & 31.4 & 55.7 & 48 & gal & - & 15.3 & 7.10 & 816 & - & 6 & - & - & MRMW05-W-16420 \\
\hline MW5S & $15-55$ & $6 / 2 / 04$ & 26.3 & 55.7 & $>57$ & gal & - & 14.3 & 7.21 & 817 & - & - & - & - & MRMW5S-W-16460 \\
\hline MW5S & $15-55$ & 9/13/05 & 22.7 & 54.2 & 75 & gal & - & 16.0 & 7.04 & 763 & 13.90 & 228 & 0 & 60 & MRMW5S-W-19260 \\
\hline MW5S & $15-55$ & $3 / 22 / 06$ & 28.6 & 54.5 & 50 & gal & - & 13.9 & 7.25 & 781 & 4.52 & 234 & 0.06 & 35 & MRMW5S-W-19996 \\
\hline MW5S & $15-55$ & 9/20/06 & 25.4 & 54.6 & 52 & gal & - & 13.9 & 7.19 & 787 & 5.82 & 73 & 0 & 35 & MRMW5S-W-22493 \\
\hline MW5S & $15-55$ & $3 / 22 / 07$ & 25.1 & 54.6 & 58 & gal & - & 15.5 & 6.50 & 436 & 3.98 & 159 & 0.08 & 30 & MRMW5S-W-16569 \\
\hline MW5S & $15-55$ & $10 / 3 / 07$ & 19.6 & 54.7 & 68 & gal & - & 16.5 & 7.18 & 850 & 1.87 & 268 & 0.04 & 25 & MRMW5S-W-16588 \\
\hline MW5S & $15-55$ & $4 / 14 / 08$ & 11.2 & 54.6 & 6.0 & $\mathrm{~L}$ & - & 14.1 & 6.90 & 1008 & 3.73 & 143 & 0.02 & - & MRMW5S-W-23235 \\
\hline MW5S & $15-55$ & $4 / 23 / 08$ & 11.3 & 54.6 & 6.5 & L & - & 14.9 & 6.88 & 1009 & 3.27 & 184 & - & - & MRMW5S-W-23266 \\
\hline MW5S & $15-55$ & $5 / 1 / 08$ & - & - & 3.7 & $\mathrm{~L}$ & 20.0 & 15.2 & 6.92 & 1014 & 2.99 & 126 & - & - & MRMW5S-20-W-23272 \\
\hline MW5S & $15-55$ & $5 / 1 / 08$ & - & - & 3.4 & $\mathrm{~L}$ & 28.0 & 15.1 & 6.90 & 997 & 2.38 & 124 & - & - & MRMW5S-28-W-23273 \\
\hline MW5S & $15-55$ & $5 / 1 / 08$ & - & - & 4.0 & L & 52.0 & 15.1 & 6.89 & 989 & 2.06 & 128 & - & - & MRMW5S-52-W-23274 \\
\hline MW5S & $15-55$ & $10 / 21 / 08$ & 22.5 & 54.6 & 7.0 & $\mathrm{~L}$ & 35.0 & 13.2 & 7.04 & 818 & 5.54 & 180 & 0 & - & MRMW5S-W-27625 \\
\hline MW5S & $15-55$ & $4 / 24 / 09$ & 22.1 & 54.6 & 5.5 & $\mathrm{~L}$ & 38.4 & 15.2 & 6.98 & 817 & 6.68 & 77 & 0 & - & MRMW5S-W-27657 \\
\hline MW5S & $15-55$ & 9/3/09 & 17.6 & 54.6 & 5.5 & $\mathrm{~L}$ & 36.3 & 16.5 & 7.10 & 873 & 3.07 & 23 & 0.02 & - & MRMW5S-W-29947 \\
\hline MW5S & $15-55$ & $4 / 7 / 10$ & 8.3 & 54.5 & 5.5 & $\mathrm{~L}$ & 35.0 & 12.6 & 6.76 & 844 & 4.46 & 149 & 0 & - & MRMW5S-W-29987 \\
\hline MW5S & $15-55$ & $9 / 22 / 10$ & 19.3 & 55.0 & 6.5 & L & 35.0 & 16.4 & 6.81 & 891 & 1.69 & 112 & 0 & - & MRMW5S-W-30015 \\
\hline MW5S & $15-55$ & $4 / 20 / 11$ & 24.4 & 54.6 & 7.0 & $\mathrm{~L}$ & 36.0 & 14.6 & 7.17 & 617 & 5.34 & 89 & 0 & - & MRMW5S-W-30043 \\
\hline MW5S & $15-55$ & $10 / 4 / 11$ & 21.1 & 54.6 & 6.5 & $\mathrm{~L}$ & 38.3 & 16.7 & 7.72 & 740 & 4.37 & 118 & 0.03 & - & MRMW5S-W-30072 \\
\hline MW6S & $10-25$ & $6 / 3 / 04$ & 3.3 & 26.9 & 45 & gal & - & 15.1 & 6.89 & 2410 & - & - & - & - & MRMW6S-W-16465 \\
\hline MW6S & $10-25$ & 9/14/05 & 4.7 & 26.9 & 43 & gal & - & 14.1 & 7.06 & 2350 & 0.01 & 54 & 0 & 60 & MRMW6S-W-19263 \\
\hline MW6S & $10-25$ & $3 / 20 / 06$ & 5.4 & 26.9 & 43 & gal & - & 9.8 & 6.91 & 2360 & 1.37 & 89 & 0.38 & 60 & MRMW6S-W-19990 \\
\hline MW6S & $10-25$ & 9/18/06 & 5.5 & 26.9 & 27 & gal & - & 12.5 & 6.96 & 2410 & 0.08 & -29 & 0.35 & 85 & MRMW6S-W-22486 \\
\hline MW6S & $10-25$ & $3 / 21 / 07$ & 5.4 & 26.9 & 30 & gal & - & 18.0 & 6.34 & 2450 & 0.12 & 75 & 0.78 & 40 & MRMW6S-W-16486 \\
\hline MW6S & $10-25$ & $10 / 2 / 07$ & 5.0 & 26.9 & 31 & gal & - & 17.1 & 7.33 & 2280 & 0.20 & 61 & 0.19 & 35 & MRMW6S-W-16583 \\
\hline MW6S & $10-25$ & $4 / 15 / 08$ & 5.2 & 26.9 & 2.5 & $\mathrm{~L}$ & - & 8.7 & 6.99 & 2485 & 0.31 & -76 & 0.41 & - & MRMW6S-W-23236 \\
\hline MW6S & $10-25$ & $10 / 20 / 08$ & 5.7 & 26.9 & 5.0 & $\mathrm{~L}$ & 17.5 & 14.3 & 6.84 & 2380 & 0.36 & 18 & 0.28 & - & MRMW6S-W-27626 \\
\hline MW6S & $10-25$ & $4 / 24 / 09$ & 6.2 & 26.9 & 12 & $\mathrm{~L}$ & 17.5 & 15.2 & 6.93 & 2270 & 0.19 & -39 & 0.63 & - & MRMW6S-W-27658 \\
\hline MW6S & $10-25$ & $9 / 4 / 09$ & 5.9 & 26.9 & 5.4 & $\mathrm{~L}$ & 17.5 & 13.8 & 6.88 & 2302 & 0.64 & 79 & 0.32 & - & MRMW6S-W-29948 \\
\hline MW6S & $10-25$ & $4 / 6 / 10$ & 6.2 & 26.9 & 8.0 & L & 17.5 & 15.2 & 6.87 & 2141 & 0.05 & -19 & 0.21 & - & MRMW6S-W-29988 \\
\hline MW6S & $10-25$ & $9 / 22 / 10$ & 5.5 & 26.9 & 8.8 & $\mathrm{~L}$ & 17.5 & 15.6 & 6.92 & 2354 & 0.42 & 47 & 0.24 & - & MRMW6S-W-30016 \\
\hline MW6S & $10-25$ & $4 / 20 / 11$ & 6.5 & 24.9 & 8.0 & $\mathrm{~L}$ & 17.5 & 13.0 & 6.73 & 1867 & 0.18 & 28 & 0.09 & - & MRMW6S-W-30044 \\
\hline MW6S & $10-25$ & 10/4/11 & 6.5 & 26.9 & 6.0 & $\mathrm{~L}$ & 17.5 & 16.6 & 7.11 & 2020 & 0.14 & 14 & 0.34 & - & MRMW6S-W-30073 \\
\hline MW7S & $20-45$ & $6 / 3 / 04$ & 26.7 & 47.0 & 40 & gal & - & 13.8 & 7.19 & 763 & - & - & - & - & MRMW7S-W-16466 \\
\hline MW7S & $20-45$ & 9/12/05 & 17.6 & 46.9 & 55 & gal & - & 15.0 & 7.26 & 760 & 8.35 & 240 & 0 & 50 & MRMW7S-W-19258 \\
\hline MW7S & $20-45$ & $3 / 22 / 06$ & 22.5 & 47.0 & 48 & gal & - & 15.2 & 7.32 & 740 & 5.52 & 268 & 0.03 & 25 & MRMW7S-W-20000 \\
\hline MW7S & $20-45$ & 9/19/06 & 20.9 & 47.0 & 56 & gal & - & 13.2 & 7.15 & 764 & 7.37 & 114 & 0 & 25 & MRMW7S-W-22490 \\
\hline MW7S & $20-45$ & $3 / 20 / 07$ & 18.0 & 47.0 & 50 & gal & - & 14.6 & 6.43 & 750 & 5.31 & 95 & 0 & 30 & MRMW7S-W-16481 \\
\hline MW7S & $20-45$ & $10 / 1 / 07$ & 12.4 & 47.0 & 70 & gal & - & 15.6 & 6.99 & 725 & 7.76 & 269 & 0.01 & 35 & MRMW7S-W-16581 \\
\hline MW7S & $20-45$ & $4 / 14 / 08$ & 7.7 & 47.0 & 1.8 & $\mathrm{~L}$ & - & 13.4 & 7.21 & 811 & 2.50 & 276 & 0 & - & MRMW7S-W-23237 \\
\hline MW7S & $20-45$ & $4 / 23 / 08$ & 7.8 & 47.0 & 11 & $\mathrm{~L}$ & - & 14.0 & 7.00 & 822 & 7.41 & 191 & - & - & MRMW7S-W-23265 \\
\hline MW7S & $20-45$ & $10 / 20 / 08$ & 17.2 & 47.0 & 6.3 & $\mathrm{~L}$ & 32.5 & 14.9 & 7.02 & 802 & 6.38 & 87 & 0 & - & MRMW7S-W-27627 \\
\hline
\end{tabular}


TABLE 3.3 (Cont.)

\begin{tabular}{|c|c|c|c|c|c|c|c|c|c|c|c|c|c|c|c|}
\hline \multirow[b]{2}{*}{ Location } & \multirow{2}{*}{$\begin{array}{l}\text { Screen } \\
\text { Interval } \\
\text { (ft BGL) }\end{array}$} & \multirow{2}{*}{$\begin{array}{l}\text { Sample } \\
\text { Date }\end{array}$} & \multicolumn{2}{|c|}{ Depth (ft BTOC) } & \multirow{2}{*}{$\begin{array}{l}\text { Volume } \\
\text { Purged }\end{array}$} & \multirow{2}{*}{$\begin{array}{l}\text { Purge } \\
\text { Units }\end{array}$} & \multirow{2}{*}{$\begin{array}{l}\text { Pump } \\
\text { Intake } \\
\text { Position } \\
\text { (ft BGL) }\end{array}$} & \multirow{2}{*}{$\begin{array}{l}\text { Temperature } \\
\left({ }^{\circ} \mathrm{C}\right)\end{array}$} & \multirow[b]{2}{*}{$\mathrm{pH}$} & \multirow{2}{*}{$\begin{array}{c}\text { Conductivity } \\
(\mu \mathrm{S} / \mathrm{cm})\end{array}$} & \multirow{2}{*}{$\begin{array}{c}\text { Dissolved } \\
\text { Oxygen } \\
\text { (mg/L) }\end{array}$} & \multirow{2}{*}{$\begin{array}{l}\text { ORP } \\
(\mathrm{mV})\end{array}$} & \multirow{2}{*}{$\begin{array}{l}\text { Iron II } \\
(\mathrm{mg} / \mathrm{L})\end{array}$} & \multirow{2}{*}{$\begin{array}{l}\text { Carbon } \\
\text { Dioxide } \\
\text { (mg/L) }\end{array}$} & \multirow[b]{2}{*}{ Sample } \\
\hline & & & Water & Well & & & & & & & & & & & \\
\hline MW7S & $20-45$ & $4 / 23 / 09$ & 16.7 & 47.0 & 7.0 & $\mathrm{~L}$ & 32.5 & 16.1 & 7.01 & 727 & 9.48 & -53 & 0.01 & - & MRMW7S-W-27659 \\
\hline MW7S & $20-45$ & 9/3/09 & 13.8 & 47.0 & 9.0 & $\mathrm{~L}$ & 32.5 & 17.4 & 7.58 & 814 & 9.86 & 102 & 0.02 & - & MRMW7S-W-29949 \\
\hline MW7S & $20-45$ & $4 / 6 / 10$ & 6.4 & 47.0 & 5.4 & $\mathrm{~L}$ & 32.5 & 14.7 & 7.04 & 718 & 7.92 & 23 & 0 & - & MRMW7S-W-29989 \\
\hline MW7S & $20-45$ & 9/23/10 & 14.2 & 45.0 & 6.0 & $\mathrm{~L}$ & 32.5 & 16.0 & 6.91 & 772 & 8.39 & 201 & 0 & - & MRMW7S-W-30017 \\
\hline MW7S & $20-45$ & $4 / 20 / 11$ & 19.7 & 46.7 & 6.0 & L & 32.5 & 13.0 & 7.00 & 598 & 7.91 & 128 & 0 & - & MRMW7S-W-30045 \\
\hline MW7S & $20-45$ & $10 / 4 / 11$ & 7.1 & 47.0 & 7.0 & L & 32.5 & 16.2 & 7.18 & 674 & 8.17 & 205 & 0.01 & - & MRMW7S-W-30074 \\
\hline MW8S & $10-25$ & $6 / 3 / 04$ & 3.7 & 26.8 & 45 & gal & - & 12.8 & 7.12 & 941 & - & - & - & - & MRMW8S-W-16464 \\
\hline MW8S & $10-25$ & 9/14/05 & 4.0 & 26.8 & 57 & gal & - & 14.1 & 7.30 & 853 & 0.02 & 65 & 0 & 40 & MRMW8S-W-19265 \\
\hline MW8S & $10-25$ & $3 / 20 / 06$ & 4.6 & 26.4 & 43 & gal & - & 12.5 & 7.04 & 954 & 0.90 & 153 & 0.05 & 30 & MRMW8S-W-19991 \\
\hline MW8S & $10-25$ & 9/19/06 & 4.8 & 26.8 & 45 & gal & - & 11.8 & 7.09 & 903 & 0.58 & 284 & 0.13 & 50 & MRMW8S-W-22492 \\
\hline MW8S & $10-25$ & $3 / 20 / 07$ & 2.6 & 26.8 & 49 & gal & - & 11.0 & 6.52 & 1026 & 0.77 & 76 & 0 & 30 & MRMW8S-W-16483 \\
\hline MW8S & $10-25$ & $10 / 2 / 07$ & 2.2 & 26.8 & 48 & gal & - & 15.2 & 6.76 & 607 & 2.66 & 209 & 0.02 & 25 & MRMW8S-W-16584 \\
\hline MW8S & $10-25$ & $4 / 15 / 08$ & 0.7 & 26.8 & 5.5 & $\mathrm{~L}$ & - & 10.2 & 7.27 & 1067 & 1.58 & 170 & 0 & - & MRMW8S-W-23238 \\
\hline MW8S & $10-25$ & $10 / 20 / 08$ & 3.6 & 26.8 & 8.0 & L & 17.5 & 14.0 & 6.91 & 1002 & 0.93 & 69 & 0 & - & MRMW8S-W-27628 \\
\hline MW8S & $10-25$ & $4 / 23 / 09$ & 2.3 & 26.8 & 6.0 & $\mathrm{~L}$ & 17.5 & 11.4 & 6.88 & 825 & 1.76 & -35 & 0.02 & - & MRMW8S-W-27660 \\
\hline MW8S & $10-25$ & 9/3/09 & 2.9 & 26.8 & 8.5 & $\mathrm{~L}$ & 17.5 & 14.1 & 7.52 & 890 & 2.09 & 115 & 0 & - & MRMW8S-W-29950 \\
\hline MW8S & $10-25$ & $4 / 6 / 10$ & 1.1 & 26.8 & 8.0 & L & 17.5 & 13.7 & 7.00 & 843 & 2.60 & 212 & 0 & - & MRMW8S-W-29990 \\
\hline MW8S & $10-25$ & $9 / 22 / 10$ & 2.5 & 26.8 & 9.0 & L & 17.5 & 16.2 & 7.12 & 865 & 1.51 & 100 & 0.04 & - & MRMW8S-W-30018 \\
\hline MW8S & $10-25$ & $4 / 20 / 11$ & 2.7 & 26.8 & 9.0 & $\mathrm{~L}$ & 17.5 & 10.1 & 6.88 & 652 & 0.3 & 98 & 0 & - & MRMW8S-W-30046 \\
\hline MW8S & $10-25$ & 10/4/11 & 4.9 & 26.3 & 9.0 & $\mathrm{~L}$ & 17.5 & 14.4 & 7.16 & 711 & 1.22 & 191 & 0.04 & - & MRMW8S-W-30075 \\
\hline MW9S & $38.83-53.83$ & $3 / 22 / 06$ & 20.2 & 58.6 & 20 & gal & - & 14.6 & 7.17 & 715 & 0.41 & 25 & 0 & 35 & MRMW9S-W-20004 \\
\hline MW9S & $38.83-53.83$ & 9/19/06 & 18.9 & 59.0 & 22 & gal & - & 13.0 & 7.08 & 707 & 0.10 & 113 & 0 & 55 & MRMW9S-W-22494 \\
\hline MW9S & $38.83-53.83$ & $3 / 20 / 07$ & 16.7 & 59.0 & 22 & gal & - & 14.2 & 6.39 & 714 & 0.21 & 40 & 0 & 20 & MRMW9S-W-16480 \\
\hline MW9S & $38.83-53.83$ & $10 / 1 / 07$ & 14.0 & 58.6 & 23 & gal & - & 15.5 & 7.05 & 664 & 5.50 & 191 & 0 & 30 & MRMW9S-W-16582 \\
\hline MW9S & $38.83-53.83$ & $4 / 14 / 08$ & 16.6 & 58.6 & 2.3 & L & - & 12.6 & 7.33 & 709 & 1.93 & 266 & 0.07 & - & MRMW9S-W-23239 \\
\hline MW9S & $38.83-53.83$ & $10 / 20 / 08$ & 21.5 & 58.5 & 11 & $\mathrm{~L}$ & 46.3 & 15.1 & 7.15 & 690 & 6.18 & 106 & 0 & - & MRMW9S-W-27629 \\
\hline MW9S & $38.83-53.83$ & $4 / 23 / 09$ & 21.9 & 58.5 & 5.5 & L & 46.3 & 16.0 & 7.17 & 669 & 5.99 & -65 & 0.07 & - & MRMW9S-W-27661 \\
\hline MW9S & $38.83-53.83$ & 9/4/09 & 20.0 & 58.5 & 5.0 & L & 46.3 & 17.3 & 7.18 & 684 & 5.33 & 52 & 0.03 & - & MRMW9S-W-29951 \\
\hline MW9S & $38.83-53.83$ & $4 / 6 / 10$ & 16.5 & 58.5 & 6.0 & L & 46.3 & 15.3 & 7.19 & 650 & 5.50 & -10 & 0.02 & - & MRMW9S-W-29991 \\
\hline MW9S & $38.83-53.83$ & $9 / 22 / 10$ & 21.2 & 53.8 & 7.0 & L & 46.3 & 17.1 & 7.12 & 704 & 6.07 & 151 & 0 & - & MRMW9S-W-30019 \\
\hline MW9S & $38.83-53.83$ & $4 / 20 / 11$ & 23.8 & 58.3 & 14 & L & 46.3 & 13.6 & 7.07 & 569 & 5.42 & 111 & 0 & - & MRMW9S-W-30047 \\
\hline MW9S & $38.83-53.83$ & $10 / 4 / 11$ & 22.4 & 58.5 & 8.0 & $\mathrm{~L}$ & 46.3 & 16.7 & 7.14 & 618 & 5.9 & 185 & 0.05 & - & MRMW9S-W-30076 \\
\hline MW10S & $30-45$ & $3 / 21 / 06$ & 12.3 & 49.6 & 19 & gal & - & 6.3 & 7.11 & 701 & 2.10 & 88 & 0.01 & 40 & MRMW10S-W-19999 \\
\hline MW10S & $30-45$ & $9 / 18 / 06$ & 11.1 & 49.6 & 20 & gal & - & 14.3 & 7.17 & 701 & 0.04 & 24 & 0.08 & 60 & MRMW10S-W-22489 \\
\hline MW10S & $30-45$ & $3 / 21 / 07$ & 10.8 & 49.6 & 20 & gal & - & 14.5 & 6.51 & 720 & 0.88 & 11 & 0 & 30 & MRMW10S-W-16485 \\
\hline MW10S & $30-45$ & $10 / 1 / 07$ & 7.0 & 49.7 & 20 & gal & - & 16.3 & 6.97 & 664 & 0.35 & 248 & 0.04 & 35 & MRMW10S-W-16593 \\
\hline MW10S & $30-45$ & $4 / 14 / 08$ & 9.8 & 49.7 & 1.9 & $\mathrm{~L}$ & - & 16.0 & 7.25 & 723 & 1.25 & 181 & 0 & - & MRMW10S-W-23240 \\
\hline MW10S & $30-45$ & $10 / 20 / 08$ & 13.7 & 49.7 & 5.4 & L & 37.5 & 14.3 & 7.03 & 710 & 1.02 & 56 & 0 & - & MRMW10S-W-27630 \\
\hline MW10S & $30-45$ & $4 / 23 / 09$ & 13.6 & 45.0 & 7.5 & L & 37.5 & 15.1 & 7.05 & 668 & 1.78 & -57 & 0.07 & - & MRMW10S-W-27662 \\
\hline MW10S & $30-45$ & 9/3/09 & 12.0 & 49.7 & 7.5 & $\mathrm{~L}$ & 37.5 & 14.3 & 7.59 & 731 & 2.03 & 86 & 0.07 & - & MRMW10S-W-29952 \\
\hline MW10S & $30-45$ & $4 / 6 / 10$ & 7.5 & 49.7 & 9.0 & $\mathrm{~L}$ & 37.5 & 16.1 & 7.06 & 649 & 2.91 & 221 & 0 & - & MRMW10S-W-29992 \\
\hline MW10S & $30-45$ & $9 / 22 / 10$ & 11.7 & 49.7 & 11 & L & 37.5 & 16.7 & 7.29 & 723 & 5.05 & 131 & 0 & - & MRMW10S-W-30020 \\
\hline MW10S & $30-45$ & $4 / 20 / 11$ & 13.7 & 49.3 & 7.3 & L & 37.5 & 12.8 & 7.06 & 573 & 2.09 & 99 & 0.1 & - & MRMW10S-W-30048 \\
\hline MW10S & $30-45$ & $10 / 4 / 11$ & 12.5 & 49.7 & 8.0 & $\mathrm{~L}$ & 37.5 & 15.4 & 7.27 & 613 & 2.53 & 201 & 0.01 & - & MRMW10S-W-30077 \\
\hline
\end{tabular}


TABLE 3.3 (Cont.) 
TABLE 3.3 (Cont.)

\begin{tabular}{|c|c|c|c|c|c|c|c|c|c|c|c|c|c|c|c|}
\hline \multirow[b]{2}{*}{ Location } & \multirow{2}{*}{$\begin{array}{l}\text { Screen } \\
\text { Interval } \\
\text { (ft BGL) }\end{array}$} & \multirow{2}{*}{$\begin{array}{l}\text { Sample } \\
\text { Date }\end{array}$} & \multicolumn{2}{|c|}{ Depth (ft BTOC) } & \multirow{2}{*}{$\begin{array}{l}\text { Volume } \\
\text { Purged }\end{array}$} & \multirow{2}{*}{$\begin{array}{l}\text { Purge } \\
\text { Units }\end{array}$} & \multirow{2}{*}{$\begin{array}{l}\text { Pump } \\
\text { Intake } \\
\text { Position } \\
\text { (ft BGL) }\end{array}$} & \multirow{2}{*}{$\begin{array}{l}\text { Temperature } \\
\left({ }^{\circ} \mathrm{C}\right)\end{array}$} & \multirow[b]{2}{*}{$\mathrm{pH}$} & \multirow{2}{*}{$\begin{array}{l}\text { Conductivity } \\
(\mu \mathrm{S} / \mathrm{cm})\end{array}$} & \multirow{2}{*}{$\begin{array}{l}\text { Dissolved } \\
\text { Oxygen } \\
\text { (mg/L) }\end{array}$} & \multirow{2}{*}{$\begin{array}{l}\text { ORP } \\
(\mathrm{mV})\end{array}$} & \multirow{2}{*}{$\begin{array}{l}\text { Iron II } \\
\text { (mg/L) }\end{array}$} & \multirow{2}{*}{$\begin{array}{l}\text { Carbon } \\
\text { Dioxide } \\
\text { (mg/L) }\end{array}$} & \multirow[b]{2}{*}{ Sample } \\
\hline & & & Water & Well & & & & & & & & & & & \\
\hline Stone & 43 & $3 / 19 / 06$ & 17.4 & 40.0 & 100 & gal & - & 12.9 & 6.42 & 650 & - & 213 & 0 & - & MRSTONE-W-19987 \\
\hline Stone & 43 & 9/19/06 & 18.6 & 38.8 & 41 & gal & - & 16.7 & 7.12 & 639 & - & - & - & - & MRSTONE-W-16529 \\
\hline Stone & 43 & $3 / 22 / 07$ & 20.6 & 38.8 & 56 & gal & - & 16.7 & 6.58 & 679 & 4.71 & 19 & 0.28 & 35 & MRSTONE-W-16560 \\
\hline Stone & 43 & $10 / 3 / 07$ & 14.6 & 38.6 & 72 & gal & - & 16.1 & 6.97 & 564 & 7.07 & 225 & 0.07 & 25 & MRSTONE-W-16589 \\
\hline Stone & 43 & $4 / 15 / 08$ & - & 38.9 & - & - & - & 11.3 & 7.45 & 557 & - & - & 0 & - & MRSTONE-W-23244 \\
\hline Stone & 43 & $10 / 21 / 08$ & - & & 5.0 & gal & - & - & - & - & - & - & - & - & MRSTONE-W-27634 \\
\hline Stone & 43 & $4 / 23 / 09$ & - & - & 5.0 & gal & - & 13.9 & 7.12 & 588 & - & - & - & - & MRSTONE-W-27666 \\
\hline Stone & 43 & $9 / 2 / 09$ & - & - & 5.0 & gal & - & 13.8 & 7.40 & 623 & - & - & - & - & MRSTONE-W-29956 \\
\hline Stone & 43 & $4 / 7 / 10$ & - & - & 5.0 & gal & - & 10.9 & 6.83 & 468 & - & - & - & - & MRSTONE-W-29996 \\
\hline Stone & 43 & 9/22/10 & - & - & 5.0 & gal & - & 15.3 & 7.21 & 552 & - & - & - & - & MRSTONE-W-30024 \\
\hline Stone & 43 & $4 / 21 / 11$ & - & - & 5.0 & gal & - & 12.5 & 7.52 & 455 & - & - & - & - & MRSTONE-W-30052 \\
\hline Stone & 43 & $10 / 3 / 11$ & - & - & 5.0 & gal & - & 14.1 & 7.38 & 495 & - & - & 0.04 & - & MRStone-W-30081 \\
\hline TD12 & $27-67$ & $4 / 20 / 11$ & - & - & - & - & - & - & - & - & - & - & - & - & MRTD12-W-30053 \\
\hline TD12 & $27-67$ & 10/3/11 & - & - & - & - & - & 14.2 & 7.46 & 2083 & - & - & - & - & MRTD12-W-30082 \\
\hline
\end{tabular}

a Not measured.

b TWV, three well volumes.

c PDS, purged dry and then sampled.

d The well's dedicated pump was used for sampling. The pump was allowed to run before the sample was collected.

e Total depth. 
TABLE 3.4 Results of analyses at the AGEM Laboratory for volatile organic compounds in surface water and sediment samples collected in 2007-2011. ${ }^{\mathrm{a}}$

\begin{tabular}{|c|c|c|c|c|c|c|c|}
\hline \multirow[b]{2}{*}{ Location } & \multirow[b]{2}{*}{ Sample } & \multirow[b]{2}{*}{$\begin{array}{c}\text { Sample } \\
\text { Date }\end{array}$} & \multirow[b]{2}{*}{ Medium } & \multicolumn{4}{|c|}{ Concentration ( $\mu \mathrm{g} / \mathrm{L}$ in water; $\mu \mathrm{g} / \mathrm{kg}$ in sediment) } \\
\hline & & & & $\begin{array}{c}\text { Carbon } \\
\text { Tetrachloride }\end{array}$ & Chloroform & $\begin{array}{l}\text { Methylene } \\
\text { Chloride }\end{array}$ & $\begin{array}{c}\text { Quantitation } \\
\text { Limit }\end{array}$ \\
\hline SM1 & MRSM1-W-16572 & $3 / 22 / 07$ & Water & $N D^{b}$ & ND & ND & 1 \\
\hline SM1 & MRSM1-S-16573 & $3 / 22 / 07$ & Sediment & ND & ND & ND & 10 \\
\hline SM1 & MRSM1-W-16583 & $10 / 8 / 07$ & Water & ND & ND & ND & 1 \\
\hline SM1 & MRSM1-S-16584 & $10 / 8 / 07$ & Sediment & ND & ND & ND & 10 \\
\hline SM1 & MRSM1-W-23254 & 4/14/08 & Water & ND & ND & ND & 1 \\
\hline SM1 & MRSM1-S-23254 & $4 / 14 / 08$ & Sediment & ND & ND & ND & 10 \\
\hline SM1 & MRSM1-W-27644 & 10/20/08 & Water & ND & ND & ND & 1 \\
\hline SM1 & MRSM1-S-27644 & 10/20/08 & Sediment & ND & ND & ND & 10 \\
\hline SM1 & MRSM1-W-27676 & 4/22/09 & Water & ND & ND & ND & 1 \\
\hline SM1 & MRSM1-S-27676 & $4 / 22 / 09$ & Sediment & ND & ND & ND & 10 \\
\hline SM1 & MRSM1-W-29966 & 9/2/09 & Water & ND & ND & ND & 1 \\
\hline SM1 & MRSM1-S-29966 & $9 / 2 / 09$ & Sediment & ND & ND & ND & 10 \\
\hline SM1 & MRSM1-W-29974 & $4 / 6 / 10$ & Water & ND & ND & ND & 1 \\
\hline SM1 & MRSM1-S-29974 & $4 / 6 / 10$ & Sediment & ND & ND & ND & 10 \\
\hline SM1 & MRSM1-W-30005 & $9 / 22 / 10$ & Water & ND & ND & ND & 1 \\
\hline SM1 & MRSM1-S-30005 & $9 / 22 / 10$ & Sediment & ND & ND & ND & 10 \\
\hline SM1 & MRSM1-W-29974 & $4 / 6 / 10$ & Water & ND & ND & ND & 1 \\
\hline SM1 & MRSM1-S-29974 & $4 / 6 / 10$ & Sediment & ND & ND & ND & 10 \\
\hline SM1 & MRSM1-W-30062 & $10 / 3 / 11$ & Water & ND & ND & ND & 1 \\
\hline SM1 & MRSM1-S-30062 & $10 / 3 / 11$ & Sediment & ND & ND & ND & 10 \\
\hline SM2 & MRSM2-W-16574 & $3 / 22 / 07$ & Water & ND & ND & ND & 1 \\
\hline SM2 & MRSM2-S-16575 & $3 / 22 / 07$ & Sediment & ND & ND & ND & 10 \\
\hline SM2 & MRSM2-W-16585 & 10/8/07 & Water & ND & ND & ND & 1 \\
\hline SM2 & MRSM2-S-16586 & 10/8/07 & Sediment & ND & ND & ND & 10 \\
\hline SM2 & MRSM2-W-23255 & $4 / 14 / 08$ & Water & ND & ND & ND & 1 \\
\hline SM2 & MRSM2-S-23255 & 4/14/08 & Sediment & ND & ND & ND & 10 \\
\hline SM2 & MRSM2-W-27645 & 10/20/08 & Water & ND & ND & ND & 1 \\
\hline SM2 & MRSM2-S-27645 & 10/20/08 & Sediment & ND & ND & ND & 10 \\
\hline SM2 & MRSM2-W-27677 & $4 / 22 / 09$ & Water & ND & ND & ND & 1 \\
\hline SM2 & MRSM2-S-27677 & $4 / 22 / 09$ & Sediment & ND & ND & ND & 10 \\
\hline SM2 & MRSM2-W-29967 & 9/2/09 & Water & ND & ND & ND & 1 \\
\hline SM2 & MRSM2-S-29967 & $9 / 2 / 09$ & Sediment & ND & ND & ND & 10 \\
\hline SM2 & MRSM2-W-29975 & $4 / 6 / 10$ & Water & ND & ND & ND & 1 \\
\hline SM2 & MRSM2-S-29975 & $4 / 6 / 10$ & Sediment & ND & ND & ND & 10 \\
\hline SM2 & MRSM2-W-30006 & $9 / 22 / 10$ & Water & ND & ND & ND & 1 \\
\hline SM2 & MRSM2-S-30006 & $9 / 22 / 10$ & Sediment & ND & ND & ND & 10 \\
\hline SM2 & MRSM2-W-29975 & $4 / 6 / 10$ & Water & ND & ND & ND & 1 \\
\hline SM2 & MRSM2-S-29975 & $4 / 6 / 10$ & Sediment & ND & ND & ND & 10 \\
\hline $\mathrm{SM} 2$ & MRSM2-W-30063 & $10 / 3 / 11$ & Water & ND & ND & ND & 1 \\
\hline SM2 & MRSM2-S-30063 & $10 / 3 / 11$ & Sediment & ND & ND & ND & 10 \\
\hline SM3 & MRSM3-W-16576 & $3 / 22 / 07$ & Water & ND & ND & ND & 1 \\
\hline SM3 & MRSM3-S-16577 & $3 / 22 / 07$ & Sediment & ND & ND & ND & 10 \\
\hline SM3 & MRSM3-W-16587 & 10/8/07 & Water & ND & ND & ND & 1 \\
\hline SM3 & MRSM3-S-16588 & $10 / 8 / 07$ & Sediment & ND & ND & ND & 10 \\
\hline SM3 & MRSM3-W-23256 & $4 / 14 / 08$ & Water & ND & ND & ND & 1 \\
\hline SM3 & MRSM3-S-23256 & 4/14/08 & Sediment & ND & ND & ND & 10 \\
\hline SM3 & MRSM3-W-27646 & $10 / 20 / 08$ & Water & ND & ND & ND & 1 \\
\hline SM3 & MRSM3-S-27646 & 10/20/08 & Sediment & ND & ND & ND & 10 \\
\hline SM3 & MRSM3-W-27678 & 4/22/09 & Water & ND & ND & ND & 1 \\
\hline SM3 & MRSM3-S-27678 & 4/22/09 & Sediment & ND & ND & ND & 10 \\
\hline
\end{tabular}


TABLE 3.4 (Cont.)

\begin{tabular}{|c|c|c|c|c|c|c|c|}
\hline \multirow[b]{2}{*}{ Location } & \multirow[b]{2}{*}{ Sample } & \multirow[b]{2}{*}{$\begin{array}{c}\text { Sample } \\
\text { Date }\end{array}$} & \multirow[b]{2}{*}{ Medium } & \multicolumn{4}{|c|}{ Concentration ( $\mu \mathrm{g} / \mathrm{L}$ in water; $\mu \mathrm{g} / \mathrm{kg}$ in sediment) } \\
\hline & & & & $\begin{array}{c}\text { Carbon } \\
\text { Tetrachloride }\end{array}$ & Chloroform & $\begin{array}{l}\text { Methylene } \\
\text { Chloride }\end{array}$ & $\begin{array}{l}\text { Quantitation } \\
\text { Limit }\end{array}$ \\
\hline SM3 & MRSM3-W-29968 & 9/2/09 & Water & ND & ND & ND & 1 \\
\hline SM3 & MRSM3-S-29968 & $9 / 2 / 09$ & Sediment & ND & ND & ND & 10 \\
\hline SM3 & MRSM3-W-29976 & $4 / 6 / 10$ & Water & ND & ND & ND & 1 \\
\hline SM3 & MRSM3-S-29976 & $4 / 6 / 10$ & Sediment & ND & ND & ND & 10 \\
\hline SM3 & MRSM3-W-30007 & $9 / 22 / 10$ & Water & ND & ND & ND & 1 \\
\hline SM3 & MRSM3-S-30007 & $9 / 22 / 10$ & Sediment & ND & ND & ND & 10 \\
\hline SM3 & MRSM3-W-29976 & $4 / 6 / 10$ & Water & ND & ND & ND & 1 \\
\hline SM3 & MRSM3-S-29976 & $4 / 6 / 10$ & Sediment & ND & ND & ND & 10 \\
\hline SM3 & MRSM3-W-30064 & $10 / 3 / 11$ & Water & ND & ND & ND & 1 \\
\hline SM3 & MRSM3-S-30064 & $10 / 3 / 11$ & Sediment & ND & ND & ND & 10 \\
\hline SM4 & MRSM4-W-16578 & $3 / 22 / 07$ & Water & ND & ND & ND & 1 \\
\hline SM4 & MRSM4-S-16579 & $3 / 22 / 07$ & Sediment & ND & ND & ND & 10 \\
\hline SM4 & MRSM4-W-16589 & $10 / 8 / 07$ & Water & ND & ND & ND & 1 \\
\hline SM4 & MRSM4-S-16590 & $10 / 8 / 07$ & Sediment & ND & ND & ND & 10 \\
\hline SM4 & MRSM4-W-23257 & $4 / 14 / 08$ & Water & ND & ND & ND & 1 \\
\hline SM4 & MRSM4-S-23257 & 4/14/08 & Sediment & ND & ND & ND & 10 \\
\hline SM4 & MRSM4-W-27647 & $10 / 20 / 08$ & Water & ND & ND & ND & 1 \\
\hline SM4 & MRSM4-S-27647 & $10 / 20 / 08$ & Sediment & ND & ND & ND & 10 \\
\hline SM4 & MRSM4-W-27679 & $4 / 22 / 09$ & Water & ND & ND & ND & 1 \\
\hline SM4 & MRSM4-S-27679 & $4 / 22 / 09$ & Sediment & ND & ND & ND & 10 \\
\hline SM4 & MRSM4-W-29969 & $9 / 2 / 09$ & Water & ND & ND & ND & 1 \\
\hline SM4 & MRSM4-S-29969 & $9 / 2 / 09$ & Sediment & ND & ND & ND & 10 \\
\hline SM4 & MRSM4-W-29977 & $4 / 6 / 10$ & Water & ND & ND & ND & 1 \\
\hline SM4 & MRSM4-S-29977 & $4 / 6 / 10$ & Sediment & ND & ND & ND & 10 \\
\hline SM4 & MRSM4-W-30008 & $9 / 22 / 10$ & Water & ND & ND & ND & 1 \\
\hline SM4 & MRSM4-S-30008 & $9 / 22 / 10$ & Sediment & ND & ND & ND & 10 \\
\hline SM4 & MRSM4-W-29977 & $4 / 6 / 10$ & Water & ND & ND & ND & 1 \\
\hline SM4 & MRSM4-S-29977 & $4 / 6 / 10$ & Sediment & ND & ND & ND & 10 \\
\hline SM4 & MRSM4-W-30065 & $10 / 3 / 11$ & Water & ND & ND & ND & 1 \\
\hline SM4 & MRSM4-S-30065 & $10 / 3 / 11$ & Sediment & ND & ND & ND & 10 \\
\hline SMB & MRSMB-W-16570 & $3 / 22 / 07$ & Water & ND & ND & ND & 1 \\
\hline SMB & MRSMB-S-16571 & $3 / 22 / 07$ & Sediment & ND & ND & ND & 10 \\
\hline SMB & MRSMB-W-16581 & $10 / 8 / 07$ & Water & ND & ND & ND & 1 \\
\hline SMB & MRSMB-S-16582 & $10 / 8 / 07$ & Sediment & ND & ND & ND & 10 \\
\hline SMB & MRSMB-W-23258 & $4 / 14 / 08$ & Water & ND & ND & ND & 1 \\
\hline SMB & MRSMB-S-23258 & $4 / 14 / 08$ & Sediment & ND & ND & ND & 10 \\
\hline SMB & MRSMB-W-27648 & $10 / 20 / 08$ & Water & ND & ND & ND & 1 \\
\hline SMB & MRSMB-S-27648 & $10 / 20 / 08$ & Sediment & ND & ND & ND & 10 \\
\hline SMB & MRSMB-W-27680 & $4 / 22 / 09$ & Water & ND & ND & ND & 1 \\
\hline SMB & MRSMB-S-27680 & $4 / 22 / 09$ & Sediment & ND & ND & ND & 10 \\
\hline SMB & MRSMB-W-29970 & $9 / 2 / 09$ & Water & ND & ND & ND & 1 \\
\hline SMB & MRSMB-S-29970 & $9 / 2 / 09$ & Sediment & ND & ND & ND & 10 \\
\hline SMB & MRSMB-W-29978 & $4 / 6 / 10$ & Water & ND & ND & ND & 1 \\
\hline SMB & MRSMB-S-29978 & $4 / 6 / 10$ & Sediment & ND & ND & ND & 10 \\
\hline SMB & MRSMB-W-30009 & $9 / 22 / 10$ & Water & ND & ND & ND & 1 \\
\hline SMB & MRSMB-S-30009 & $9 / 22 / 10$ & Sediment & ND & ND & ND & 10 \\
\hline SMB & MRSMB-W-29978 & $4 / 6 / 10$ & Water & ND & ND & ND & 1 \\
\hline SMB & MRSMB-S-29978 & $4 / 6 / 10$ & Sediment & ND & ND & ND & 10 \\
\hline SMB & MRSMB-W-30066 & $10 / 3 / 11$ & Water & ND & ND & ND & 1 \\
\hline SMB & MRSMB-S-30066 & $10 / 3 / 11$ & Sediment & ND & ND & ND & 10 \\
\hline
\end{tabular}


TABLE 3.4 (Cont.)

\begin{tabular}{|c|c|c|c|c|c|c|c|}
\hline \multirow[b]{2}{*}{ Location } & \multirow[b]{2}{*}{ Sample } & \multirow[b]{2}{*}{$\begin{array}{l}\text { Sample } \\
\text { Date }\end{array}$} & \multirow[b]{2}{*}{ Medium } & \multicolumn{4}{|c|}{ Concentration ( $\mu \mathrm{g} / \mathrm{L}$ in water; $\mu \mathrm{g} / \mathrm{kg}$ in sediment) } \\
\hline & & & & $\begin{array}{c}\text { Carbon } \\
\text { Tetrachloride }\end{array}$ & Chloroform & $\begin{array}{l}\text { Methylene } \\
\text { Chloride }\end{array}$ & $\begin{array}{l}\text { Quantitation } \\
\text { Limit }\end{array}$ \\
\hline
\end{tabular}

a Analyses conducted at the AGEM Laboratory by EPA Method 524.2 for surface water samples or by modified EPA Method 5030B/8260B for sediment samples.

b ND, not detected at the instrument detection limit of $0.1 \mu \mathrm{g} / \mathrm{L}$ for surface water samples or $1.0 \mu \mathrm{g} / \mathrm{kg}$ for sediment samples. 
TABLE 3.5 Results of analyses at the AGEM Laboratory for carbon tetrachloride and chloroform in vegetation samples collected in 2006-2011. ${ }^{\mathrm{a}}$

\begin{tabular}{|c|c|c|c|c|c|}
\hline \multirow[b]{2}{*}{ Location } & \multirow[b]{2}{*}{ Sample } & \multirow[b]{2}{*}{$\begin{array}{l}\text { Sample } \\
\text { Date }\end{array}$} & \multirow[b]{2}{*}{ Type } & \multicolumn{2}{|c|}{ Concentration $(\mu \mathrm{g} / \mathrm{kg})$} \\
\hline & & & & $\begin{array}{c}\text { Carbon } \\
\text { Tetrachloride }\end{array}$ & Chloroform \\
\hline MR001 & MR001-B-18959 & $10 / 14 / 06$ & Branch & $N D^{b}$ & 1.6 \\
\hline MR001 & MR001-L-18958 & $10 / 14 / 06$ & Leaf & ND & 3.3 \\
\hline MR001 & MR001-B-23173 & $4 / 2 / 07$ & Branch & ND & ND \\
\hline MR001 & MR001-B-23213 & $7 / 26 / 07$ & Branch & ND & ND \\
\hline MR001 & MR001-L-23212 & $7 / 26 / 07$ & Leaf & ND & ND \\
\hline MR001A & MR001A-B-16622 & $7 / 24 / 08$ & Branch & ND & ND \\
\hline MR001 & MR001-B-16623 & $7 / 24 / 08$ & Branch & ND & ND \\
\hline MR001 & MR001-B-29924 & $8 / 27 / 09$ & Branch & ND & ND \\
\hline MR001 & MR001-B-31929 & $7 / 27 / 10$ & Branch & ND & ND \\
\hline MR001 & MR001-B-29963 & $7 / 28 / 11$ & Branch & ND & ND \\
\hline MR002 & MR002-B-18961 & $10 / 14 / 06$ & Branch & ND & ND \\
\hline MR002 & MR002-L-18960 & $10 / 14 / 06$ & Leaf & ND & ND \\
\hline MR002 & MR002-B-23174 & $4 / 2 / 07$ & Branch & ND & 1.5 \\
\hline MR002 & MR002-B-23211 & $7 / 26 / 07$ & Branch & ND & ND \\
\hline MR002 & MR002-L-23210 & $7 / 26 / 07$ & Leaf & ND & 1.3 \\
\hline MR002 & MR002-B-16621 & $7 / 24 / 08$ & Branch & ND & ND \\
\hline MR002 & MR002-B-29923 & 8/27/09 & Branch & ND & ND \\
\hline MR002 & MR002-B-31928 & $7 / 27 / 10$ & Branch & ND & ND \\
\hline MR002 & MR002-B-29962 & $7 / 28 / 11$ & Branch & ND & 1.9 \\
\hline MR003 & MR003-B-18963 & $10 / 14 / 06$ & Branch & ND & 1.5 \\
\hline MR003 & MR003-L-18962 & $10 / 14 / 06$ & Leaf & ND & 1.8 \\
\hline MR003 & MR003-B-23175 & $4 / 2 / 07$ & Branch & ND & ND \\
\hline MR003 & MR003-B-23209 & $7 / 26 / 07$ & Branch & ND & ND \\
\hline MR003 & MR003-L-23208 & $7 / 26 / 07$ & Leaf & ND & 0.8 \\
\hline MR003 & MR003-B-16620 & $7 / 24 / 08$ & Branch & ND & ND \\
\hline MR003 & MR003-B-29922 & 8/27/09 & Branch & ND & ND \\
\hline MR003 & MR003-B-31927 & $7 / 27 / 10$ & Branch & ND & ND \\
\hline MR003 & MR003-B-29961 & $7 / 28 / 11$ & Branch & ND & ND \\
\hline MR004 & MR004-B-18965 & $10 / 14 / 06$ & Branch & ND & 2.1 \\
\hline MR004 & MR004-L-18964 & $10 / 14 / 06$ & Leaf & ND & ND \\
\hline MR004 & MR004-B-23176 & $4 / 2 / 07$ & Branch & ND & ND \\
\hline MR004 & MR004-B-23205 & $7 / 26 / 07$ & Branch & ND & ND \\
\hline MR004 & MR004-L-23204 & $7 / 26 / 07$ & Leaf & ND & ND \\
\hline MR004 & MR004-B-16619 & $7 / 24 / 08$ & Branch & ND & ND \\
\hline MR004 & MR004-B-29921 & $8 / 27 / 09$ & Branch & ND & ND \\
\hline MR004 & MR004-B-31926 & $7 / 27 / 10$ & Branch & ND & ND \\
\hline MR004 & MR004-B-29960 & $7 / 28 / 11$ & Branch & ND & ND \\
\hline MR005 & MR005-B-18967 & $10 / 14 / 06$ & Branch & ND & ND \\
\hline MR005 & MR005-L-18966 & $10 / 14 / 06$ & Leaf & ND & 1.2 \\
\hline MR005 & MR005-B-23177 & $4 / 2 / 07$ & Branch & ND & ND \\
\hline MR005 & MR005-B-23207 & $7 / 26 / 07$ & Branch & ND & ND \\
\hline MR005 & MR005-L-23206 & $7 / 26 / 07$ & Leaf & ND & 1.4 \\
\hline MR005A & MR005-B-16618 & $7 / 24 / 08$ & Branch & ND & ND \\
\hline MR005A & MR005A-B-29920 & $8 / 27 / 09$ & Branch & ND & ND \\
\hline MR005A & MR005A-B-31925 & $7 / 27 / 10$ & Branch & ND & ND \\
\hline MR005A & MR005A-B-29959 & $7 / 28 / 11$ & Branch & ND & ND \\
\hline
\end{tabular}


TABLE 3.5 (Cont.)

\begin{tabular}{|c|c|c|c|c|c|}
\hline \multirow[b]{2}{*}{ Location } & \multirow[b]{2}{*}{ Sample } & \multirow[b]{2}{*}{$\begin{array}{l}\text { Sample } \\
\text { Date }\end{array}$} & \multirow[b]{2}{*}{ Type } & \multicolumn{2}{|c|}{ Concentration $(\mu \mathrm{g} / \mathrm{kg})$} \\
\hline & & & & $\begin{array}{c}\text { Carbon } \\
\text { Tetrachloride }\end{array}$ & Chloroform \\
\hline MR006 & MR006-B-18969 & $10 / 14 / 06$ & Branch & ND & 3.8 \\
\hline MR006 & MR006-L-18968 & $10 / 14 / 06$ & Leaf & ND & ND \\
\hline MR006 & MR006-B-23161 & $4 / 2 / 07$ & Branch & ND & ND \\
\hline MR006 & MR006-B-23181 & $7 / 26 / 07$ & Branch & ND & ND \\
\hline MR006 & MR006-L-23180 & $7 / 26 / 07$ & Leaf & ND & 1.3 \\
\hline MR006 & MR006-B-16625 & $7 / 24 / 08$ & Branch & ND & ND \\
\hline MR006 & MR006-B-29930 & $8 / 27 / 09$ & Branch & ND & ND \\
\hline MR006 & MR006-B-31931 & $7 / 27 / 10$ & Branch & ND & ND \\
\hline MR006 & MR006-B-29947 & $7 / 28 / 11$ & Branch & ND & ND \\
\hline MR007 & MR007-B-18971 & $10 / 14 / 06$ & Branch & ND & 1.6 \\
\hline MR007 & MR007-L-18970 & $10 / 14 / 06$ & Leaf & ND & 2.0 \\
\hline MR007 & MR007-B-23162 & $4 / 2 / 07$ & Branch & ND & ND \\
\hline MR007 & MR007-B-23183 & $7 / 26 / 07$ & Branch & 0.1 & ND \\
\hline MR007 & MR007-L-23182 & $7 / 26 / 07$ & Leaf & ND & 1.3 \\
\hline MR007 & MR007-B-16626 & $7 / 24 / 08$ & Branch & ND & ND \\
\hline MR007 & MR007-B-29932 & $8 / 27 / 09$ & Branch & 0.7 & 0.8 \\
\hline MR007 & MR007-B-31932 & $7 / 27 / 10$ & Branch & ND & ND \\
\hline MR007 & MR007-B-29948 & $7 / 28 / 11$ & Branch & ND & ND \\
\hline MR008 & MR008-B-18973 & $10 / 14 / 06$ & Branch & ND & 1.3 \\
\hline MR008 & MR008-L-18972 & $10 / 14 / 06$ & Leaf & ND & 2.5 \\
\hline MR008 & MR008-B-23163 & $4 / 2 / 07$ & Branch & ND & 0.8 \\
\hline MR008 & MR008-B-23185 & $7 / 26 / 07$ & Branch & ND & ND \\
\hline MR008 & MR008-L-23184 & $7 / 26 / 07$ & Leaf & ND & ND \\
\hline MR008 & MR008-B-16627 & $7 / 24 / 08$ & Branch & ND & ND \\
\hline MR008 & MR008-B-29933 & $8 / 27 / 09$ & Branch & ND & ND \\
\hline MR008 & MR008-B-31933 & $7 / 27 / 10$ & Branch & ND & ND \\
\hline MR008 & MR008-B-29950 & $7 / 28 / 11$ & Branch & ND & ND \\
\hline MR009 & MR009-B-18975 & $10 / 14 / 06$ & Branch & ND & 0.9 \\
\hline MR009 & MR009-L-18974 & $10 / 14 / 06$ & Leaf & ND & 2.5 \\
\hline MR009 & MR009-B-23165 & $4 / 2 / 07$ & Branch & ND & ND \\
\hline MR009 & MR009-B-23189 & $7 / 26 / 07$ & Branch & ND & ND \\
\hline MR009 & MR009-L-23188 & $7 / 26 / 07$ & Leaf & ND & 1.5 \\
\hline MR009 & MR009-B-16629 & $7 / 24 / 08$ & Branch & ND & ND \\
\hline MR009 & MR009-B-29935 & $8 / 27 / 09$ & Branch & ND & ND \\
\hline MR009 & MR009-B-31935 & $7 / 27 / 10$ & Branch & ND & ND \\
\hline MR009 & MR009-B-29952 & $7 / 28 / 11$ & Branch & ND & ND \\
\hline MR010 & MR010-B-18977 & $10 / 14 / 06$ & Branch & ND & 3.4 \\
\hline MR010 & MR010-L-18976 & $10 / 14 / 06$ & Leaf & ND & ND \\
\hline MR010 & MR010-B-23167 & $4 / 2 / 07$ & Branch & ND & ND \\
\hline MR010 & MR010-B-23193 & $7 / 26 / 07$ & Branch & ND & 0.8 \\
\hline MR010 & MR010-L-23192 & $7 / 26 / 07$ & Leaf & ND & 1.4 \\
\hline MR010 & MR010-B-16631 & $7 / 24 / 08$ & Branch & 0.14 & 1.4 \\
\hline MR010 & MR010-B-29936 & $8 / 27 / 09$ & Branch & ND & ND \\
\hline MR010 & MR010-B-31936 & $7 / 27 / 10$ & Branch & ND & ND \\
\hline MR010 & MR010-B-29953 & $7 / 28 / 11$ & Branch & ND & ND \\
\hline MR011 & MR011-B-18979 & $10 / 14 / 06$ & Branch & ND & 2.2 \\
\hline MR011 & MR011-L-18978 & $10 / 14 / 06$ & Leaf & ND & 2.6 \\
\hline MR011 & MR011-B-23168 & $4 / 2 / 07$ & Branch & ND & ND \\
\hline
\end{tabular}


TABLE 3.5 (Cont.)

\begin{tabular}{|c|c|c|c|c|c|}
\hline \multirow[b]{2}{*}{ Location } & \multirow[b]{2}{*}{ Sample } & \multirow[b]{2}{*}{$\begin{array}{c}\text { Sample } \\
\text { Date }\end{array}$} & \multirow[b]{2}{*}{ Type } & \multicolumn{2}{|c|}{ Concentration $(\mu \mathrm{g} / \mathrm{kg})$} \\
\hline & & & & $\begin{array}{c}\text { Carbon } \\
\text { Tetrachloride }\end{array}$ & Chloroform \\
\hline MR011 & MR011-B-23195 & $7 / 26 / 07$ & Branch & ND & ND \\
\hline MR011 & MR011-L-23194 & $7 / 26 / 07$ & Leaf & ND & 1.7 \\
\hline MR011 & MR011-B-16632 & $7 / 24 / 08$ & Branch & ND & ND \\
\hline MR011 & MR011-B-29937 & $8 / 27 / 09$ & Branch & ND & ND \\
\hline MR011 & MR011-B-31937 & $7 / 27 / 10$ & Branch & ND & ND \\
\hline MR011 & MR011-B-29954 & $7 / 28 / 11$ & Branch & ND & ND \\
\hline MR012 & MR012-B-18981 & $10 / 14 / 06$ & Branch & ND & 2.1 \\
\hline MR012 & MR012-L-18980 & $10 / 14 / 06$ & Leaf & ND & 2.9 \\
\hline MR012 & MR012-B-23169 & $4 / 2 / 07$ & Branch & ND & ND \\
\hline MR012 & MR012-B-23197 & $7 / 26 / 07$ & Branch & ND & ND \\
\hline MR012 & MR012-L-23196 & $7 / 26 / 07$ & Leaf & ND & 1.4 \\
\hline MR012 & MR012-B-16633 & $7 / 24 / 08$ & Branch & ND & ND \\
\hline MR012 & MR012-B-29938 & $8 / 27 / 09$ & Branch & ND & ND \\
\hline MR012 & MR012-B-31938 & $7 / 27 / 10$ & Branch & ND & ND \\
\hline MR012 & MR012-B-29955 & $7 / 28 / 11$ & Branch & ND & ND \\
\hline MR013 & MR013-B-18983 & $10 / 14 / 06$ & Branch & ND & ND \\
\hline MR013 & MR013-L-18982 & $10 / 14 / 06$ & Leaf & ND & 2.2 \\
\hline MR013 & MR013-B-23160 & $4 / 2 / 07$ & Branch & ND & ND \\
\hline MR013 & MR013-B-23179 & $7 / 26 / 07$ & Branch & ND & ND \\
\hline MR013 & MR013-L-23178 & $7 / 26 / 07$ & Leaf & ND & ND \\
\hline MR013 & MR013-B-16624 & $7 / 24 / 08$ & Branch & ND & ND \\
\hline MR013 & MR013-B-29931 & $8 / 27 / 09$ & Branch & ND & ND \\
\hline MR013 & MR013-B-31930 & $7 / 27 / 10$ & Branch & ND & ND \\
\hline MR013 & MR013-B-29946 & $7 / 28 / 11$ & Branch & ND & ND \\
\hline MR014 & MR014-B-23164 & $4 / 2 / 07$ & Branch & ND & ND \\
\hline MR014 & MR014-B-23187 & $7 / 26 / 07$ & Branch & 0.3 & ND \\
\hline MR014 & MR014-L-23186 & $7 / 26 / 07$ & Leaf & ND & ND \\
\hline MR014 & MR014-B-16628 & $7 / 24 / 08$ & Branch & ND & ND \\
\hline MR014 & MR014-B-29934 & $8 / 27 / 09$ & Branch & ND & ND \\
\hline MR014 & MR014-B-31934 & $7 / 27 / 10$ & Branch & ND & ND \\
\hline MR014 & MR014-B-29951 & $7 / 28 / 11$ & Branch & ND & ND \\
\hline MR015 & MR015-B-23166 & $4 / 2 / 07$ & Branch & ND & 0.8 \\
\hline MR015 & MR015-B-23191 & $7 / 26 / 07$ & Branch & ND & ND \\
\hline MR015 & MR015-L-23190 & $7 / 26 / 07$ & Leaf & ND & ND \\
\hline MR015 & MR015-B-16630 & $7 / 24 / 08$ & Branch & ND & ND \\
\hline MR016 & MR016-B-23170 & $4 / 2 / 07$ & Branch & ND & 1.1 \\
\hline MR016 & MR016-B-23199 & $7 / 26 / 07$ & Branch & ND & ND \\
\hline MR016 & MR016-L-23198 & $7 / 26 / 07$ & Leaf & ND & ND \\
\hline MR016 & MR016-B-16634 & $7 / 24 / 08$ & Branch & ND & ND \\
\hline MR016 & MR016-B-29939 & $8 / 27 / 09$ & Branch & ND & ND \\
\hline MR016 & MR016-B-31939 & $7 / 27 / 10$ & Branch & ND & ND \\
\hline MR016 & MR016-B-29956 & $7 / 28 / 11$ & Branch & ND & ND \\
\hline MR017 & MR017-B-23171 & $4 / 2 / 07$ & Branch & ND & ND \\
\hline MR017 & MR017-B-23203 & $7 / 26 / 07$ & Branch & ND & ND \\
\hline MR017 & MR017-L-23202 & $7 / 26 / 07$ & Leaf & ND & ND \\
\hline MR017 & MR017-B-16635 & $7 / 24 / 08$ & Branch & ND & ND \\
\hline MR017 & MR017-B-29940 & $8 / 27 / 09$ & Branch & 0.1 & ND \\
\hline
\end{tabular}


TABLE 3.5 (Cont.)

\begin{tabular}{|c|c|c|c|c|c|}
\hline \multirow[b]{2}{*}{ Location } & \multirow[b]{2}{*}{ Sample } & \multirow[b]{2}{*}{$\begin{array}{c}\text { Sample } \\
\text { Date }\end{array}$} & \multirow[b]{2}{*}{ Type } & \multicolumn{2}{|c|}{ Concentration $(\mu \mathrm{g} / \mathrm{kg})$} \\
\hline & & & & $\begin{array}{c}\text { Carbon } \\
\text { Tetrachloride }\end{array}$ & Chloroform \\
\hline MR017 & MR017-B-31940 & $7 / 27 / 10$ & Branch & ND & ND \\
\hline MR017 & MR017-B-29957 & $7 / 28 / 11$ & Branch & ND & ND \\
\hline MR018 & MR018-B-23172 & $4 / 2 / 07$ & Branch & ND & ND \\
\hline MR018 & MR018-B-23201 & $7 / 26 / 07$ & Branch & ND & ND \\
\hline MR018 & MR018-L-23200 & $7 / 26 / 07$ & Leaf & ND & 3.2 \\
\hline MR018 & MR018-B-16636 & $7 / 24 / 08$ & Branch & ND & ND \\
\hline MR018 & MR018-B-29941 & 8/27/09 & Branch & 0.2 & ND \\
\hline MR018 & MR018-B-31941 & $7 / 27 / 10$ & Branch & ND & ND \\
\hline MR018 & MR018-B-29958 & $7 / 28 / 11$ & Branch & ND & 0.8 \\
\hline MR019 & MR019-B-16637 & $7 / 24 / 08$ & Branch & 0.1 & ND \\
\hline MR019 & MR019-B-29929 & 8/27/09 & Branch & ND & ND \\
\hline MR019 & MR019-B-31924 & $7 / 27 / 10$ & Branch & ND & ND \\
\hline MR019 & MR019-B-29945 & $7 / 28 / 11$ & Branch & ND & ND \\
\hline MR020 & MR020-B-20021 & $7 / 24 / 08$ & Branch & ND & ND \\
\hline MR020 & MR020-B-29928 & $8 / 27 / 09$ & Branch & ND & ND \\
\hline MR020 & MR020-B-31923 & $7 / 27 / 10$ & Branch & ND & ND \\
\hline MR020 & MR020-B-29982 & $7 / 28 / 11$ & Branch & ND & ND \\
\hline MR021 & MR021-B-20022 & $7 / 24 / 08$ & Branch & ND & ND \\
\hline MR021 & MR021-B-29926 & $8 / 27 / 09$ & Branch & ND & ND \\
\hline MR021 & MR021-B-31920 & $7 / 27 / 10$ & Branch & ND & ND \\
\hline MR021 & MR021-B-29967 & $7 / 28 / 11$ & Branch & ND & ND \\
\hline MR022 & MR022-B-20023 & $7 / 24 / 08$ & Branch & ND & ND \\
\hline MR023 & MR023-B-20024 & $7 / 24 / 08$ & Branch & ND & ND \\
\hline MR023 & MR023-B-29925 & $8 / 27 / 09$ & Branch & 0.2 & ND \\
\hline MR023 & MR023-B-31921 & $7 / 27 / 10$ & Branch & ND & ND \\
\hline MR024 & MR024-B-20025 & $7 / 24 / 08$ & Branch & ND & ND \\
\hline MR024 & MR024-B-29927 & 8/27/09 & Branch & ND & ND \\
\hline MR024 & MR024-B-31922 & $7 / 27 / 10$ & Branch & ND & ND \\
\hline MR024 & MR024-B-29981 & $7 / 28 / 11$ & Branch & ND & ND \\
\hline MR025 & MR025-B-31942 & $7 / 28 / 10$ & Branch & ND & ND \\
\hline MR025 & MR025-B-29968 & $7 / 28 / 11$ & Branch & ND & ND \\
\hline MR026 & MR026-B-31943 & $7 / 28 / 10$ & Branch & ND & ND \\
\hline MR026 & MR026-B-29969 & $7 / 28 / 11$ & Branch & ND & ND \\
\hline MR027 & MR027-B-31944 & $7 / 28 / 10$ & Branch & ND & ND \\
\hline MR027 & MR027-B-29970 & $7 / 28 / 11$ & Branch & ND & ND \\
\hline MR028 & MR028-B-31945 & $7 / 28 / 10$ & Branch & ND & ND \\
\hline MR028 & MR028-B-29971 & $7 / 28 / 11$ & Branch & ND & ND \\
\hline MR029 & MR029-B-31946 & $7 / 28 / 10$ & Branch & ND & ND \\
\hline MR029 & MR029-B-29972 & $7 / 28 / 11$ & Branch & ND & ND \\
\hline
\end{tabular}


TABLE 3.5 (Cont.)

\begin{tabular}{|c|c|c|c|c|c|}
\hline \multirow[b]{2}{*}{ Location } & \multirow[b]{2}{*}{ Sample } & \multirow[b]{2}{*}{$\begin{array}{c}\text { Sample } \\
\text { Date }\end{array}$} & \multirow[b]{2}{*}{ Type } & \multicolumn{2}{|c|}{ Concentration $(\mu \mathrm{g} / \mathrm{kg})$} \\
\hline & & & & $\begin{array}{c}\text { Carbon } \\
\text { Tetrachloride }\end{array}$ & Chloroform \\
\hline MR030 & MR030-B-31947 & $7 / 28 / 10$ & Branch & ND & ND \\
\hline MR030 & MR030-B-29973 & $7 / 28 / 11$ & Branch & ND & ND \\
\hline MR031 & MR031-B-31948 & $7 / 28 / 10$ & Branch & 0.7 & ND \\
\hline MR031 & MR031-B-29974 & $7 / 28 / 11$ & Branch & ND & ND \\
\hline MR032 & MR032-B-31949 & $7 / 28 / 10$ & Branch & ND & ND \\
\hline MR032 & MR032-B-29975 & $7 / 28 / 11$ & Branch & ND & ND \\
\hline MR033 & MR033-B-31950 & $7 / 28 / 10$ & Branch & ND & ND \\
\hline MR033 & MR033-B-29976 & $7 / 28 / 11$ & Branch & ND & ND \\
\hline MR034 & MR034-B-31951 & $7 / 28 / 10$ & Branch & ND & ND \\
\hline MR034 & MR034-B-29977 & $7 / 28 / 11$ & Branch & ND & ND \\
\hline MR035 & MR035-B-31952 & $7 / 28 / 10$ & Branch & ND & ND \\
\hline MR035 & MR035-B-29979 & $7 / 28 / 11$ & Branch & ND & ND \\
\hline MR036 & MR036-B-31953 & $7 / 28 / 10$ & Branch & ND & ND \\
\hline MR036 & MR036-B-29980 & $7 / 28 / 11$ & Branch & ND & ND \\
\hline MR037 & MR037-B-31954 & $7 / 28 / 10$ & Branch & ND & ND \\
\hline MR037 & MR037-B-29978 & $7 / 28 / 11$ & Branch & 0.2 & ND \\
\hline MR038 & MR038-B-31955 & $7 / 28 / 10$ & Branch & ND & ND \\
\hline MR038 & MR038-B-29964 & $7 / 28 / 11$ & Branch & ND & ND \\
\hline MR039 & MR039-B-31956 & $7 / 28 / 10$ & Branch & ND & ND \\
\hline MR039 & MR039-B-29965 & $7 / 28 / 11$ & Branch & ND & ND \\
\hline MR040 & MR040-B-31957 & $7 / 28 / 10$ & Branch & ND & ND \\
\hline MR040 & MR040-B-29966 & $7 / 28 / 11$ & Branch & ND & ND \\
\hline MR041 & MR041-B-31958 & $7 / 28 / 10$ & Branch & 2.2 & ND \\
\hline MR041 & MR041-B-29985 & $7 / 28 / 11$ & Branch & ND & ND \\
\hline MR042 & MR042-B-31959 & $7 / 28 / 10$ & Branch & ND & ND \\
\hline MR042 & MR042-B-29986 & $7 / 28 / 11$ & Branch & ND & ND \\
\hline MR043 & MR043-B-31960 & $7 / 28 / 10$ & Branch & ND & ND \\
\hline MR043 & MR043-B-29983 & $7 / 28 / 11$ & Branch & 0.2 & 1.0 \\
\hline MR044 & MR044-B-31961 & $7 / 28 / 10$ & Branch & 0.5 & ND \\
\hline MR044 & MR044-B-29984 & $7 / 28 / 11$ & Branch & ND & ND \\
\hline
\end{tabular}

a Analyses conducted at the AGEM Laboratory by modified EPA Method 5021 (headspace analysis on a gas chromatograph with electron capture detection).

b ND, not detected at the method reporting limit of $0.1 \mu \mathrm{g} / \mathrm{kg}$ for carbon tetrachloride or $0.75 \mu \mathrm{g} / \mathrm{kg}$ for chloroform. 


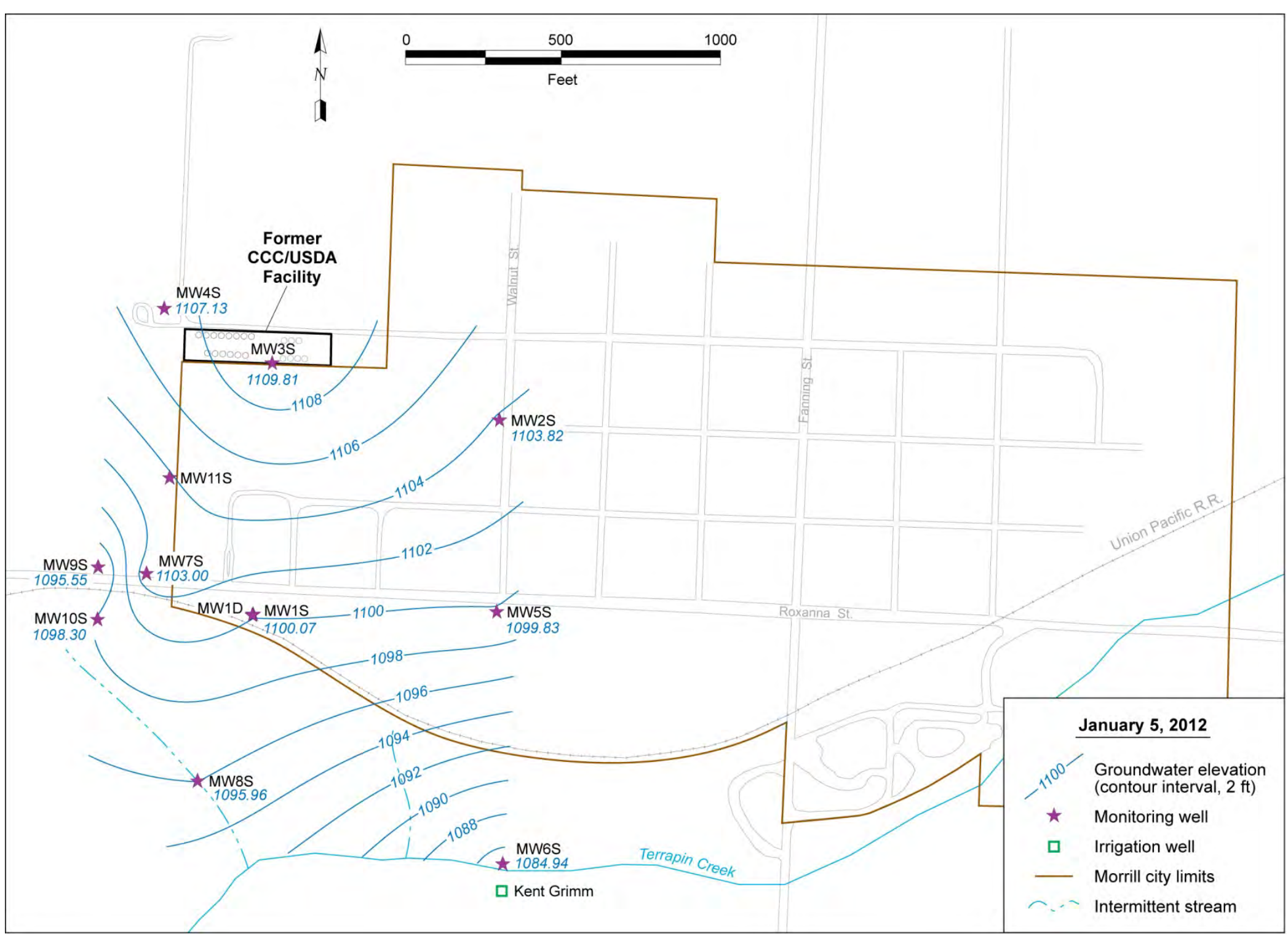

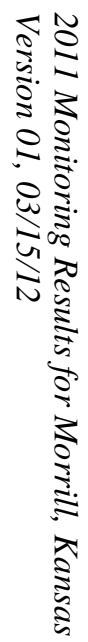

FIGURE 3.1 Potentiometric surface based on water levels measured manually on January 5, 2012. 


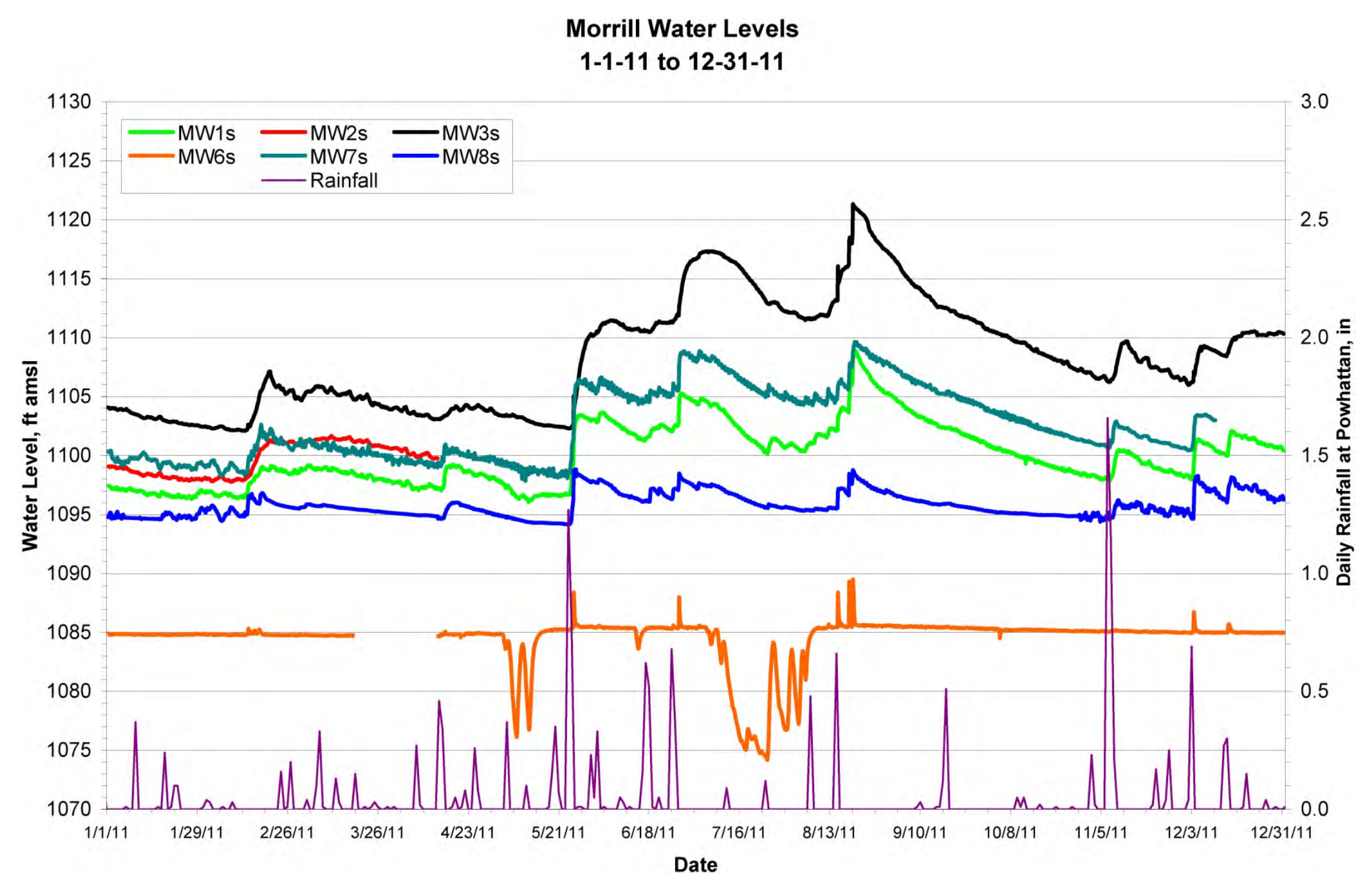

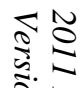

FIGURE 3.2 Hydrographs summarizing results of long-term water level monitoring from January 1, 2011, to December 31, 2011. 


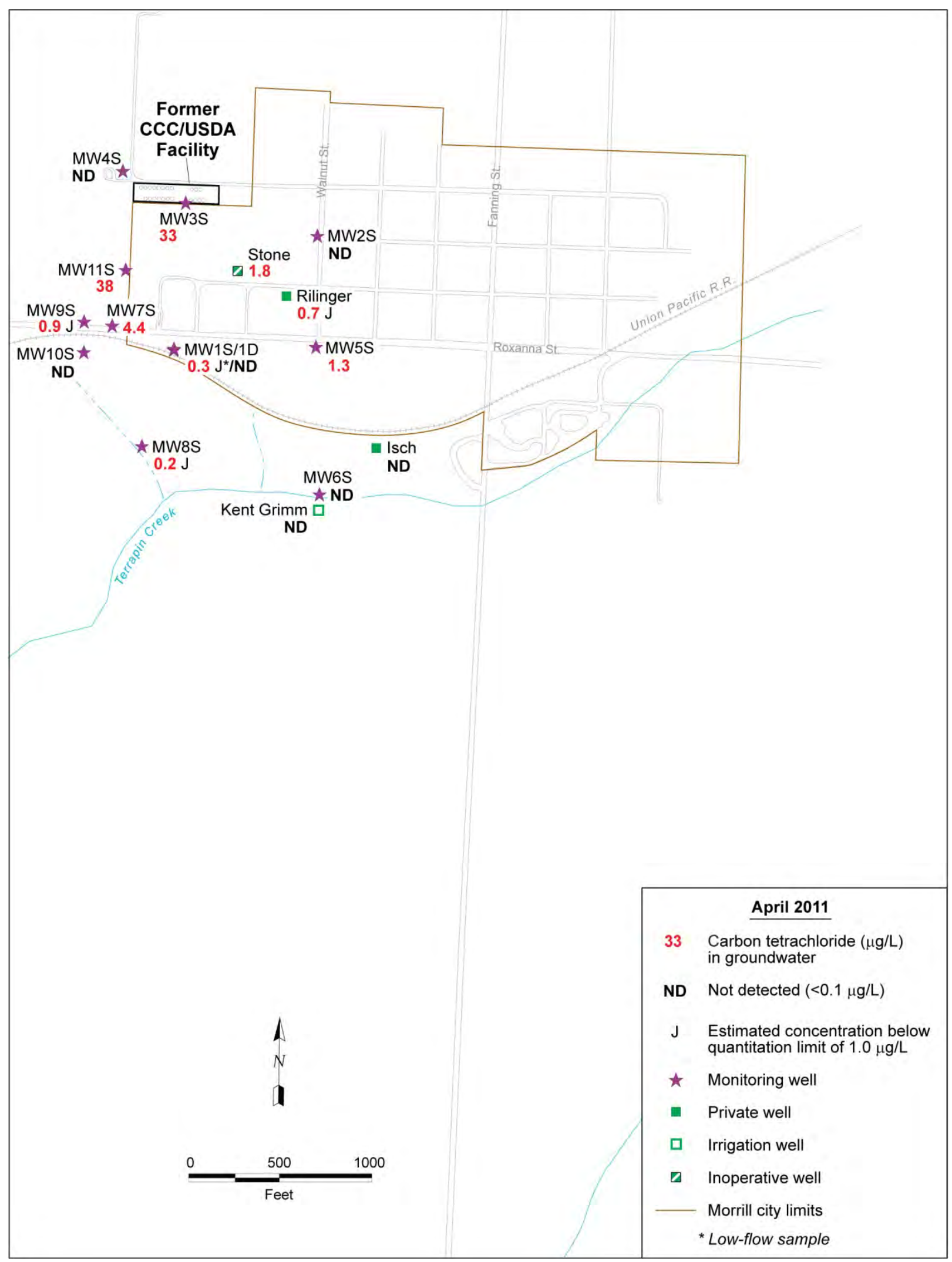

FIGURE 3.3a Carbon tetrachloride concentrations in groundwater, April 2011. 


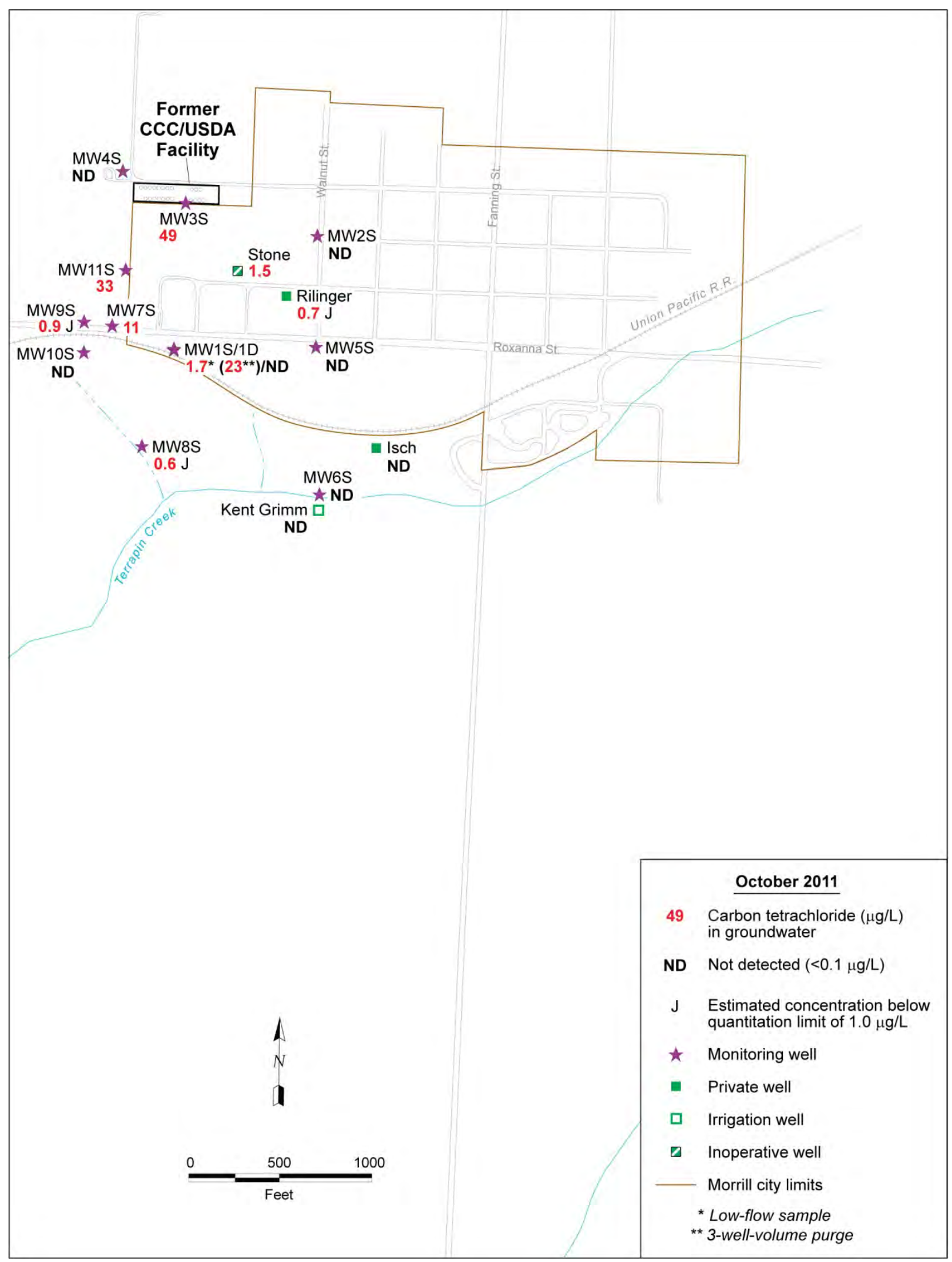

FIGURE 3.3b Carbon tetrachloride concentrations in groundwater, October 2011. 


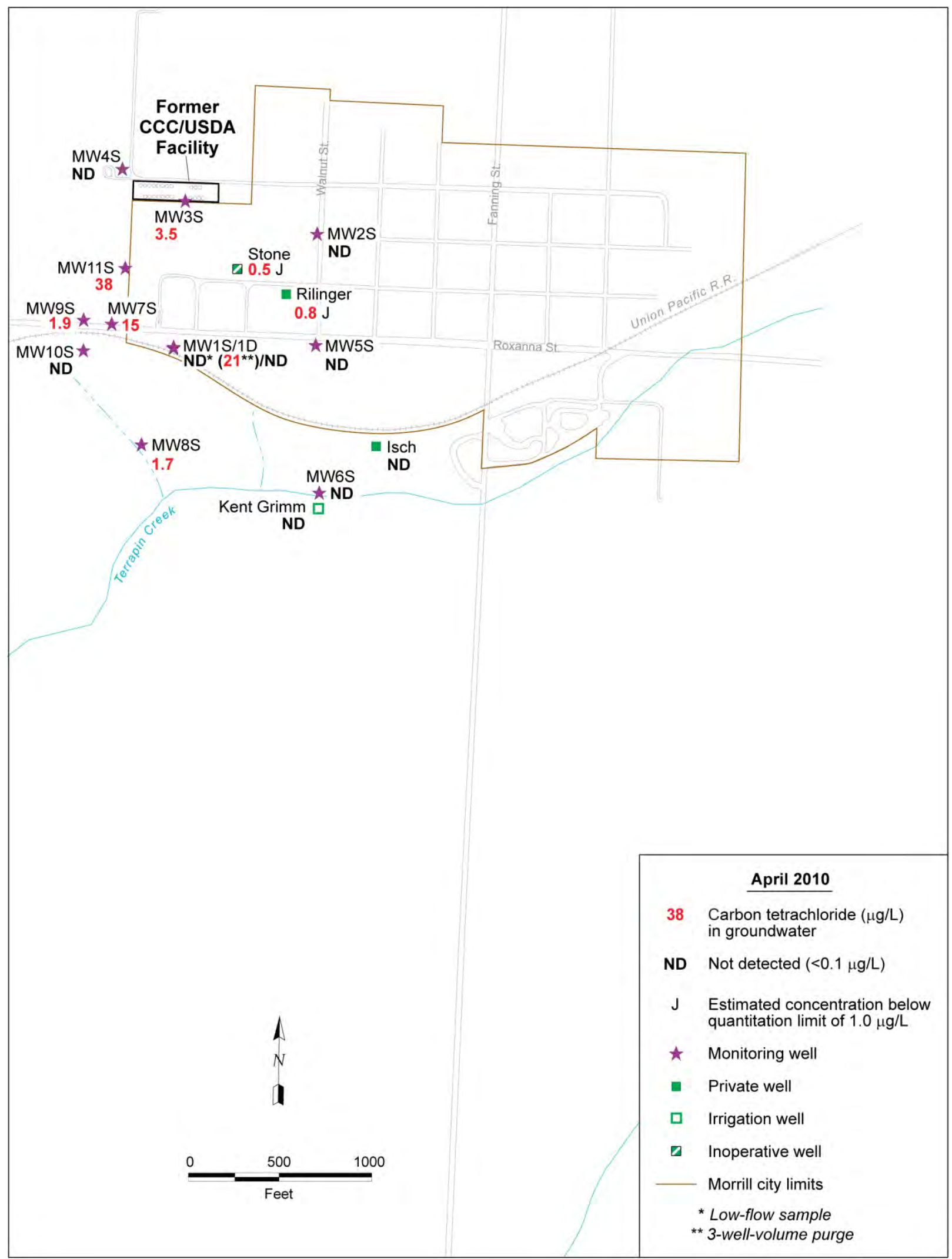

FIGURE 3.4a Carbon tetrachloride concentrations in groundwater, April 2010. 


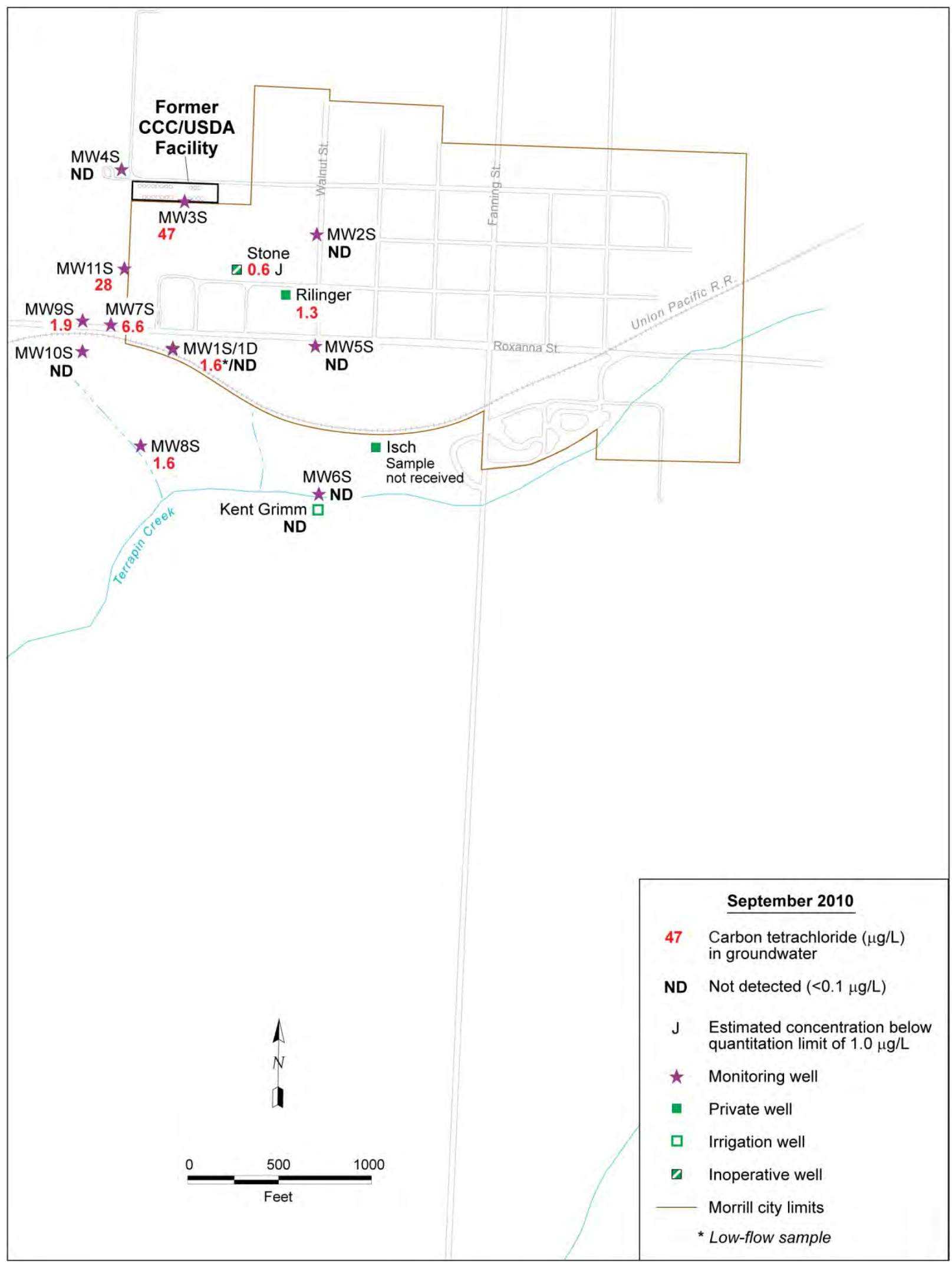

FIGURE 3.4b Carbon tetrachloride concentrations in groundwater, September 2010. 


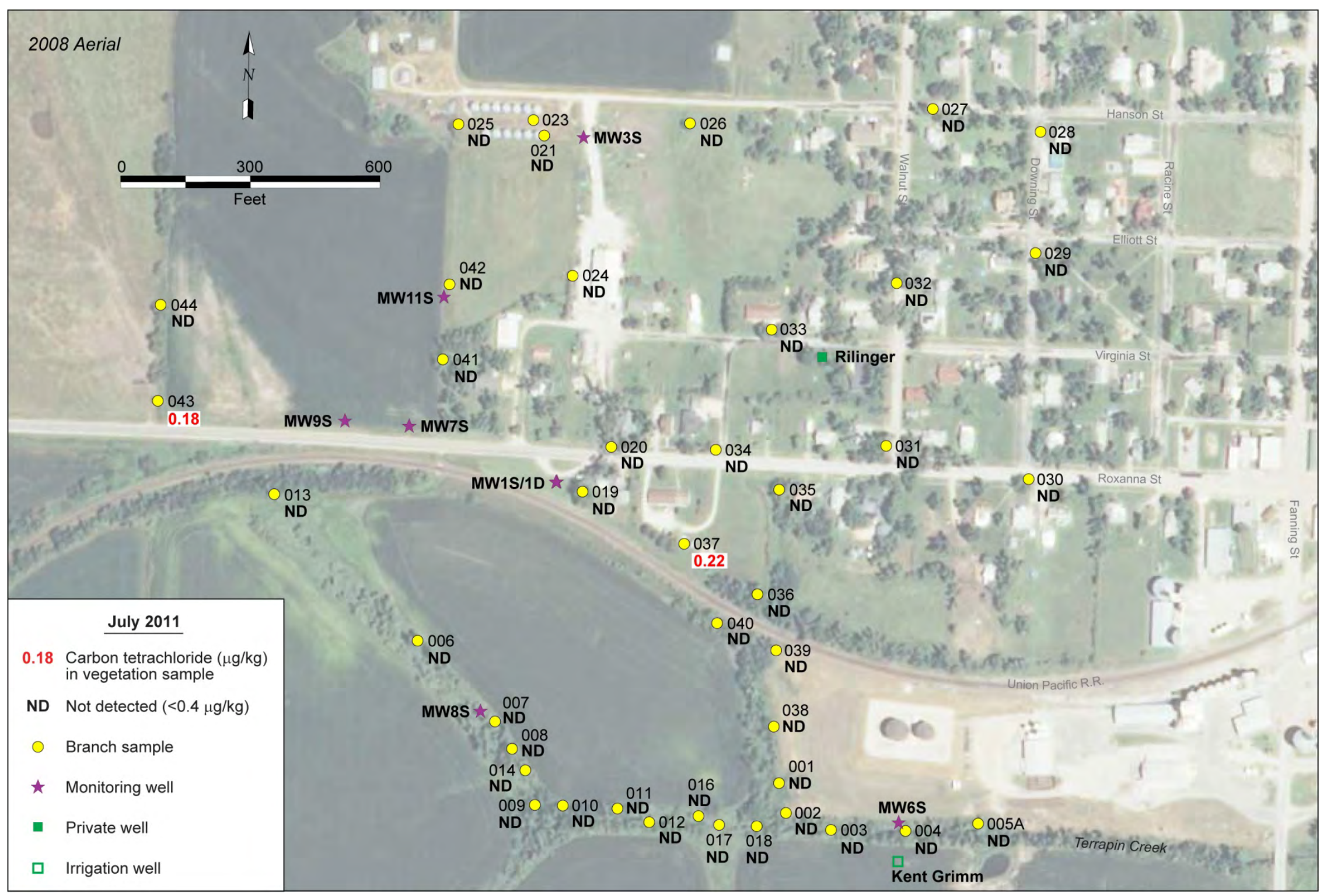

FIGURE 3.5 Carbon tetrachloride concentrations in vegetation, July 2011. 


\section{Conclusions and Recommendations}

\subsection{Conclusions}

The findings of the April 2011 and October 2011 monitoring events at Morrill support the following conclusions:

- Groundwater flow during the 2011 review period (as in prior years) was predominantly to the south, from the vicinity of the former CCC/USDA facility toward Terrapin Creek. Automatic water level monitoring data suggest that spring precipitation and recharge represent the predominant factors affecting the local groundwater level patterns.

- No significant changes were observed in the concentration or distribution of carbon tetrachloride in groundwater during the spring and fall 2011 monitoring events versus the spring and fall 2010 monitoring events. In October 2011, a maximum carbon tetrachloride concentration of $49 \mu \mathrm{g} / \mathrm{L}$ was identified in groundwater at well MW3S on the former CCC/USDA facility, with concentrations decreasing downgradient toward Terrapin Creek.

- $\quad$ Since 2004, the accumulated results of 15 sampling events have demonstrated a significant decline in the maximum detected concentration of carbon tetrachloride in groundwater. In 1995, the contaminant was detected at the former CCC/USDA facility at $390 \mu \mathrm{g} / \mathrm{L}$, while the current maximum levels are $<50 \mu \mathrm{g} / \mathrm{L}$. The residual contaminant plume extending from the former CCC/USDA facility southward toward Terrapin Creek is well-defined and slowly declining in concentration naturally.

- No carbon tetrachloride contamination was detected in 2011 in surface waters or shallow streambed sediments sampled at five locations along Terrapin Creek, downgradient from the former CCC/USDA facility. These results indicate that Terrapin Creek remains unaffected by the carbon tetrachloride plume. 
- Since 2007, the accumulated results of 10 monitoring events for surface water and sediment in Terrapin Creek have demonstrated no impact to the sediment and surface waters of the creek by carbon tetrachloride and no imminent risk for further degradation of the creek.

- Terrapin Creek (tributary segment 308 to Walnut Creek) receives discharge from the Morrill wastewater treatment plant and several confined animal feeding operations regulated by the KDHE. The Walnut Creek watershed is designated by the KDHE as impaired by fecal coliform bacteria. Terrapin Creek is classified by the KDHE as not open to or accessible by the public for contact recreation and does not support the food procurement designated use (KDHE 2010b).

- In July 2011, trace concentrations of carbon tetrachloride were detected in vegetation samples collected from trees at 2 of the 42 sampled locations south (downgradient) of the former CCC/USDA facility.

- Sampling of indoor air in August 2010 to evaluate the potential for vapor intrusion into homes overlying and within $100 \mathrm{ft}$ laterally of the identified carbon tetrachloride plume resulted in no detections of carbon tetrachloride. Low concentrations of chloroform, indicative of indoor air sources, were detected. Low radon levels were also detected. The results indicate no evidence of upward migration of vapors from the low-level carbon tetrachloride contamination in groundwater to indoor air.

\subsection{Recommendations}

\subsubsection{Groundwater Monitoring Frequency}

Figure 4.1 compares the carbon tetrachloride concentrations in groundwater samples collected in the monitoring network in October of 2003, 2007, and 2011. The results for these samples collected at 4-yr intervals demonstrate a stable areal distribution of carbon tetrachloride in groundwater and declining concentrations. Table 4.1 summarizes carbon tetrachloride concentrations in wells MW7S, MW8S, MW9S, and MW11S in fall sampling events in 2005- 
2011. Concentrations in these wells were cited by the KDHE (2010a) as a concern supporting the KDHE's request for continued twice yearly monitoring. The annual results in the table indicate decreasing concentration trends at these wells.

On the basis of this analysis, the CCC/USDA recommends a change in groundwater monitoring frequency from twice yearly to once yearly, at the locations established under the approved monitoring plan (Argonne 2005b). Annual monitoring of the carbon tetrachloride plume will be adequate to track future changes and will be protective. The annual events will involve sampling of groundwater from the existing network of 12 monitoring wells and 3 private wells, as well as sampling of surface waters and streambed sediments at the 5 established locations along Terrapin Creek.

\subsubsection{Vegetation Sampling Frequency}

Vegetation sampling was originally initiated to track plume migration. Other ongoing monitoring efforts are currently fulfilling that function, and after five years of sampling there appears to be no threat to surface water. The groundwater flow direction has been well established. Additionally, there are monitoring wells adjacent to and upgradient of surface water to track any change in contaminant concentrations, and the sampling of surface water and sediment has demonstrated the absence of carbon tetrachloride contamination in surface water. Therefore, the CCC/USDA recommends the elimination of vegetation sampling.

\subsubsection{Sampling Methods}

Comparisons of the low-flow and three-well-volume purging methods indicate that lowflow sampling provides representative results at the Morrill monitoring wells except for MW1S, which is located in the heart of the plume and is screened over a $40-\mathrm{ft}$ interval. Investigation of modified or alternative sampling methods will continue, so that representative samples for analysis can be reliably obtained from this well.

For the present, the CCC/USDA recommends continued sampling of well MW1S by the three-well-volume purging method and elimination of low-flow sampling for this one well. In addition, the CCC/USDA recommends deployment of passive diffusion bag sampler(s) in well 
MW1S during the spring 2012 sampling event for comparison with the three-well-volume purging method.

TABLE 4.1 Analytical results for carbon tetrachloride in groundwater samples collected at wells MW7S, MW8S, MW9S, and MW11S in September or October of years 20052011.

\begin{tabular}{lcccc}
\hline & \multicolumn{4}{c}{ Carbon Tetrachloride $(\mu \mathrm{g} / \mathrm{L})$ at Indicated Well } \\
\cline { 2 - 5 } Fall of Year & MW7S & MW8S & MW9S & MW11S \\
& & & & \\
\hline & & & & \\
2005 & 1.1 & $0.9 \mathrm{~J}^{\mathrm{a}}$ & - & - \\
2006 & 38 & 1.3 & $\mathrm{ND}^{\mathrm{b}}$ & 53 \\
2007 & 8.1 & $0.8 \mathrm{~J}$ & $\mathrm{ND}$ & 54 \\
2009 & 7.9 & 1.3 & 1.1 & 42 \\
2010 & 8.0 & 1.9 & 1.4 & 39 \\
2011 & 6.6 & 1.6 & 1.9 & 28 \\
& 11 & $0.6 \mathrm{~J}$ & 1.1 & 33 \\
\hline
\end{tabular}

a Qualifier J indicates an estimated concentration below the method quantitation limit of $1.0 \mu \mathrm{g} / \mathrm{L}$.

b ND, not detected at an instrument detection limit of $0.1 \mu \mathrm{g} / \mathrm{L}$. 


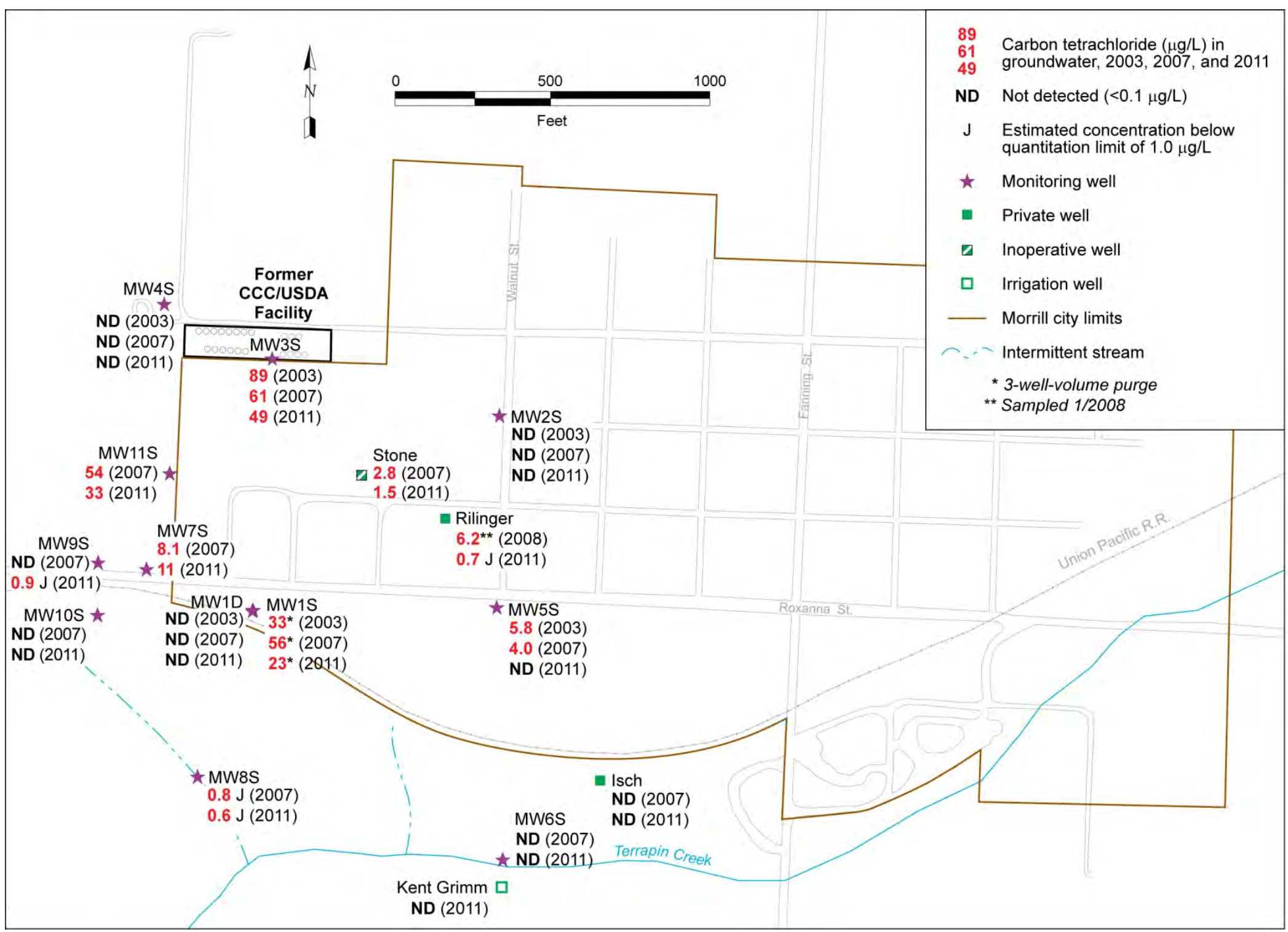

FIGURE 4.1 Carbon tetrachloride concentrations in groundwater in October of 2003, 2007, and 2011. 


\section{References}

Alvarado, J.S., and C. Rose, 2004, "Static Headspace Analysis of Volatile Organic Compounds in Soil and Vegetation Samples for Site Characterization,” Talanta 62:17-23.

Argonne, 2002, Final Master Work Plan: Environmental Investigations at Former CCC/USDA Facilities in Kansas, 2002 Revision, ANL/ER/TR-02/004, prepared for the Commodity Credit Corporation, U.S. Department of Agriculture, Washington, D.C., by Argonne National Laboratory, Argonne, Illinois, December.

Argonne, 2003, Final Work Plan: Phase I Expedited Site Characterization, Morrill, Kansas, ANL/ER/TR-03/001, prepared for the Commodity Credit Corporation, U.S. Department of Agriculture, Washington, D.C., by Argonne National Laboratory, Argonne, Illinois, September.

Argonne, 2004, Final Phase I-Phase II Interim Report: Expedited Site Characterization, Morrill, Kansas, ANL/ER/TR-04/001, prepared for the Commodity Credit Corporation, U.S. Department of Agriculture, Washington, D.C., by Argonne National Laboratory, Argonne, Illinois, December.

Argonne, 2005a, Final Report, Monitoring Well Installation and Sampling, 2004, at Morrill, Kansas, ANL/ER/TR-04/010, prepared for the Commodity Credit Corporation, U.S. Department of Agriculture, Washington, D.C., by Argonne National Laboratory, Argonne, Illinois, November.

Argonne, 2005b, Final Work Plan: Groundwater Monitoring at Morrill, Kansas, ANL/ER/TR05/003, prepared for the Commodity Credit Corporation, U.S. Department of Agriculture, Washington, D.C., by Argonne National Laboratory, Argonne, Illinois, August.

Argonne, 2006, September 2006 Monitoring Results for Morrill, Kansas, ANL/EVS/AGEM/CHRON-1016, prepared for the Commodity Credit Corporation, U.S. Department of Agriculture, Washington, D.C., by Argonne National Laboratory, Argonne, Illinois, December.

Argonne, 2007a, Final Report: Groundwater Monitoring at Morrill, Kansas, in September 2005 and March 2006, with Expansion of the Monitoring Network in January 2006, 
ANL/EVS/AGEM/TR-06-09, prepared for the Commodity Credit Corporation, U.S. Department of Agriculture, Washington, D.C., by Argonne National Laboratory, Argonne, Illinois, June.

Argonne, 2007b, March-April 2007 Monitoring Results for Morrill, Kansas, ANL/EVS/AGEM/TR-07-06, prepared for the Commodity Credit Corporation, U.S. Department of Agriculture, by Argonne National Laboratory, Argonne, Illinois, May.

Argonne, 2008a, October 2007 Monitoring Results for Morrill, Kansas, ANL/EVS/AGEM/TR08-02, prepared for the Commodity Credit Corporation, U.S. Department of Agriculture, Washington, D.C., by Argonne National Laboratory, Argonne, Illinois, March.

Argonne, 2008b, April 2008 Monitoring Results for Morrill, Kansas, ANL/EVS/AGEM/TR-0815, prepared for the Commodity Credit Corporation, U.S. Department of Agriculture, Washington, D.C., by Argonne National Laboratory, Argonne, Illinois, July.

Argonne, 2009, October 2008 Monitoring Results for Morrill, Kansas, ANL/EVS/AGEM/TR09-03, prepared for the Commodity Credit Corporation, U.S. Department of Agriculture, Washington, D.C., by Argonne National Laboratory, Argonne, Illinois, March.

Argonne, 2010, Annual Report of Monitoring at Morrill, Kansas, ANL/EVS/AGEM/TR-10-04, prepared for the Commodity Credit Corporation, U.S. Department of Agriculture, Washington, D.C., by Argonne National Laboratory, Argonne, Illinois, August.

Argonne, 2011, Annual Report of Monitoring at Morrill, Kansas, in 2010, ANL/EVS/AGEM/TR-11-06, prepared for the Commodity Credit Corporation, U.S. Department of Agriculture, Washington, D.C., by Argonne National Laboratory, Argonne, Illinois, June.

EPA, 1995, Method 524.2: Measurement of Purgeable Organic Compounds in Water by Capillary Column Gas Chromatography/Mass Spectrometry, Revision 4.1, edited by J.W. Munch, National Exposure Research Laboratory, Office of Research and Development, U.S. Environmental Protection Agency, Cincinnati, Ohio.

GeoCore, 1994a, Work Plan: Phase I Sampling and Site Visit: Brown County Groundwater Investigation, prepared for the Kansas Department of Health and Environment, Bureau of 
Environmental Remediation, Topeka, Kansas, by GeoCore Services, Inc., Salina, Kansas, April 12.

GeoCore, 1994b, Well Survey Material: Brown County Groundwater Investigation: Morrill PWS Well \#5 Site, field notes made by GeoCore Services, Inc., Salina, Kansas, during Phase I sampling of private wells, May 19.

GeoCore, 1994c, Work Plan: Phase II Drilling and Sampling: Brown County Groundwater Investigation, prepared for the Kansas Department of Health and Environment, Bureau of Environmental Remediation, Topeka, Kansas, by GeoCore Services, Inc., Salina, Kansas, July 14.

GeoCore, 1994d, Preliminary Report: Phase I Sampling: Brown County Groundwater Investigation, prepared for the Kansas Department of Health and Environment, Bureau of Environmental Remediation, Topeka, Kansas, by GeoCore Services, Inc., Salina, Kansas, July 19.

GeoCore, 1996, Environmental Site Investigation Report: Phase II Drilling and Sampling: Morrill Public Water Supply Well \#5, Morrill, Kansas, prepared for the Kansas Department of Health and Environment, Bureau of Environmental Remediation, Topeka, Kansas, by GeoCore Services, Inc., Salina, Kansas, February 29.

KDHE, 1989, Preliminary Assessment of the Morrill Public Water Supply Well \#5, Morrill, Kansas (Site Identification Number: KS D981710288), Kansas Department of Health and Environment, Bureau of Environmental Remediation, Topeka, Kansas, June 6.

KDHE 2001, Missouri Basin Total Maximum Daily Load: Waterbody: Walnut Creek: Water Quality Impairment: Fecal Coliform Bacteria, Bureau of Water, Kansas Department of Health and Environment, Topeka, Kansas, August 28 (http://www.kdheks.gov/tmdl/mo/ WalnutCrFCB.pdf).

KDHE, 2005, letter from C. Carey (Bureau of Environmental Remediation, Kansas Department of Health and Environment, Topeka, Kansas) to C. Roe (Commodity Credit Corporation, U.S. Department of Agriculture, Washington, D.C.) regarding Final Work Plan: Groundwater Monitoring at Morrill, Kansas, October 31. 
KDHE, 2007a, letter from C. Carey (Bureau of Environmental Remediation, Kansas Department of Health and Environment, Topeka, Kansas) to C. Roe (Commodity Credit Corporation, U.S. Department of Agriculture, Washington, D.C.) regarding draft Morrill sampling report, January 5.

KDHE, 2007b, letter from C. Carey (Bureau of Environmental Remediation, Kansas Department of Health and Environment, Topeka, Kansas) to C. Roe (Commodity Credit Corporation, U.S. Department of Agriculture, Washington, D.C.) regarding March-April 2007 Monitoring Results for Morrill, Kansas, August 1.

KDHE, 2008a, letter from E. McWilliams (Bureau of Environmental Remediation, Kansas Department of Health and Environment, Topeka, Kansas) to C. Roe (Commodity Credit Corporation, U.S. Department of Agriculture, Washington, D.C.) regarding April 2008 Monitoring Report for Morrill, Kansas, August 6.

KDHE, 2008b, letter from C. Carey (Bureau of Environmental Remediation, Kansas Department of Health and Environment, Topeka, Kansas) to C. Roe (Commodity Credit Corporation, U.S. Department of Agriculture, Washington, D.C.) regarding revisions to the Morrill sampling report for September 2005 and March 2006, February 6.

KDHE, 2010a, letter from M. Townsend (Bureau of Environmental Remediation, Kansas Department of Health and Environment, Topeka, Kansas) to C. Roe (Commodity Credit Corporation, U.S. Department of Agriculture, Washington, D.C.) regarding Annual Report of Monitoring at Morrill, Kansas, in 2009, September 28.

KDHE, 2010b, Kansas Surface Water Register, Bureau of Environmental Field Services, Kansas Department of Health and Environment, Topeka, Kansas, December 15 (http://www.kdheks.gov/ befs/download/Current_Kansas_Surface_Register.pdf).

KDHE, 2011, letter from M. Townsend (Bureau of Environmental Remediation, Kansas Department of Health and Environment, Topeka, Kansas) to C. Roe (Commodity Credit Corporation, U.S. Department of Agriculture, Washington, D.C.) regarding Annual Report of Monitoring at Morrill, Kansas, in 2010, July 28. 
Puls, R.W., and Barcelona, M.J., 1996, “Low-Flow (Minimal Drawdown) Ground-Water Sampling Procedures,” EPA/540/S-95/504, in Ground Water Issue, Superfund Technology Support Center for Ground Water, National Risk Management Research Laboratory, Ada, Oklahoma, April (www.epa.gov/tio/tsp/download/lwflw2a.pdf).

Yeskis, D., and B. Zavala, 2002, Ground-Water Sampling Guidelines for Superfund and RCRA Project Managers: Ground Water Forum Issue Paper, EPA 542-S-02-001, Technology Innovative Office, Office of Solid Waste and Emergency Response, U.S. Environmental Protection Agency, Washington, D.C., May (http://www.epa.gov/tio/tsp/download/ gw_sampling_guide.pdf). 
Appendix A:

Sampling Activities at Morrill in 2011 
TABLE A.1 Sequence of sampling activities in 2011.

\begin{tabular}{|c|c|c|c|c|c|c|c|c|c|}
\hline \multicolumn{2}{|c|}{$\begin{array}{c}\text { Sample } \\
\text { Date and Time }\end{array}$} & Sample & Medium $^{a}$ & Type $^{a}$ & Location & $\begin{array}{l}\text { Depth } \\
\text { (ft BGL) }\end{array}$ & $\begin{array}{l}\text { Chain of } \\
\text { Custody }\end{array}$ & $\begin{array}{l}\text { Shipping } \\
\text { Date }\end{array}$ & Sample Description \\
\hline $4 / 20 / 11$ & 9:00 & MRSM4-W-30036 & Water & SW & SM4 & - & 4824 & $4 / 20 / 11$ & Water sample from Terrapin Creek. \\
\hline $4 / 20 / 11$ & $9: 01$ & MRSM4-S-30036 & Sediment & Sed & SM4 & - & 4823 & $4 / 20 / 11$ & Sediment sample from Terrapin Creek. \\
\hline $4 / 20 / 11$ & $9: 08$ & MRSM3-W-30035 & Water & SW & SM3 & - & 4824 & $4 / 20 / 11$ & Water sample from Terrapin Creek. \\
\hline $4 / 20 / 11$ & $9: 09$ & MRSM3-S-30035 & Sediment & Sed & SM3 & - & 4823 & $4 / 20 / 11$ & Sediment sample from Terrapin Creek. \\
\hline $4 / 20 / 11$ & 9:15 & MRSM2-W-30034 & Water & SW & SM2 & - & 4824 & $4 / 20 / 11$ & Water sample from Terrapin Creek. \\
\hline $4 / 20 / 11$ & 9:16 & MRSM2-S-30034 & Sediment & Sed & SM2 & - & 4823 & $4 / 20 / 11$ & Sediment sample from Terrapin Creek. \\
\hline $4 / 20 / 11$ & 9:28 & MRSM1-W-30033 & Water & SW & SM1 & - & 4824 & $4 / 20 / 11$ & Water sample from Terrapin Creek. \\
\hline $4 / 20 / 11$ & 9:29 & MRSM1-S-30033 & Sediment & Sed & SM1 & - & 4823 & $4 / 20 / 11$ & Sediment sample from Terrapin Creek. \\
\hline $4 / 20 / 11$ & 9:36 & MRSMB-W-30037 & Water & SW & SMB & - & 4824 & $4 / 20 / 11$ & Water sample from Terrapin Creek. \\
\hline $4 / 20 / 11$ & $9: 37$ & MRSMB-S-30037 & Sediment & Sed & SMB & - & 4823 & $4 / 20 / 11$ & Sediment sample from Terrapin Creek. \\
\hline $4 / 20 / 11$ & $11: 27$ & MRMW8S-W-30046 & Water & MW & MW8S & $10-25$ & 4825 & $4 / 21 / 11$ & $\begin{array}{l}\text { Depth to water }=2.73 \mathrm{ft} \text {. Depth of } 4 \text {-in. well = } \\
26.82 \mathrm{ft} \text {. Sample collected by using low-flow } \\
\text { bladder pump after purging of } 9 \mathrm{~L} \text {. Pump } \\
\text { intake positioned at } 17.5 \mathrm{ft} \text {. }\end{array}$ \\
\hline $4 / 20 / 11$ & $11: 38$ & MRMW1D-W-30039 & Water & MW & MW1D & $63-88$ & 4824 & $4 / 20 / 11$ & $\begin{array}{l}\text { Depth to water }=30.15 \mathrm{ft} \text {. Depth of } 4 \text {-in. well = } \\
89 \mathrm{ft} \text {. Sample collected by using low-flow } \\
\text { bladder pump after purging of } 5.5 \mathrm{~L} \text {. Pump } \\
\text { intake positioned at } 75.5 \mathrm{ft} \text {. }\end{array}$ \\
\hline $4 / 20 / 11$ & $12: 34$ & MRMW10S-W-30048 & Water & MW & MW10S & $30-45$ & 4825 & $4 / 21 / 11$ & $\begin{array}{l}\text { Depth to water }=13.69 \mathrm{ft} \text {. Depth of } 2 \text {-in. well }= \\
49.32 \mathrm{ft} \text {. Sample collected by using low-flow } \\
\text { bladder pump after purging of } 7.25 \mathrm{~L} \text {. Pump } \\
\text { intake positioned at } 37.5 \mathrm{ft} \text {. }\end{array}$ \\
\hline $4 / 20 / 11$ & $12: 36$ & MRMW1S-W-30038 & Water & MW & MW1S & $11-51$ & 4824 & $4 / 20 / 11$ & $\begin{array}{l}\text { Depth to water }=23.45 \mathrm{ft} \text {. Depth of } 4 \text {-in. well }= \\
54 \mathrm{ft} \text {. Sample collected by using low-flow } \\
\text { bladder pump after purging of } 6.5 \mathrm{~L} \text {. Pump } \\
\text { intake positioned at } 37 \mathrm{ft} \text {. }\end{array}$ \\
\hline $4 / 20 / 11$ & $13: 22$ & MRQCTB-W-30060 b & Water & TB & QC & - & 4824 & $4 / 20 / 11$ & $\begin{array}{l}\text { Trip blank sent to the AGEM Laboratory for } \\
\text { organic analysis with water samples listed on } \\
\text { chain-of-custody form (COC) } 4324 \text {. }\end{array}$ \\
\hline $4 / 20 / 11$ & $13: 39$ & MRMW6S-W-30044 & Water & MW & MW6S & $10-25$ & 4825 & $4 / 21 / 11$ & $\begin{array}{l}\text { Depth to water }=6.52 \mathrm{ft} \text {. Depth of } 4 \text {-in. well = } \\
24.88 \mathrm{ft} \text {. Sample collected by using low-flow } \\
\text { bladder pump after purging of } 8 \mathrm{~L} \text {. Pump } \\
\text { intake positioned at } 17.5 \mathrm{ft} \text {. }\end{array}$ \\
\hline $4 / 20 / 11$ & $14: 32$ & MRMW3S-W-30041 & Water & MW & MW3S & $18-48$ & 4825 & $4 / 21 / 11$ & $\begin{array}{l}\text { Depth to water }=32.06 \mathrm{ft} \text {. Depth of } 4 \text {-in. well }= \\
47.8 \mathrm{ft} \text {. Sample collected by using low-flow } \\
\text { bladder pump after purging of } 6.2 \mathrm{~L} \text {. Pump } \\
\text { intake positioned at } 40 \mathrm{ft} \text {. }\end{array}$ \\
\hline
\end{tabular}




\begin{tabular}{|c|c|c|c|c|c|c|c|c|c|}
\hline \multicolumn{2}{|c|}{$\begin{array}{c}\text { Sample } \\
\text { Date and Time }\end{array}$} & Sample & Medium $^{\mathrm{a}}$ & Type $^{\mathrm{a}}$ & Location & $\begin{array}{l}\text { Depth } \\
\text { (ft BGL) }\end{array}$ & $\begin{array}{l}\text { Chain of } \\
\text { Custody }\end{array}$ & $\begin{array}{l}\text { Shipping } \\
\text { Date }\end{array}$ & Sample Description \\
\hline $4 / 20 / 11$ & $15: 06$ & MRMW11S-W-30049 & Water & MW & MW11S & $53-68$ & 4825 & $4 / 21 / 11$ & $\begin{array}{l}\text { Depth to water }=39.16 \mathrm{ft} \text {. Depth of } 2 \text {-in. well = } \\
72.54 \mathrm{ft} \text {. Sample collected by using low-flow } \\
\text { bladder pump after purging of } 7.5 \mathrm{~L} \text {. Pump } \\
\text { intake positioned at } 60.5 \mathrm{ft} \text {. }\end{array}$ \\
\hline $4 / 20 / 11$ & $15: 45$ & MRQCIR-W-30056 & Water & $\mathrm{RI}$ & QC & - & 4825 & $4 / 21 / 11$ & $\begin{array}{l}\text { Rinsate of decontaminated sampling line after } \\
\text { collection of sample MRMW11S-W-30049. }\end{array}$ \\
\hline $4 / 20 / 11$ & $16: 34$ & MRMW5S-W-30043 & Water & MW & MW5S & $15-55$ & 4825 & $4 / 21 / 11$ & $\begin{array}{l}\text { Depth to water }=24.43 \mathrm{ft} \text {. Depth of } 4 \text {-in. well = } \\
54.6 \mathrm{ft} \text {. Sample collected by using low-flow } \\
\text { bladder pump after purging of } 7 \mathrm{~L} \text {. Pump } \\
\text { intake positioned at } 36 \mathrm{ft} \text {. }\end{array}$ \\
\hline $4 / 20 / 11$ & $16: 42$ & MRQCIR-W-30058b & Water & RI & QC & - & 4825 & $4 / 21 / 11$ & $\begin{array}{l}\text { Rinsate of decontaminated sampling line after } \\
\text { collection of sample MRMW5S-W-30043. }\end{array}$ \\
\hline $4 / 20 / 11$ & $17: 25$ & MRMW9S-W-30047 & Water & MW & MW9S & $\begin{array}{l}38.83- \\
53.83\end{array}$ & 4825 & $4 / 21 / 11$ & $\begin{array}{l}\text { Depth to water }=23.76 \mathrm{ft} \text {. Depth of } 2 \text {-in. well = } \\
58.32 \mathrm{ft} \text {. Sample collected by using low-flow } \\
\text { bladder pump after purging of } 14 \mathrm{~L} \text {. Pump } \\
\text { intake positioned at } 46.33 \mathrm{ft} \text {. }\end{array}$ \\
\hline $4 / 20 / 11$ & $17: 26$ & MRMW9SDUP-W-30054b & Water & MW & MW9S & $\begin{array}{l}38.83- \\
53.83\end{array}$ & 4825 & $4 / 21 / 11$ & Replicate of sample MRMW9S-W-30047. \\
\hline $4 / 20 / 11$ & $17: 36$ & MRMW2S-W-30040 & Water & MW & MW2S & $13-53$ & 4825 & $4 / 21 / 11$ & $\begin{array}{l}\text { Depth to water }=36.00 \mathrm{ft} \text {. Depth of } 4 \text {-in. well = } \\
53.5 \mathrm{ft} \text {. Sample collected by using low-flow } \\
\text { bladder pump after purging of } 8 \mathrm{~L} \text {. Pump } \\
\text { intake positioned at } 44.5 \mathrm{ft} \text {. }\end{array}$ \\
\hline $4 / 20 / 11$ & $17: 37$ & MRMW2SDUP-W-30055b & Water & MW & MW2S & $13-53$ & 4825 & $4 / 21 / 11$ & Replicate of sample MRMW2S-W-30040. \\
\hline $4 / 20 / 11$ & $18: 42$ & MRMW7S-W-30045 & Water & MW & MW7S & $20-45$ & 4825 & $4 / 21 / 11$ & $\begin{array}{l}\text { Depth to water }=19.69 \mathrm{ft} \text {. Depth of } 4 \text {-in. well = } \\
46.69 \mathrm{ft} \text {. Sample collected by using low-flow } \\
\text { bladder pump after purging of } 6 \mathrm{~L} \text {. Pump } \\
\text { intake positioned at } 32.5 \mathrm{ft} .\end{array}$ \\
\hline $4 / 20 / 11$ & 19:15 & MRTD12-W-30053 & Water & DW & TD12 & $27-67$ & 4825 & $4 / 21 / 11$ & $\begin{array}{l}\text { Water collected from Grimm well overflow before } \\
\text { the catch pond. }\end{array}$ \\
\hline $4 / 21 / 11$ & $9: 40$ & MRSTONE-W-30052 & Water & DW & Stone & 43 & 4826 & $4 / 21 / 11$ & Purged 5 gal with bailer and then sampled. \\
\hline $4 / 21 / 11$ & 9:56 & MRRILLINGER-W-30051 & Water & DW & Rillinger & - & 4826 & $4 / 21 / 11$ & $\begin{array}{l}\text { Allowed pump to run for } 5 \text { min and then collected } \\
\text { a grab sample. }\end{array}$ \\
\hline $4 / 21 / 11$ & $10: 32$ & MRISCH-W-30050 & Water & DW & Isch & - & 4826 & $4 / 21 / 11$ & $\begin{array}{l}\text { Allowed pump to run for } 5 \text { min. Co-op has been } \\
\text { using well for last several days. }\end{array}$ \\
\hline $4 / 21 / 11$ & $10: 42$ & MRMW4S-W-30042 & Water & MW & MW4S & $17-47$ & 4826 & $4 / 21 / 11$ & $\begin{array}{l}\text { Depth to water }=42.39 \mathrm{ft} \text {. Depth of } 4 \text {-in. well = } \\
47.8 \mathrm{ft} \text {. Sample collected by using low-flow } \\
\text { bladder pump after purging of } 5 \mathrm{~L} \text {. Pump } \\
\text { intake positioned at } 44.72 \mathrm{ft} \text {. }\end{array}$ \\
\hline
\end{tabular}




\begin{tabular}{|c|c|c|c|c|c|c|c|c|c|}
\hline \multicolumn{2}{|c|}{$\begin{array}{c}\text { Sample } \\
\text { Date and Time }\end{array}$} & Sample & \multirow{2}{*}{$\begin{array}{l}\text { Medium }^{\mathrm{a}} \\
\text { Water }\end{array}$} & \multirow{2}{*}{$\begin{array}{c}\text { Type }^{\mathrm{a}} \\
\text { FB }\end{array}$} & \multirow{2}{*}{$\begin{array}{l}\text { Location } \\
\text { QC }\end{array}$} & \multirow{2}{*}{$\begin{array}{c}\text { Depth } \\
\text { (ft BGL) } \\
\\
-\end{array}$} & \multirow{2}{*}{$\begin{array}{c}\text { Chain of } \\
\text { Custody }\end{array}$} & \multirow{2}{*}{$\begin{array}{c}\begin{array}{c}\text { Shipping } \\
\text { Date }\end{array} \\
4 / 21 / 11\end{array}$} & \multirow[t]{2}{*}{ Sample Description } \\
\hline $4 / 21 / 11$ & $11: 02$ & MRDIH2O-W-30059b & & & & & & & \\
\hline $4 / 21 / 11$ & 11:05 & MRQCTB-W-30061 ${ }^{b}$ & Water & TB & QC & - & 4826 & $4 / 21 / 11$ & $\begin{array}{l}\text { Trip blank sent to the AGEM Laboratory for } \\
\text { organic analysis with water samples listed on } \\
\text { COCs } 4825 \text { and } 4826 \text {. }\end{array}$ \\
\hline $4 / 21 / 11$ & $11: 15$ & MRQCIR-W-30057b & Water & RI & QC & - & 4826 & $4 / 21 / 11$ & $\begin{array}{l}\text { Rinsate of decontaminated sampling line after } \\
\text { collection of sample MRMW4S-W-30042. }\end{array}$ \\
\hline $7 / 28 / 11$ & $10: 20$ & MR019-B-29945 & Veg & Branch & MR019 & - & 6616 & $8 / 1 / 11$ & Silver maple, up $8 \mathrm{ft}, 16 \mathrm{ft}$ out. \\
\hline $7 / 28 / 11$ & $10: 25$ & MR013-B-29946 & Veg & Branch & MR013 & - & 6616 & $8 / 1 / 11$ & $\begin{array}{l}\text { Willow, } 3 \mathrm{ft} \text { up, out } 12 \text { in. Heavy weeds. Soy on } \\
\text { both sides of creek. }\end{array}$ \\
\hline $7 / 28 / 11$ & $10: 30$ & MR006-B-29947 & Veg & Branch & MR006 & - & 6616 & $8 / 1 / 11$ & Up $6 \mathrm{ft}$, out $2 \mathrm{ft}$. \\
\hline $7 / 28 / 11$ & $10: 34$ & MR007-B-29948 & Veg & Branch & MR007 & - & 6616 & $8 / 1 / 11$ & Up $4 \mathrm{ft}$. \\
\hline $7 / 28 / 11$ & 10:36 & MR008-B-29950 & Veg & Branch & MR008 & - & 6616 & $8 / 1 / 11$ & Up $5 \mathrm{ft}$, sucker. \\
\hline $7 / 28 / 11$ & 10:39 & MR014-B-29951 & Veg & Branch & MR014 & - & 6616 & $8 / 1 / 11$ & Up $4 \mathrm{ft}$, sucker. \\
\hline $7 / 28 / 11$ & $10: 42$ & MR009-B-29952 & Veg & Branch & MR009 & - & 6616 & $8 / 1 / 11$ & Up 4 ft, sucker. \\
\hline $7 / 28 / 11$ & $10: 44$ & MR010-B-29953 & Veg & Branch & MR010 & - & 6616 & $8 / 1 / 11$ & Up 6 ft, sucker. \\
\hline $7 / 28 / 11$ & $10: 46$ & MR011-B-29954 & Veg & Branch & MR011 & - & 6616 & $8 / 1 / 11$ & Up 4 ft, sucker. \\
\hline $7 / 28 / 11$ & $10: 49$ & MR012-B-29955 & Veg & Branch & MR012 & - & 6616 & $8 / 1 / 11$ & Over $16 \mathrm{ft}$ up, hard to tell. \\
\hline $7 / 28 / 11$ & $10: 51$ & MR016-B-29956 & Veg & Branch & MR016 & - & 6616 & $8 / 1 / 11$ & Up 4 ft, sucker. \\
\hline $7 / 28 / 11$ & $10: 53$ & MR017-B-29957 & Veg & Branch & MR017 & - & 6616 & $8 / 1 / 11$ & Up 3 ft, sucker. \\
\hline $7 / 28 / 11$ & $10: 55$ & MR018-B-29958 & Veg & Branch & MR018 & - & 6616 & $8 / 1 / 11$ & Up $6 \mathrm{ft}$, over $16 \mathrm{ft}$ out. \\
\hline $7 / 28 / 11$ & $11: 10$ & MR005A-B-29959 & Veg & Branch & MR005A & - & 6616 & $8 / 1 / 11$ & $\begin{array}{l}\text { Up } 5 \mathrm{ft} \text {, sucker. Storm damage, broken tops. } \\
\text { Near existing trash fire. }\end{array}$ \\
\hline $7 / 28 / 11$ & $11: 11$ & MR004-B-29960 & Veg & Branch & MR004 & - & 6616 & $8 / 1 / 11$ & $\begin{array}{l}\text { Willow, } 5 \mathrm{ft} \text { up, sucker. Tree has bent/cracked } \\
\text { top, down to ground. }\end{array}$ \\
\hline $7 / 28 / 11$ & $11: 12$ & MR003-B-29961 & Veg & Branch & MR003 & - & 6620 & $8 / 1 / 11$ & Up $5 \mathrm{ft}$, sucker. \\
\hline $7 / 28 / 11$ & $11: 14$ & MR002-B-29962 & Veg & Branch & MR002 & - & 6620 & $8 / 1 / 11$ & Up $5 \mathrm{ft}$, sucker. \\
\hline $7 / 28 / 11$ & $11: 17$ & MR001-B-29963 & Veg & Branch & MR001 & - & 6620 & $8 / 1 / 11$ & $\begin{array}{l}\text { Up } 5 \mathrm{ft} \text {, sucker. Behind new large stockpile of } \\
\text { trash to burn (wood shingles, tires, rotten } \\
\text { grain, misc. building materials). }\end{array}$ \\
\hline $7 / 28 / 11$ & $11: 20$ & MR038-B-29964 & Veg & Branch & MR038 & - & 6620 & $8 / 1 / 11$ & Up $4 \mathrm{ft}$, sucker. \\
\hline $7 / 28 / 11$ & $11: 22$ & MR039-B-29965 & Veg & Branch & MR039 & - & 6620 & $8 / 1 / 11$ & Up 4 ft, sucker. \\
\hline $7 / 28 / 11$ & $11: 24$ & MR040-B-29966 & Veg & Branch & MR040 & - & 6620 & $8 / 1 / 11$ & Up $5 \mathrm{ft}, 5 \mathrm{ft}$ out. \\
\hline $7 / 28 / 11$ & $12: 26$ & MR021-B-29967 & Veg & Branch & MR021 & - & 6620 & $8 / 1 / 11$ & Up 4 ft, 4 ft out. \\
\hline $7 / 28 / 11$ & $12: 30$ & MR025-B-29968 & Veg & Branch & MR025 & - & 6620 & $8 / 1 / 11$ & Up $4 \mathrm{ft}, 6 \mathrm{ft}$ out. \\
\hline $7 / 28 / 11$ & $12: 32$ & MR026-B-29969 & Veg & Branch & MR026 & - & 6620 & $8 / 1 / 11$ & Up 5 ft, sucker. \\
\hline $7 / 28 / 11$ & $12: 34$ & MR027-B-29970 & Veg & Branch & MR027 & - & 6620 & $8 / 1 / 11$ & Up 6 ft, sucker. \\
\hline $7 / 28 / 11$ & $12: 37$ & MR028-B-29971 & Veg & Branch & MR028 & - & 6620 & $8 / 1 / 11$ & Up 6 ft, sucker. \\
\hline
\end{tabular}




\begin{tabular}{|c|c|c|c|c|c|c|c|c|c|}
\hline \multicolumn{2}{|c|}{$\begin{array}{c}\text { Sample } \\
\text { Date and Time }\end{array}$} & Sample & \multirow{2}{*}{$\begin{array}{l}\text { Medium }^{\mathrm{a}} \\
\text { Veg }\end{array}$} & \multirow{2}{*}{$\begin{array}{l}\text { Type }^{\mathrm{a}} \\
\text { Branch }\end{array}$} & \multirow{2}{*}{$\begin{array}{l}\text { Location } \\
\text { MR029 }\end{array}$} & \multirow{2}{*}{$\begin{array}{c}\text { Depth } \\
\text { (ft BGL) } \\
\\
-\end{array}$} & \multirow{2}{*}{$\begin{array}{c}\text { Chain of } \\
\text { Custody }\end{array}$} & \multirow{2}{*}{$\begin{array}{c}\begin{array}{c}\text { Shipping } \\
\text { Date }\end{array} \\
8 / 1 / 11\end{array}$} & \multirow[t]{2}{*}{ Sample Description } \\
\hline $7 / 28 / 11$ & $12: 39$ & MR029-B-29972 & & & & & & & \\
\hline $7 / 28 / 11$ & $12: 41$ & MR030-B-29973 & Veg & Branch & MR030 & - & 6620 & $8 / 1 / 11$ & Up 6 ft, sucker. \\
\hline $7 / 28 / 11$ & $12: 44$ & MR031-B-29974 & Veg & Branch & MR031 & - & 6620 & $8 / 1 / 11$ & Up $7 \mathrm{ft}, 3 \mathrm{ft}$ out. \\
\hline $7 / 28 / 11$ & $12: 47$ & MR032-B-29975 & Veg & Branch & MR032 & - & 6620 & $8 / 1 / 11$ & Up $7 \mathrm{ft}$, sucker. \\
\hline $7 / 28 / 11$ & $12: 49$ & MR033-B-29976 & Veg & Branch & MR033 & - & 6621 & $8 / 1 / 11$ & Up $7 \mathrm{ft}, 2 \mathrm{ft}$ out. \\
\hline $7 / 28 / 11$ & $12: 51$ & MR034-B-29977 & Veg & Branch & MR034 & - & 6621 & $8 / 1 / 11$ & Up $6 \mathrm{ft}$, sucker. \\
\hline $7 / 28 / 11$ & $12: 56$ & MR037-B-29978 & Veg & Branch & MR037 & - & 6621 & $8 / 1 / 11$ & Up 4 ft, 3 ft out. \\
\hline $7 / 28 / 11$ & $12: 58$ & MR035-B-29979 & Veg & Branch & MR035 & - & 6621 & $8 / 1 / 11$ & Up $6 \mathrm{ft}$, sucker. \\
\hline $7 / 28 / 11$ & $13: 02$ & MR036-B-29980 & Veg & Branch & MR036 & - & 6621 & $8 / 1 / 11$ & Up $8 \mathrm{ft}, 4 \mathrm{ft}$ out. \\
\hline $7 / 28 / 11$ & $13: 03$ & MR024-B-29981 & Veg & Branch & MR024 & - & 6621 & $8 / 1 / 11$ & Up $4 \mathrm{ft}, 2 \mathrm{ft}$ out. \\
\hline $7 / 28 / 11$ & $13: 07$ & MR020-B-29982 & Veg & Branch & MR020 & - & 6621 & $8 / 1 / 11$ & Up $7 \mathrm{ft}, 2 \mathrm{ft}$ out. \\
\hline $7 / 28 / 11$ & $13: 10$ & MR043-B-29983 & Veg & Branch & MR043 & - & 6621 & $8 / 1 / 11$ & Up $3 \mathrm{ft}, 2 \mathrm{ft}$ out. \\
\hline $7 / 28 / 11$ & $13: 12$ & MR044-B-29984 & Veg & Branch & MR044 & - & 6621 & $8 / 1 / 11$ & Up $6 \mathrm{ft}, 3 \mathrm{ft}$ out. \\
\hline $7 / 28 / 11$ & $13: 15$ & MR041-B-29985 & Veg & Branch & MR041 & - & 6621 & $8 / 1 / 11$ & Up 4 ft, sucker. \\
\hline $7 / 28 / 11$ & $13: 17$ & MR042-B-29986 & Veg & Branch & MR042 & - & 6621 & $8 / 1 / 11$ & Up $3 \mathrm{ft}$. \\
\hline $10 / 3 / 11$ & $14: 08$ & MRSM4-W-30065 & Water & $\mathrm{N}$ & SM4 & - & 3188 & $10 / 4 / 11$ & Water sample from Terrapin Creek. \\
\hline $10 / 3 / 11$ & $14: 09$ & MRSM4-S-30065 & Sediment & $\mathrm{N}$ & SM4 & - & 3187 & $10 / 4 / 11$ & Sediment sample from Terrapin Creek. \\
\hline $10 / 3 / 11$ & $14: 20$ & MRSM3-W-30064 & Water & $\mathrm{N}$ & SM3 & - & 3188 & $10 / 4 / 11$ & Water sample from Terrapin Creek. \\
\hline $10 / 3 / 11$ & $14: 21$ & MRSM3-S-30064 & Sediment & $\mathrm{N}$ & SM3 & - & 3187 & $10 / 4 / 11$ & Sediment sample from Terrapin Creek. \\
\hline $10 / 3 / 11$ & $14: 30$ & MRSM2-W-30063 & Water & $\mathrm{N}$ & SM2 & - & 3188 & $10 / 4 / 11$ & Water sample from Terrapin Creek. \\
\hline $10 / 3 / 11$ & $14: 31$ & MRSM2-S-30063 & Sediment & $\mathrm{N}$ & SM2 & - & 3187 & $10 / 4 / 11$ & Sediment sample from Terrapin Creek. \\
\hline $10 / 3 / 11$ & $14: 40$ & MRSM1-W-30062 & Water & $\mathrm{N}$ & SM1 & - & 3188 & $10 / 4 / 11$ & Water sample from Terrapin Creek. \\
\hline $10 / 3 / 11$ & $14: 41$ & MRSM1-S-30062 & Sediment & $\mathrm{N}$ & SM1 & - & 3187 & $10 / 4 / 11$ & Sediment sample from Terrapin Creek. \\
\hline $10 / 3 / 11$ & $14: 50$ & MRSMB-W-30066 & Water & $\mathrm{N}$ & SMB & - & 3188 & $10 / 4 / 11$ & Water sample from Terrapin Creek. \\
\hline $10 / 3 / 11$ & $14: 51$ & MRSMB-S-30066 & Sediment & $\mathrm{N}$ & SMB & - & 3187 & $10 / 4 / 11$ & Sediment sample from Terrapin Creek. \\
\hline $10 / 3 / 11$ & $14: 58$ & MRTD12-W-30082 & Water & DW & TD12 & $27-67$ & 3188 & $10 / 4 / 11$ & $\begin{array}{l}\text { Collected from Grimm well overflow before the } \\
\text { catch pond. }\end{array}$ \\
\hline $10 / 3 / 11$ & $15: 14$ & MRStone-W-30081 & Water & DW & Stone & - & 3188 & $10 / 4 / 11$ & Bailed 5 gal before sampling. \\
\hline $10 / 3 / 11$ & $15: 24$ & MRRillinger-W-30080 & Water & DW & Rillinger & - & 3188 & $10 / 4 / 11$ & $\begin{array}{l}\text { Allowed pump to run for } 5 \text { min before collecting a } \\
\text { grab sample. }\end{array}$ \\
\hline $10 / 3 / 11$ & $16: 14$ & MRIsch-W-30079 & Water & DW & Isch & - & 3188 & $10 / 4 / 11$ & $\begin{array}{l}\text { Allowed pump to run for } 5 \text { min before collecting a } \\
\text { grab sample. Co-op has not used well in a few } \\
\text { weeks. }\end{array}$ \\
\hline $10 / 4 / 11$ & $9: 16$ & MRMW1D-W-30068 & Water & MW & MW1D & $63-88$ & 3190 & $10 / 4 / 11$ & $\begin{array}{l}\text { Depth to water }=29.06 \mathrm{ft} \text {. Depth of } 4 \text {-in. well = } \\
89 \mathrm{ft} \text {. Sample collected by using low-flow } \\
\text { bladder pump after purging of } 6.5 \mathrm{~L} \text {. Pump } \\
\text { intake positioned at } 75.5 \mathrm{ft} .\end{array}$ \\
\hline $10 / 4 / 11$ & $9: 17$ & MRMW1DDUP-W-30084 & Water & MW & MW1D & $63-88$ & 3190 & $10 / 4 / 11$ & Replicate of sample MRMW1D-W-30068. \\
\hline
\end{tabular}




\begin{tabular}{|c|c|c|c|c|c|c|c|c|c|}
\hline $\begin{array}{l}\text { Sam } \\
\text { Date an }\end{array}$ & $\begin{array}{l}\text { ple } \\
\text { Time }\end{array}$ & Sample & Medium $^{\mathrm{a}}$ & Type $^{a}$ & Location & $\begin{array}{l}\text { Depth } \\
\text { (ft BGL) }\end{array}$ & $\begin{array}{l}\text { Chain of } \\
\text { Custody }\end{array}$ & $\begin{array}{l}\text { Shipping } \\
\text { Date }\end{array}$ & Sample Description \\
\hline $10 / 4 / 11$ & 9:17 & MRMW8S-W-30075 & Water & MW & MW8S & $10-25$ & 3188 & $10 / 4 / 11$ & $\begin{array}{l}\text { Depth to water }=4.91 \mathrm{ft} \text {. Depth of } 4 \text {-in. well = } \\
26.8 \mathrm{ft} \text {. Sample collected by using low-flow } \\
\text { bladder pump after purging of } 9 \mathrm{~L} . \text { Pump } \\
\text { intake positioned at } 17.5 \mathrm{ft} \text {. }\end{array}$ \\
\hline $10 / 4 / 11$ & $10: 20$ & MRMW10S-W-30077 & Water & MW & MW10S & $30-45$ & 3188 & $10 / 4 / 11$ & $\begin{array}{l}\text { Depth to water }=12.54 \mathrm{ft} \text {. Depth of } 2 \text {-in. well = } \\
49.32 \mathrm{ft} \text {. Sample collected by using low-flow } \\
\text { bladder pump after purging of } 8 \mathrm{~L} \text {. Pump } \\
\text { intake positioned at } 37.5 \mathrm{ft} \text {. }\end{array}$ \\
\hline $10 / 4 / 11$ & $10: 24$ & MRMW1S-W-30067 & Water & MW & MW1S & $11-51$ & 3190 & $10 / 4 / 11$ & $\begin{array}{l}\text { Depth to water }=21.80 \mathrm{ft} \text {. Depth of } 4 \text {-in. well }= \\
54 \mathrm{ft} \text {. Sample collected by using low-flow } \\
\text { bladder pump after purging of } 8 \mathrm{~L} \text {. Pump } \\
\text { intake positioned at } 34.90 \mathrm{ft} .\end{array}$ \\
\hline $10 / 4 / 11$ & $10: 35$ & MRQCIR-W-30086 & Water & RI & QC & - & 3190 & $10 / 4 / 11$ & $\begin{array}{l}\text { Rinsate of decontaminated sampling line after } \\
\text { collection of sample MRMW1S-W-30067. }\end{array}$ \\
\hline $10 / 4 / 11$ & $11: 21$ & MRMW11S-W-30078 & Water & MW & MW11S & $53-68$ & 3188 & $10 / 4 / 11$ & $\begin{array}{l}\text { Depth to water }=36.83 \mathrm{ft} \text {. Depth of } 2 \text {-in. well = } \\
72.7 \mathrm{ft} \text {. Sample collected by using low-flow } \\
\text { bladder pump after purging of } 6 \mathrm{~L} \text {. Pump } \\
\text { intake positioned at } 60.5 \mathrm{ft} \text {. }\end{array}$ \\
\hline $10 / 4 / 11$ & $11: 28$ & MRMW2S-W-30069 & Water & MW & MW2S & $13-53$ & 3190 & $10 / 4 / 11$ & $\begin{array}{l}\text { Depth to water }=32.73 \mathrm{ft} \text {. Depth of } 4 \text {-in. well = } \\
53.5 \mathrm{ft} \text {. Sample collected by using low-flow } \\
\text { bladder pump after purging of } 7.5 \mathrm{~L} \text {. Pump } \\
\text { intake positioned at } 42.6 \mathrm{ft} \text {. }\end{array}$ \\
\hline $10 / 4 / 11$ & $12: 22$ & MRMW3S-W-30070 & Water & MW & MW3S & $18-48$ & 3190 & $10 / 4 / 11$ & $\begin{array}{l}\text { Depth to water }=25.95 \mathrm{ft} \text {. Depth of } 4 \text {-in. well }= \\
47.8 \mathrm{ft} \text {. Sample collected by using low-flow } \\
\text { bladder pump after purging of } 7 \mathrm{~L} \text {. Pump } \\
\text { intake positioned at } 37 \mathrm{ft} \text {. }\end{array}$ \\
\hline $10 / 4 / 11$ & $12: 32$ & MRMW7S-W-30074 & Water & MW & MW7S & $20-45$ & 3188 & $10 / 4 / 11$ & $\begin{array}{l}\text { Depth to water = } 7.11 \mathrm{ft} \text {. Depth of } 4 \text {-in. well = } \\
47 \mathrm{ft} \text {. Sample collected by using low-flow } \\
\text { bladder pump after purging of } 6 \mathrm{~L} \text {. Pump } \\
\text { intake positioned at } 32.5 \mathrm{ft} \text {. }\end{array}$ \\
\hline $10 / 4 / 11$ & $12: 33$ & MRMW7SDUP-W-30083 ${ }^{b}$ & Water & MW & MW7S & $20-45$ & 3190 & $10 / 4 / 11$ & Replicate of sample MRMW7S-W-30074. \\
\hline $10 / 4 / 11$ & $12: 55$ & MRQCIR-W-30085 & Water & $\mathrm{RI}$ & QC & - & 3188 & $10 / 4 / 11$ & $\begin{array}{l}\text { Rinsate of decontaminated sampling line after } \\
\text { collection of sample MRMW7S-W-30074 and } \\
\text { replicate MRMW7SDUP-W-30083. }\end{array}$ \\
\hline $10 / 4 / 11$ & $13: 00$ & MRQCTB-W-30089b & Water & TB & QC & - & 3188 & $10 / 4 / 11$ & $\begin{array}{l}\text { Trip blank sent to the AGEM Laboratory for } \\
\text { VOCs analysis with water samples listed on } \\
\text { COC } 3188 \text {. }\end{array}$ \\
\hline
\end{tabular}


TABLE A.1 (Cont.)

\begin{tabular}{|c|c|c|c|c|c|c|c|c|c|}
\hline \multicolumn{2}{|c|}{$\begin{array}{c}\text { Sample } \\
\text { Date and Time }\end{array}$} & Sample & Medium $^{\mathrm{a}}$ & Type $^{a}$ & Location & $\begin{array}{l}\text { Depth } \\
\text { (ft BGL) }\end{array}$ & $\begin{array}{l}\text { Chain of } \\
\text { Custody }\end{array}$ & $\begin{array}{l}\text { Shipping } \\
\text { Date }\end{array}$ & Sample Description \\
\hline $10 / 4 / 11$ & $13: 26$ & MRMW4S-W-30071 & Water & MW & MW4S & $17-47$ & 3190 & $10 / 4 / 11$ & $\begin{array}{l}\text { Depth to water }=35.60 \mathrm{ft} \text {. Depth of } 4 \text {-in. well = } \\
47.8 \mathrm{ft} \text {. Sample collected by using low-flow } \\
\text { bladder pump after purging of } 5 \mathrm{~L} \text {. Pump } \\
\text { intake positioned at } 40.9 \mathrm{ft} \text {. }\end{array}$ \\
\hline $10 / 4 / 11$ & $14: 22$ & MRMW9S-W-30076 & Water & MW & MW9S & $\begin{array}{l}38.83- \\
53.83\end{array}$ & 3190 & $10 / 4 / 11$ & $\begin{array}{l}\text { Depth to water }=22.35 \mathrm{ft} \text {. Depth of } 2 \text {-in. well = } \\
58.5 \mathrm{ft} \text {. Sample collected by using low-flow } \\
\text { bladder pump after purging of } 8 \mathrm{~L} \text {. Pump } \\
\text { intake positioned at } 46.33 \mathrm{ft} \text {. }\end{array}$ \\
\hline $10 / 4 / 11$ & $14: 50$ & MRQCIR-W-30087 & Water & RI & QC & - & 3190 & $10 / 4 / 11$ & $\begin{array}{l}\text { Rinsate of decontaminated sampling line after } \\
\text { collection of sample MRMW9S-W-30076. }\end{array}$ \\
\hline $10 / 4 / 11$ & $15: 30$ & MRDIH2O-W-30088b & Water & FB & QC & - & 3190 & $10 / 4 / 11$ & $\begin{array}{l}\text { Field blank of water used for equipment } \\
\text { decontamination during September } 2011 \\
\text { monitoring. }\end{array}$ \\
\hline $10 / 4 / 11$ & $15: 30$ & MRQCTB-W-30090 b & Water & TB & QC & - & 3190 & $10 / 4 / 11$ & $\begin{array}{l}\text { Trip blank sent to the AGEM Laboratory for } \\
\text { VOCs analysis with water samples listed on } \\
\text { COC } 3190 \text {. }\end{array}$ \\
\hline $10 / 4 / 11$ & $15: 31$ & MRMW6S-W-30073 & Water & MW & MW6S & $10-25$ & 3190 & $10 / 4 / 11$ & $\begin{array}{l}\text { Depth to water }=6.49 \mathrm{ft} \text {. Depth of } 4 \text {-in. well = } \\
26.9 \mathrm{ft} \text {. Sample collected by using low-flow } \\
\text { bladder pump after purging of } 6 \mathrm{~L} \text {. Pump } \\
\text { intake positioned at } 17.5 \mathrm{ft} \text {. }\end{array}$ \\
\hline $10 / 4 / 11$ & $17: 18$ & MRMW5S-W-30072 & Water & MW & MW5S & $15-55$ & 3190 & $10 / 4 / 11$ & $\begin{array}{l}\text { Depth to water }=21.10 \mathrm{ft} \text {. Depth of } 4 \text {-in. well = } \\
54.6 \mathrm{ft} \text {. Sample collected by using low-flow } \\
\text { bladder pump after purging of } 6.5 \mathrm{~L} \text {. Pump } \\
\text { intake positioned at } 38.25 \mathrm{ft} \text {. }\end{array}$ \\
\hline $10 / 12 / 11$ & $14: 34$ & MRMW1S-W-30091 & Water & MW & MW1S & $11-51$ & 2772 & $10 / 12 / 11$ & $\begin{array}{l}\text { Grab sample for sampling method comparison. } \\
\text { Depth to water }=22.14 \mathrm{ft} \text {. Depth of } 4 \text {-in. well = } \\
54 \mathrm{ft} \text {. Sample collected by using low-flow } \\
\text { bladder pump after purging of } 6 \mathrm{~L} \text {. Pump } \\
\text { intake positioned at } 36.5 \mathrm{ft} \text {. }\end{array}$ \\
\hline $10 / 12 / 11$ & $15: 23$ & MRMW1S3X-W-30092 & Water & MW & MW1S & $11-51$ & 2772 & $10 / 12 / 11$ & $\begin{array}{l}\text { Sample collected after purging of three well } \\
\text { volumes. Pump intake at } 49 \mathrm{ft} .\end{array}$ \\
\hline $10 / 12 / 11$ & $17: 50$ & MRQCTB-W-30093 & Water & TB & QC & - & 2772 & $10 / 12 / 11$ & $\begin{array}{l}\text { Trip blank sent to the AGEM Laboratory for } \\
\text { VOCs analysis with water samples listed on } \\
\text { COC } 2772 \text {. }\end{array}$ \\
\hline
\end{tabular}


TABLE A.1 (Cont.)

Sample

Date and Time

Sample

Mediuma

Type

Location (ft BGL)

Chain of
Custody
Date

Custody

Sample Description

a Medium and sample type abbreviations: DW, domestic well; MW, monitoring well; RI, equipment rinsate; sed, sediment; SW, surface water; TB, trip blank; veg, vegetation.

b Quality control sample. 
Appendix B:

Results from the AGEM Laboratory for Dual Analyses of Samples Collected at Morrill in 2011 and for Quality Control Samples 
TABLE B.1 Analytical results from the AGEM Laboratory for quality control samples collected to monitor sample collection and handling activities in 2011.

\begin{tabular}{|c|c|c|c|c|c|}
\hline \multirow[b]{2}{*}{$\begin{array}{l}\text { Sample } \\
\text { Date }\end{array}$} & \multirow[b]{2}{*}{ Sample } & \multirow[b]{2}{*}{ Type } & \multicolumn{3}{|c|}{ Concentration $(\mu \mathrm{g} / \mathrm{L})$} \\
\hline & & & $\begin{array}{c}\text { Carbon } \\
\text { Tetrachloride }\end{array}$ & Chloroform & $\begin{array}{l}\text { Methylene } \\
\text { Chloride }\end{array}$ \\
\hline $4 / 20 / 2011$ & MRQCTB-W-30060 & Trip blank & $N D^{a}$ & ND & ND \\
\hline $4 / 20 / 2011$ & MRQCIR-W-30056 & Equipment rinsate & ND & ND & ND \\
\hline $4 / 20 / 2011$ & MRQCIR-W-30058 & Equipment rinsate & ND & ND & ND \\
\hline 4/21/2011 & MRDIH2O-W-30059 & Field blank & ND & ND & ND \\
\hline 4/21/2011 & MRQCTB-W-30061 & Trip blank & ND & ND & ND \\
\hline 4/21/2011 & MRQCIR-W-30057 & Equipment rinsate & ND & ND & ND \\
\hline 10/4/2011 & MRQCIR-W-30086 & Equipment rinsate & ND & ND & ND \\
\hline 10/4/2011 & MRQCIR-W-30085 & Equipment rinsate & ND & ND & ND \\
\hline $10 / 4 / 2011$ & MRQCTB-W-30089 & Trip blank & ND & ND & ND \\
\hline 10/4/2011 & MRQCIR-W-30087 & Equipment rinsate & ND & ND & ND \\
\hline 10/4/2011 & MRDIH2O-W-30088 & Field blank & ND & ND & ND \\
\hline $10 / 4 / 2011$ & MRQCTB-W-30090 & Trip blank & ND & ND & ND \\
\hline 10/12/2011 & MRQCTB-W-30093 & Trip blank & ND & ND & ND \\
\hline
\end{tabular}

a ND, contaminant not detected at an instrument detection limit of $0.1 \mu \mathrm{g} / \mathrm{L}$. 
TABLE B.2 Analytical results from the AGEM Laboratory for dual analyses of samples collected in 2011.

\begin{tabular}{|c|c|c|c|c|c|c|c|c|}
\hline \multirow[b]{2}{*}{$\begin{array}{l}\text { Sample } \\
\text { Date }\end{array}$} & \multirow[b]{2}{*}{ Location } & \multirow[b]{2}{*}{ Sample } & \multirow[b]{2}{*}{$\begin{array}{l}\text { Analysis } \\
\text { Type }\end{array}$} & \multirow[b]{2}{*}{$\begin{array}{c}\text { Depth } \\
\text { (ft BGL) }\end{array}$} & \multirow[b]{2}{*}{$\begin{array}{l}\text { Sample } \\
\text { Medium }\end{array}$} & \multicolumn{3}{|c|}{$\begin{array}{c}\text { Concentration } \\
(\mu \mathrm{g} / \mathrm{kg} \text { in soil; } \mu \mathrm{g} / \mathrm{L} \text { in water })\end{array}$} \\
\hline & & & & & & $\begin{array}{l}\text { Carbon } \\
\text { Tetrachloride }\end{array}$ & Chloroform & $\begin{array}{l}\text { Methylene } \\
\text { Chloride }\end{array}$ \\
\hline $4 / 20 / 2011$ & SM3 & MRSM3-S-30035 & Primary sample & - & Sediment & $N D^{a}$ & ND & ND \\
\hline $4 / 20 / 2011$ & SM3 & MRSM3-S-30035DUP & Duplicate analysis & - & Sediment & ND & ND & ND \\
\hline $4 / 20 / 2011$ & MW1S & MRMW1S-W-30038 & Primary sample & $11-51$ & Water & $0.3 \mathrm{~J}^{\mathrm{b}}$ & ND & ND \\
\hline $4 / 20 / 2011$ & MW1S & MRMW1S-W-30038DUP & Duplicate analysis & $11-51$ & Water & $0.3 \mathrm{~J}$ & ND & ND \\
\hline $\begin{array}{l}4 / 20 / 2011 \\
4 / 20 / 2011\end{array}$ & $\begin{array}{l}\text { MW3S } \\
\text { MW3S }\end{array}$ & $\begin{array}{l}\text { MRMW3S-W-30041 } \\
\text { MRMW3S-W-30041DUP }\end{array}$ & Primary sample & $\begin{array}{l}18-48 \\
18-48\end{array}$ & Water & $\begin{array}{l}33 \\
29\end{array}$ & 1.2 & ND \\
\hline $\begin{array}{l}4 / 20 / 2011 \\
4 / 20 / 2011\end{array}$ & $\begin{array}{l}\text { MW9S } \\
\text { MW9S }\end{array}$ & $\begin{array}{l}\text { MRMW9S-W-30047 } \\
\text { MRMW9SDUP-W-30054 }\end{array}$ & $\begin{array}{l}\text { Primary sample } \\
\text { Replicate sample }\end{array}$ & $\begin{array}{l}38.83-53.83 \\
38.83-53.83\end{array}$ & $\begin{array}{l}\text { Water } \\
\text { Water }\end{array}$ & $\begin{array}{l}0.9 \mathrm{~J} \\
1.1\end{array}$ & $\begin{array}{l}\text { ND } \\
\text { ND }\end{array}$ & $\begin{array}{l}\text { ND } \\
\text { ND }\end{array}$ \\
\hline $\begin{array}{l}4 / 20 / 2011 \\
4 / 20 / 2011\end{array}$ & $\begin{array}{l}\text { MW2S } \\
\text { MW2S }\end{array}$ & $\begin{array}{l}\text { MRMW2S-W-30040 } \\
\text { MRMW2SDUP-W-30055 }\end{array}$ & $\begin{array}{l}\text { Primary sample } \\
\text { Replicate sample }\end{array}$ & $\begin{array}{l}13-53 \\
13-53\end{array}$ & $\begin{array}{l}\text { Water } \\
\text { Water }\end{array}$ & $\begin{array}{l}\text { ND } \\
\text { ND }\end{array}$ & $\begin{array}{l}\text { ND } \\
\text { ND }\end{array}$ & $\begin{array}{l}\text { ND } \\
\text { ND }\end{array}$ \\
\hline $\begin{array}{l}10 / 3 / 2011 \\
10 / 3 / 2011\end{array}$ & $\begin{array}{l}\text { SM4 } \\
\text { SM4 }\end{array}$ & $\begin{array}{l}\text { MRSM4-S-30065 } \\
\text { MRSM4-S-30065DUP }\end{array}$ & $\begin{array}{l}\text { Primary sample } \\
\text { Duplicate analysis }\end{array}$ & $\begin{array}{l}- \\
-\end{array}$ & $\begin{array}{l}\text { Sediment } \\
\text { Sediment }\end{array}$ & $\begin{array}{l}\text { ND } \\
\text { ND }\end{array}$ & $\begin{array}{l}\text { ND } \\
\text { ND }\end{array}$ & $\begin{array}{l}\text { ND } \\
\text { ND }\end{array}$ \\
\hline $\begin{array}{l}10 / 4 / 2011 \\
10 / 4 / 2011\end{array}$ & $\begin{array}{l}\text { MW1D } \\
\text { MW1D }\end{array}$ & $\begin{array}{l}\text { MRMW1D-W-30068 } \\
\text { MRMW1DDUP-W-30084 }\end{array}$ & $\begin{array}{l}\text { Primary sample } \\
\text { Replicate sample }\end{array}$ & $\begin{array}{l}63-88 \\
63-88\end{array}$ & $\begin{array}{l}\text { Water } \\
\text { Water }\end{array}$ & $\begin{array}{l}\text { ND } \\
\text { ND }\end{array}$ & $\begin{array}{l}\text { ND } \\
\text { ND }\end{array}$ & $\begin{array}{l}\text { ND } \\
\text { ND }\end{array}$ \\
\hline $\begin{array}{l}10 / 4 / 2011 \\
10 / 4 / 2011\end{array}$ & $\begin{array}{l}\text { MW3S } \\
\text { MW3S }\end{array}$ & $\begin{array}{l}\text { MRMW3S-W-30070 } \\
\text { MRMW3S-W-30070DUP }\end{array}$ & $\begin{array}{l}\text { Primary sample } \\
\text { Duplicate analysis }\end{array}$ & $\begin{array}{l}18-48 \\
18-48\end{array}$ & $\begin{array}{l}\text { Water } \\
\text { Water }\end{array}$ & $\begin{array}{l}49 \\
48\end{array}$ & $\begin{array}{l}1.8 \\
1.8\end{array}$ & $\begin{array}{l}\text { ND } \\
\text { ND }\end{array}$ \\
\hline $\begin{array}{l}10 / 4 / 2011 \\
10 / 4 / 2011 \\
10 / 4 / 2011\end{array}$ & $\begin{array}{l}\text { MW7S } \\
\text { MW7S } \\
\text { MW7S }\end{array}$ & $\begin{array}{l}\text { MRMW7S-W-30074 } \\
\text { MRMW7S-W-30074DUP } \\
\text { MRMW7SDUP-W-30083 }\end{array}$ & $\begin{array}{l}\text { Primary sample } \\
\text { Duplicate analysis } \\
\text { Replicate sample }\end{array}$ & $\begin{array}{l}20-45 \\
20-45 \\
20-45\end{array}$ & $\begin{array}{l}\text { Water } \\
\text { Water } \\
\text { Water }\end{array}$ & $\begin{array}{l}11 \\
11 \\
12\end{array}$ & $\begin{array}{l}0.3 \\
0.3 \\
0.3\end{array}$ & $\begin{array}{l}\text { ND } \\
\text { ND } \\
\text { ND }\end{array}$ \\
\hline $\begin{array}{l}10 / 4 / 2011 \\
10 / 4 / 2011\end{array}$ & $\begin{array}{l}\text { MW9S } \\
\text { MW9S }\end{array}$ & $\begin{array}{l}\text { MRMW9S-W-30076 } \\
\text { MRMW9S-W-30076DUP }\end{array}$ & $\begin{array}{l}\text { Primary sample } \\
\text { Duplicate analysis }\end{array}$ & $\begin{array}{l}38.83-53.83 \\
38.83-53.83\end{array}$ & $\begin{array}{l}\text { Water } \\
\text { Water }\end{array}$ & $\begin{array}{l}0.9 \mathrm{~J} \\
0.8 \mathrm{~J}\end{array}$ & $\begin{array}{l}\text { ND } \\
\text { ND }\end{array}$ & $\begin{array}{l}\text { ND } \\
\text { ND }\end{array}$ \\
\hline $\begin{array}{l}10 / 12 / 2011 \\
10 / 12 / 2011\end{array}$ & $\begin{array}{l}\text { MW1S } \\
\text { MW1S }\end{array}$ & $\begin{array}{l}\text { MRMW1S3X-W-30092 } \\
\text { MRMW1S3X-W-30092DUP }\end{array}$ & $\begin{array}{l}\text { Primary sample } \\
\text { Duplicate analysis }\end{array}$ & $\begin{array}{l}11-51 \\
11-51\end{array}$ & $\begin{array}{l}\text { Water } \\
\text { Water }\end{array}$ & $\begin{array}{l}23 \\
25\end{array}$ & $\begin{array}{l}1.2 \\
1.4\end{array}$ & $\begin{array}{l}\text { ND } \\
\text { ND }\end{array}$ \\
\hline
\end{tabular}

a ND, contaminant not detected at an instrument detection limit of $0.1 \mu \mathrm{g} / \mathrm{L}$ for water analyses or $1.0 \mu \mathrm{g} / \mathrm{kg}$ for soil analyses.

b Qualifier J indicates an estimated concentration below the purge-and-trap method quantitation limit of $1.0 \mu \mathrm{g} / \mathrm{L}$. 
TABLE B.3 Analytical results from the AGEM Laboratory and TestAmerica for samples collected in 2011 and submitted for verification analysis.

\begin{tabular}{|c|c|c|c|c|c|c|c|c|c|}
\hline \multirow[b]{3}{*}{ Location } & \multirow[b]{3}{*}{ Sample } & \multirow[b]{3}{*}{$\begin{array}{l}\text { Screen } \\
\text { Interval } \\
\text { (ft BGL) }\end{array}$} & \multirow[b]{3}{*}{$\begin{array}{l}\text { Sample } \\
\text { Date }\end{array}$} & \multicolumn{6}{|c|}{ Concentration $(\mu \mathrm{g} / \mathrm{L})$} \\
\hline & & & & \multicolumn{3}{|c|}{ AGEM Laboratory } & \multicolumn{3}{|c|}{ TestAmerica } \\
\hline & & & & $\begin{array}{c}\text { Carbon } \\
\text { Tetrachloride }\end{array}$ & Chloroform & $\begin{array}{l}\text { Methylene } \\
\text { Chloride }\end{array}$ & $\begin{array}{c}\text { Carbon } \\
\text { Tetrachloride }\end{array}$ & Chloroform & $\begin{array}{l}\text { Methylene } \\
\text { Chloride }\end{array}$ \\
\hline \multicolumn{10}{|c|}{ April 2011 sampling event } \\
\hline MW1D & MRMW1D-W-30039 & $63-88$ & $4 / 20 / 11$ & $N D^{a}$ & ND & ND & $0.022 \mathrm{~J}^{\mathrm{b}} \mathrm{B}^{\mathrm{c}}$ & ND & ND \\
\hline MW6S & MRMW6S-W-30044 & $10-25$ & $4 / 20 / 11$ & ND & ND & ND & $0.035 \mathrm{~J} \mathrm{~B}$ & ND & ND \\
\hline MW4S & MRMW4S-W-30042 & $17-47$ & $4 / 21 / 11$ & ND & ND & ND & $0.15 \mathrm{~J} \mathrm{~B}$ & ND & ND \\
\hline Rillinger & MRRILLINGER-W-30051 & - & $4 / 21 / 11$ & $0.7 \mathrm{~J}$ & ND & ND & $0.39 \mathrm{~J} \mathrm{~B}$ & ND & ND \\
\hline QC & MRQCTB-W-30061 & - & $4 / 21 / 11$ & ND & ND & ND & $0.023 \mathrm{~J} \mathrm{~B}$ & ND & ND \\
\hline \multicolumn{10}{|c|}{ October 2011 sampling event } \\
\hline SM2 & MRSW2-W-30063 & - & $10 / 3 / 11$ & ND & ND & ND & $0.045 \mathrm{~J} \mathrm{~B}$ & ND & ND \\
\hline MW2S & MRMW2S-W-30069 & $13-53$ & $10 / 4 / 11$ & ND & ND & ND & $0.043 \mathrm{~J} \mathrm{~B}$ & ND & ND \\
\hline MW4S & MRMW4S-W-30071 & $17-47$ & $10 / 4 / 11$ & ND & ND & ND & $0.11 \mathrm{~J} \mathrm{~B}$ & ND & ND \\
\hline MW11S & MRMW11S-W-30078 & $53-68$ & $10 / 4 / 11$ & 33 & $0.8 \mathrm{~J}$ & ND & 35 & 0.85 & ND \\
\hline QC & MRQCTB-W-30090 & - & $10 / 4 / 11$ & ND & ND & ND & $0.016 \mathrm{~J} \mathrm{~B}$ & ND & ND \\
\hline
\end{tabular}

a ND, not detected at instrument detection limit of $0.1 \mu \mathrm{g} / \mathrm{L}$ for analyses by the AGEM Laboratory or $0.01 \mu \mathrm{g} / \mathrm{L}$ for analyses by TestAmerica.

b Qualifier J indicates an estimated concentration below the method quantitation limit of $1.0 \mu \mathrm{g} / \mathrm{L}$ for analyses by the AGEM Laboratatory or $0.5 \mu \mathrm{g} / \mathrm{L}$ for analyses by TestAmerica.

c Qualifier B indicates the presence of the contaminant in associated laboratory method blanks and/or trip blanks. 
Supplement 1:

Waste Characterization Data 
Pace Analytical Services, Inc. 9608 Loiret Blvd. Lenexa, KS 66219 (913)599-5665

November 11, 2011

Mr. Travis Kamler

TCW Construction Inc

$141 \mathrm{M}$ Street

Lincoln, NE 68508

RE: Project: KS/MO Waste Water

Pace Project No.: 60109211

Dear Mr. Kamler:

Enclosed are the analytical results for sample(s) received by the laboratory on November 01, 2011.

The results relate only to the samples included in this report. Results reported herein conform to the most current TNI standards and the laboratory's Quality Assurance Manual, where applicable, unless otherwise noted in the body of the report.

If you have any questions concerning this report, please feel free to contact me.

Sincerely,

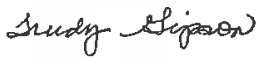

Trudy Gipson

trudy.gipson@pacelabs.com

Project Manager

Enclosures

cc: Mr. David Surgnier

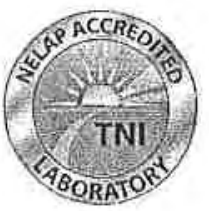




\section{CERTIFICATIONS}

Project: $\quad$ KS/MO Waste Water

Pace Project No.: 60109211

Kansas Certification IDs

9608 Loiret Boulevard, Lenexa, KS 66219

A2LA Certification \#: 2456.01

Arkansas Certification \#: 05-008-0

Illinois Certification \#: 001191

lowa Certification \#: 118

Kansas/NELAP Certificalion \#: E-10116
Louisiana Certification \#: 03055

Nevada Cerlification \#: KS000212008A

Oklahoma Certification \#: 9205/9935

Texas Certification \#: T104704407-08-TX

Utah Certification \#: 9135995665 


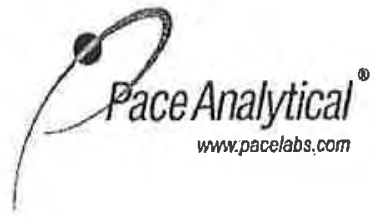

Pace Analytical Services, Inc. 9608 Loiret Blvd. Lenexa, KS 66219 (913)599-5665

SAMPLE SUMMARY

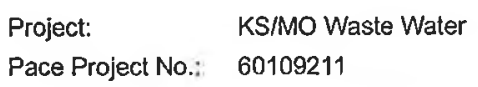

\begin{tabular}{|c|c|c|c|c|}
\hline Lab ID & Sample ID & Matrix & Date Collected & Date Received \\
\hline 60109211001 & AGPURGE-W-10111 & Water & $10 / 31 / 1109: 00$ & $11 / 01 / 1109: 20$ \\
\hline 60109211002 & BAPURGE-W-10112 & Water & $10 / 31 / 1112: 55$ & $11 / 01 / 1109: 20$ \\
\hline 60109211003 & CNPURGE-W-10113 & Water & $10 / 31 / 1114: 02$ & 11/01/11 09:20 \\
\hline 60109211004 & EUPURGE-W-10114 & Water & 10/31/11 15:52 & $11 / 01 / 1109: 20$ \\
\hline 60109211005 & HAPURGE-W-10115 & Water & $10 / 31 / 1112: 27$ & $11 / 01 / 1109: 20$ \\
\hline 60109211006 & MRPURGE-W-10116 & Water & $10 / 31 / 1114: 42$ & $11 / 01 / 1109: 20$ \\
\hline 60109211007 & SVPURGE-W-10117 & Water & $10 / 31 / 11 \quad 18: 30$ & $11 / 01 / 1109: 20$ \\
\hline
\end{tabular}




\section{SAMPLE ANALYTE COUNT}

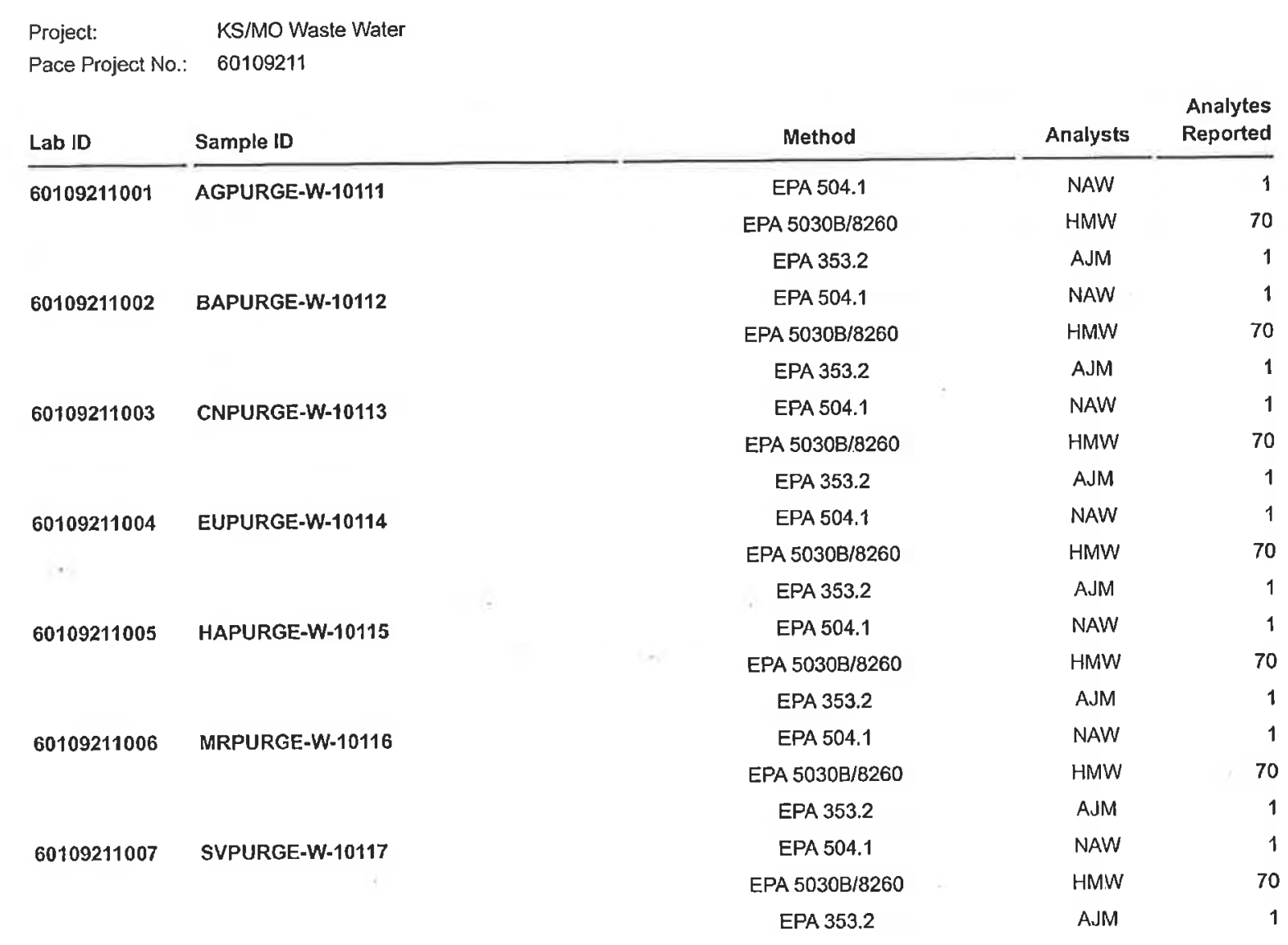




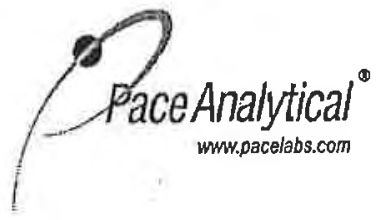

Pace Analytical Services, Inc. 9608 Loiret Blvd. Lenexa, KS 66219

(913)599-5665

\section{ANALYTICAL RESULTS}

Project:

KS/MO Waste Water

Pace Project No.: $\quad 60109211$

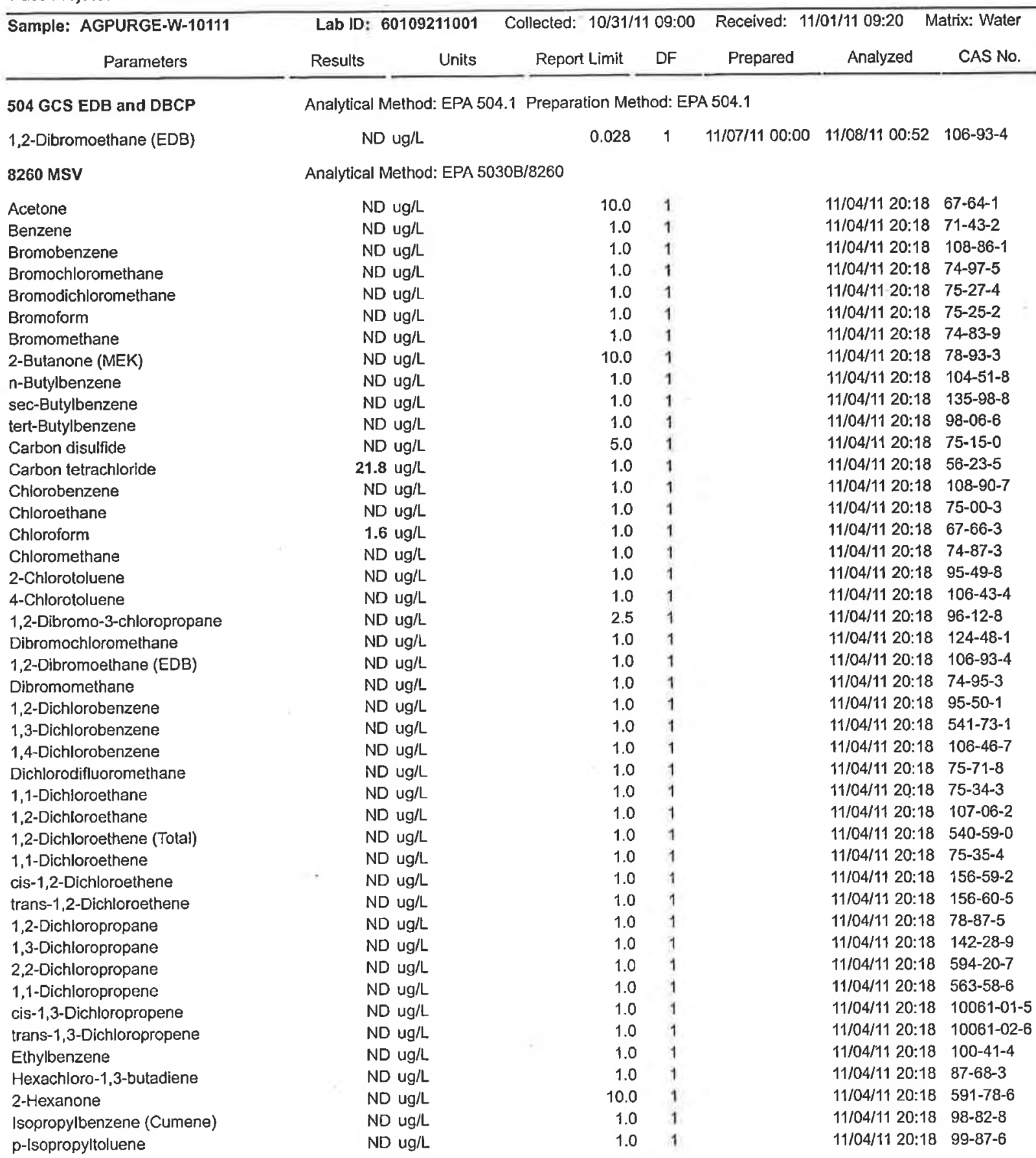

REPORT OF LABORATORY ANALYSIS

Page 5 of 26

Date: 11/11/2011 11:15 AM

This report shall not be reproduced, except in full,

without the written consent of Pace Analytical Services, Inc. 


\section{ANALYTICAL RESULTS}

Project: $\quad$ KS/MO Waste Water

Pace Project No.: 60109211

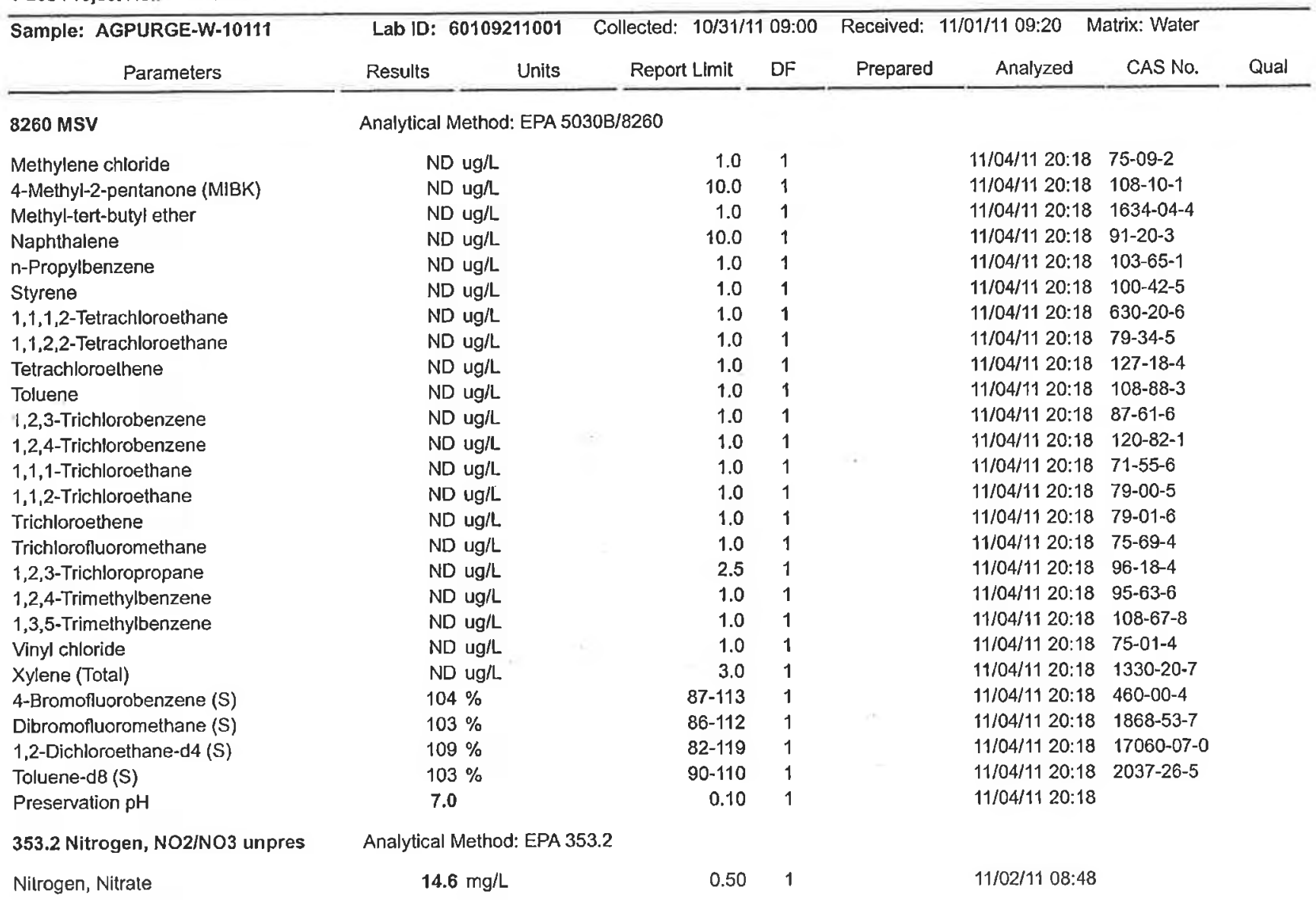




\section{ANALYTICAL RESULTS}

Project: KS/MO Waste Water

Pace Project No.: 60109211

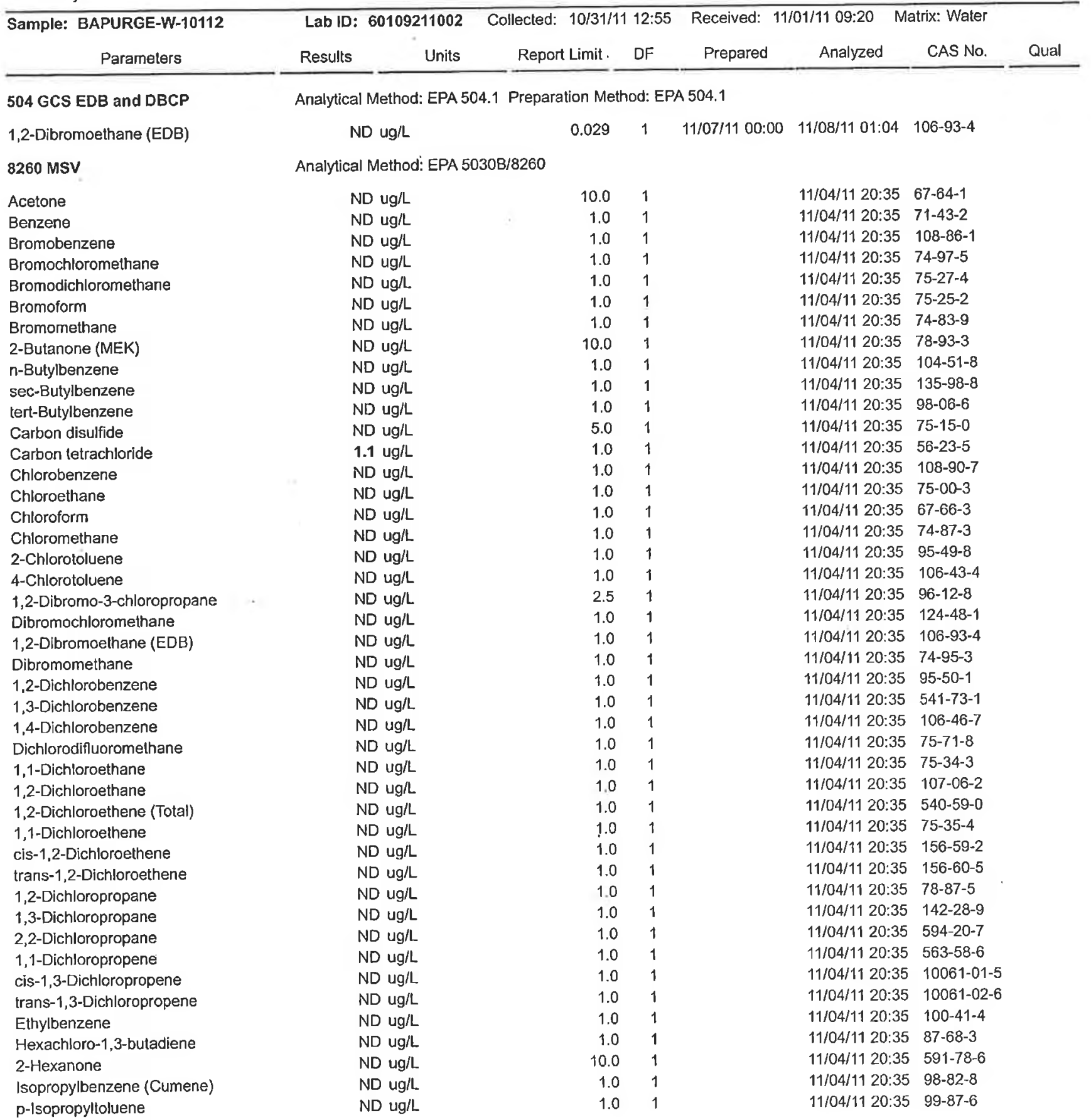

REPORT OF LABORATORY ANALYSIS 
Pace Analytical Services, Inc. 9608 Loiret Blvd. Lenexa, KS 66219

(913)599-5665

\section{ANALYTICAL RESULTS}

Project:

KS/MO Waste Water

Pace Project No.: $\quad 60109211$

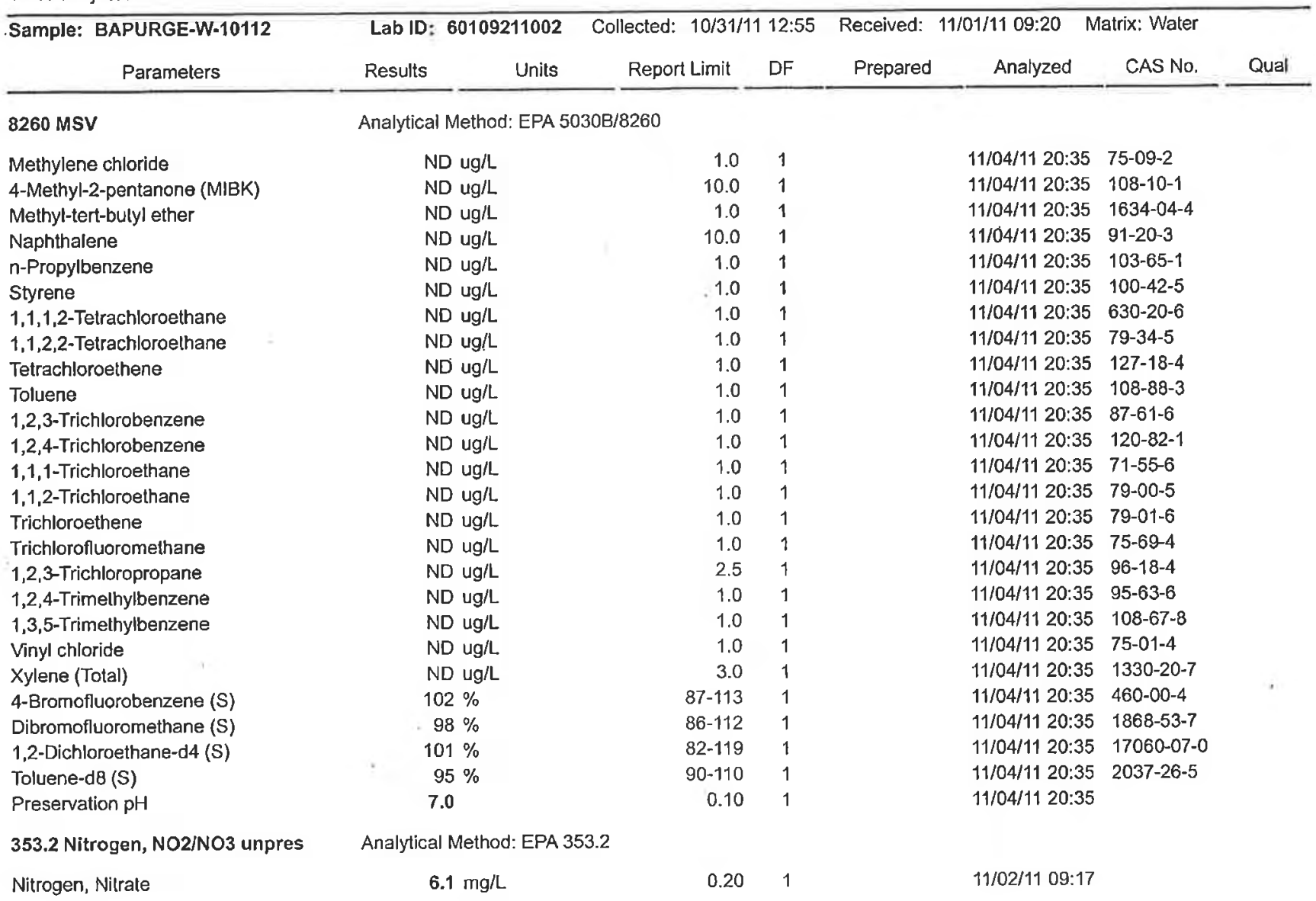


ANALYTICAL RESULTS

Project: $\quad$ KS/MO Waste Water

Pace Project No.: 60109211

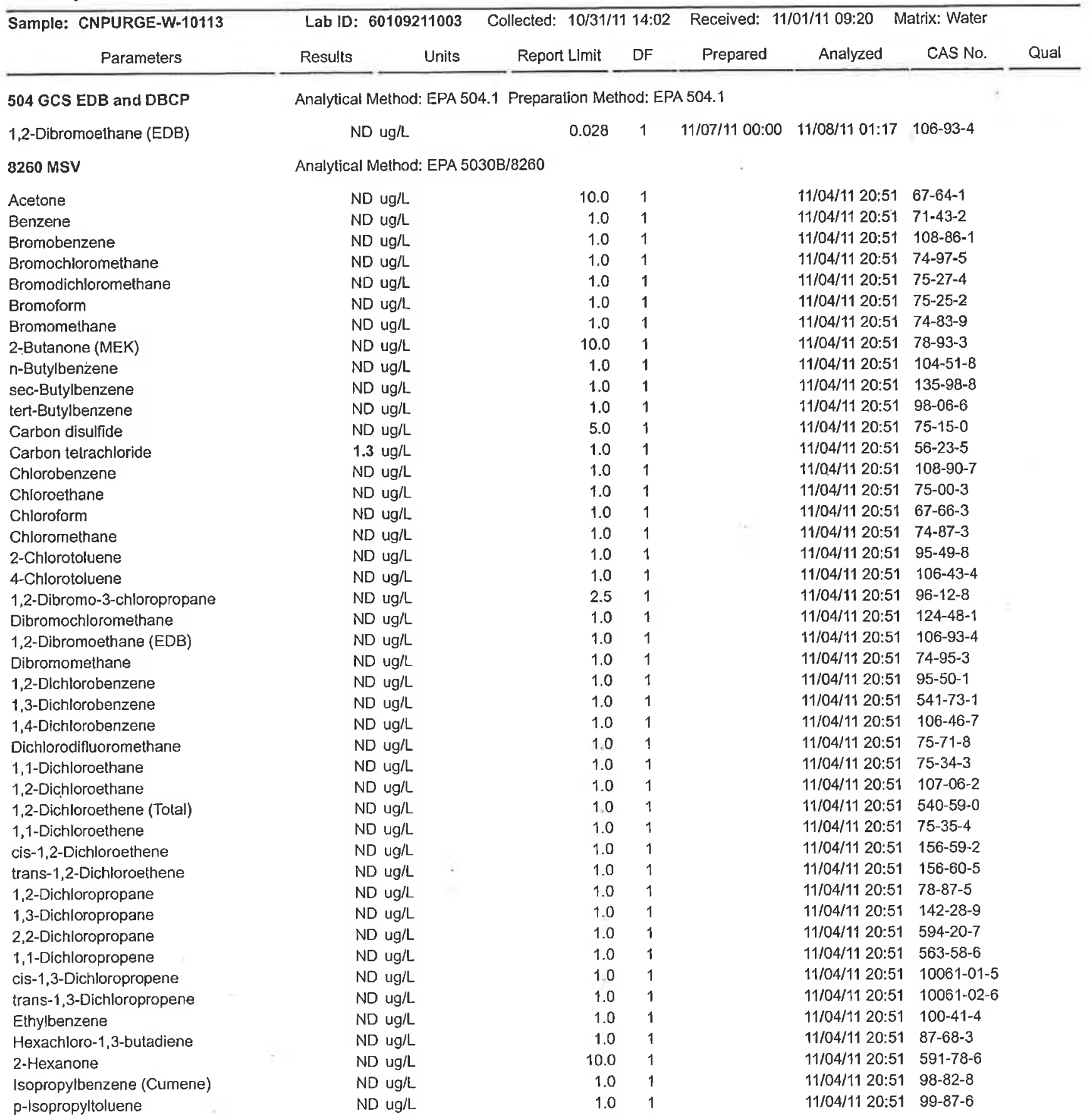

\section{REPORT OF LABORATORY ANALYSIS}




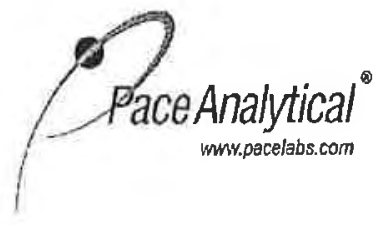

Pace Analytical Services, Inc. 9608 Loiret Blvd. Lenexa, KS 66219

(913)599-5665

ANALYTICAL RESULTS

Project: $\quad$ KS/MO Waste Water

Pace Project No.: $\quad 60109211$

Sample: CNPURGE-W-10113 Lab ID: 60109211003 Collected: 10/31/11 14:02 Received: 11/01/11 09:20 Matrix: Water

Parameters $\quad$ Results 1 Units Report Limit DF CAS No.

$8260 \mathrm{MSV}$

Methylene chloride

4-Methyl-2-pentanone (MIBK)

Methyl-ter-butyl ether

Naphthalene

n-Propylbenzene

Styrene

1,1,1,2-Tetrachloroethane

$1,1,2,2$-Tetrachloroethane

Tetrachioroethene

Toluene

1,2,3-Trichlorobenzene

1,2,4-Trichlorobenzene

$1,1,1$-Trichloroethane

1,1,2-Trichloroethane

Trichloroethene

Trichlorofluoromethane

1,2,3-Trichloropropane

1,2,4-Trimethylbenzene

1,3,5-Trimethylbenzene

Vinyl chloride

Xylene (Total)

4-Bromofluorobenzene (S)

Dibromofluoromethane (S)

1,2-Dichloroethane-d4 (S)

Toluene-d8 (S)

Preservation $\mathrm{pH}$

353.2 Nitrogen, NO2/NO3 unpres

Nitrogen, Nitrate
Analytical Method: EPA 5030B/8260

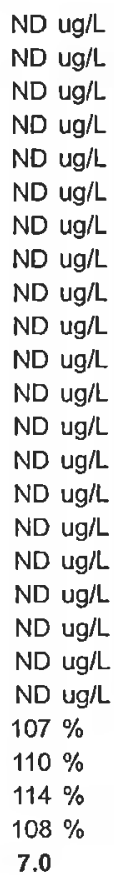

Analytical Method: EPA 353.2

$4.4 \mathrm{mg} / \mathrm{L}$

$\begin{array}{rr}1.0 & 1 \\ 10.0 & 1 \\ 1.0 & 1 \\ 10.0 & 1 \\ 1.0 & 1 \\ 1.0 & 1 \\ 1.0 & 1 \\ 1.0 & 1 \\ 1.0 & 1 \\ 1.0 & 1 \\ 1.0 & 1 \\ 1.0 & 1 \\ 1.0 & 1 \\ 1.0 & 1 \\ 1.0 & 1 \\ 1.0 & 1 \\ 2.5 & 1 \\ 1.0 & 1 \\ 1.0 & 1 \\ 1.0 & 1 \\ 3.0 & 1 \\ 87-113 & 1 \\ 86-112 & 1 \\ 82-119 & 1 \\ 90-110 & 1 \\ 0.10 & 1\end{array}$

0.101
11/04/11 20:51 75-09-2

11/04/11 20:51 108-10-1

$11 / 04 / 1120: 51 \quad 1634-04-4$

11/04/11 20:51 91-20-3

$11 / 04 / 1120: 51 \quad 103-65-1$

$11 / 04 / 1120: 51 \quad 100-42-5$

$11 / 04 / 1120: 51 \quad 630-20-6$

$11 / 04 / 1120: 51 \quad 79-34-5$

11/04/11 20:51 127-18-4

11/04/11 20:51 108-88-3

11/04/11 20:51 87-61-6

$11 / 04 / 1120: 51 \quad 120-82-1$

11/04/11 20:51 71-55-6

$11 / 04 / 1120: 51 \quad 79-00-5$

11/04/11 20:51 79-01-6

$11 / 04 / 1120: 51 \quad 75-69-4$

$11 / 04 / 1120: 51 \quad 96-18-4$

11/04/11 20:51 95-63-6

11/04/11 20:51 108-67-8

$11 / 04 / 1120: 51 \quad 75-01-4$

$\uparrow 1 / 04 / 11$ 20:51 1330-20-7

$11 / 04 / 1120: 51 \quad 460-00-4$

$11 / 04 / 1120: 51 \quad 1868-53-7$

$11 / 04 / 1120: 51 \quad 17060-07-0$

11/04/11 20:51 2037-26-5

11/04/11 20:51

$11 / 02 / 1109: 06$ 


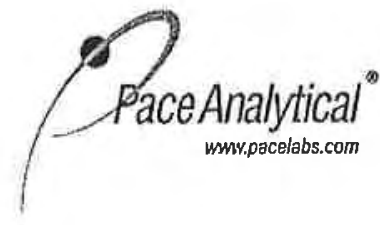

Pace Analytical Services, Inc. 9608 Loiret Blvd. Lenexa, KS 66219

(913)599-5665

\section{ANALYTICAL RESULTS}

Project: $\quad$ KS/MO Waste Water

Pace Project No.: 60109211

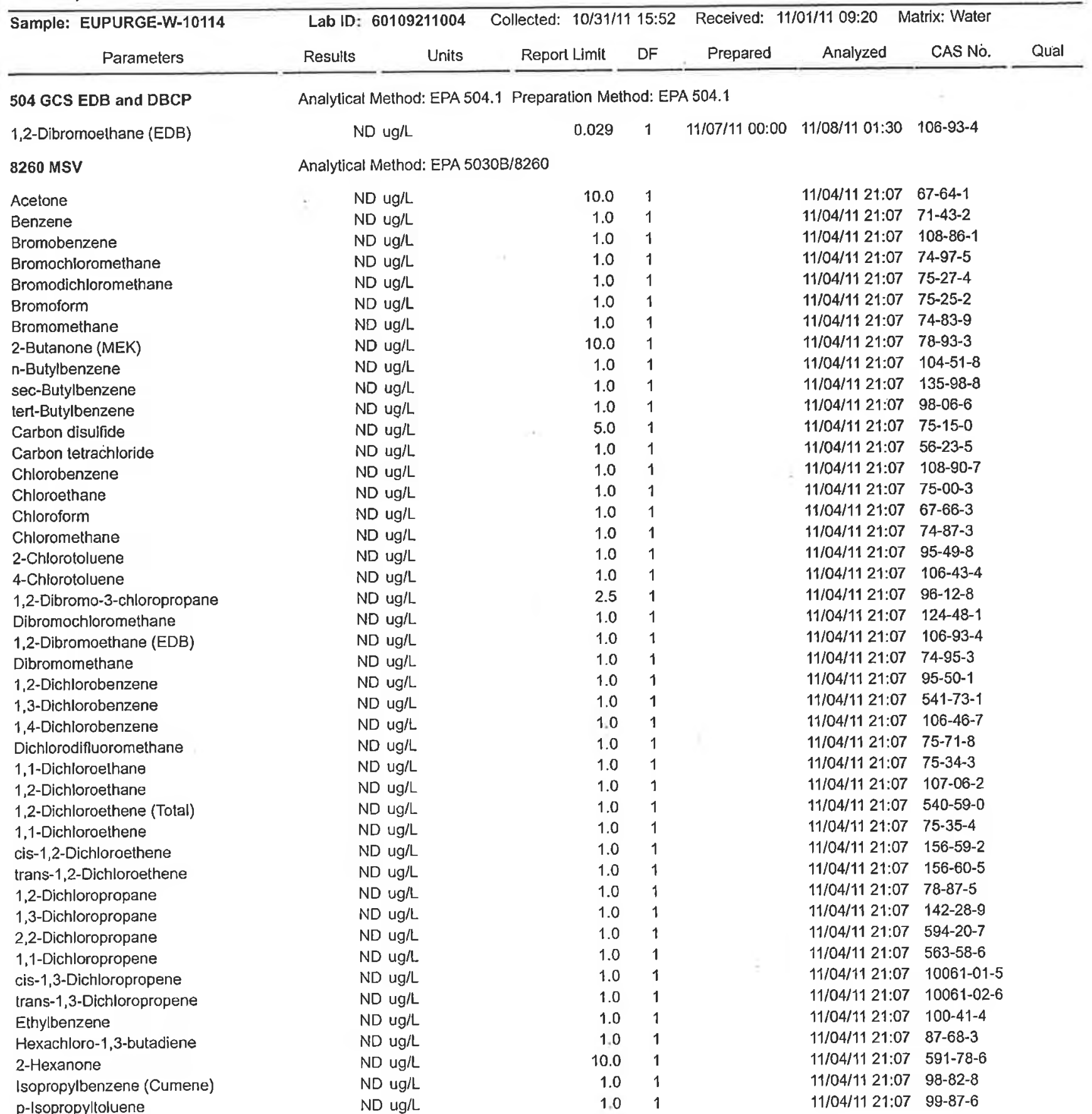

REPORT OF LABORATORY ANALYSIS Page 11 of 26

Date: 11/11/2011 11:15 AM

This report shall not be reproduced, except in full, without the written consent of Pace Analytical Services, Inc.. 
Pace Analytical Services, Inc. 9608 Loiret Blvd. Lenexa, KS 66219

\section{ANALYTICAL RESULTS}

Project: $\quad$ KS/MO Waste Water

Pace Project No.: 60109211

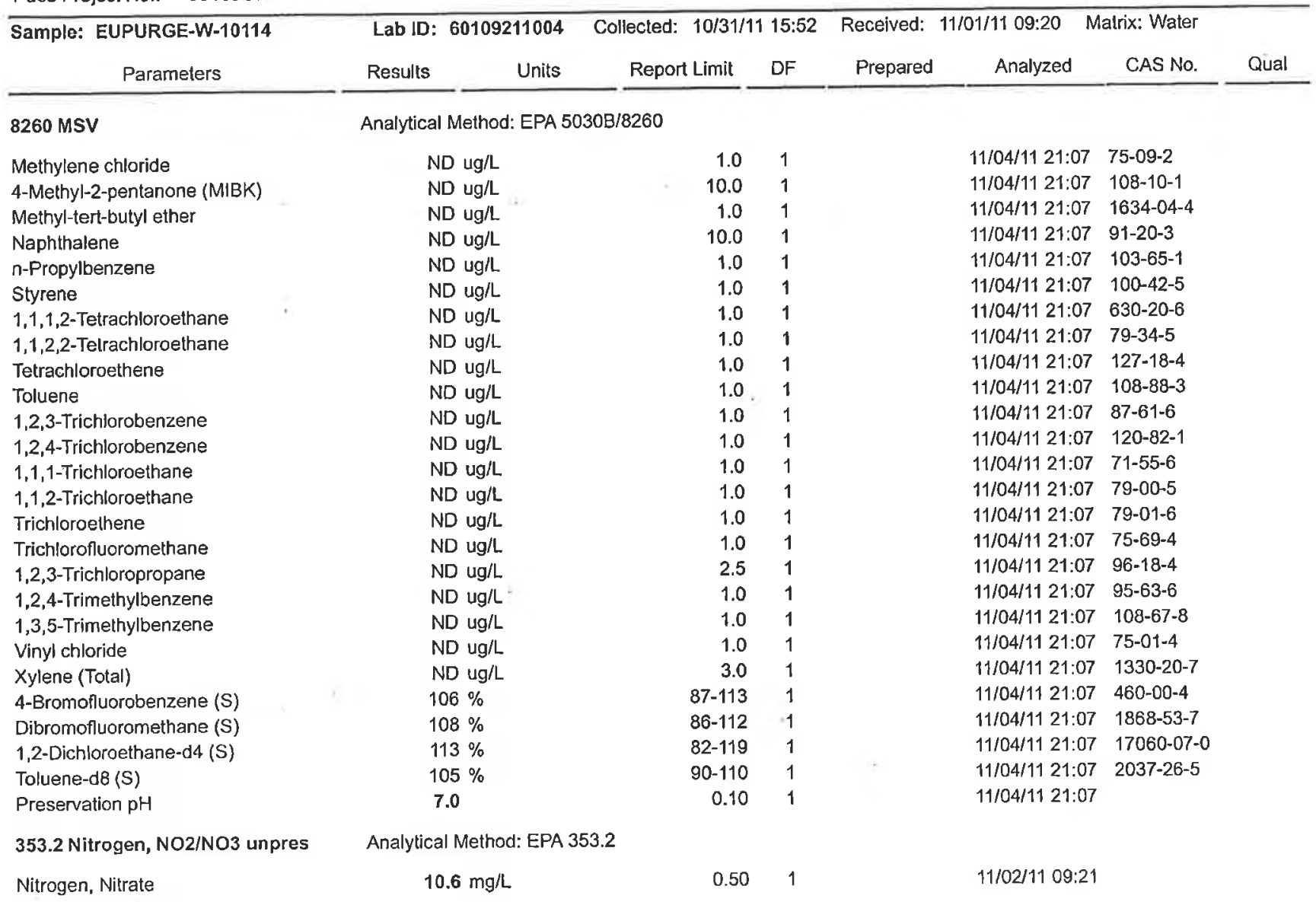




\section{ANALYTICAL RESULTS}

Froject: $\quad$ KS/MO Waste Water

Pace Project No: $\quad 60109211$

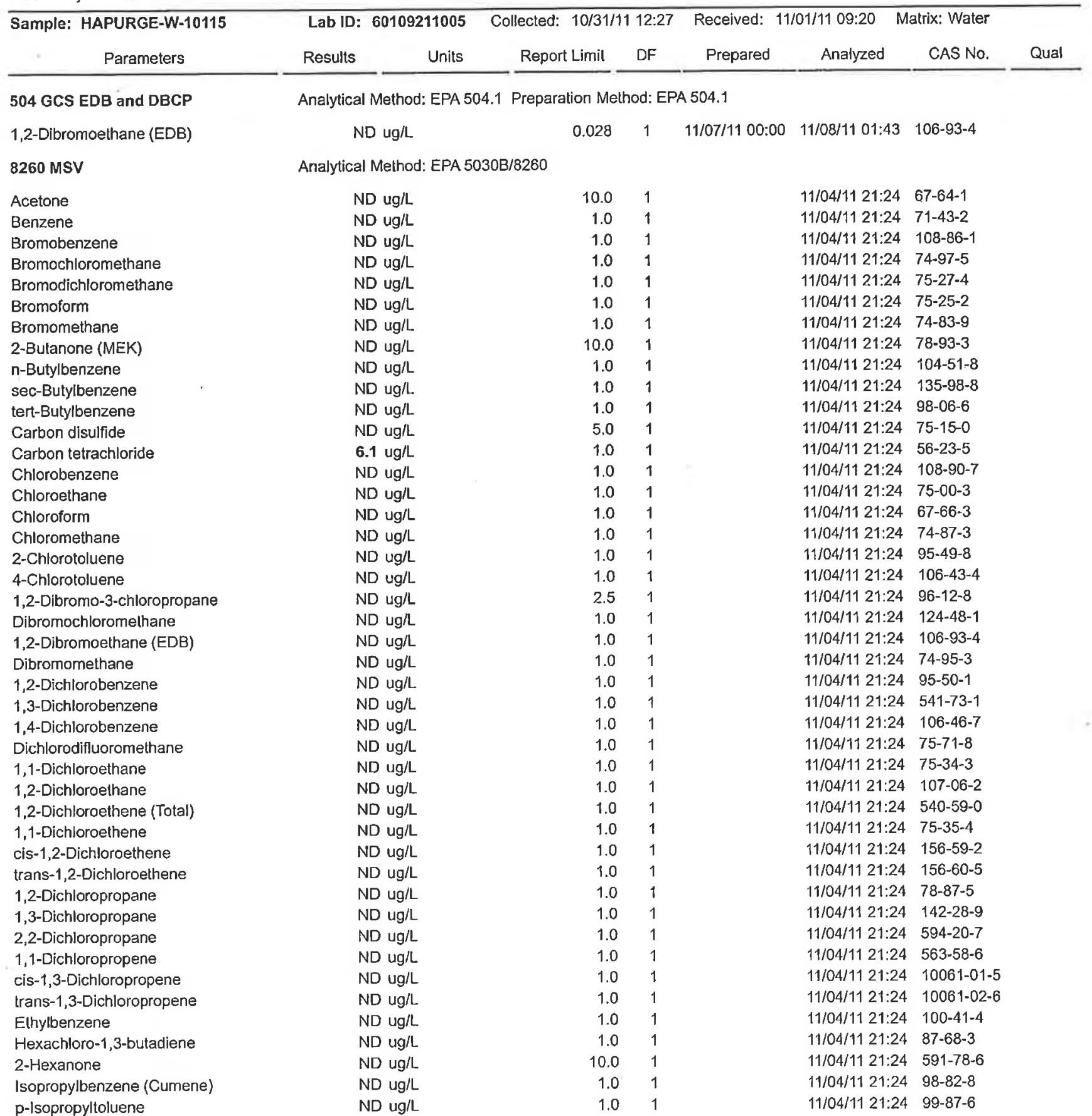

REPORT OF LABORATORY ANALYSIS Page 13 of 26

Date: $11 / 11 / 2011$ 11:15 AM

This report shall not be reproduced, except in full, without the written consent of Pace Analytical Services, Inc.. 


\section{ANALYTICAL RESULTS}

Project: $\quad$ KS/MO Waste Water

Pace Project No.: $\quad 60109211$

\begin{tabular}{|c|c|c|c|c|c|c|c|c|}
\hline \multirow{2}{*}{$\begin{array}{c}\text { Sample: HAPURGE-W-10115 } \\
\text { Parameters } \\
\end{array}$} & \multicolumn{2}{|c|}{ Lab ID: 60109211005} & Collected: & $10 / 31 / 1112: 27$ & \multirow{2}{*}{$\begin{array}{l}\text { Received: } \\
\text { Prepared }\end{array}$} & 11/01/11 09:20 & \multicolumn{2}{|l|}{ Matrix: Water } \\
\hline & Results & Units & Report Limit & DF & & . Analyzed & CAS No. & Qual \\
\hline 8260 MSV & Analytical & d: EPA 5 & $30 \mathrm{~B} / 8260$ & & & & & \\
\hline Methylene chloride & & & 1.0 & 1 & & $11 / 04 / 1121: 24$ & $75-09-2$ & \\
\hline 4-Methyl-2-pentanone (MIBK) & & & 10.0 & 1 & & $11 / 04 / 1121: 24$ & $108-10-1$ & \\
\hline Methyl-tert-butyl ether & & & 1.0 & 1 & & $11 / 04 / 1121: 24$ & $1634-04-4$ & \\
\hline Naphthalene & & & 10.0 & 1 & & $11 / 04 / 1121: 24$ & $91-20-3$ & \\
\hline n-Propylbenzene & & & 1.0 & 1 & & $11 / 04 / 1121: 24$ & $103-65-1$ & \\
\hline Styrene & & & 1.0 & 1 & & $11 / 04 / 1121: 24$ & $100-42-5$ & \\
\hline 1,1,1,2-Tetrachloroethane & & & 1.0 & 1 & & $11 / 04 / 1121: 24$ & $630-20-6$ & \\
\hline 1,1,2,2-Tetrachloroethane & & & 1.0 & 1 & & $11 / 04 / 1121: 24$ & $79-34-5$ & \\
\hline Tetrachloroethene & & & 1.0 & 1 & & $11 / 04 / 1121: 24$ & $127-18-4$ & \\
\hline Toluene & & & 1.0 & 1 & & $11 / 04 / 1121: 24$ & $108-88-3$ & \\
\hline 1,2,3-Trichlorobenzene & & & 1.0 & 1 & & $11 / 04 / 1121: 24$ & $87-61-6$ & \\
\hline 1,2,4-Trichlorobenzene & & & 1.0 & 1 & & $11 / 04 / 1121: 24$ & $120-82-1$ & \\
\hline 1,1,1-Trichloroethane & & & 1.0 & 1 & & $11 / 04 / 1121: 24$ & $71-55-6$ & \\
\hline 1,1,2-Trichloroethane & & & 1.0 & 1 & & $11 / 04 / 1121: 24$ & $79-00-5$ & \\
\hline Trichloroethene & & & 1.0 & 1 & & $11 / 04 / 1121: 24$ & $79-01-6$ & \\
\hline Trichlorofluoromethane & & & 1.0 & 1 & & $11 / 04 / 1121: 24$ & $75-69-4$ & \\
\hline 1,2,3-Trichloroprópane & & & 2.5 & 1 & & $11 / 04 / 1121: 24$ & $96-18-4$ & \\
\hline $1,2,4$-Trimethylbenzene & & & 1.0 & 1 & & $11 / 04 / 1121: 24$ & $95-63-6$ & \\
\hline 1,3,5-Trimethylbenzene & & & 1.0 & 1 & & $11 / 04 / 1121: 24$ & $108-67-8$ & \\
\hline Vinyl chloride & & & 1.0 & 1 & & $11 / 04 / 1121: 24$ & $75-01-4$ & \\
\hline Xylene (Total) & & & 3.0 & 1 & & $11 / 04 / 1121: 24$ & $1330-20-7$ & \\
\hline 4-Bromofluorobenzene (S) & & & $87-113$ & 1 & & $11 / 04 / 1121: 24$ & $460-00-4$ & \\
\hline Dibromofluoromethane (S) & & & $86-112$ & 1 & & $11 / 04 / 1121: 24$ & $1868-53-7$ & \\
\hline 1,2-Dichloroethane-d4 (S) & & & $82-119$ & 1 & & $11 / 04 / 1121: 24$ & $17060-07-0$ & \\
\hline Toluene-d8 (S) & & & $90-110$ & 1 & & $11 / 04 / 1121: 24$ & $2037-26-5$ & \\
\hline Preservation pH & 7 & & 0.10 & 1 & & $11 / 04 / 1121: 24$ & & \\
\hline 353.2 Nitrogen, $\mathrm{NO} / \mathrm{NO} 3$ unpres & Analytic & : EPA 3 & 3.2 & & & & & \\
\hline Nitrogen, Nitrate & & & 0.20 & 1 & & 11/02/11 09:16 & & \\
\hline
\end{tabular}




\section{ANALYTICAL RESULTS}

Project: $\quad$ KS/MO Waste Water

Pace Project No: $\quad 60109211$

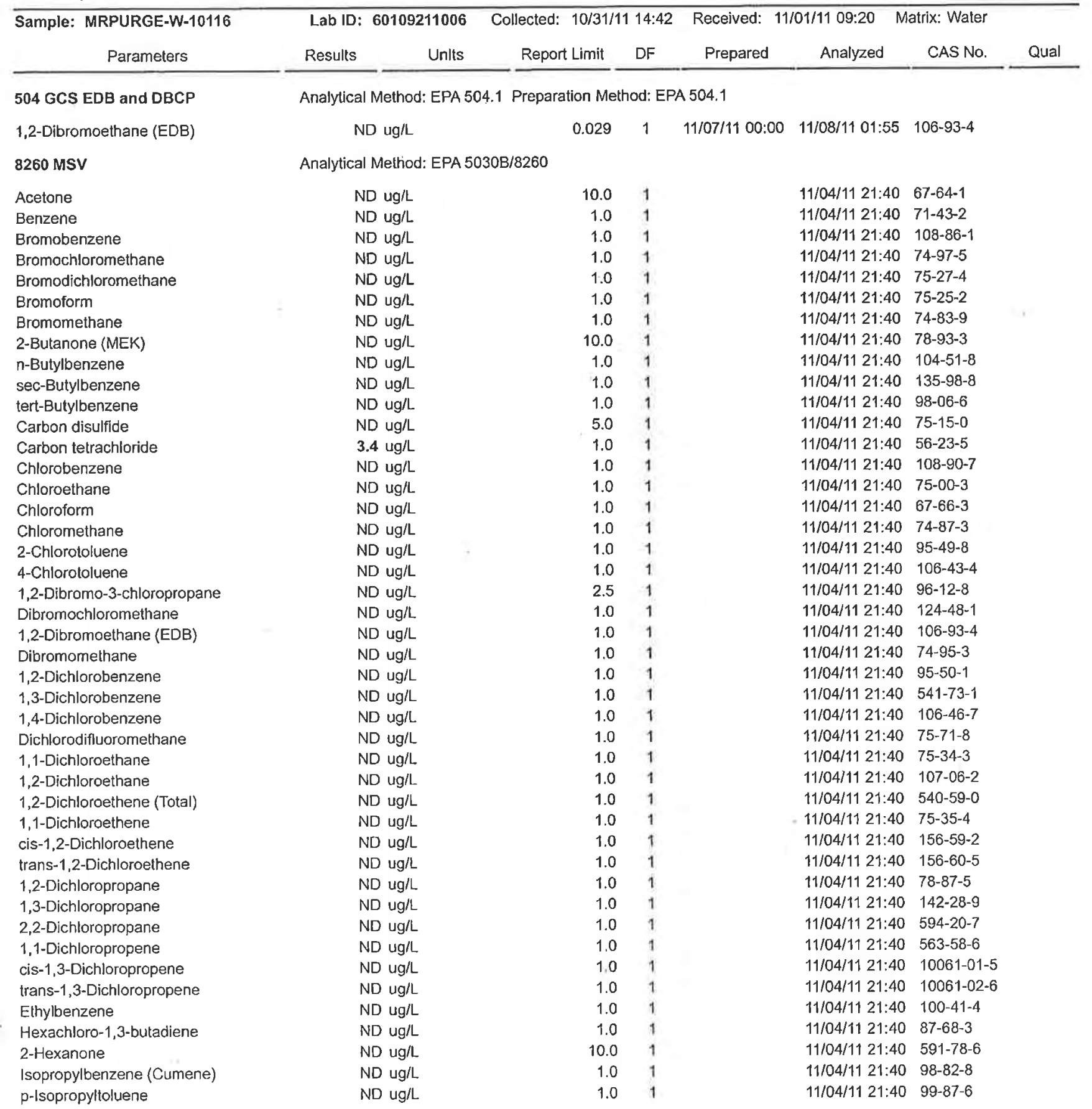

Date: 11/11/2011 11:15 AM

\section{REPORT OF LABORATORY ANALYSIS}




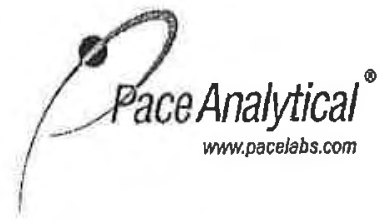

Pace Analytical Services, Inc. 9608 Loiret Blvd. Lenexa, KS 66219

(913)599-5665

ANALYTICAL RESULTS

Project: $\quad$ KS/MO Waste Water

Pace Project No.: 60109211

\begin{tabular}{|c|c|c|c|c|c|c|c|c|c|}
\hline $\begin{array}{c}\text { Sample: MRPURGE-W-10116 } \\
\text { Parameters }\end{array}$ & $\begin{array}{l}\text { Lab ID: } 6 \\
\text { Results }\end{array}$ & $\begin{array}{r}60109211006 \\
\text { Units }\end{array}$ & $\begin{array}{r}\text { Collected: } \\
\text { Report }\end{array}$ & $\begin{array}{l}10 / 31 / 1 \\
t \text { Limit }\end{array}$ & $\begin{array}{l}14: 42 \\
\text { DF }\end{array}$ & $\begin{array}{l}\text { Received: } 1 \\
\text { Prepared }\end{array}$ & $\begin{array}{c}11 / 01 / 1109: 20 \quad M \\
\text { Analyzed } \\
\end{array}$ & $\begin{array}{l}\text { atrix: Water } \\
\text { CAS No. }\end{array}$ & Qual \\
\hline 8260 MSV & Analytical N & Method: EPA 50 & $30 \mathrm{~B} / 8260$ & & & & & & \\
\hline Methylene chloride & ND & $\mathrm{ug} / \mathrm{L}$ & & 1.0 & 1 & & $11 / 04 / 1121: 40$ & $75-09-2$ & \\
\hline 4-Methyl-2-pentanone (MIBK) & ND & $u g / L$ & & 10.0 & 1 & & $11 / 04 / 1121: 40$ & $108-10-1$ & \\
\hline Methyl-tert-bulyl ether & ND & $u g / L$ & & 1.0 & 1 & & $11 / 04 / 1121: 40$ & $1634-04-4$ & \\
\hline Naphthalene & ND & $\mathrm{ug} / \mathrm{L}$ & & 10.0 & 1 & & $11 / 04 / 1121: 40$ & $91-20-3$ & \\
\hline n-Propylbenzene & ND & $\mathrm{ug} / \mathrm{L}$ & & 1.0 & 1 & & 11/04/11 21:40 & $103-65-1$ & \\
\hline Styrene & ND & $\mathrm{ug} / \mathrm{L}$ & & 1.0 & 1 & & $11 / 04 / 1121: 40$ & $100-42-5$ & \\
\hline $1,1,1,2$-Tetrachloroethane & ND & $\mathrm{ug} / \mathrm{L}$ & & 1.0 & 1 & & $11 / 04 / 1121: 40$ & $630-20-6$ & \\
\hline $1,1,2,2$-Tetrachloroethane & ND & $\mathrm{ug} / \mathrm{L}$ & & 1.0 & 1 & & $11 / 04 / 1121: 40$ & $79-34-5$ & \\
\hline Tetrachloroethene & ND & $u g / L$ & & 1.0 & 1 & & $11 / 04 / 1121: 40$ & $127-18-4$ & \\
\hline Toluene & ND & $\mathrm{ug} / \mathrm{L}$ & & 1.0 & 1 & & $11 / 04 / 1121: 40$ & $108-88-3$ & \\
\hline 1,2,3-Trichlorobenzene & ND & $\mathrm{ug} / \mathrm{L}$ & & 1.0 & 1 & & $11 / 04 / 1121: 40$ & $87-61-6$ & \\
\hline 1,2,4-Trichlorobenzene & ND & $\mathrm{ug} / \mathrm{L}$ & & 1.0 & 1 & & $11 / 04 / 1121: 40$ & $120-82-1$ & \\
\hline 1,1,1-Trichloroelhane & ND & ug/L & & 1.0 & 1 & & $11 / 04 / 1121: 40$ & $71-55-6$ & \\
\hline 1,1,2-Trichloroethane & ND & ug/L & & 1.0 & 1 & & $11 / 04 / 1121: 40$ & $79-00-5$ & \\
\hline Trichloroethene & ND & ug/L & & 1.0 & 1 & & $11 / 04 / 1121: 40$ & $79-01-6$ & \\
\hline Trichlorofluoromethane & ND & ug/L & & 1.0 & 1 & & $11 / 04 / 1121: 40$ & $75-69-4$ & \\
\hline 1,2,3-Trichloropropane & ND & ug/L & & 2.5 & 1 & & $11 / 04 / 1121: 40$ & $96-18-4$ & \\
\hline 1,2,4-Trimethylbenzene & ND & ug/L & & 1.0 & 1 & & $11 / 04 / 1121: 40$ & $95-63-6$ & \\
\hline 1,3,5-Trimethylbenzene & ND & $\mathrm{ug} / \mathrm{L}$ & & 1.0 & 1 & & $11 / 04 / 1121: 40$ & $108-67-8$ & \\
\hline Vinyl chloride & ND & $\mathrm{ug} / \mathrm{L}$ & & 1.0 & 1 & & $11 / 04 / 1121: 40$ & $75-01-4$ & \\
\hline Xylene (Total) & ND & $\mathrm{ug} / \mathrm{L}$ & & 3.0 & 1 & & $11 / 04 / 1121: 40$ & $1330-20-7$ & \\
\hline 4-Bromofluorobenzene (S) & 105 & & & $87-113$ & 1 & & $11 / 04 / 1121: 40$ & $460-00-4$ & \\
\hline Dibromofluoromethane (S) & 108 & & & $86-112$ & 1 & & $11 / 04 / 1121: 40$ & $1868-53-7$ & \\
\hline 1,2-Dichloroethane-d4 (S) & 114 & $4 \%$ & & $82-119$ & 1 & . & $11 / 04 / 1121: 40$ & $17060-07-0$ & \\
\hline Toluene-d8 (S) & 109 & & & $90-110$ & 1 & & $11 / 04 / 1121: 40$ & $2037-26-5$ & \\
\hline Preservation $\mathrm{pH}$ & 7.0 & & & 0.10 & 1 & & $11 / 04 / 1121: 40$ & $\rightarrow$ & \\
\hline 353.2 Nitrogen, NO2/NO3 unpres & Analytical $\wedge$ & Method: EPA 3 & 3.2 & & & & & & \\
\hline Nitrogen, Nitrate & 13.8 & $3 \mathrm{mg} / \mathrm{L}$ & & 0.50 & 1 & & $11 / 02 / 1109: 20$ & & \\
\hline
\end{tabular}




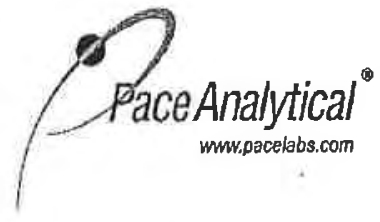

Pace Analytical Services, Inc. 9608 Loirel Bivd. Lenexa, KS 66219

(913)599-5665

ANALYTICAL RESULTS

Project: $\quad$ KS/MO Waste Water

Pace Project No.: 60109211

. Sample: SVPURGE-W-10117 Lab ID: 60109211007 Collected: 10/31/11 18:30 Received: 11/01/11 09:20 Matrix: Water

$\frac{\text { Parameters }}{504 \text { GCS EDB and DBCP }} \frac{\text { Results }}{\text { Analytical Method: EPA 504.1 Preparation Method: EPA 504.1 }} \frac{\text { Units }}{\text { Report Limit }} \stackrel{\text { DF }}{\text { Prepared }}$

504 GCS EDB and DBCP

$\begin{array}{lllllll}\text { ND ug/L } \quad 0.029 & 1 & 11 / 07 / 11 & 00: 00 & 11 / 08 / 1102: 09 & 106-93-4\end{array}$

1,2-Dibromoethane (EDB)

$8260 \mathrm{MSV}$

Acetone

Benzene

Bromobenzene

Bromochloromethane

Bromodichloromethane

Bromoform

Bromomethane

2-Butanone (MEK)

n-Butylbenzene

sec-Butylbenzene

tert-Butylbenzene

Carbon disulfide

Carbon tetrachloride

Chlorobenzene

Chloroethane

Chloroform

Chloromethane

2-Chlorotoluene

4-Chlorotoluene

1,2-Dibromo-3-chloropropane

Dibromochloromethane

1,2-Dibromoethane (EDB)

Dibromomethane

1,2-Dichlorobenzene

1,3-Dichlorobenzene

1,4-Dichlorobenzene

Dichlorodifluoromethane

1,1-Dichloroethane

1,2-Dichloroethane

1,2-Dichloroethene (Total)

1,1-Dichloroethene

cis-1,2-Dichloroethene

trans-1,2-Dichloroethene

1,2-Dichloropropane

1,3-Dichloropropane

2,2-Dichloropropane

1,1-Dichloropropene

cis-1,3-Dichloropropene

trans-1,3-Dichloropropene

Ethylbenzene

Hexachloro-1,3-butadiene

2-Hexanone

Isopropylbenzene (Cumene)

p-Isopropyltoluene

Date: 11/11/2011 11:15 AM
Analytical Method: EPA 5030B/8260

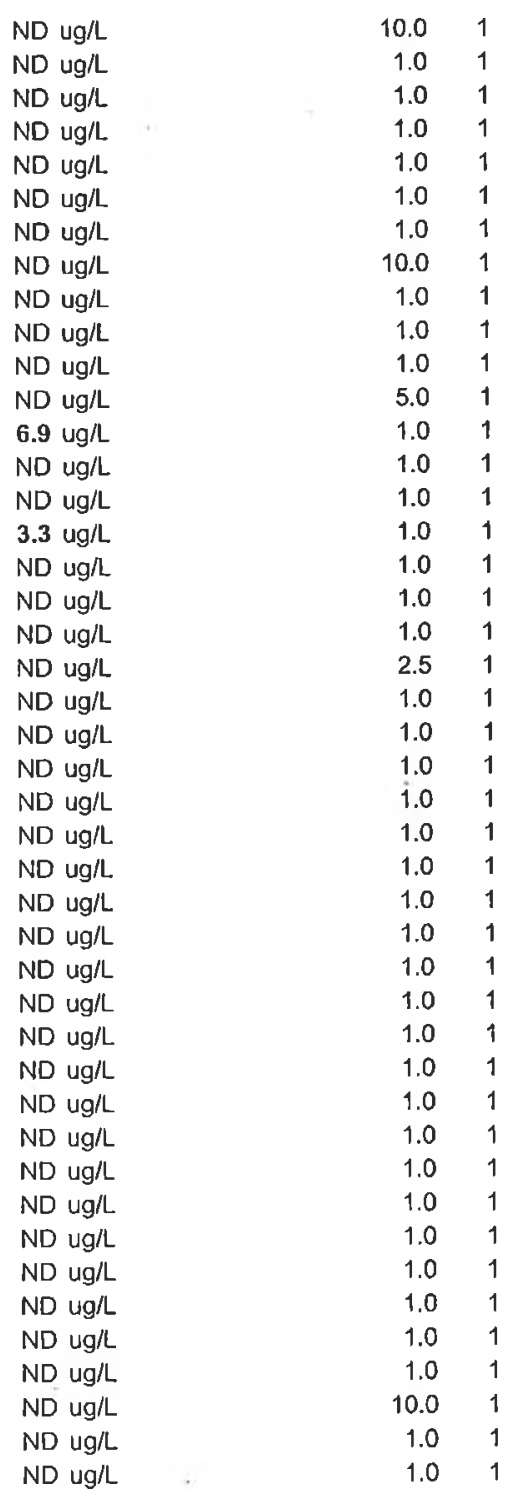

11/04/11 21:56 67-64-1

11/04/11 21:56 71-43-2

11/04/11 21:56 108-86-1

11/04/11 21:56 74-97-5

$11 / 04 / 1121: 56 \quad 75-27-4$

11/04/11 21:56 75-25-2

11/04/11 21:56 74-83-9

$11 / 04 / 1121: 56 \quad 78-93-3$

$11 / 04 / 1121: 56 \quad 104-51-8$

$11 / 04 / 1121: 56 \quad 135-98-8$

11/04/11 21:56 98-06-6

11/04/11 21:56 75-15-0

11/04/11 21:56 56-23-5

11/04/11 21:56 108-90-7

11/04/11 21:56 75-00-3

$11 / 04 / 1121: 56 \quad 67-66-3$

$11 / 04 / 1121: 56 \quad 74-87-3$

$11 / 04 / 1121: 56 \quad 95-49-8$

$11 / 04 / 1121: 56 \quad 106-43-4$

$11 / 04 / 1121: 56 \quad 96-12-8$

$11 / 04 / 1121: 56 \quad 124-48-1$

11/04/11 21:56 106-93-4

11/04/11 21:56 74-95-3

11/04/11 21:56 95-50-1

11/04/11 21:56 541-73-1

11/04/11 21:56 106-46-7

$11 / 04 / 1121: 56 \quad 75-71-8$

11/04/11 21:56 75-34-3

11/04/11 21:56 107-06-2

11/04/11 21:56 540-59-0

11/04/11 21:56 75-35-4

11/04/11 21:56 156-59-2

$11 / 04 / 1121: 56 \quad 156-60-5$

11/04/11 21:56 78-87-5

$11 / 04 / 1121: 56 \quad 142-28-9$

11/04/11 21:56 594-20-7

11/04/11 21:56 563-58-6

11/04/11 21:56 10061-01-5

11/04/11 21:56 10061-02-6

11/04/11 21:56 100-41-4

$11 / 04 / 1121: 56 \quad 87-68-3$

$11 / 04 / 1121: 56 \quad 591-78-6$

11/04/11 21:56 98-82-8

11/04/11 21:56 99-87-6

\section{REPORT OF LABORATORY ANALYSIS}


Pace Analytical Services, Inc. 9608 Loiret Blvd. Lenexa, KS 66219

\section{ANALYTICAL RESULTS}

Project: $\quad$ KS/MO Waste Water

Pace Project No.: 60109211

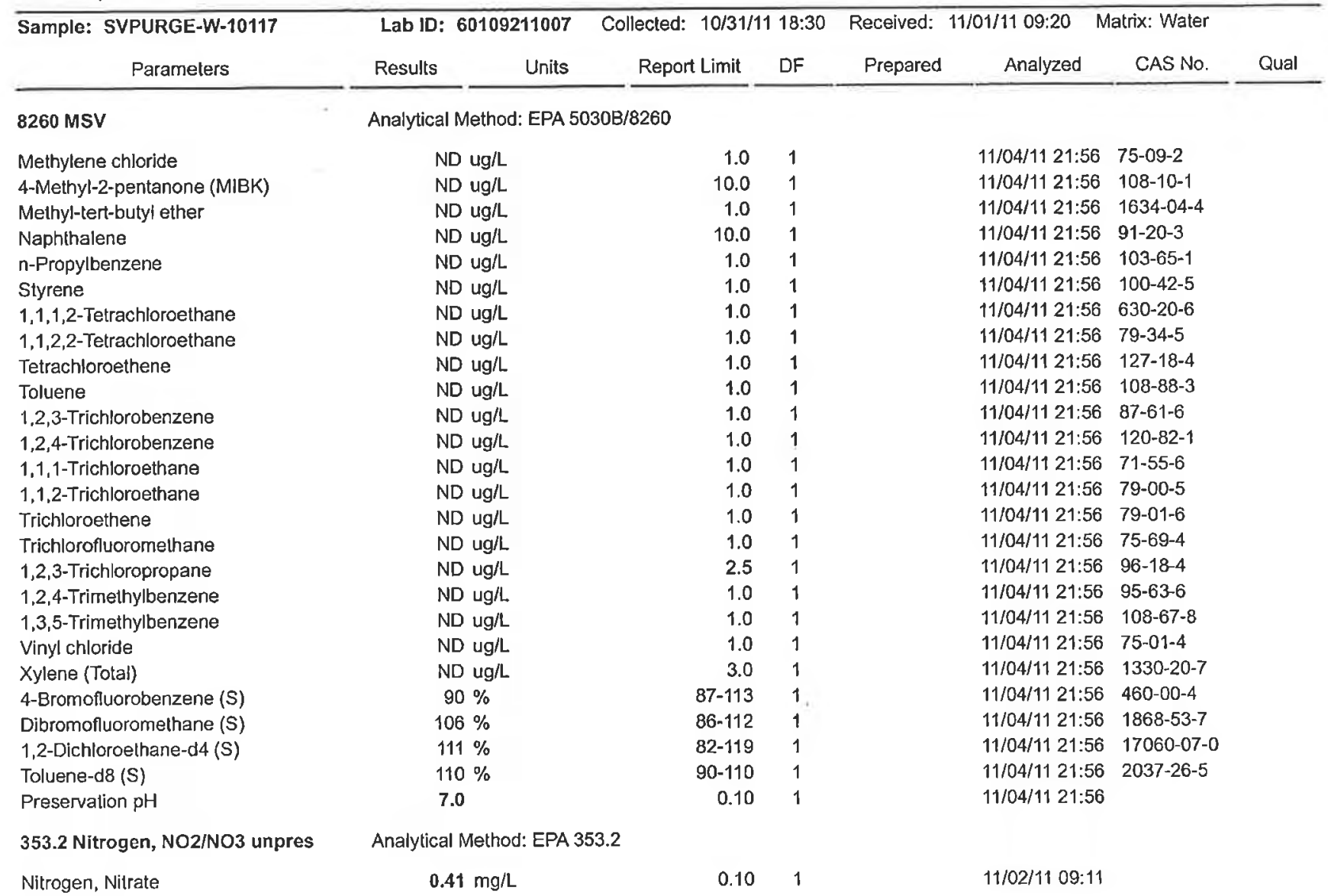




\section{QUALITY CONTROL DATA}

Project: $\quad$ KS/MO Wasle Water

Pace Project No.: 60109211

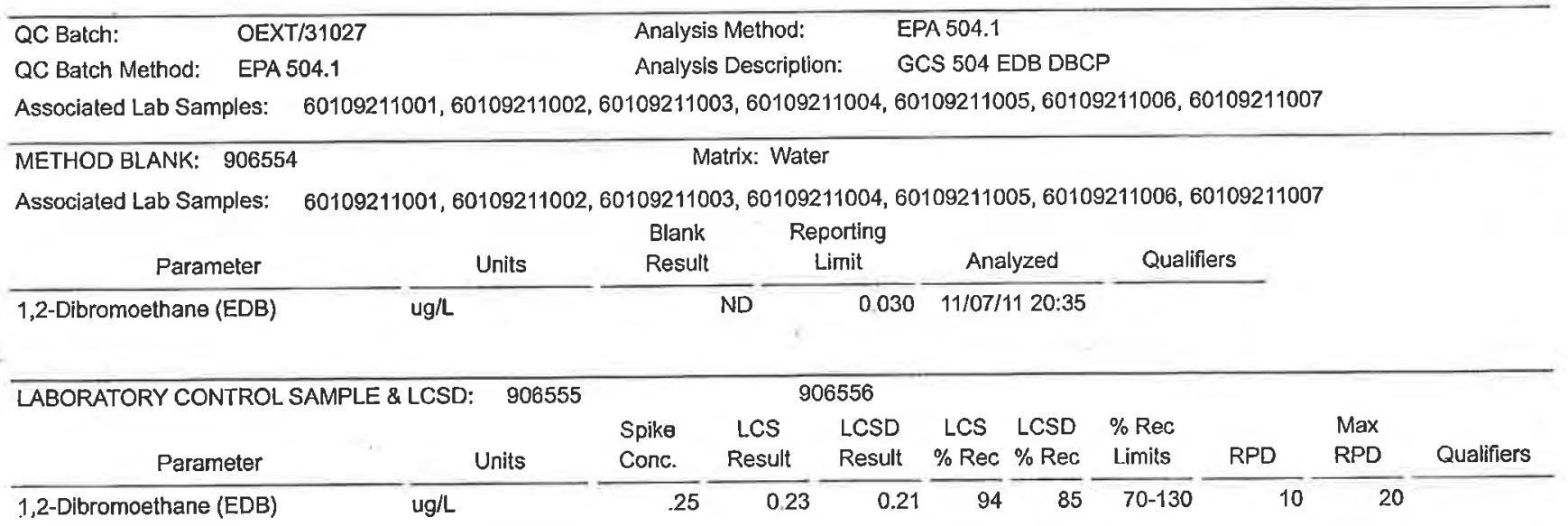




\section{QUALITY CONTROL DATA}

Project: $\quad$ KS/MO Waste Water

Pace Project No.: $\quad 60109211$

\begin{tabular}{llll}
\hline QC Batch: & MSV/41422 & Analysis Method: & EPA 5030B/8260 \\
QC Batch Method: & EPA 5030B/8260 & Analysis Description: & 8260 MSV Water 7 day \\
Associated Lab Samples: & $60109211001,60109211002,60109211003,60109211004,60109211005,60109211006,60109211007$
\end{tabular}

METHOD BLANK: $905182 \quad$ Matrix: Water
$\begin{array}{ll}\text { Associated Lab Samples: } & 60109211001,60109211002,60109211003,60109211004,60109211005,60109211006,60109211007\end{array}$

Associated Lab Samples: $\quad 60109211001,60109211002,60109211003,60109211004,60109211005,60109211006,60109211007$

\begin{tabular}{|c|c|c|c|c|c|}
\hline Parameter & Units & $\begin{array}{l}\text { Blank } \\
\text { Result }\end{array}$ & $\begin{array}{c}\text { Reporting } \\
\text { Limit }\end{array}$ & Analyzed & Qualifiers \\
\hline $1,1,1,2-$-Tetrachloroethane & $\mathrm{ug} / \mathrm{L}$ & ND & 1.0 & $11 / 04 / 1117: 52$ & \\
\hline 1,1,1-Trichloroethane & $\mathrm{ug} / \mathrm{L}$ & ND & 1.0 & $11 / 04 / 1117: 52$ & \\
\hline 1,1,2,2-Tetrachloroethane & $\mathrm{ug} / \mathrm{L}$ & ND & 1.0 & $11 / 04 / 1117: 52$ & \\
\hline 1,1,2-Trichloroethane & $\mathrm{ug} / \mathrm{L}$ & ND & 1.0 & $11 / 04 / 1117: 52$ & \\
\hline 1,1-Dichloroethane & ug/L & ND & 1.0 & $11 / 04 / 1117: 52$ & \\
\hline 1,1-Dichloroethene & $\mathrm{ug} / \mathrm{L}$ & ND & 1.0 & $11 / 04 / 1117: 52$ & \\
\hline 1,1-Dichloropropene & $\mathrm{ug} / \mathrm{L}$ & ND & 1.0 & $11 / 04 / 1117: 52$ & \\
\hline 1,2,3-Trichlorobenzene & $\mathrm{ug} / \mathrm{L}$ & ND & 1.0 & $11 / 04 / 1117: 52$ & \\
\hline 1,2,3-Trichloropropane & $\mathrm{ug} / \mathrm{L}$ & ND & 2.5 & $11 / 04 / 1117: 52$ & \\
\hline 1,2,4-Trichlorobenzene & ug/L & ND & 1.0 & $11 / 04 / 1117: 52$ & \\
\hline 1,2,4-Trimethylbenzene & $u g / L$ & ND & 1.0 & $11 / 04 / 1117: 52$ & \\
\hline 1,2-Dibromo-3-chloropropane & $\mathrm{ug} / \mathrm{L}$ & ND & 2.5 & $11 / 04 / 1117: 52$ & \\
\hline 1,2-Dibromoethane (EDB) & $\mathrm{ug} / \mathrm{L}$ & ND & 1.0 & $11 / 04 / 1117: 52$ & \\
\hline 1.2-Dichlorobenzene & $\mathrm{ug} / \mathrm{L}$ & ND & 1.0 & $11 / 04 / 1117: 52$ & \\
\hline 1,2-Dichloroethane & $\mathrm{ug} / \mathrm{L}$ & ND & 1.0 & $11 / 04 / 1117: 52$ & \\
\hline 1,2-Dichloroethene (Total) & ug/L & ND & 1.0 & $11 / 04 / 1117: 52$ & \\
\hline 1,2-Dichloropropane & ug/L & ND & 1.0 & $11 / 04 / 1117: 52$ & \\
\hline 1,3,5-Trimethylbenzene & $\mathrm{ug} / \mathrm{L}$ & ND & 1.0 & $11 / 04 / 1117: 52$ & \\
\hline 1,3-Dichlorobenzene & $u g / L$ & ND & 1.0 & $11 / 04 / 1117: 52$ & \\
\hline 1,3-Dichloropropane & $\mathrm{ug} / \mathrm{L}$ & ND & 1.0 & $11 / 04 / 1117: 52$ & \\
\hline 1,4-Dichlorobenzene & $\mathrm{ug} / \mathrm{L}$ & ND & 1.0 & 11/04/11 17:52 & \\
\hline 2,2-Dichloropropane & $\mathrm{ug} / \mathrm{L}$ & ND & 1.0 & 11/04/11 17:52 & \\
\hline 2-Butanone (MEK) & $\mathrm{ug} / \mathrm{L}$ & ND & 10.0 & $11 / 04 / 1117: 52$ & \\
\hline 2-Chlorotoluene & $\mathrm{ug} / \mathrm{L}$ & ND & 1.0 & $11 / 04 / 1117: 52$ & \\
\hline 2-Hexanone & $u g / L$ & ND & 10.0 & $11 / 04 / 1117: 52$ & \\
\hline 4-Chlorololuene & $\mathrm{ug} / \mathrm{L}$ & ND & 1.0 & $11 / 04 / 1117: 52$ & \\
\hline 4-Melhyl-2-pentanone (MIBK) & $\mathrm{ug} / \mathrm{L}$ & ND & 10.0 & 11/04/11 17:52 & \\
\hline Acelone & $u g / L$ & ND & 10.0 & $11 / 04 / 1117: 52$ & \\
\hline Benzene & $\mathrm{ug} / \mathrm{L}$ & ND & 1.0 & $11 / 04 / 1117: 52$ & \\
\hline Bromobenzene & $\mathrm{ug} / \mathrm{L}$ & ND & 1.0 & $11 / 04 / 1117: 52$ & \\
\hline Bromochloromethane & $\mathrm{ug} / \mathrm{L}$ & ND & 1.0 & $11 / 04 / 1117: 52$ & \\
\hline Bromodichloromelhane & $\mathrm{ug} / \mathrm{L}$ & ND & 1.0 & $11 / 04 / 1117: 52$ & \\
\hline Bromoform & $\mathrm{ug} / \mathrm{L}$ & ND & 1.0 & $11 / 04 / 1117: 52$ & \\
\hline Bromomethane & $\mathrm{ug} / \mathrm{L}$ & ND & 1.0 & $11 / 04 / 1117: 52$ & \\
\hline Carbon disulfide & ug/L & ND & 5.0 & $11 / 04 / 1117: 52$ & \\
\hline Carbon tetrachloride & $\mathrm{ug} / \mathrm{L}$ & ND & 1.0 & $11 / 04 / 1117: 52$ & \\
\hline Chlorobenzene & $\mathrm{ug} / \mathrm{L}$ & ND & 1.0 & $11 / 04 / 1117: 52$ & \\
\hline Chloroethane & $\mathrm{ug} / \mathrm{L}$ & ND & 1.0 & $11 / 04 / 11$ 17:52 & \\
\hline Chloroform & $\mathrm{ug} / \mathrm{L}$ & ND & 1.0 & $11 / 04 / 1117: 52$ & \\
\hline Chloromethane & $u g / L$ & ND & 1.0 & $11 / 04 / 1117: 52$ & \\
\hline cis-1,2-Dichloroethene & $\mathrm{ug} / \mathrm{L}$ & ND & 1.0 & $11 / 04 / 1117: 52$ & \\
\hline cis-1,3-Dichloropropene & $\mathrm{ug} / \mathrm{L}$ & ND & 1.0 & $11 / 04 / 1117: 52$ & \\
\hline Dibromochloromelhane & ug/L & ND & 1.0 & $11 / 04 / 1117: 52$ & \\
\hline
\end{tabular}




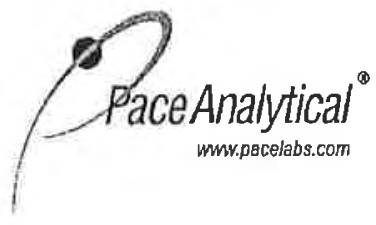

Pace Analytical Services, Inc. 9608 Loiret Blvd.

Lenexa, KS 66219

(913)599-5665

\section{QUALITY CONTROL DATA}

Project: $\quad$ KS/MO Waste Water

Pace Project No.: 60109211

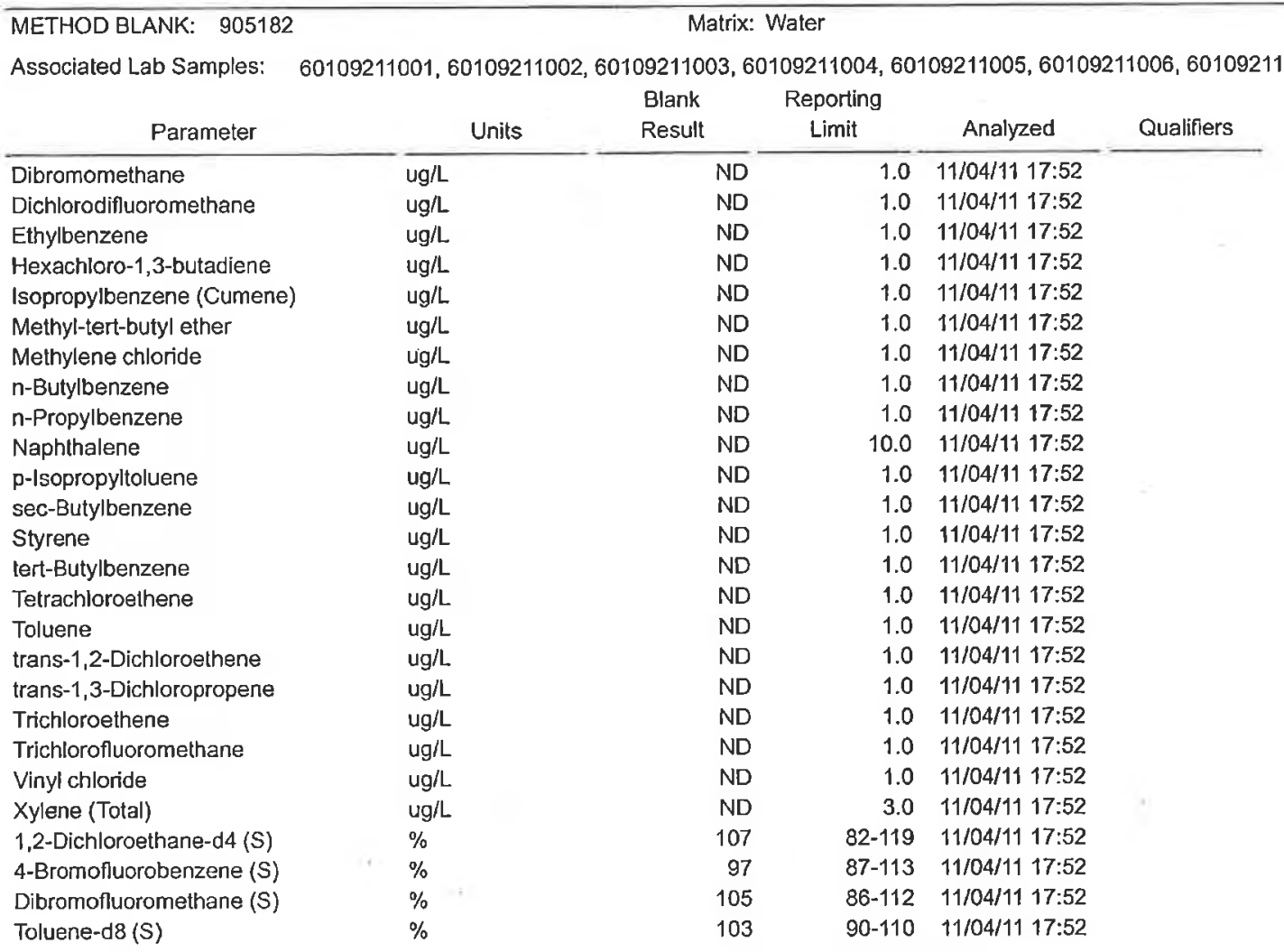

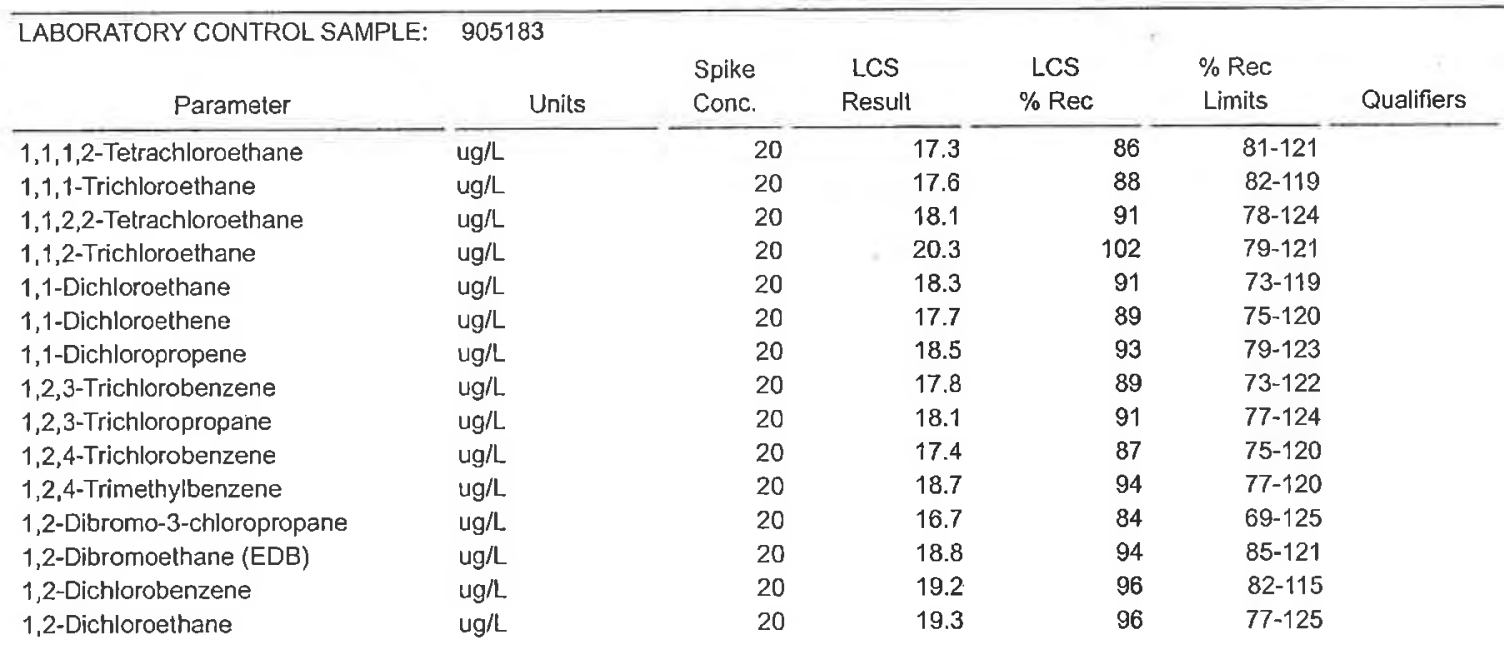




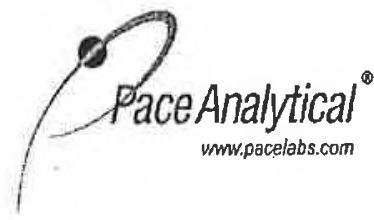

Pace Analytical Services, Inc. 9608 Loiret Blvd.

Lenexa, KS 66219

(913)599-5665

\section{QUALITY CONTROL DATA}

Project:

KS/MO Waste Water

Pace Project No.: $\quad 60109211$

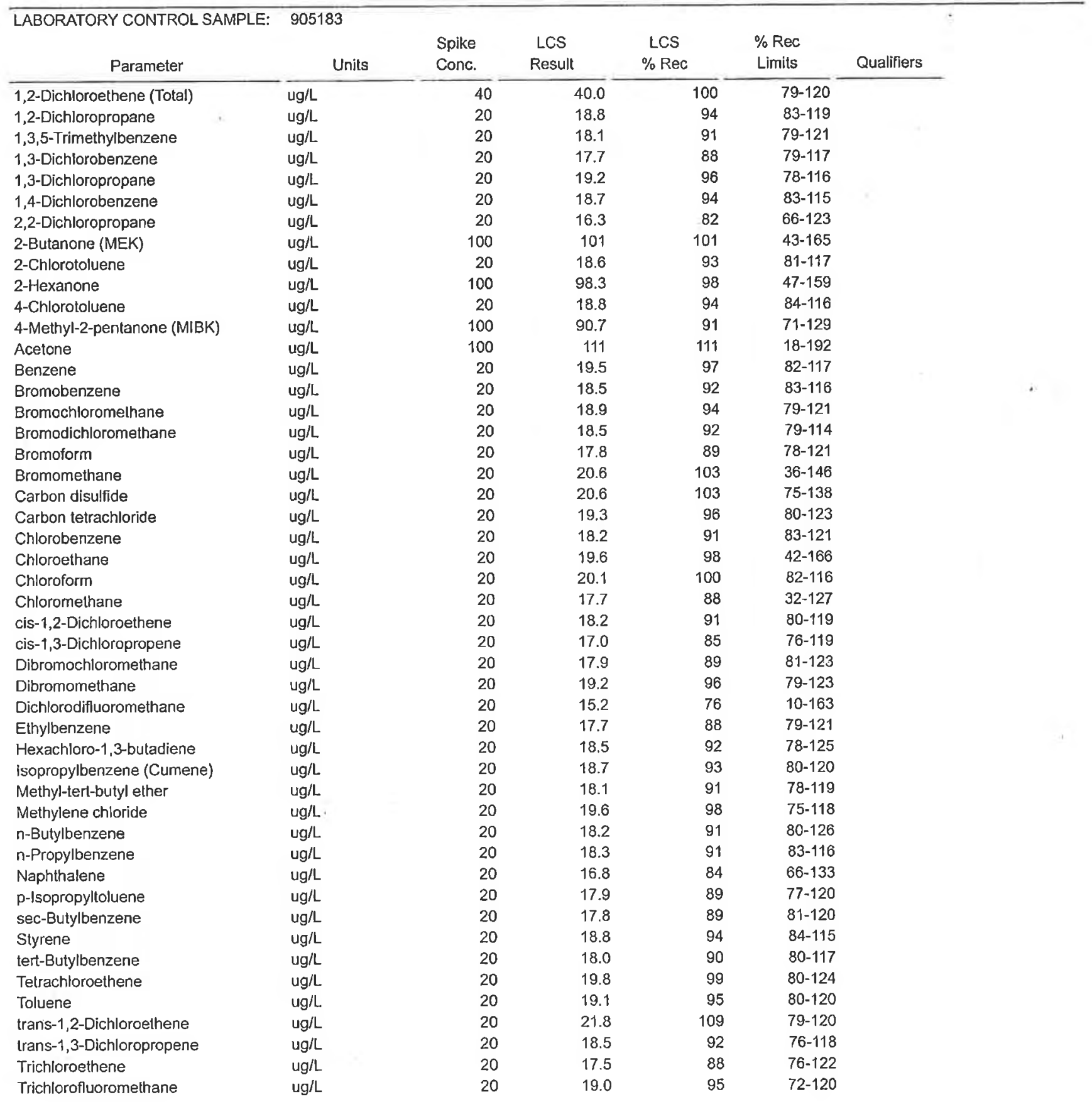

Date: $11 / 11 / 2011$ 11:15 AM 
Pace Analytlcal Services, Inc. 9608 Lolret Blyd.

Lenexa, KS 66219

(913)599-5665

\section{QUALITY CONTROL DATA}

Project: $\quad$ KS/MO Waste Water

Pace Project No.: 60109211

\begin{tabular}{|c|c|c|c|c|c|c|}
\hline \multirow{3}{*}{$\begin{array}{l}\text { LABORATORY CONTROL SAMPLE: } \\
\text { Parameter }\end{array}$} & \multicolumn{6}{|l|}{905183} \\
\hline & & Spike & LCS & LCS & $\% \operatorname{Rec}$ & \\
\hline & Units & Conc. & Resull & $\% \operatorname{Rec}$ & Limits & Qualiflers \\
\hline Vinyl chloride & $\mathrm{ug} / \mathrm{L}$ & 20 & 17.5 & 88 & $57-163$ & \\
\hline Xylene (Total) & $\mathrm{ug} / \mathrm{L}$ & 60 & 53.9 & 90 & $75-120$ & \\
\hline 1,2-Dichloroethane-d4 (S) & $\%$ & & & 103 & $82-119$ & \\
\hline 4-Bromofluorobenzene (S) & $\%$ & & & 104 & $87-113$ & \\
\hline Dibromofluoromethane (S) & $\%$ & & & 101 & $86-112$ & \\
\hline Toluene-d8 (S) & $\%$ & & & 99 & $90-110$ & \\
\hline
\end{tabular}




\section{QUALITY CONTROL DATA}

Project: $\quad$ KS/MO Waste Waler

Pace Project No: $\quad 60109211$

\begin{tabular}{llll}
\hline QC Batch: & WETA 18128 & Analysis Method: & EPA 353.2 \\
QC Batch Method: & EPA 353.2 & Analysis Description: & 353.2 Nitrate + Nitrite, Unpres.
\end{tabular}

Associated Lab Samples: 60109211001

METHOD BLANK: $903260 \quad$ Matrix: Water

Associated Lab Samples: 60109211001

$\frac{\text { Parameter }}{\text { Nitrogen, Nitrate }}-\frac{\text { Units }}{\mathrm{mg} / \mathrm{L}}-\frac{\begin{array}{c}\text { Blank } \\ \text { Result }\end{array}}{\mathrm{ND}} \frac{\begin{array}{c}\text { Reporting } \\ \text { Limit }\end{array}}{0.10} \frac{\text { Analyzed }}{11 / 02 / 1108: 39} \frac{\text { Qualifiers }}{10}$

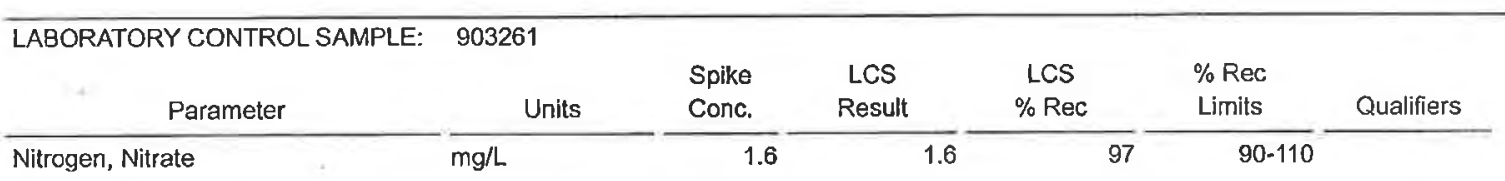

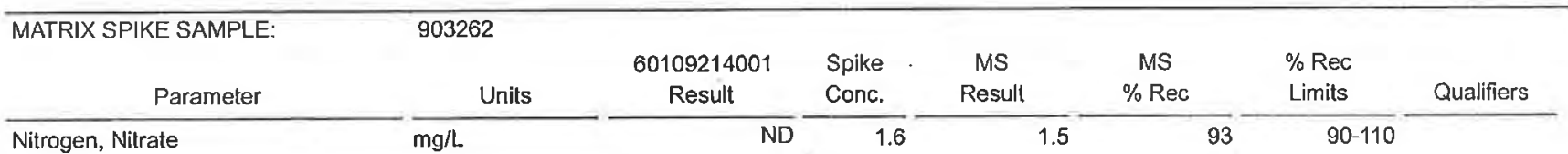

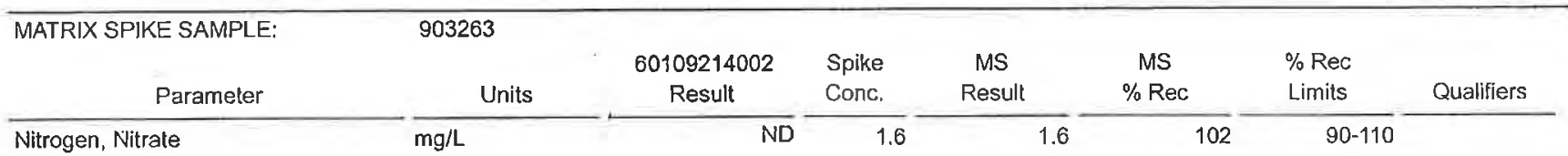

SAMPLE DUPLICATE: 903264
$\begin{gathered}\text { Parameter } \\ \text { Nitrogen, Nitrate }\end{gathered} \frac{\text { Units }}{\mathrm{mg} / \mathrm{L}} \frac{\begin{array}{c}60109214008 \\ \text { Result }\end{array}}{\text { ND }} \frac{\text { Result }}{\text { ND }} \frac{\text { RPD }}{\text { Rax }}$




\section{QUALITY CONTROL DATA}

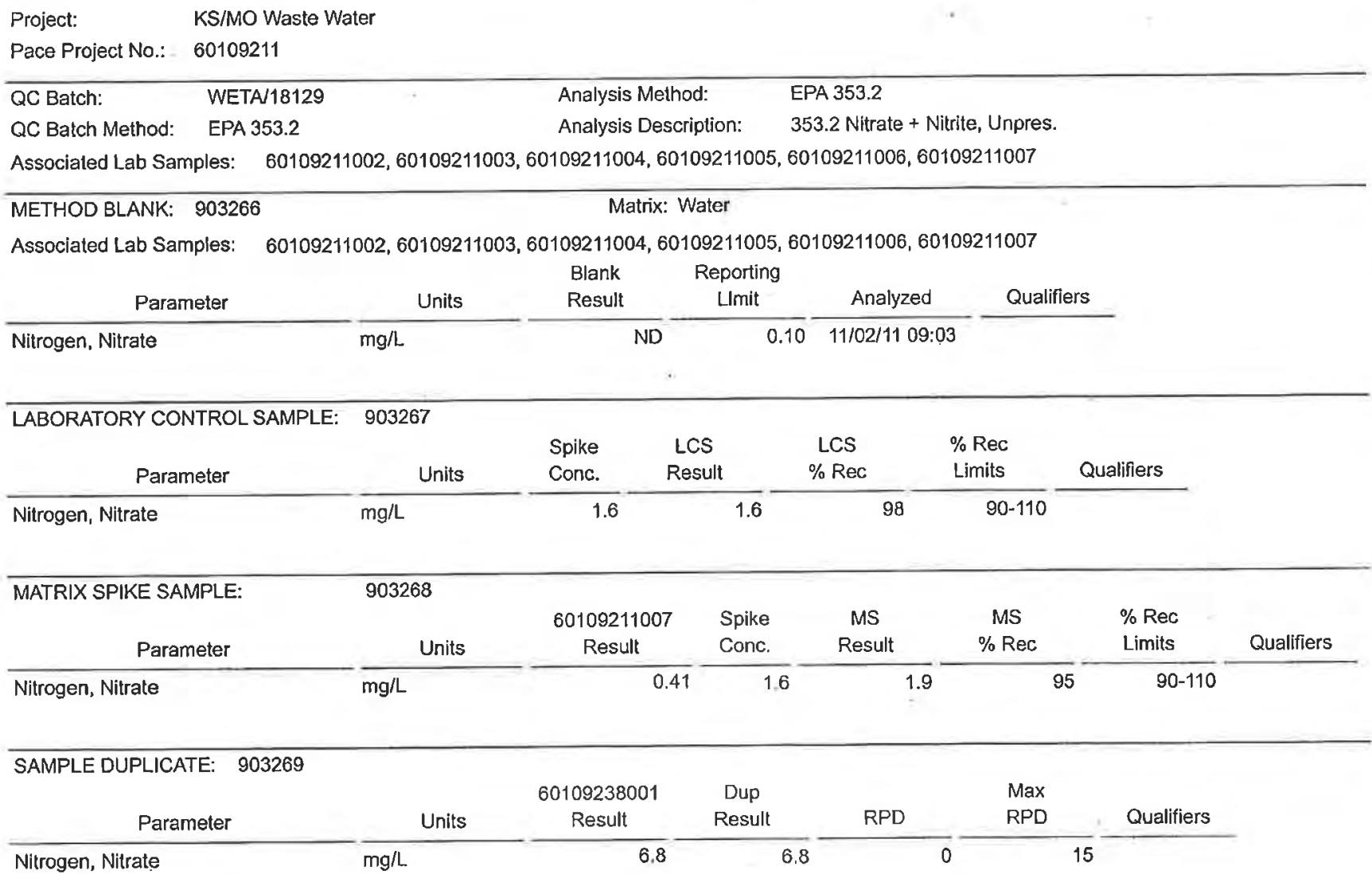




\section{QUALIFIERS}

Project: $\quad$ KS/MO Waste Water

Pace Project No.: 60109211

\section{DEFINITIONS}

DF - Dilution Factor, if reported, represents the factor applied to the reported data due to changes in sample preparation, dilution of the sample aliquol, or moisture content.

ND - Not Detected at or above adjusted reporting limit.

$\mathrm{J}$ - Estimated concentration above the adjusted method detection limit and below the adjusted reporting limit.

MDL - Adjusted Method Detection Limit.

S - Surrogate

1,2-Diphenylhydrazine (8270 listed analyte) decomposes to Azobenzene.

Consistent with EPA guidelines, unrounded data are displayed and have been used to calculate \% recovery and RPD values.

LCS(D) - Laboratory Control Sample (Duplicate)

MS(D) - Matrix Spike (Duplicate)

DUP - Sample Dupllcate

RPD - Relative Percent Difference

NC - Not Calculable.

SG - Silica Gel - Clean-Up

$U$ - Indicates the compound was analyzed for, but not detected.

N-Nitrosodiphenylamine decomposes and cannol be separated from Diphenylamine using Method 8270 . The result reporled for each analyte is a combined concentration.

Pace Analytical is TNI accredited. Contact your Pace PM for the current list of accredited analytes.

\section{BATCH QUALIFIERS}

Batch: MSV/41422

[M5] A matrix spike/matrix spike duplicale was not performed for this batch due to insufficient sample volume. 


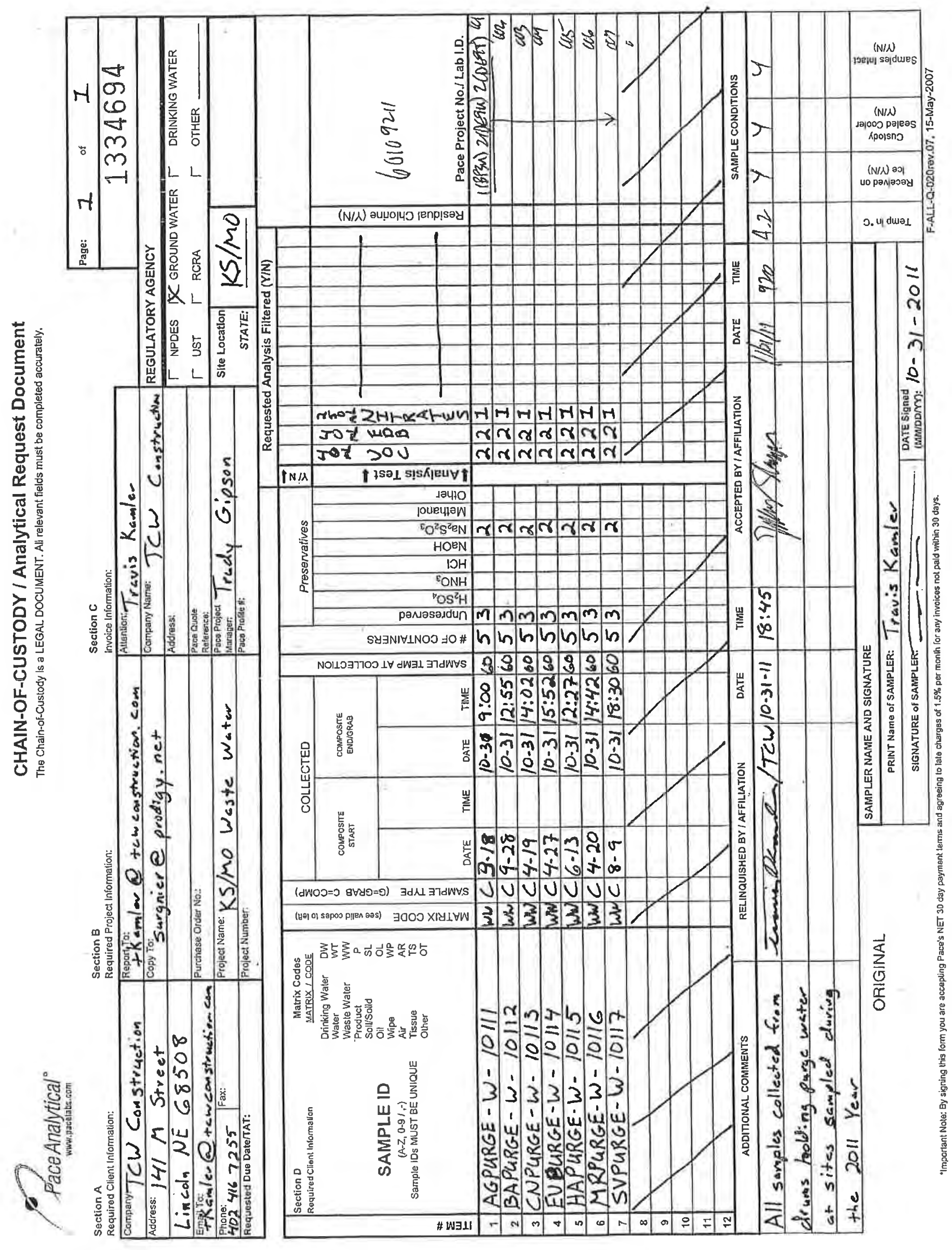




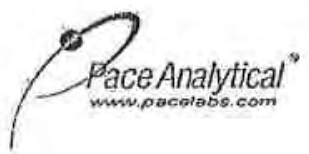

Sample Condition Upon Receipt

Client Name: TCW Const.

Project \# 601092-11

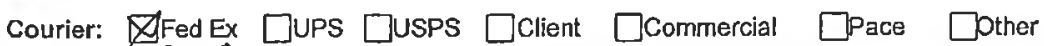
Tracking \#: 875827463563 Pace Shipping Label Used? $\square$ Yes $\otimes$ No

Custody Seal on Cooler/Box Present: $\bigotimes$ Yyes $\square$ No Seals intact: $\bigotimes$ Yes $\square$ No

\begin{tabular}{l}
\hline Optional \\
\hline $\begin{array}{l}\text { Proj. Due Date: } \\
\text { Proj. Name: }\end{array}$ \\
\hline
\end{tabular}

Packing Material: \女Bubble Wrap $\square$ Bubble Begs $\square$ Foam $\square$ None $\square$ pther

Thermometer Used: T-19P / T-194

Cooler Temperature: 4,2

Temperature should be above freezing to $6^{\circ} \mathrm{C}$

Type of Ice: Blue None

$\square$ Samples on ice, cooling process has begun

Comments:

Date and Initials of person examining

Chain of Custody present:

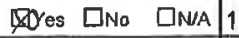

Chain of Custody filled out:

QTes DNo DNA 2.

Chain of Custody relinquished: ҰYes $\square$ No $\square \mathrm{N} / \mathrm{A} 3$

Sampler name \& signature on $\mathrm{COC}$ : GYYes DNo DNA 4.

Samples arrived withln holding time:

DYes DNo DNA 5.

Short Hold Time analyses $(<72 \mathrm{hr})$ : GYes DNo DN/A 6.

Rush Turn Around Time requested:

DYes GNo DN/A 7

Sufficient volume: $\quad$ DYes DNo DN/A 8 .

Correct containers used: $\quad$ ZYes $\square$ DNo DN/A 9 ,

-Pace containers used: $\square$ Yes $\square$ No DN/A

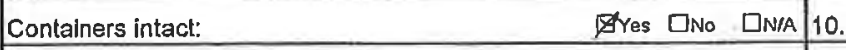

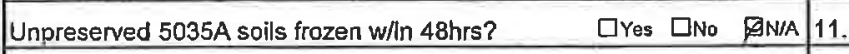

$\begin{array}{llll}\text { Filtered volume received for dissolved tests } \quad \square \text { Yes } & \text { 口No EQR/A } & 12 .\end{array}$

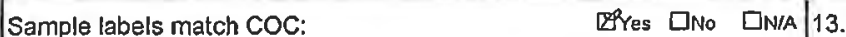

-Includes date/time/lD/analyses Matrix:

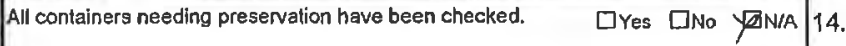

All containers needing preservation are found to be in $\square$ Yes $\square$ No G compliance with EPA recommendation.

Exceptons: VOA, colitorm, TOC, O\&G, WI-DRO (water), Phenolics

Trip Blank present:

QPYes DNo Initial when 1 Lot \# of added

Pace Trio Blank lot \# (if purchased):

Headspace in VOA vials (>6 $\mathrm{mm})$ :

¿Yes 区'No DN/A 15

$\mathrm{NO}_{3}$

contents: Dल $11 / 1 / h$ 1025

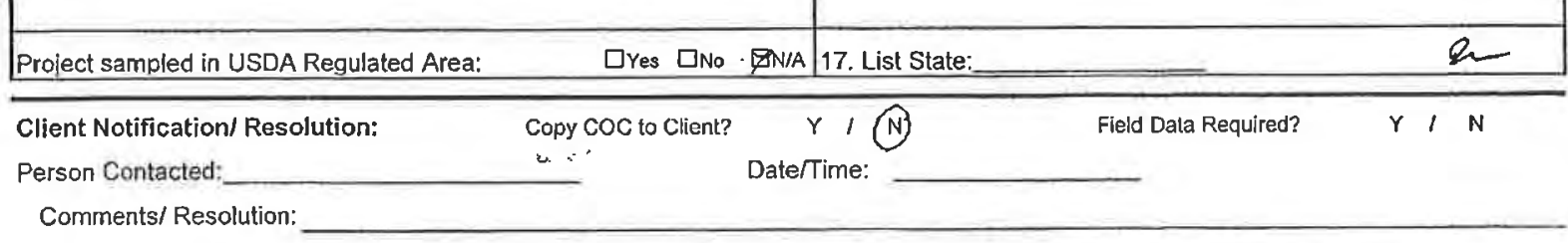

Project Manager Review:

Note: Whenever there is a discrepancy affecting North Carolina compliance samples, a copy of this form will be sent to the North Carolina DEHNR Certification Office ( i.e out of hold, incorrect preservative, out of temp, incorrect containers) 


\section{AGEM OI L}

CITY OF SABETHA Cash - Travis 805 MAIN

PO BOX 197

SABETHA KS 66534

$785 \cdot-284-2158$

Recolpt No: 2.015320

Dec 19, 2011

TCW Const.

WASTEWATER FUND-MISC

Purged Water

50.00

502-00.000-4632

MISCELLANEOUS INCOME

Total:

50.00

C'ash

50.00

Total Applled:

50.00

Change Tenderud:

50.00
50.00
50.00
.00
$.00=z== \pm= \pm=$


Supplement 2:

Sample Documentation from TestAmerica Laboratories, Inc., for Groundwater Verification Samples 


\section{TestAmerica}

THE LEADER IN ENVIRONMENTAL TESTING

\section{ANALYTICAL REPORT}

Job Number: 200-4827-1

SDG Number: 200-4827

Job Description: Morrill (200-4827)

Contract Number: EP-W-09-044

For:

Argonne National Laboratory

9700 South Cass Avenue

Building 203

Office B-149

Argonne, IL 60439

Attention: Mr. Clyde Dennis

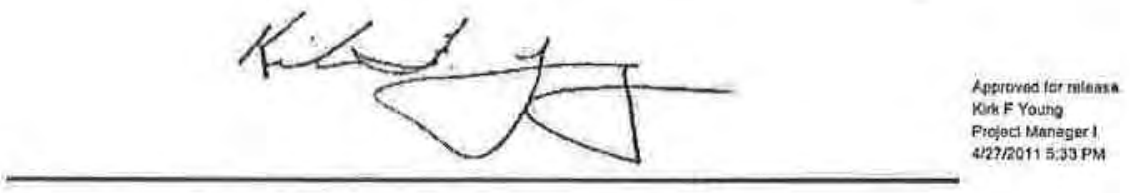

Kirk F Young

Project Manager I

kirk.young@testamericainc.com

04/27/2011

The test results in this report relate only to sample(s) as received by the laboratory. These test results were derived under a quality system that adheres to the requirements of NELAC. Pursuant to NELAC, this report may not be produced in full without written approval from the laboratory 


\section{Table of Contents}

Cover Title Page $\ldots \ldots \ldots \ldots \ldots \ldots \ldots \ldots \ldots \ldots \ldots \ldots \ldots \ldots, 1$

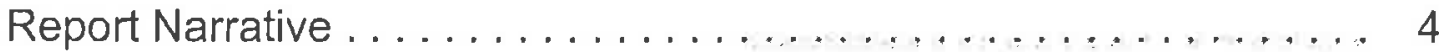

Case Narrative .............................. 4

Qualifier Definition ................................ 6

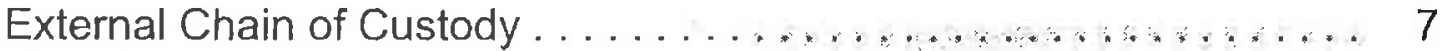

Internal Chain of Custody . . . . . . . . . . . . . . . . . . . . 8

Shipping Documentation . . . . . . . . . . . . . ......... 10

Airbills (if Applicable) . ................................. 11

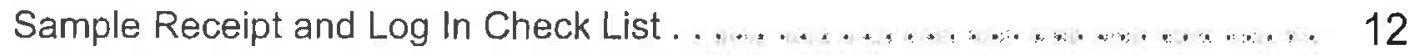

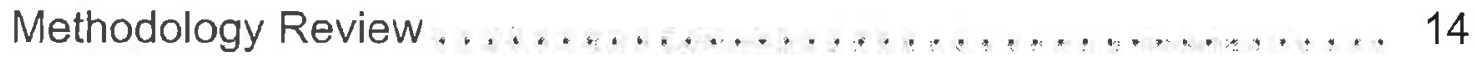

QC Summary - SOM01.2 Volatiles-Trace . . . . . . .............. 15

QC Summary - SOM01.2 Volatiles-Trace . ....................... 15

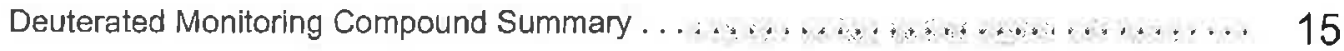

Method Blank . ...................................... 17

GC/MS instrument Performance Check ............................ 18

Internal Standard Area and RT Summary . .......................... 20

Sample Data - SOM01.2 Volatiles-Trace . . . . . . . . . . . . . . . . 21

Sample Data - SOM01.2 Volatiles-Trace ........................ 21

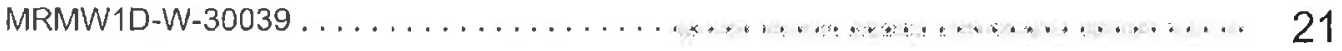

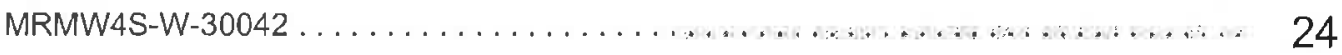

MRMW6S-W-30044 $\ldots \ldots \ldots \ldots \ldots \ldots \ldots \ldots \ldots \ldots \ldots, 27$

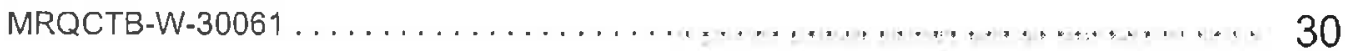

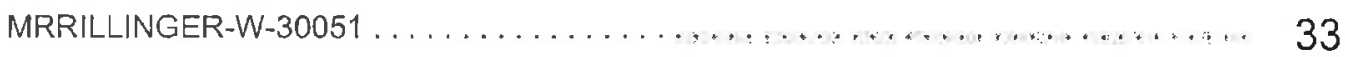

Standards - SOM01.2 Volatiles-Trace . . . . . . . . . . . . . . . 36

Standards - SOM01.2 Volatiles-Trace . ....................... 36

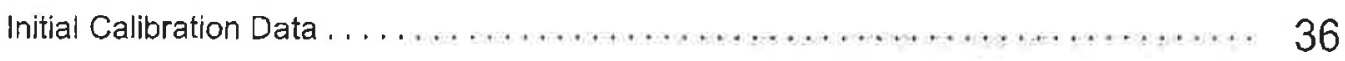




\section{Table of Contents}

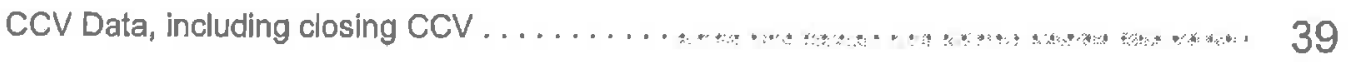

Raw Qc Data - SOM01.2 Volatiles-Trace . . . . . . . . . . . . . . . . . 45

Raw Qc Data - SOM01.2 Volatiles-Trace . . . . . . ....................... 45

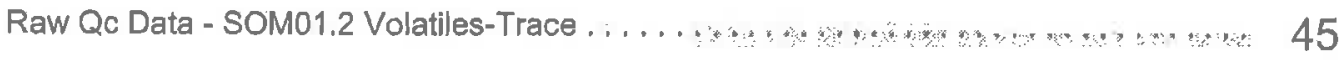

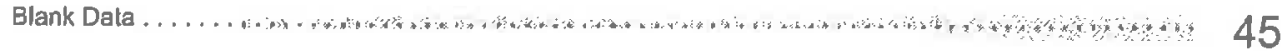




\title{
CASE NARRATIVE
}

\section{Client: Argonne National Laboratory}

\author{
Project: Morrill (200-4827)
}

\section{Report Number: $200-4827-1$}

\begin{abstract}
Enclosed is the data set for the referenced project work. With the exceptions noted as flags or footnotes, standard analytical protocols were followed in performing the analytical work and the applied control limits were met.
\end{abstract}

Calculations were performed before rounding to avoid round-off errors in calculated results.

All holding times were met and proper preservation noted for the methods performed on these sampies, unless otherwise detailed in the individual sections below.

\section{Receipt}

The samples were received on 04/22/2011. Documentation of the condition of the samples at the time of their receipt and any exception to the laboratory's Sample Acceptance Policy is documented in the Sample Handling section of this submittal. The samples, as received, were not acid preserved. On that basis, the laboratory did provide for the analytical work to be performed within seven days of sample collection.

\section{SOM01.2 Volatile Organics (Trace Level Water)}

A storage blank was prepared for volatile organics analysis, and stored in association with the storage of the samples. That storage blank, identified as VHBLK01, was carried through the holding period with the samples, and analyzed.

Each sample was analyzed without a dilution. Each of the analyses associated with the sample set exhibited an acceptable internal standard performance. There was an acceptable recovery of each deuterated monitoring compound (DMC) in the analysis of the method blank associated with the analytical work, and in the analysis of the storage blank associated with the sample set. The analysis of the samples in this sample set did meet the technical acceptance criteria specific to DMC recoveries, although not all DMC recoveries were within the control range in each analysis. The technical acceptance criteria does provide for the recovery of up to three DMCs to fall outside of the control range in the analysis of field samples. Matrix spike and matrix spike duplicate analyses were not performed on samples in this sample set. Trace concentrations of chloromethane, acetone, carbon disulfide, methylene chloride, trichloroethene, tolvene, 1,2,4-trichlorobenzene, and 1,2,3-trichlorobenzene were identified in the analysis of the method blank associated with the analytical work. The concentration of each compound in that analysis was below the established reporting limit, and the analysis did meet the technical acceptance criteria for a compliant method blank analysis. Trace concentrations of acetone and carbon disulfide were identified in the analysis of the storage blank associated with the sample set. The concentration of each compound in that analysis was below the established reporting limit, and the analysis did meet the technical acceptance criteria for a compliant storage blank analysis. Present in the method blank and storage blank analyses was a non-target constituent that represents a compound that is related to the DMC formulation. The fact that the presence of this compound is not within the laboratory's control is at issue. The derived results for that compound have been qualified with an " $X$ " qualifier to reflect the source of the contamination. 
The responses for each of the target analytes met the relative standard deviation criterion in the initial calibration. The response for each target analyte met the percent difference criterion in the opening/continuing calibration check acquisition. The response for each target analyte met the 50.0 percent difference criterion in the closing calibration check acquisition.

The primary quantitation mass for methylcyclohexane that is specified in the Statement of Work is mass 83 . The laboratory did identify a contribution to mass 83 from 1,2-dichloropropane- $d_{6}$, one of the deuterated monitoring compounds (DMCs). The laboratory did change the primary quantitation mass assignment to mass 55 for the quantification of methylcyclohexane.

Manual integration was employed in deriving certain of the analytical results. The values that have been derlved from manual integration are qualifled on the quantitation reports. Extracted ion current profiles for each manual integration are included in the data package, and further documented at the end of this submittal. 


\section{DATA REPORTING QUALIFIERS}

Client: Argonne National Laboratory

Job Number: $\quad 200-4827-1$

Sdg Number; 200-4827

Lab Section

Qualifier

Description

GC/MS VOA

U

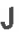

J

X

(

B

N
Analyzed for but not detected.

Indicates an Estimated Value for TICs

Indicates an estimated value.

See case narrative notes for explanation of the ' $X$ ' flag

Surogate exceeds the control limit

The analyte was found in an associated blank, as well as in the sample.

This flag indicates the presumptive evidence of a compound. 


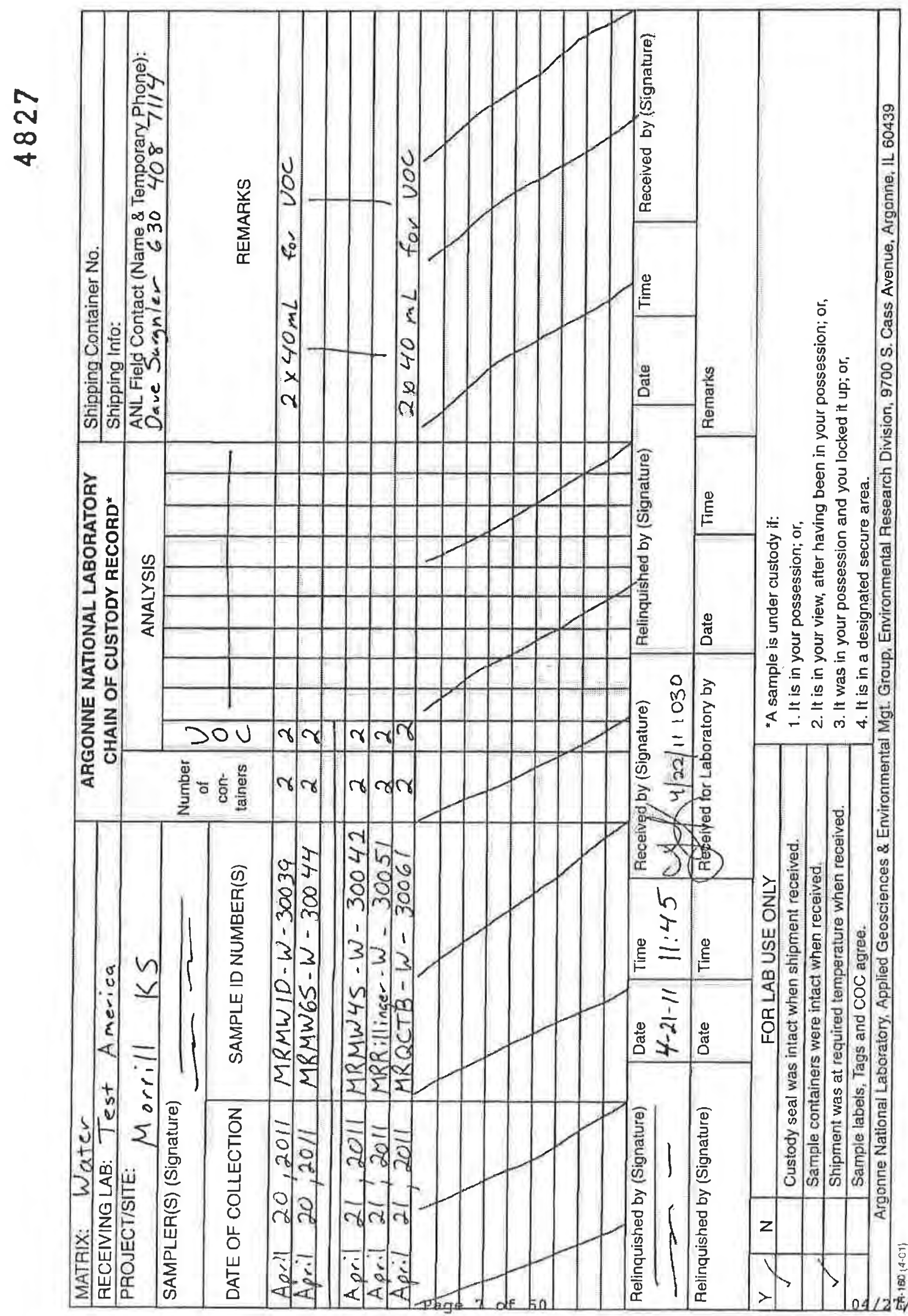




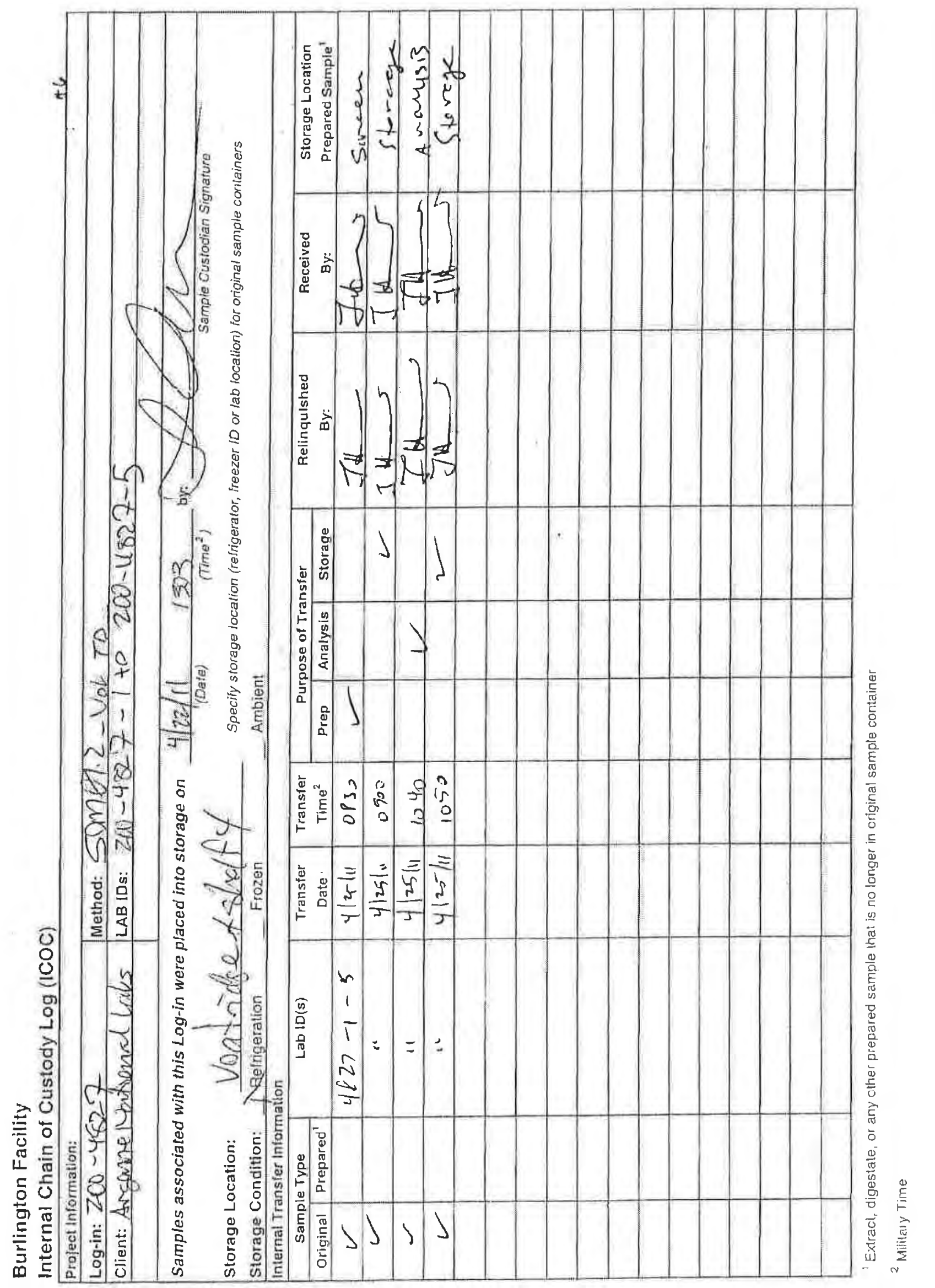




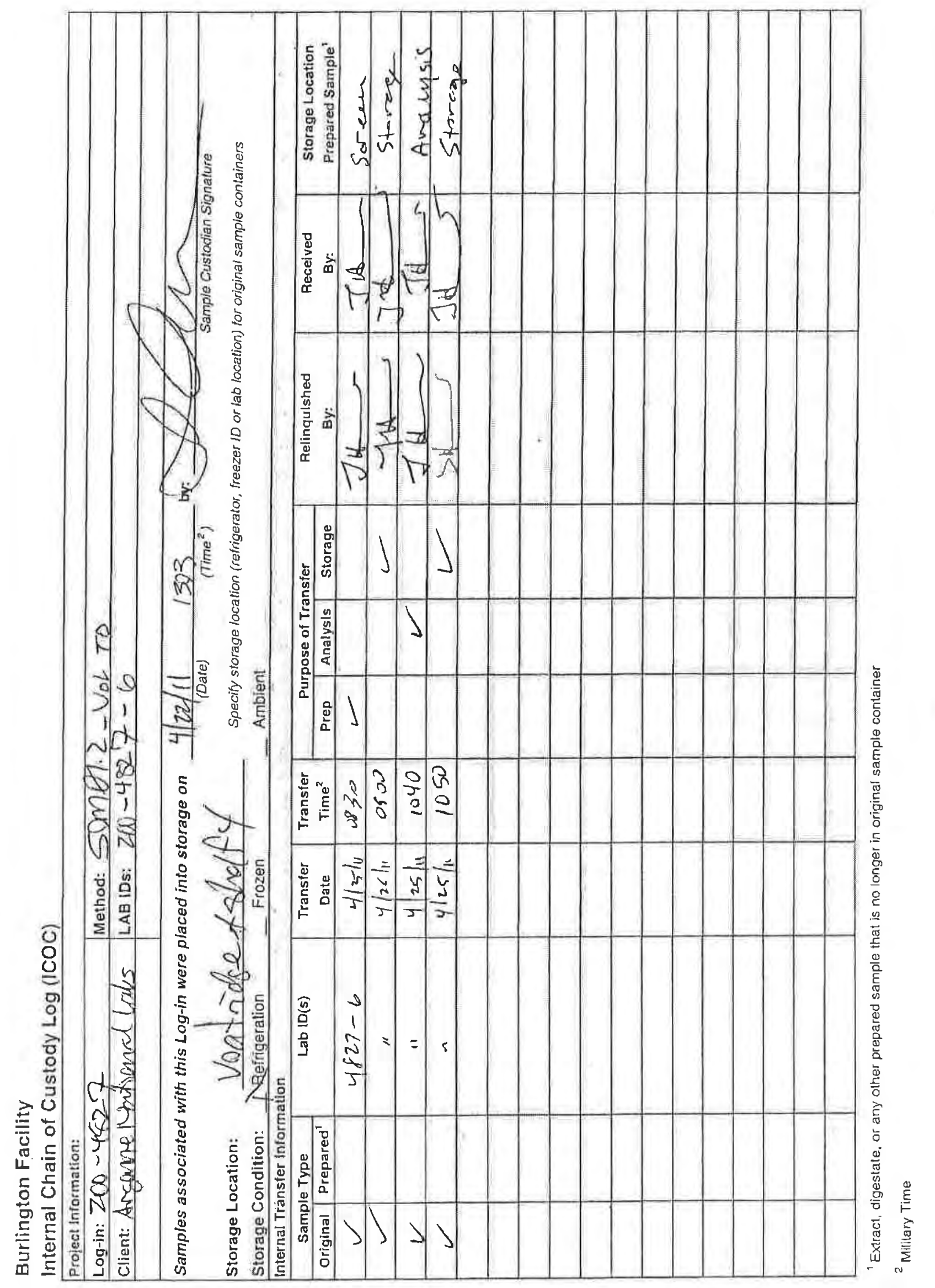




\section{Shipping and Receiving Documents}




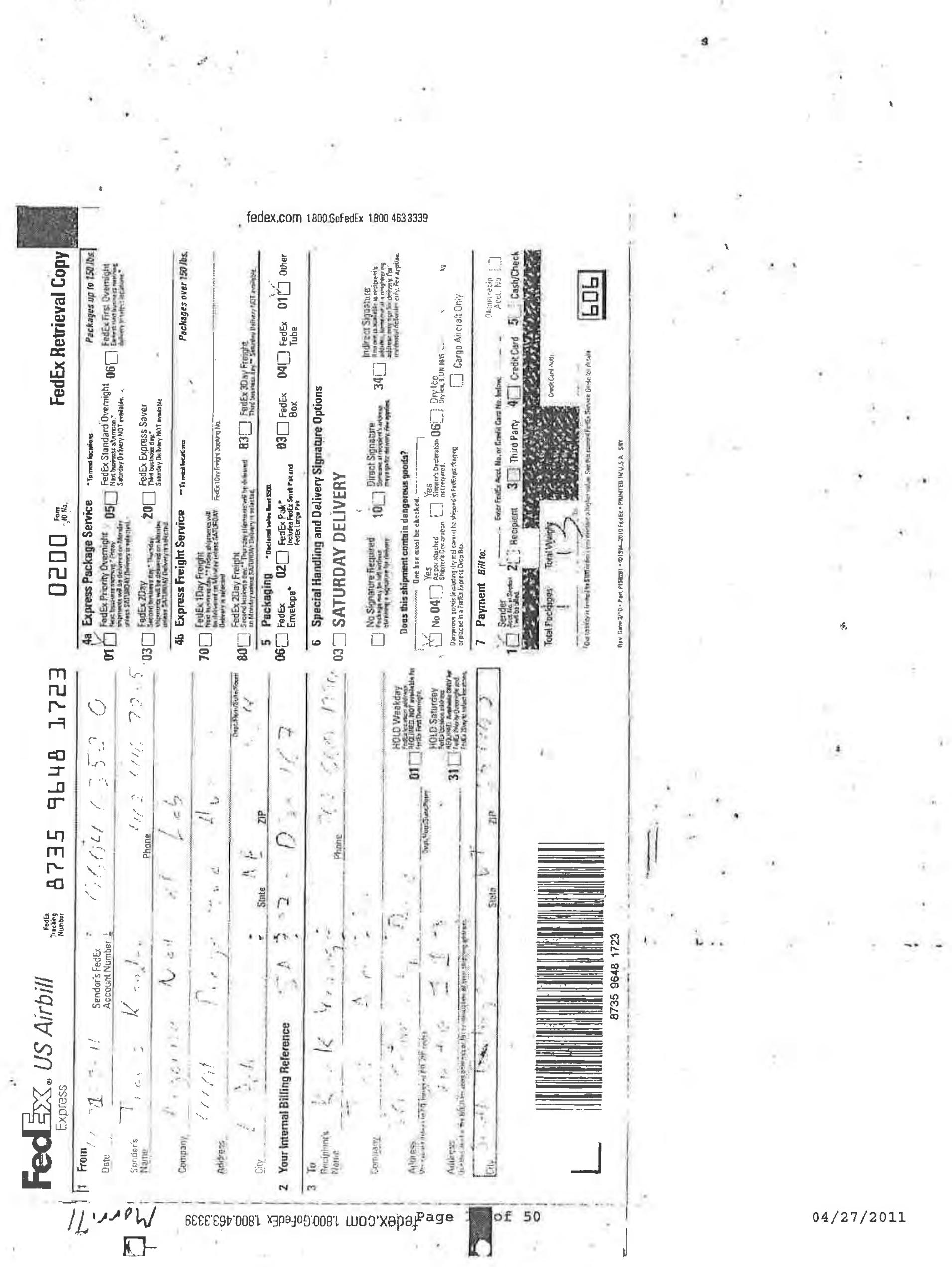




\section{Login Sample Receipt Checklist}

Client: Argonne National Laboratory

Job Number: $200-4827-1$

SDG Number: $200-4827$

Login Number: 4827

List Source: Testamerlca Burlington

List Number: 1

Creator: Marion, Greg T

Question

Radioactivity either was not measured or, if measured, is at or below background

The cooler's custody seal, if present, is intact.

The cooler or samples do not appear to have been compromised or tampered with.

Samples were received on ice.

Cooler Temperature is acceptable.

Cooler Temperature is recorded,

$\mathrm{COC}$ is present.

$\mathrm{COC}$ is filled out in ink and legible.

$\mathrm{COC}$ is filled out with all pertinent information.

Is the Field Sampler's name present on COC?

There are no discrepancies between the sample IDs on the containers and the COC.

Samples are received within Holding Time.

Sample containers have legible labels.

Containers are not broken or leaking.

Sample collection date/times are provided.

Appropriate sample containers are used.

Sample bottles are completely filled.

Sample Preservation Verified.

There is sufficient vol, for all requested analyses, incl. any requested MS/MSDs

VOA sample viais do not have headspace or bubble is $<6 \mathrm{~mm}(1 / 4 ")$ in diameter.

Multiphasic samples are not present.

Samples do not require splitting or compositing.

Residual Chlorine Checked.
Answer

N/A
True
True
True
True
True
True
True
True
True
True
True
True
True
True
True
True
N/A
True
True
N/A
N/A
N/A

Comment 


\section{Sample Login Acknowledgement}

Job 200-4827-1

$\begin{array}{ll}\text { Cllent Job Descriptlon: } & \text { Morrill (200-4827) } \\ \text { Purchase Order \#: } & 8 \mathrm{E}-00302 \\ \text { Work Order \#: } & \text { 8E-00302 } \\ \text { Project Manager: } & \text { Kirk F Young } \\ \text { Job Due Date: } & 5 / 6 / 2011 \\ \text { Job TAT: } & 14 \text { Days } \\ \text { Max Deliverable Level: } & \mathrm{N} \\ & \\ \text { Earliest Deliverable Due: } & 5 / 6 / 2011\end{array}$
Report To: Argonne National Laboratory
Jorge Alvarado
9700 South Cass Avenue
Building 203
Office B-149
Argonne, IL 60439
BIII To: Argonne National Laboratory
Accounts Payable
Chief Financial Offices
9700 S. Cass Ave.
Building 201
Argonne, IL 60439

\section{Login 200-4827}

\begin{tabular}{|c|c|}
\hline Sample Receipt: & $4 / 22 / 2011 \quad 10: 30: 00 \mathrm{AM}$ \\
\hline lethod of Dellvery: & iority Overnight \\
\hline $\begin{array}{l}\text { Lab Sample \# } \\
\text { Method }\end{array}$ & $\begin{array}{l}\text { Client Sample ID } \\
\text { Method Description / Work Location }\end{array}$ \\
\hline $200-4827-1$ & MRMW1D-W-30039 \\
\hline SOM01.2_Vol_Tr & SOM01.2 Trace Volatile Orga \\
\hline $0-4827-2$ & MRMW6S-W-30044 \\
\hline SOM01.2_Vol_Tr & Ir SOM01.2 Trace Volatile Or \\
\hline $200-4827-3$ & MRMW4S-W-3 \\
\hline SOM01.2_Vol_Tr & SOM01;2 Tr \\
\hline $200-4827-4$ & MRRILLINGER-W-30051 \\
\hline SOM01.2_Vol_Tr & SOM01.2 Trace Volatile Organics / In-Lab \\
\hline \multirow{2}{*}{$\begin{array}{l}\text { 200-4827-5 } \\
\text { SOM01.2_Vol_Tr }\end{array}$} & MRQCTB-W-30061 \\
\hline & SOM01.2 Trace Volatile Organics / In-La \\
\hline \multicolumn{2}{|c|}{ 200-4827-6 VHBLK01 } \\
\hline SOM01.2_Vol_Tr & SOM01.2 Trace Volatile Organics / In-La \\
\hline
\end{tabular}

Number of Coolers: $\quad 1$

Cooler Temperature(s) $\left(\mathrm{C}^{\circ}\right): 2.9$;

\begin{tabular}{|c|c|c|c|}
\hline \multirow[t]{2}{*}{ Date Sampled } & \multirow[t]{2}{*}{ Matrix } & & \\
\hline & & Rpt Basis & Dry / Wet ** \\
\hline \multirow[t]{2}{*}{ 4/20/2011 12:00:00 AM } & Water & & \\
\hline & & Total & Wet \\
\hline \multirow[t]{2}{*}{ 4/20/2011 12:00:00 AM } & Water & & \\
\hline & & Total & Wet \\
\hline 4/21/2011 12:00:00 AM & Water & & \\
\hline & & Total & Wet \\
\hline 4/21/2011 12:00:00 AM & Water & & \\
\hline & & Total & Wet \\
\hline 4/21/2011 12:00:00 AM & Water & & \\
\hline & & Total & Wet \\
\hline \multirow[t]{2}{*}{$4 / 22 / 2011 \quad 12: 45: 00 \mathrm{P}$} & Water & & \\
\hline & & Total & Wet \\
\hline
\end{tabular}




\section{METHODOLOGY SUMMARY}

\begin{tabular}{|c|c|c|c|}
\hline Laboratory: & TestAmerica Laboratories & Project No: & \\
\hline Location: & South Burlington, Vermont & SDG No: & $200-4827$ \\
\hline
\end{tabular}

VOA

Volatile Organics Trace - USEPA CLP SOM01.2 
$2 A$ - FORM II VOA-1

WATER VOLATILE DEUTERATED MONTTORTNG COMPOUND RECOVERY

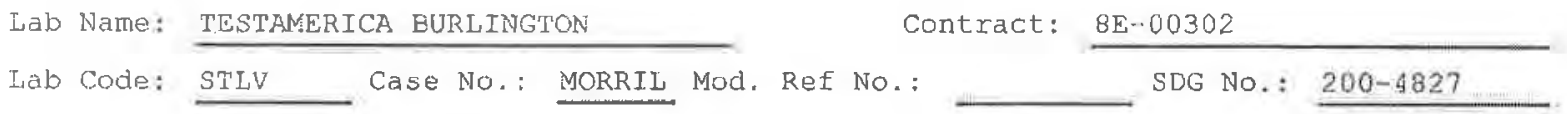

Level: (TRACE or INOW) IRACE

\begin{tabular}{|c|c|c|c|c|c|c|c|}
\hline $\begin{array}{c}\text { EPA } \\
\text { SAMPIE NO. }\end{array}$ & $\begin{array}{l}\text { VDHCI } \\
\text { (VCE) \# }\end{array}$ & $\begin{array}{l}\text { VDMC2 } \\
(\mathrm{CLA})\end{array}$ & $\begin{array}{l}\text { VDMC3 } \\
\text { (DCE) \# }\end{array}$ & $\begin{array}{l}\text { VDMCA } \\
\text { (BUT) \# }\end{array}$ & $\begin{array}{l}\text { VDMCS } \\
\text { (CLE) } \#\end{array}$ & $\begin{array}{l}\text { VDMC6 } \\
\text { (DCA) \# }\end{array}$ & $\begin{array}{l}\text { VDMC7 } \\
\text { (BEN) \# }\end{array}$ \\
\hline VBEKJZ & 90 & 90 & 75 & 105 & 102 & 114 & 102 \\
\hline MRMW 1 D-W-30039 & 85 & 84 & 69 & 153 & 99 & 109 & 97 \\
\hline MRMW 6S-W-30044 & 79 & 81. & 66 & 153 & 92 & 107 & 91 \\
\hline MRMW $4 S-W-30042$ & 79 & 32 & 69 & 250 & 96 & 107 & 93 \\
\hline $\begin{array}{l}\text { MRRILLFNGER-W- } \\
30051\end{array}$ & 81 & 82 & 67 & $166 \%$ & 95 & 109 & $\overline{9} \overline{3}$ \\
\hline MRQCTE-W-3006I & 80 & 79 & 68 & $156 \%$ & 91 & 1.03 & 91 \\
\hline VHBLKO1 & 76 & 79 & 66 & 85 & 90 & 100 & 91 \\
\hline
\end{tabular}

VDMCl $\quad\left(V C I_{1}\right)=$ Vinyl Chloride-d3

VDMC2 (CLA) = Chloroethane $-\mathrm{d} 5$

VDMC3 $(D C E)=1,1-D i c h l o r o e t h e n e-d 2$

VDMCA (BUT) = 2-Butanone-a.5

VDMC5 (CIF) = Chlocoform-d

VDMC6 $(D C A)=1,2-$ Dichloroethane-d4

VDMC7 (BEN) = Benzene-di

$$
\begin{array}{r}
\text { QC LTMITS } \\
\hline(65-131) \\
(71-131) \\
(55-104) \\
(19-155) \\
(78-121) \\
(78-129) \\
(77-124)
\end{array}
$$

\# Colum to be used to fläg recovery values

* Values outside of contract requirea QC Iimits 
$2 B$ - FORM II VOA-2

WATER VOLATILE DEUTERATED MONITORING COHPOUND RECOVERY

Lab Name: TESTAMERICA BURLINGTON

Contract: $8 E-00302$

\begin{tabular}{|c|c|c|c|c|c|c|c|c|}
\hline $\begin{array}{c}\text { EPA } \\
\text { SAMPLE No. }\end{array}$ & $\begin{array}{l}\text { VDMC8 } \\
\text { (DFA) \# }\end{array}$ & $\begin{array}{l}\text { VDMC9 } \\
\text { (TOL) \# }\end{array}$ & $\begin{array}{l}\text { VDMC10 } \\
\text { (TDP) \# }\end{array}$ & $\begin{array}{l}\text { VDMC11 } \\
\text { (HEX) \# }\end{array}$ & $\begin{array}{l}\text { VDMC12 } \\
\text { (TCA) \# }\end{array}$ & $\begin{array}{l}\text { VDMC13 } \\
\text { (DCZ) if }\end{array}$ & OTHER & $\begin{array}{l}\text { TOT } \\
\text { OUT }\end{array}$ \\
\hline VBLKIZ & 92 & 106 & 110 & 111 & 104 & 114 & & 0 \\
\hline MRMWID-W-30039 & 87 & 101 & 109 & $182 *$ & 104 & 108 & & 1 \\
\hline MRMW $6 S-W-30044$ & 85 & 95 & 103 & $173 *$ & .100 & 100 & & 1 \\
\hline MRMWAS-W-30042 & 84 & 96 & 103 & $171 *$ & 98 & 106 & & 1 \\
\hline $\begin{array}{l}\text { MRRILIINGER-W- } \\
30051\end{array}$ & 85 & 96 & 107 & $184 *$ & 99 & 105 & & 2 \\
\hline MRQCTB-W-30061 & 82 & 95 & 99 & 178 * & 93 & 101 & & 2 \\
\hline VHBLK01 & 81 & 96 & 100 & 98 & 91 & 100 & & 0 \\
\hline
\end{tabular}

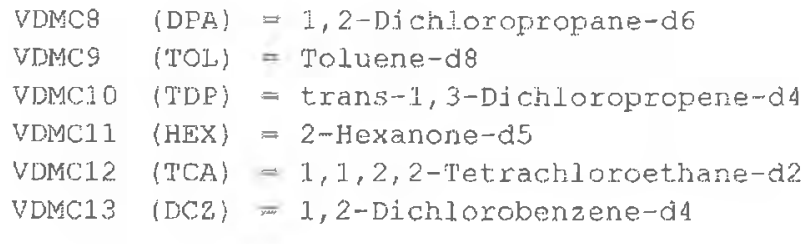


$4 A$ - FORM IV VOA

VOLATTLE METHOD BLANK SUMMARY
EPA SAMPLE NO.

VBLKJZ

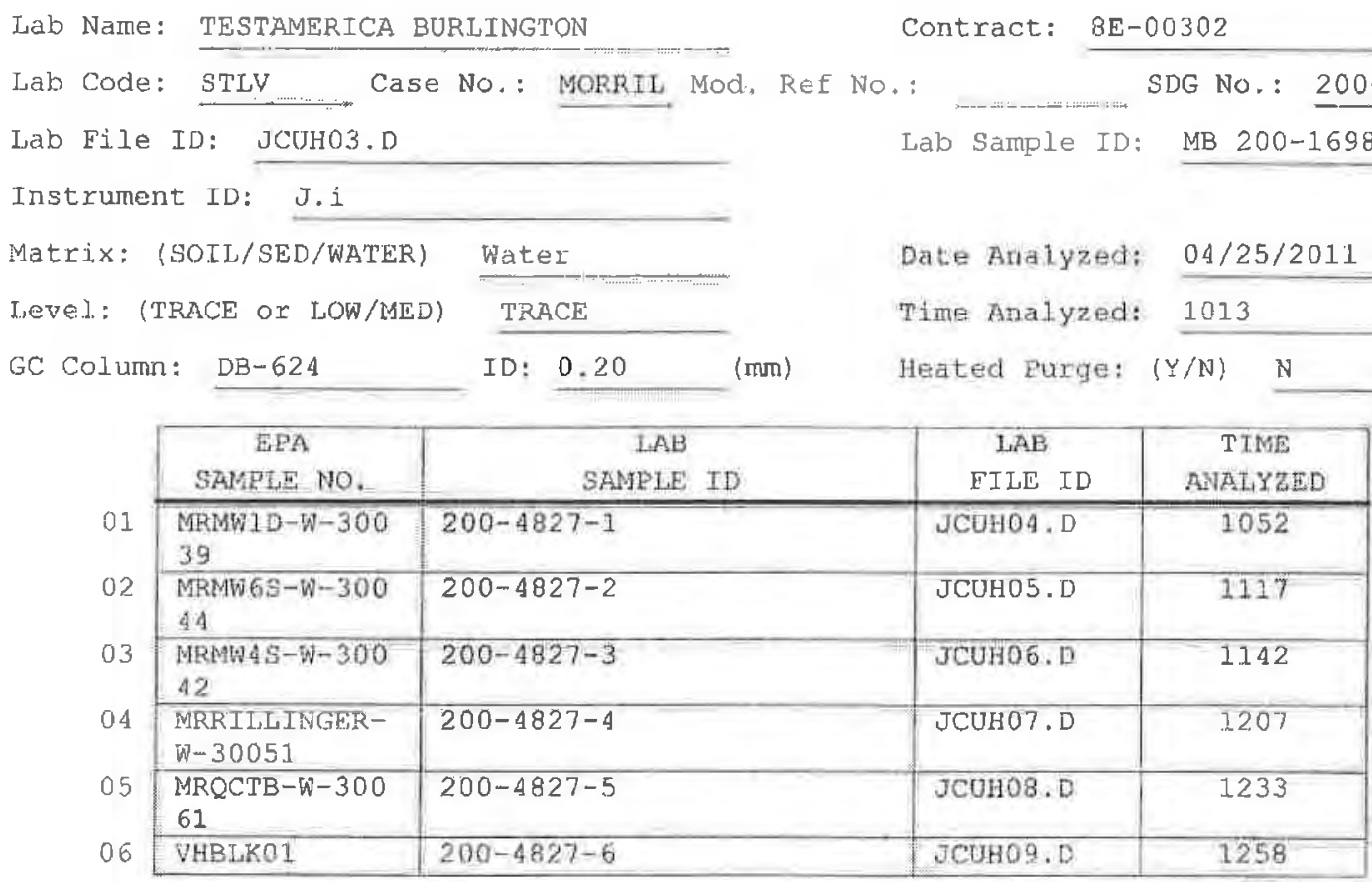

COMMENTS: 
$5 A$ - FORM V VOA

VOLATILE ORGANICS INSTRUMENT

PERFORMANCE, CHECK

BROMOFT,UOROBENZENE (BFB)
EPA. SAMPLE NO. BEBJR
Läb Name; TESTAMERICA BURLINGTON

Lab Code: STLV

Lab File Id: JCu01.

Instrument Id: J.i

GC Colurn: DB-624 Case No.: MORRYL MOd. Ref No.:
Contract: $\quad 8 E-00302$

SDG No.: $200-4827$

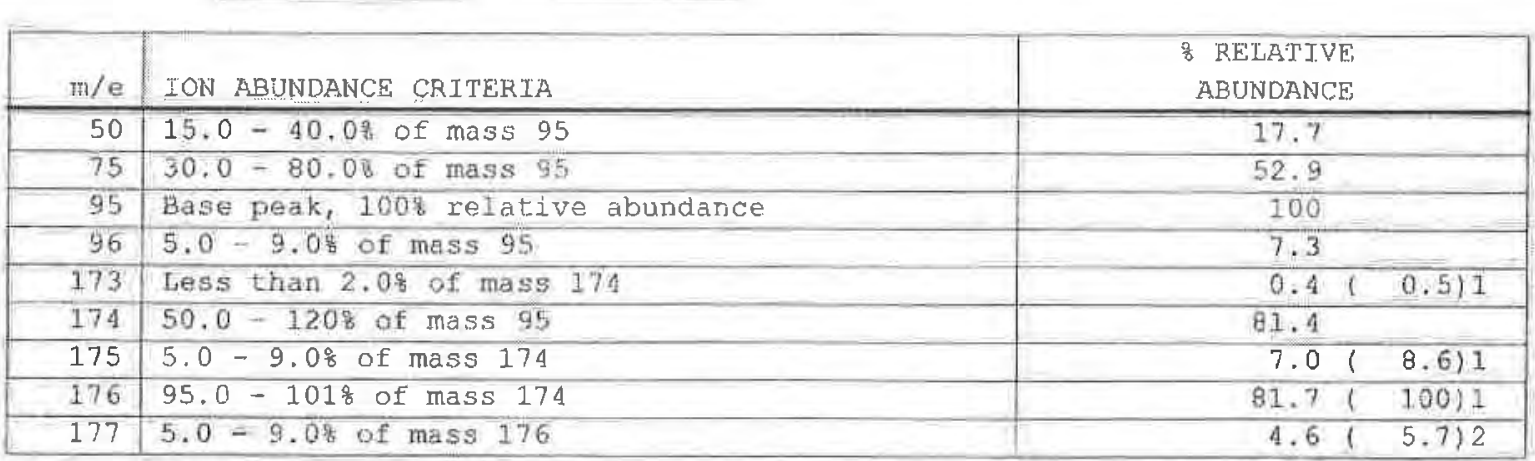

1. - Value is omass 174

2 - Value is gamass 176

\begin{tabular}{|c|c|c|c|c|}
\hline $\begin{array}{c}\text { EPA } \\
\text { SAMFLE NO. }\end{array}$ & $\begin{array}{c}\text { LAB } \\
\text { SAMPLE ID }\end{array}$ & $\begin{array}{c}\text { LAB } \\
\text { FTLE ID }\end{array}$ & $\begin{array}{c}\text { DATE } \\
\text { ANALYZED }\end{array}$ & $\begin{array}{c}\text { TTME } \\
\text { ANALYZED }\end{array}$ \\
\hline VSTD0.5JR & IC $200-15618 / 3$ & JCU03.D & $03 / 24 / 2011$ & 1409 \\
\hline VSTD001JR & IC $200-\cdots 1561874$ & JCU04.D & $03 / 24 / 2011$ & 1433 \\
\hline VSTD005JR & ICIS $200-15618 / 5$ & zCU05.D & $03 / 24 / 2011$ & 1459 \\
\hline VSTDO10JR & IC $200-15618 / 6$ & JCU06.D & $03 / 24 / 2011$ & 1524 \\
\hline VSTDD020JR & IC $200-15618 / 7$ & JCU07.D & $03 / 24 / 2011$ & 1549 \\
\hline
\end{tabular}


$5 A-F O R M$ V VOA

VOLATILE' ORGANTCS INSTRUMENT:

PERFORMANCE CHECK

BROMOFLUOROBENZENE (BFB)
EPA SAMPLE NO. BFBJZ
Lab Name: 'TESTAMERICA BURLINGT'ON Lab Code: STLV_ Case No.: MORRIL Mod, Ref No.:

Lab File Id: JCUH01.D

Instrument Id: J.i

GC Colimin: DB-624

ID: 0.20
Contract: $8 \mathrm{E}-00302$

SDG NO.: 200-4827

BFB Injection Date: 04/25/20j1

BFB Injection Tjme: 0928 (mm)

\begin{tabular}{|c|c|c|}
\hline $\mathrm{m} / \mathrm{e}$ & ION PBUNDANCE CRITERIA & $\begin{array}{r}\text { RELATIVE } \\
\text { ABUNDANCE }\end{array}$ \\
\hline 50 & $15.0-40.0$ 号 of thass 95 & 16.6 \\
\hline 75 & $30.0-80.05$ of mass 95 & 44.6 \\
\hline 95 & Base peak, 1000 relative abundance & 100 \\
\hline 96 & $5.0-9.08$ of mass 95 & 6.9 \\
\hline 173 & Less than $2.0 \%$ of mass 1.74 & 0.31 \\
\hline 174 & $50.0-1208$ of mass 95 & 92.3 \\
\hline 175 & $5.0-9.0 \%$ of mass 174 & 7.51 \\
\hline 176 & $95.0-101$ 은 of mass 174 & $89.1(96.6) 1$ \\
\hline 177 & $5.0-9.08$ of mass 176 & $6.4(7.1) 2$ \\
\hline
\end{tabular}

1 - Value is somass 174

2 - Value is imass 176

\begin{tabular}{|c|c|c|c|c|}
\hline $\begin{array}{c}\text { EPA } \\
\text { SAMPLE NO. }\end{array}$ & $\begin{array}{c}\text { IAB } \\
\text { SAMPIJE ID }\end{array}$ & $\begin{array}{l}\text { LAB } \\
\text { FILE ID }\end{array}$ & $\begin{array}{c}\text { DATE } \\
\text { ANALYZED }\end{array}$ & $\begin{array}{c}\text { TIME } \\
\text { ANALYZED }\end{array}$ \\
\hline$\overline{\text { VSTD005JZ }}$ & CCVIS 200-16989/2 & JCUH02.D & $04 / 25 / 2011$ & 0948 \\
\hline VBLKJZ & MB 200-16989/3 & JCUH03.D & $04 / 25 / 2011$ & 1013 \\
\hline $\begin{array}{l}\text { MRMW } 1 D-W-3 \\
0039\end{array}$ & $200-4827-1$ & JCUHO4.D & $04 / 25 / 2011$ & 2052 \\
\hline $\begin{array}{l}\text { MRMW 6S-W-3 } \\
0044\end{array}$ & $200-4827-2$ & JCUH05.D & $04 / 25 / 2011$ & 1117 \\
\hline $\begin{array}{l}\text { MRMW } 4 S-W-3 \\
0042\end{array}$ & $200-4827-3$ & JCUHO6.D & $04 / 25 / 2011$ & 1142 \\
\hline $\begin{array}{l}\text { MRRILIIINGE } \\
R-W-30051\end{array}$ & $200-4827-4$ & J̈CUH07.D & $04 / 25 / 2011$ & 1207 \\
\hline $\begin{array}{l}\text { MRQCTB- } w-3 \\
0051\end{array}$ & $200-4827-5$ & JCUH08.D & $04 / 25 / 2011$ & 1233 \\
\hline VHBLKOI & $200-4827-6$ & JCUH09.D & $04 / 25 / 2011$ & 1258 \\
\hline VSTD005ZJ & CCVC $200-16989 / 14$ & JCUH.14.D & $04 / 25 / 2011$ & 1507 \\
\hline
\end{tabular}


$8 A$ - FORM VIII VOA

VOLATITE INTERNAL STANDARD AREA AND RE'IENIION TIME SUMMARY

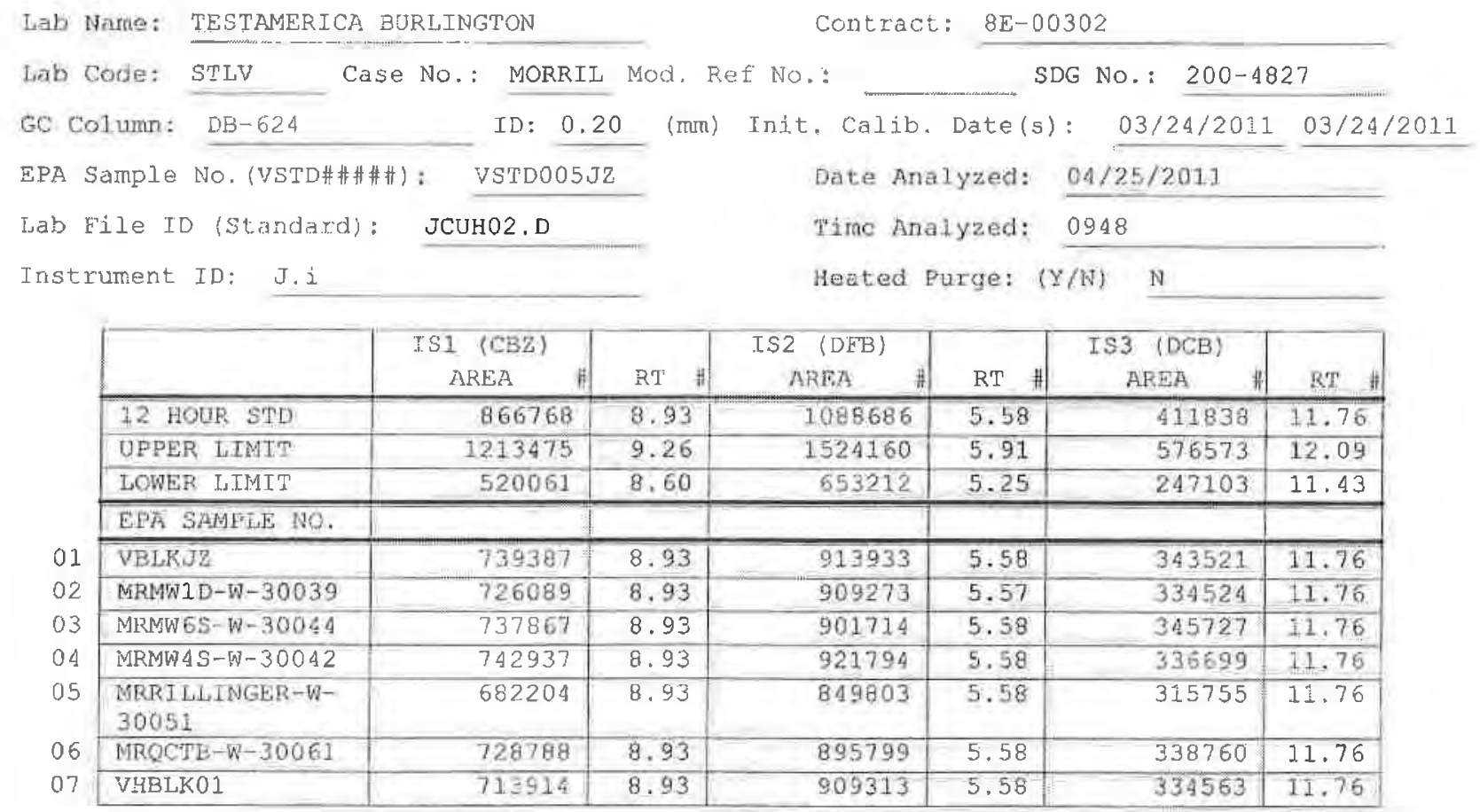

ISI (CBZ) = Chlorobenzene-d5

ISz (DFR) $=1,4-D$.ifluorobenzene

IS3 $(D C B)=1,4$-Dichlorobenzene-dA

AREA URPER LIMIT $=140$ (TxaCe Volatiles) of internal standard area AREA LOWER LIMIT = 60z (Trace Volatjles) of internal standard area

RT UPFER LIMIT $=+0.33$ (Trace Volatiles) minutes of internal standard RT.

RT LOWER LTMIT $=-0.33$ (Trace Volatiles) minutes of internal standard RT

\# Colum used to flag values outside contract required QC limits with an asterisk. 
$1 A$ - FORM I VOA-1

VOLATILE ORGANICS ANALYSIS DATA SHEET
EPA SAMPLE NO.

MRMW1D-W-30039

Lab Näme: TESTAMERICA BURLINGTON

Contract: $\quad$ BE-00302

Lab Code: STLV Case No.: MORRIL Mod. Ref No.: SDG No.: $200-4827$

Matri : (SOTL/SED/WATER)

Water

Sample wt/vol: 25.0 $(g / m L) \quad m i L$

Level: (TRACE/LOW/MED) TRACE.

s Moisture: not dec.

GC Colums: DB-624 ID: 0.20 (mum)

Soil Extract Volume: (uL) (nL) ab Sample ID: 200-4827-1

Lab File ID: JCUHO4,D

Date Received: $04 / 22 / 2011$

Date Analyzed: 04/25/2011

Dilution Factor: 1.0

Soli.l A.liquot Volume: (uL)

\begin{tabular}{|c|c|c|c|}
\hline CAS NO. & COMPOUND & $\begin{array}{l}\text { CONCENTRATION UNITS: } \\
(\mathrm{ug} / \mathrm{L} \text { or } \mathrm{ug} / \mathrm{kg}) \mathrm{ug} / \mathrm{L}\end{array}$ & $\mathrm{Q}$ \\
\hline $75-71-8$ & Dichiorodifluoromethane & 0.50 & U \\
\hline $74-87-3$ & Chloromethane & 0.50 & $\mathrm{U}$ \\
\hline $75-01-4$ & Vinyi chloride & 0.50 & $\mathrm{U}$ \\
\hline $74-83-9$ & Bromometrane & 0.50 & U \\
\hline $75-00-3$ & Crijoxoethane & 0.50 & $\mathrm{U}$ \\
\hline $75-69-4$ & Trichorofluoromethane & 0.50 & 0 \\
\hline $75-35-4$ & 1,1-Dichloroethene & 0.50 & U \\
\hline $76-13-1$ & $1, i, 2-T^{\prime} i c h l o r o-i, 2,2-t r i f l$ uoroethane & 0.50 & U \\
\hline $67-64-1$ & Acetone & 0.60 & J $B$ \\
\hline $75-15-0$ & Carbon disulfide & 0.16 & J B \\
\hline $79-20-9$ & Methyl acetate & 0.50 & 0 \\
\hline $75-09-2$ & Methyleane Chloride & 0.50 & $\mathrm{U}$ \\
\hline $156-60-5$ & trans-1,2-Dichioroethene & 0.039 & $J$ \\
\hline $1634-04-4$ & Methyl tert-butyl ether & 0.50 & U \\
\hline $75-34-3$ & 1,1-Dichloroethane & 0.50 & U \\
\hline $156-59-2$ & cis-1,2-Dichloroethene & 0.50 & $\mathrm{U}$ \\
\hline $78-93-3$ & 2-Butamone & 5.0 & t) \\
\hline $74-97-5$ & Bromochloramethare & 0.50 & 0 \\
\hline $67-66-3$ & Chloroform: & 0.50 & $\mathrm{U}$ \\
\hline $71-55-6$ & $1,1,1$-Trichloroethane & 0.50 & [] \\
\hline $1.10-82-7$ & Cyclohexane & 0.50 & $\mathrm{U}$ \\
\hline $56-23-5$ & Carbon tetrachloride & 0.022 & $\mathrm{~J}$ \\
\hline $71-43-2$ & Benzene & 0.50 & U \\
\hline $107-06-2$ & 1,2 -Dichloroethane & 0.50 & E \\
\hline
\end{tabular}


$1 B$ - FORM I VOA-2

VOIATILF ORGANECS ANALYSTS DA'A SHEE'I
EPA SIMPLE: NO.

VRMW1D-W-30039

Lab Name: TESTAMERICA BURLINGTON

Contract: $\quad 8 \mathrm{E}-00302$

Lab code: STLV Case No.: MORRIL Mod. Rest No.: SDG NO.: 200-4827

Matrix: (SOIL/SED/WATER) Water

Sample wt/vol: $25.0(\mathrm{~g} / \mathrm{mL}) \mathrm{mL}$

Level: (TRACE/LOW/MED) TRACE

c Moisture: not dec.

GC Column: DB-624 ID: 0.20 (mn)

Thम

Soil Extract Vo] urne:

Purge Volume: 25.0
I:ab Sampie ID: 200-4827-1

Lab Eile ID: JCUHO4.D

Date Received: 04/22/2011

Date Analyzed: $04 / 25 / 2011$

Di]lition Factor: 1.0

Soil Mliquot Volume:

(uL)

\begin{tabular}{|c|c|c|c|}
\hline CAS NO. & COMPOUND & $\begin{array}{l}\text { CONSENTRATION UNITS: } \\
(\mathrm{ug} / \mathrm{I} \text { or } \mathrm{ug} / \mathrm{kg}) \mathrm{ug} / \mathrm{L}\end{array}$ & $Q$ \\
\hline $79-01-6$ & "Trichloroethene & 0.50 & $\mathrm{U}$ \\
\hline $108-87-2$ & Methylcyclohexane & 0.50 & U \\
\hline $78-87-5$ & 1,2-Dichloropropane & 0.50 & $\mathrm{U}$ \\
\hline $75-27-4$ & Eromodichloromethane & 0.50 & U \\
\hline $10061-01-5$ & cis-1,3-Dichloropropene & 0.50 & U \\
\hline $108-10-1$ & 4-Methy]-2-pentanore & 5.0 & $\mathrm{U}$ \\
\hline $108-88-3$ & Toluene & 0.021 & J $\mathrm{B}$ \\
\hline $10061-02-6$ & trans $-1,3$-Dichloropropene & 0.50 & U \\
\hline $79-00-5$ & $1,1,2-$ Trichioroethane & 0.50 & $\mathrm{U}$ \\
\hline $127-18-4$ & Tetrachloroethene & 0.50 & $\mathrm{U}$ \\
\hline $591-78-6$ & 2-Hexánone & 5.0 & $\mathrm{U}$ \\
\hline $124-48-1$ & Dibromochloromethane & 0.50 & D) \\
\hline $106-93-4$ & 1,2-Dibromoethane & 0.50 & U \\
\hline $108-90-7$ & Chlorobenzene & 0.50 & U \\
\hline $100-41-4$ & Ethylbenzerie & 0.013 & J \\
\hline $95-47-6$ & o-xylene & 0.50 & U \\
\hline $179601-23-1$ & m, $p-x y l$ lene & 0.052 & $\mathrm{~J}$ \\
\hline $100-42-5$ & styrene & 0.50 & U \\
\hline $75-25-2$ & D.romotcrm & 0.50 & $\mathrm{U}$ \\
\hline $93-82-8$ & Isopropylbenzene & 0.50 & [ ] \\
\hline $79-34-5$ & $1,2,2,2$-I'etrachloroe thane & 0.50 & U \\
\hline $541-73-1$ & 1,3-Dichlorobenzene & 0.50 & $\mathrm{U}$ \\
\hline $106-46-7$ & 1,4-Dicllorobenzone & 0.50 & $\mathrm{U}$ \\
\hline $95-50-1$ & 1,2-Dichlorobenzene & 0.50 & $\mathrm{U}$ \\
\hline $96-12-8$ & 1,2-Dibromo-3-Chloropropalle & 0.50 & $\mathrm{U}$ \\
\hline $220-82-1$ & 1,2,4-Prichlorobenzene & 0.50 & U \\
\hline $87-61-6$ & 1,2,3-Trichloroberzene & 0.50 & U \\
\hline
\end{tabular}




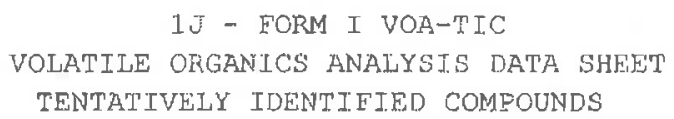

EPA SAMPLE NO. TENTATIVELY IDENTIFIED COMPOUNDS

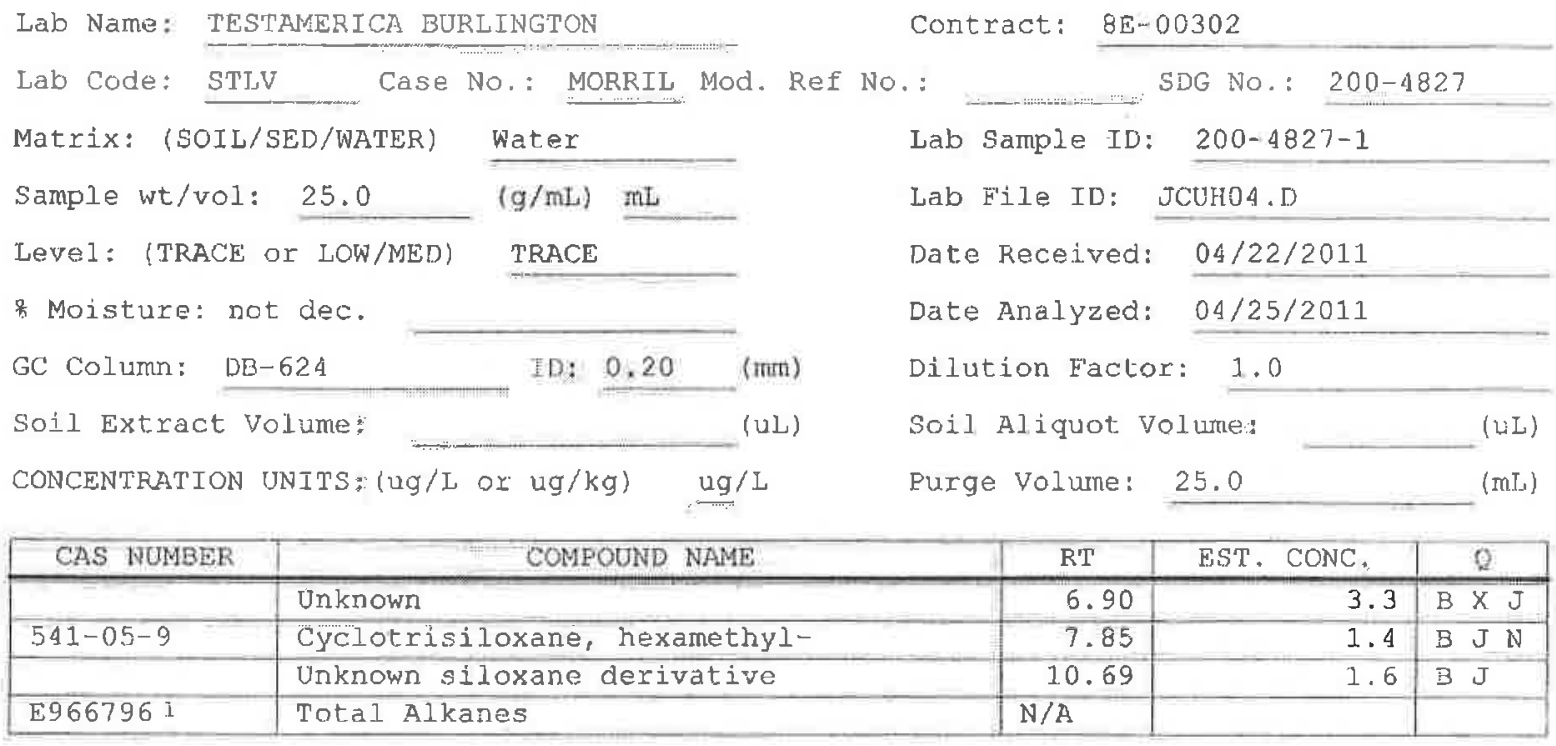

1 EPA-designated Registry Number. 
$1 A$ - FORM I VOA-1

VOLATILE ORGANICS ANALYBIS DATA SHEET
EPA SAMPLE NO.

MRMW $45-W-30042$

Lab Nane: TESTAMERICA BURLINGTON

Contract: $\quad 8 \mathrm{E}-00302$

Lab Cocie: STJV Case No.: MORRIJ Mod. Ref No.: SDG NO.: $200-4827$

Matrix: (SOIL/SED/WATER)

Sample wt/vol: 25.0 Water

$(\mathrm{g} / \mathrm{m} \mathrm{m}, \mathrm{mL}$

LeVE L: (TRACE/LOW/MED) TRACF

\% Moisture: not dec.

GC Columin: DB-624 ID: 0,20 (Inm)

Soil Extract Volume: (uL) (mL)

Purge volume: 25.0
Lab Samole ID: $\quad 200-4827-3$

Lab File ID: JCUHO6.D

Date Received: $04 / 22 / 2011$

Date Analyzed: 04/25/2011

Dilution Factor: 1, O

soil Aliguot Volume:

(uL)

\begin{tabular}{|c|c|c|c|}
\hline CAS NO. & COMPOUND & $\begin{array}{l}\text { CONCENTRATION UNITS: } \\
(\mathrm{ug} / \mathrm{L} \text { or ug/kos } \mathrm{ugg} / \mathrm{L}\end{array}$ & Q \\
\hline $75-71-8$ & Dichlorodifluoromethane & 0.50 & $\mathrm{U}$ \\
\hline $75-87-3$ & Chloromethane & 0.50 & U \\
\hline $75-01-4$ & Vinyl chloride & 0.50 & U \\
\hline $74-83-9$ & BIornomethane & 0.50 & U \\
\hline $75-00-3$ & Chloroethane & 0.50 & U \\
\hline $75-69-4$ & Trichlorofluoromethane & 0.50 & $\mathrm{U}$ \\
\hline $75-35-4$ & 1,1-Dichloroetnene & 0.50 & U \\
\hline $76-13-1$ & 1,1,2-Trichloto-1,2,2-trifluoroethane & 0.50 & $\mathrm{U}$ \\
\hline $67-64-1$ & Acetone & 1.1 & $\bar{J} B$ \\
\hline $75-1.5-0$ & Carbon disulfide & 0.083 & $\mathrm{~J} \mathrm{~B}$ \\
\hline $79-20-9$ & Wethyl acesate & 0.50 & $\overline{\mathrm{u}}$ \\
\hline $75-09-2$ & Methylere Chloride & 0.50 & $\mathrm{U}$ \\
\hline $156-60-5$ & trans-1,2-Dichloroethene & 0.50 & $\mathrm{U}$ \\
\hline $1634-04-4$ & Methyl tert-butyl ether & 0.50 & $\mathrm{U}$ \\
\hline $75-3 \Delta-3$ & 1,1-Dichloroethane & 0.50 & $\mathrm{U}$ \\
\hline $156-59-2$ & cis-1,2-Dichloroethene & 0.50 & $\mathrm{U}$ \\
\hline $78-93-3$ & 2-Butanorie & 5.0 & U \\
\hline $74-97-5$ & Bromochloromethane & 0.50 & $\mathrm{U}$ \\
\hline $67-66-3$ & Chloroform & 0.50 & $\mathrm{U}$ \\
\hline $71-55-6$ & 1,1,1-Trichloroethare & 0.50 & U \\
\hline $110-82-7$ & Cyclohexane & 0.50 & U \\
\hline $56-23-5$ & Carbon tetrachloride & 0.15 & $\mathrm{~J}$ \\
\hline $71-43-2$ & Benzene & 0.50 & I] \\
\hline $107-06-2$ & 1,2-Dichloroethane & 0.50 & U \\
\hline
\end{tabular}

Report 1, 4-Dioxane for Low-Medium VOA analysis ont 
$1 \mathrm{E}$-. FORM I $\mathrm{VOA}-2$

VOLATILE' ORGANICS ANAJXSTS ГATA SHFET
EFA SAMELE NO.

MRPW $4 S-W-30042$
Iab Name; TESTAMERICA BURLINGTON

lab Code: STIV__ Case No.: MORRIL Mod. Ref No.:
Water

$(\mathrm{g} / \mathrm{mL}) \mathrm{mL}$

Sample wt/vol: 25.0

TFACE

Level: (TRACE/LOW/MED)

응 Moisture: not dec.

GC Column: DB-624

ID: 0.20

Solil Extract Volume:

Purge Volume: 25.0

Contract: $\quad 8 E-00302$

SDG No.: $200-4827$

Iab Sample ID: $\quad 200-4827-3$

Lab File ID: JCUH06.D

Date Recoived: 04/22/2011

Date Arialyzed: Dấ/25/2011

Dilution Factor: 1.0

Soit $A]$ iqnol: Vollume:

(UL)

(ml)

\begin{tabular}{|c|c|c|c|}
\hline CAS NO. & COMPOUND & $\begin{array}{l}\text { CONCENIRFTION UNITS: } \\
(\mathrm{ug} / \mathrm{L} \text { OE } \mathrm{ug} / \mathrm{kg}) \mathrm{ug} / \mathrm{L}\end{array}$ & $Q$ \\
\hline $79-01-6$ & Trichlofoethene & 0.50 & $\mathrm{U}$ \\
\hline $108-87-2$ & Methylcyclohexane & 0.50 & U \\
\hline $78-87-5$ & 1,2-Dichloropropane & 0.50 & D \\
\hline $75-27-4$ & Bromodichloromethane & 0.50 & U \\
\hline $.10061-01-5$ & cis-1,3-Dichloropropene & 0.50 & 0 \\
\hline $108-10-1$ & 4-Methyl-2-pentanone & 5.0 & $\mathrm{U}$ \\
\hline $108-8 B-3$ & Toluene & 0.029 & $\bar{J} \bar{B}$ \\
\hline $10061-02-6$ & Erans-1, 3-Dichloropropene & 0.50 & U \\
\hline $79-00-5$ & 1.1,2-Trichloroethane & 0.50 & $\mathrm{U}$ \\
\hline $127-18-4$ & Tetrachloroethene & 0.50 & $\mathrm{j}$ \\
\hline $591-78-6$ & 2-Hexanone & 5.0 & U \\
\hline $1 \overline{2} 4-48-1$ & Dibromochioromethane & 0.50 & $\mathrm{U}$ \\
\hline $106-93-4$ & 1,2-Dibrompethane & 0.50 & U \\
\hline $108-90-7$ & Chlorobenzene & 0.50 & [] \\
\hline $100-41-1$ & Ethylbenzene & 0.0081 & $\mathrm{~J}$ \\
\hline $95-47-6$ & o-xylere & 0.50 & U] \\
\hline $179601-23-I$ & $\pi l, \mathrm{p}-\mathrm{Xyiene}$ & 0.035 & $\mathrm{~J}$ \\
\hline $100-42-5$ & styrene & 0.50 & $\mathrm{U}$ \\
\hline $75-25-2$ & Bromoformi & 0.50 & $\mathrm{U}$ \\
\hline $98-82-8$ & Isopropyibenzene & 0.50 & $\mathrm{U}$ \\
\hline $79-31-5$ & 1, 1, 2, 2-Tetzachloroethane & 0.50 & U \\
\hline $541-73-1$ & 1,3-[izchlorobenzene & 0.50 & [1 \\
\hline $106-46-7$ & 1, 1-Dichlorobenzene & 0.50 & $\mathrm{U}$ \\
\hline $95-50-1$ & 1,2-Djchlorobenzene & 0.50 & ij \\
\hline $96-12-2$ & 1,2-Dibromo-3-Chloropropane & 0.50 & L \\
\hline $120-82-1$ & $1,2,4$-Trichlorobenzene & 0.50 & $\mathrm{U}$ \\
\hline $87-61-6$ & 1, 2, 3-Trichlorobenzene & 0.50 & $\mathrm{U}$ \\
\hline
\end{tabular}


$1 \mathrm{~J}$ - FORM I VOA-TIC

VOLATILE ORGANICS ANALYSIS DATA SHEET TENTATIVELY IDENTIEIED COMPOUNDS
EPA SAMPLE NO.

MRMW 4S-W-30042

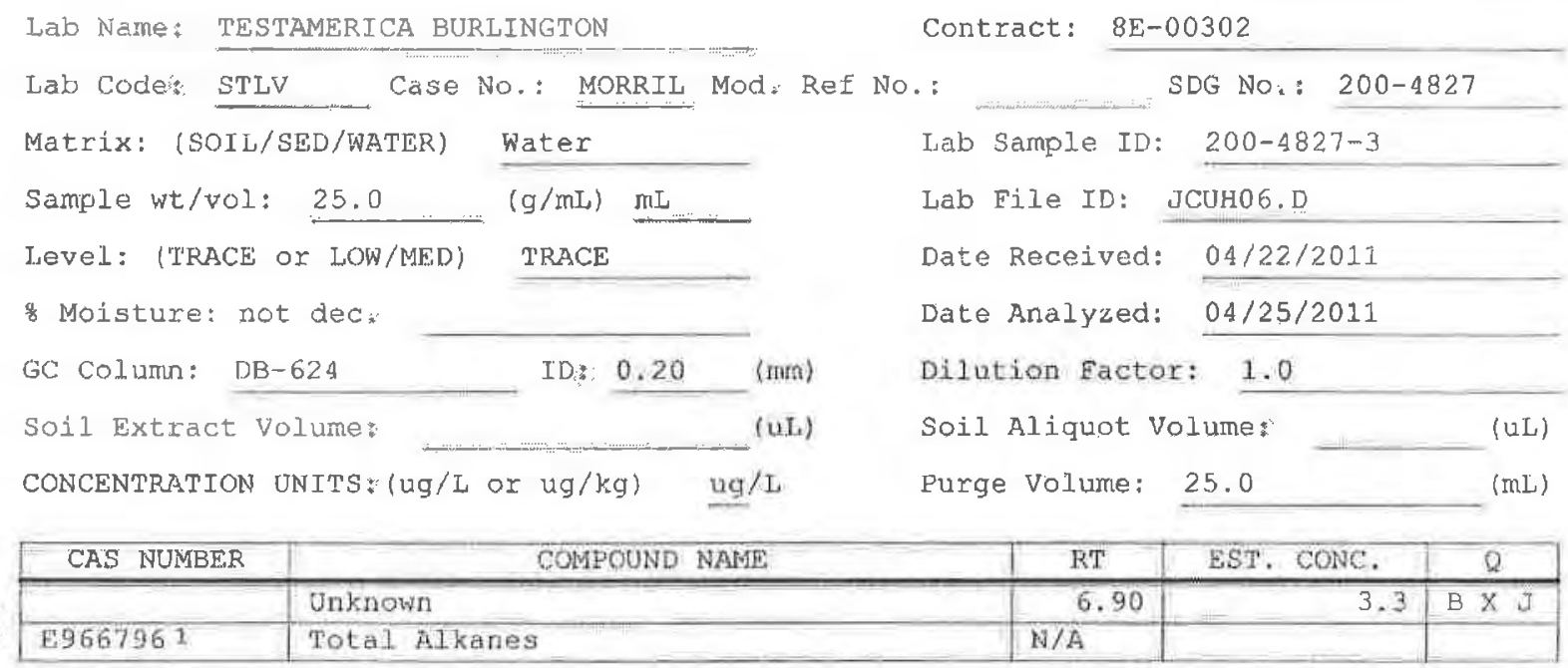

1 EPA-designated Registry Number. 
Lab code: STIV Case No.: MORRTL Mod, Ref. No.: SDG No, : 200-4827

Matrix: (SOIL/SED/WATER)

Sample wt/vol: 25.0 water

$(g / m L) \quad m L$

Level: (TRACE/LOW/MED) TRACE

\& Moisture: not dec.

GC Columin: $\quad \mathrm{DB}-624$

Soil Extract volume:

Purge Volume: 25.0

$\frac{\text { ID: } 0.20 \quad \text { (mm) }}{\text { ID: }}$

( $u T$, )

(mL)
Lab sampie ID* 200-4827-2

I.ab File ID: ICUHOS.D

Date Received: $04 / 22 / 2011$

Date Analyzed: $04 / 25 / 2011$

Dilution Factor: 1.0

Soil Aliquot Volume:

(ul)

\begin{tabular}{|c|c|c|c|}
\hline CAS NO. & COMPOUND & $\begin{array}{l}\text { CONCENTRATION UNITS: } \\
\text { (ug/L Or } \mathrm{ug} / \mathrm{kg} \text { ) ug/L }\end{array}$ & $Q$ \\
\hline $75-71-8$ & Dichlorodifluoromethane & 0.50 & U \\
\hline $74-87-3$ & Chloromethane & 0.50 & y \\
\hline $75-01-4$ & Vinyl chloride & 0.50 & $\mathrm{U}$ \\
\hline $74-83-9$ & Bromomethane & 0.50 & U \\
\hline $75-00-3$ & Chloroethane & 0.50 & $\mathrm{U}$ \\
\hline $75-69-4$ & Irichlorofluoromethane & 0.50 & U \\
\hline $75-35-4$ & 1,1-Dichloroethene & 0.50 & $\mathrm{U}$ \\
\hline $76+13-1$ & 1, 1,2-Trichloro-1,2,2-trifluorcetinane & 0.50 & U \\
\hline $67-64-1$ & Acetone & 1.1 & JE \\
\hline $75-15-0$ & Carbon disulfide & 0.098 & J $\mathrm{B}$ \\
\hline $79-20-9$ & Nethyl acetate & 0.50 & $\mathrm{U}$ \\
\hline $75-09-2$ & Methylene Chloride & 0.50 & 0 \\
\hline $156-60-5$ & trans-1,2-Dichloroethene & 0.50 & $\mathrm{U}$ \\
\hline $1634-04-4$ & Methyl tert-butyl ethex: & 0.50 & $\bar{U}$ \\
\hline $75-34-3$ & 1,1 -Dichloroethane & 0.50 & $\mathrm{U}$ \\
\hline $156-59-2$ & cis-1,2-Dichloroethene & 0.50 & U \\
\hline $78-93-3$ & 2-Butanone & 5.0 & 0 \\
\hline $74-97-5$ & Bromochloromethane & 0.50 & $\mathrm{U}$ \\
\hline $67-66-3$ & Chloroform & 0.50 & U] \\
\hline $71-55-6$ & 1,1,1-Trichloroethane & 0.50 & U \\
\hline $110-82-7$ & Cyclohexane & 0.50 & $\mathrm{U}$ \\
\hline $56-23-5$ & Carbon tetrachloride & 0.035 & $\mathrm{~J}$ \\
\hline $71-43-2$ & Benzene & 0.50 & U \\
\hline $107-06-2$ & 1,2-Dicinloroethane & 0.50 & $\mathrm{t}$ \\
\hline
\end{tabular}

Report 1,4-Dioxane for Low-Medium voA analysis only 
IE - EORM I VOA-2

VOLATILE ORGANICS ANAJYSIS DATA SHEET
EPE SAYPIE NO.

MRNW6S-W-30044

Lab Name: TESTAMERTCA BURLINGTON

Lab Code: STI.V

Case No.: MORRII, Mod, Ref No, :

Contract: $\quad 8 \mathrm{E}-00302$

Matrix: (SOIL/SED/WATER)

Sample wt/vol: 25.0 Water

(g/mIs) mis

Level: (TRACE/LOW/MED) TRACE

\% Moisture: not dec.

GC Column: DB-624 ID: 0.20 (nim)

Soil Extract volume:

Purge Volume: 25.0

ID: 0,20

(uJ.)

$(\mathrm{mL})$

SLG No.: 200-4827

Lab Sample ID: 200-4827-2

Lab File 1D: JCUHO5.D

Date Received: $04 / 22 / 2011$

Date Analyzed: $04 / 25 / 2011$

Dilution Factor: 1.0

Soil Aliquot Volume:

(บL)

\begin{tabular}{|c|c|c|c|}
\hline CAS NO. & COMPOUND & $\begin{array}{l}\text { CONCENIRAIION JNTTS: } \\
(\mathrm{ug} / \mathrm{L} \text { or } \mathrm{ug} / \mathrm{kg}) \mathrm{ug} / \mathrm{L}\end{array}$ & $Q$ \\
\hline $79-01-6$ & Trichloroethene & 0.50 & $\mathrm{U}$ \\
\hline $108-87-2$ & Wethylcyclohexane & 0.50 & $\mathrm{U}$ \\
\hline $78-87-5$ & 1,2-Dichloropropare & 0.50 & $\mathrm{C}$ \\
\hline $75-27-4$ & Bromodichloromethane & 0.50 & $\mathrm{U}$ \\
\hline $10061-01-5$ & cis-1,3-Dichloropropene & 0.50 & 0 \\
\hline $108-10-1$ & 4-Methyl-2-pentanone & 5.0 & 0 \\
\hline $108-88-3$ & Toluene & 0.019 & $\mathrm{~J} \mathrm{~B}$ \\
\hline $10061-02-6$ & trans-1,3-Dichloropropene & 0.50 & $\mathrm{U}$ \\
\hline $79-00-5$ & 1,1,2-Trichloroethane & 0.50 & U \\
\hline $127-18-4$ & Tetrachloroethene & 0.50 & $\mathrm{U}$ \\
\hline $591-78-6$ & 2-Hexanone & 5.0 & U \\
\hline $3.24-48-1$ & Dibromochloromethane & 0.50 & U \\
\hline $106-93-4$ & 1,2-Dibromoetharie & 0.50 & U \\
\hline $108-90-7$ & Chlorobenzene & 0.50 & J \\
\hline $100-41-4$ & Ethylbenzerse & 0.022 & $\mathrm{~J}$ \\
\hline $95-47-6$ & o-xylene & 0.50 & $U$ \\
\hline $179601-23-1$ & $m, p-x y l$ ene & 0.064 & J \\
\hline $1,00-42-5$ & styrene & 0.50 & U \\
\hline $75-25-2$ & Bromoform & 0.50 & $\mathrm{U}$ \\
\hline $98-82-8$ & Isopropylbenzene & 0.50 & ] \\
\hline $79-34-5$ & $1,1,2,2$-Tetrachloroetbane & 0.50 & 0 \\
\hline $541-73-1$ & 1,3-Dichlorobenzene & 0.50 & U \\
\hline $106-46-7$ & 1,4-Dichlorobenzene & 0.50 & $\mathrm{U}$ \\
\hline $35-50-1$ & 1,2 -Dichlorobenzene & 0.50 & $\mathrm{U}$ \\
\hline $96-12-8$ & 1,2-Dibrono-3-Chiloropropane & 0.50 & U \\
\hline $120-82-1$ & 1,2,4-Trichlorobenzene & 0.50 & $\mathrm{U}$ \\
\hline $87-61-6$ & 1,2,3-Trichlorobenzene & 0.50 & $\mathrm{U}$ \\
\hline
\end{tabular}


$1 \mathrm{~J}-$ EORM I VOA-TIC VOLATILE ORGANICS ANAI,YSIS DATA SHEET IENTATIVELY IDENTIFIED COMPOUNDS
EPA SAMPLE NO. MRMW 6S-W-30044

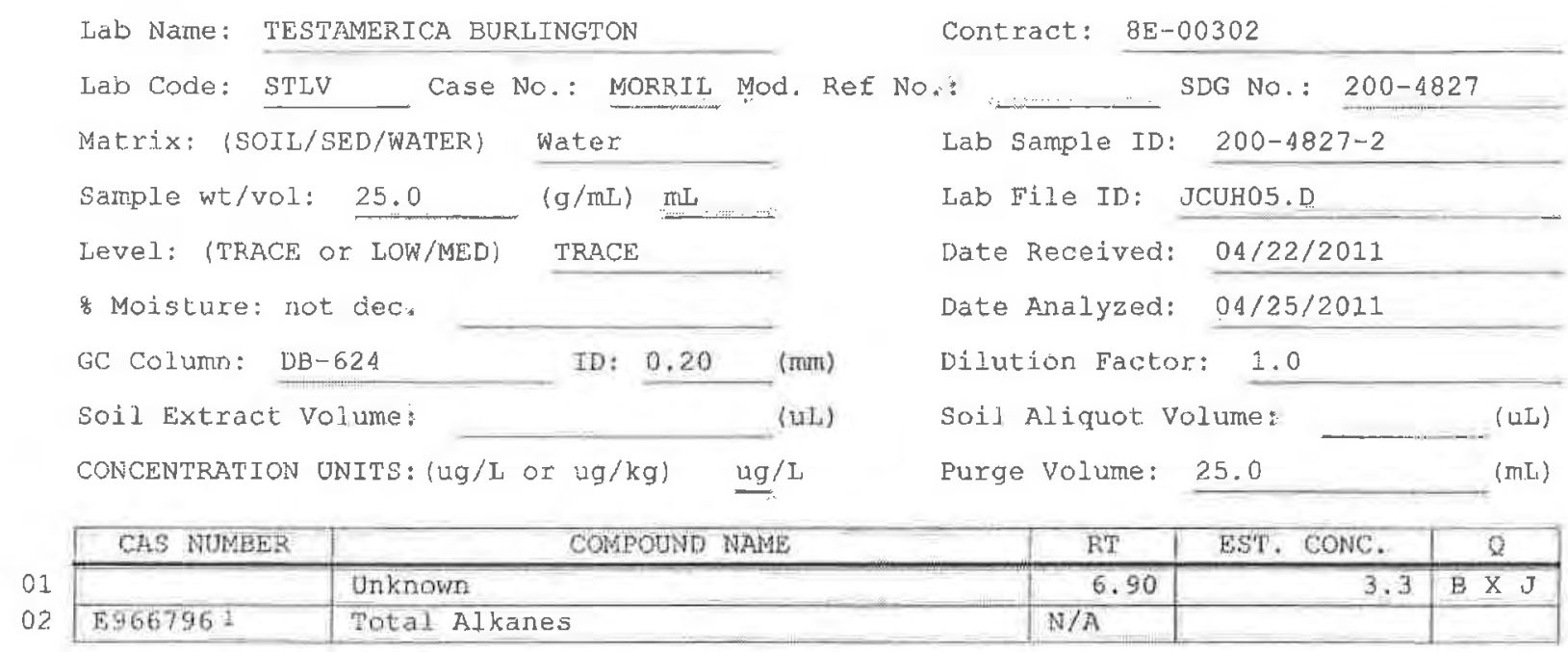

1 EPA-designated Registry Number. 
$1 A$ - FORM I VOA-1

VOLATILE ORGANICS ANALYSIS DATA SHEET
IPA SAMPLE NO.

MRQTTB-W-30061
Lab Name: TESTAMERICA BURLINGTON

Lab Code: STLV_... Case No.: MORRIL Mod, Ref No.:

Matrix: (SOII/SED/WATER) Water

Sample wt/vol: $25.0 \quad(\mathrm{~g} / \mathrm{mL}) \mathrm{mL}$

Level: (TRACE/LOW/MED) TRACE

g Moisture: not dec.

GC Colurn: DB-624 I.D: 0.20 (mun)

Soil Extract Volume:

Purge Volume: 25.0
Contract: $\quad 8 E-00302$

SDG NO.: $200-4827$

Lab Sample ID: 200-4827-5

Lab File ID: JCUH08.D

Date Received: 04/22/2011

Date Analyzed: 04/25/2011

Dilution Factor: 1.0

Soi.l A.liquot Volume: (uL)

\begin{tabular}{|c|c|c|c|}
\hline CAS NO. & COMPOUND & $\begin{array}{l}\text { CONCENTRATTON UNITS: } \\
\{\mathrm{ug} / \mathrm{L} \text { or } \mathrm{ug} / \mathrm{kg}\rangle \mathrm{ug} / \mathrm{L}\end{array}$ & $Q$ \\
\hline $75-71-8$ & Dichlorodifluoromethane & 0.50 & U \\
\hline $74-87-3$ & Chloromethane & 0.50 & $\mathrm{U}$ \\
\hline $75-01-4$ & Vinyl chloride & 0.50 & U \\
\hline $74-83-9$ & Bromomethane & 0.50 & 0 \\
\hline $75-00-3$ & Chloroethane & 0.50 & v \\
\hline $75-69-4$ & Trichlorofluoromethane & 0.50 & $\mathrm{U}$ \\
\hline $75-35-4$ & 1,1-Dichlorsethene & 0.50 & U \\
\hline $76-13-1$ & 1,1,2-Trichloro-1,2,2-trifluoroethane & 0.50 & $\mathrm{U}$ \\
\hline $67-64-1$ & Acetone & 4.0 & $\mathrm{~J} \mathrm{~B}$ \\
\hline $75-15-0$ & Cärbon disulfide & 0.083 & $\mathrm{JB}$ \\
\hline $79-20-9$ & Methyl acetate & 0.50 & $\mathrm{U}$ \\
\hline $75-09-2$ & Methylene Chloride & 0.50 & U \\
\hline $156-60-5$ & trans-1,2-Dichloroethene & 0.50 & $\mathrm{U}$ \\
\hline $1634-04-4$ & Methyl tert-butyl ether & 0.50 & $\mathrm{U}$ \\
\hline $75-34-3$ & 1, 1-Dichloroethane & 0.50 & U \\
\hline $156-59-2$ & cis-1,2-Dichloroethene & 0.50 & U \\
\hline $78-93-3$ & 2-Butanone & 5.0 & $\mathrm{U}$ \\
\hline $74-97-5$ & Bromochloromethane & 0.50 & 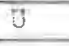 \\
\hline $67-66-3$ & Chloroform & 0.50 & $\mathrm{U}$ \\
\hline $71-55-6$ & $1,1,1$-Trichloroethane & 0.50 & $\mathrm{j}$ \\
\hline $110-82-7$ & Cyclohexane & 0.50 & U \\
\hline $56-23-5$ & Carbon tetrachioride & 0.023 & $\mathrm{~J}$ \\
\hline $71-43-2$ & Benzene & 0.025 & $J$ \\
\hline $107-06-2$ & 1,2-Dichloroethane & 0.50 & U \\
\hline
\end{tabular}

Report 1,4-Dioxane for Low-Medium VOR analysis only 
IB - FORM I VOA-2

VOTATILE ORGANICS ANALYSTS DATA SHEE'T
EPA SAMPLE NO.

MROCTB-W- 3006

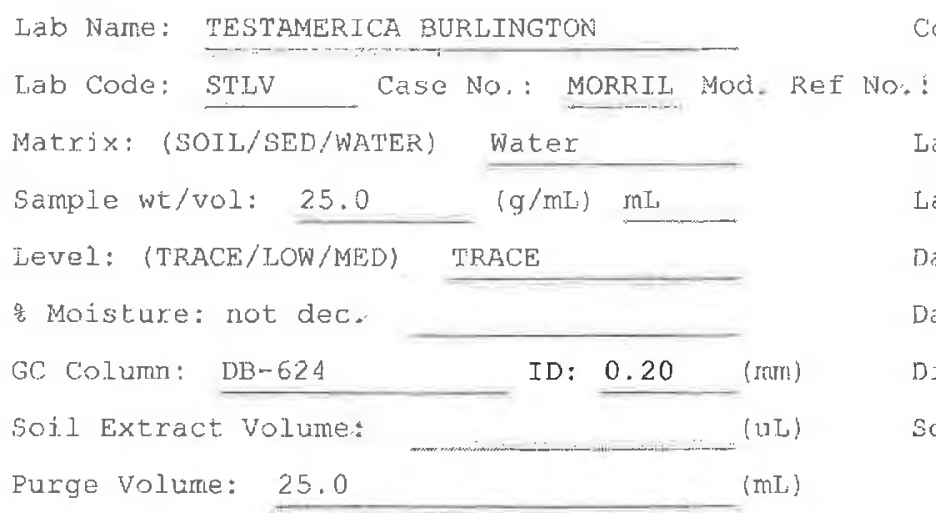
Purge Volume: 25.0 (mL)

\begin{tabular}{|c|c|c|c|}
\hline CAS NO. & COMPOUND & $\begin{array}{l}\text { CONCENT:RATION UNITS: } \\
(\mathrm{ug} / \mathrm{I} \text { or } \mathrm{ug} / \mathrm{kg}) \mathrm{Lg} / \mathrm{L}\end{array}$ & Q \\
\hline $79-01-6$ & Trichlorocthene & 0.50 & $\mathrm{U}$ \\
\hline $108-97-2$ & Methylcyclohexane & 0.50 & U \\
\hline $78-87-5$ & 1,2-Dichloropropane & 0.50 & $\mathrm{U}$ \\
\hline $75-27-4$ & Bromodichloromethane & 0.50 & U \\
\hline $10061-01-5$ & cis-1,3-Dichloropropene & 0.50 & $\mathrm{U}$ \\
\hline $108-10-1$ & 4-Methyl-2-pentarione & 5.0 & $\mathrm{U}$ \\
\hline $1083-88-3$ & Toluene & 0.51 & B \\
\hline $10061-02-6$ & trans-1,3-Dichloropropene & 0.50 & ए \\
\hline $79-00-5$ & $1,1,2$-Trichloroethane & 0.50 & U \\
\hline $127-.18-4$ & Tetrachloroethene & 0.50 & $\mathrm{U}$ \\
\hline $59 l-78-6$ & 2-Hexanone & 5.0 & U \\
\hline $124-48-1$ & Dibromochloromethane & 0.50 & U \\
\hline $106-93-4$ & 1,2-Dibromoethane & 0.50 & 0 \\
\hline $108-90-7$ & Chlorobenzene & 0.50 & U \\
\hline $100-41-4$ & Ethylbenzene & 0.055 & I \\
\hline $95-47-6$ & $0-X y l e n e$ & $0.1 \bar{I}$ & J \\
\hline $179601-23-1$ & $m, p-x y l e n e$ & 0.22 & I \\
\hline $100-42-5$ & Styrene & 5.50 & u \\
\hline $75-2.5-2$ & Byomoform & 0.50 & U \\
\hline $38-82-8$ & Isopropylbenzene & 0.50 & U \\
\hline $79-34-5$ & $1,1,2,2$-Tetrachloroethane & 0.50 & U \\
\hline $541-73-1$ & 1,3-Dichlorobenzene & 0.50 & $\mathrm{U}$ \\
\hline $106-46-7$ & 1, 4-Dichlorobenzene & 0.50 & U \\
\hline $55-50-1$ & 1,2-Dichlorobenzene & 0.50 & $\mathrm{U}$ \\
\hline $96-12-3$ & 1,2-Dibromo-3-Chlogopropane & 0.50 & $\mathrm{U}$ \\
\hline $120-82-1$ & 1,2, 4-Trichlorbbenzene & 0.50 & 0 \\
\hline $87-61-6$ & $3,2,3$-Trjohlorobenzene & 0.50 & $\mathrm{U}$ \\
\hline
\end{tabular}


$1 \mathrm{~J}$ - FORM I VOA-TIC

VOLATILE ORGANICS ANALYSIS DATA SHEET' TENTATIVELY IDENTIFIED COMPOUNDS
EPA SAMELE NO.

MRQCTB-W-30061
Lab Name: TESTMMERICA BURLINGTON

Lab Code: STLV Case No.: MORRIL Mod. Ref No.

Contract: $\quad 8 \mathrm{E}-00302$

Matrix: (SOII/SED/AATER)

Sample wt/vol: 25.0

Water

$(\mathrm{g} / \mathrm{mL}) \mathrm{mL}$

Level: (TRACE or LOW/MED)

TRACE

o Moisture: not dec.

GC Column: DB-624

Soil Extract Volume: ID: $0.20 \quad(\mathrm{~mm})$

CONCENTRATION UNITS: (ug/L or $u g / \mathrm{kg}$ ) ug/L (u, IJ)
SDG No : $\quad 200 \cdots 4827$

Lab Sample ID: 200-4827-5

Lab rile I.D: JCUH08.D

Date Recefved: $04 / 22 / 2011$

Date Analyzed: $04 / 25 / 2011$

Dilution Eactor: 1.0

Soil Aliquot Volume:

Purge Volume: 25.0 $\left(m \bar{i}_{1}\right)$

\begin{tabular}{|c|c|c|c|c|}
\hline CAS NUMBER & COMPOUND NAME & RT & EST. CONC. & Q \\
\hline & Unknown & 6.90 & 3.0 & $B \times J$ \\
\hline E9667961 & Total Alkanes & $\mathrm{N} / \mathrm{A}$ & 40 & $\mathrm{~J}$ \\
\hline
\end{tabular}

\footnotetext{
1 ERA-designated Registry Number.
} 
IA - FORM I VOA-1

VOLATTLE ORGANICS ANALYSIS DATA SHEET
EPA SAMPLE NO

MRRILLINGER-W- 300
I.ab Name: TESTAMERICA BURLINGTON

Lab code: STLV Case No.: MORRIL Mod. Ref No.:
Water

$(\mathrm{g} / \mathrm{mL}) \quad \pi \mathrm{N}$

Sample wt/vol: 25.0

LeVe 1: (TRACE/LOW/MED) TRACE

* Mojstuxe: hot dec.

GC CoIumn: DB-624

Soil Extract Volume:

Purge Volume: 25.0
Contract: $\quad$ BE-00302

SDE NO.: 200-4827

Lab sample ID: $\quad 200-4827-4$

Lab File ID; JCUH07.D

Date Received: $04 / 22 / 2011$

Date Analyzed: $04 / 25 / 2011$

Dilution Factor: 1.0

Soll Aliquot volume: (mL)

\begin{tabular}{|c|c|c|c|}
\hline CAS NO. & COMPOUND & $\begin{array}{l}\text { CONCENTRATION UNISS: } \\
(\mathrm{ug} / \mathrm{L} \text { or } \mathrm{ug} / \mathrm{kg}) \mathrm{Lg} / \mathrm{L}\end{array}$ & $Q$ \\
\hline $75-71-8$ & Dichlorodifluoromethane & 0.50 & $\overline{\mathrm{U}}$ \\
\hline $74-87-3$ & Chloromethane & 0.50 & $\mathrm{U}$ \\
\hline $75-0]-4$ & Vinyl chloride & 0.50 & $\mathrm{t}$ \\
\hline $74-83-9$ & Bromomethane & 0.50 & [I \\
\hline $75-00-3$ & Chloroethane & 0.50 & U \\
\hline $75-69-4$ & Trichlorofluoromethane & 0.50 & U \\
\hline $75-35-4$ & 1,1-Dichloroethere & 0.50 & $\mathrm{U}$ \\
\hline $76-13-1$ & 1,1,2-Trichloro-1,2,2-trifluoroethane & 0.50 & $\mathrm{U}$ \\
\hline $67-54-1$ & Acetone & 1.2 & J B \\
\hline $75-15-0$ & Carbon disulfide & 0.078 & $\mathrm{JB}$ \\
\hline $79-20-9$ & Methyl acetate & 0.50 & $\mathrm{U}$ \\
\hline $75-09-2$ & Methylene Chloride & 0.50 & !) \\
\hline $156-60-5$ & trans-1,2-Dichloroethene & 0.50 & $\mathrm{U}$ \\
\hline $1634-04-4$ & Methyl tert-butyl ether & 0.50 & U \\
\hline $75-34-3$ & 1,1-Dichloroethane & 0.50 & $\mathrm{U}$ \\
\hline $156-59-2$ & cis-1, 2-Dichloroethere & 0.50 & $\mathrm{U}$ \\
\hline $78-93-3$ & 2-Butanone & 5.0 & U \\
\hline $74-97-5$ & Bromochloromethare & 0.50 & E \\
\hline $67-66-3$ & Chlaroforta & 0.50 & $\mathrm{U}$ \\
\hline $71-55-6$ & $1,1,1$-Trichloroethane & 0.50 & U \\
\hline $110-82-7$ & Cyclohexarie & 0.50 & $\mathrm{U}$ \\
\hline $56-23-5$ & Carbon tetrachloride & 0.39 & J \\
\hline $71-43-2$ & Benzene & 0.50 & U \\
\hline $107-06-2$ & 1,2-DichIoroethane & 0.50 & U \\
\hline
\end{tabular}


$1 B$ - FORM I VOA-2

VOIARIIL ORGANICS ANALYSIS DATA SHEET
EPA SAMPLE NO.

MFRI.TLINGER-W- 300
Lab Name:' TESTAMERICA BURLINGTON

Lab code: STLV

Case
Matrix: (SOIL/SED/WATER)

Sample wt/vol: 25.0

LeVel: (TRACE/IOW/MED)

soisture: not dec,

GC Column: DB-624

Soil Extract Volume:

Purge Volume: 25.0
Contract: $8 \mathrm{E}-00302$

SDG No.: 200-4827

Lab Sample ID: 200-4827-4

Lab File ID: JCUH0?.D

Date Received: 04/22/2011

Date Aralyzed: 04/25/2011

Dilution Factor: 1.0

Soid Aliquot volume:

(UL)

(uL) $(\pi \mathrm{L}$

\begin{tabular}{|c|c|c|c|}
\hline CAS NO. & COMPOUND & $\begin{array}{l}\text { CONCENTRATION UNITS: } \\
(\mathrm{ug} / \mathrm{L} \text { or } \mathrm{ug} / \mathrm{kg}) \mathrm{ug} / \mathrm{L}\end{array}$ & $Q$ \\
\hline $79-01-6$ & Trichloroethene & 0.50 & $\mathrm{U}$ \\
\hline $108-87-2$ & Methylcyclohexane & 0.50 & $\mathrm{U}$ \\
\hline $78-87-5$ & 1,2-Dichloropropane & 0.50 & U \\
\hline $75-27-4$ & Bromadichloromethane & 0.50 & $\mathrm{U}$ \\
\hline $10061-0.1-5$ & cis-1,3-Dichloropropere & 0.50 & U \\
\hline $108-10-1$ & 4-Methyl-2-pentanone & 5.0 & U \\
\hline $108-88-3$ & Toluene & 0.50 & $\mathrm{U}$ \\
\hline $10061-02-6$ & trans-1,3-Dichloropropene & 0.50 & $\mathrm{U}$ \\
\hline $79-00-5$ & 1,1,2-Trichloroethane & 0.50 & U \\
\hline $127-18-4$ & Tetrachloroethene & 0.50 & $\mathrm{U}$ \\
\hline $591-73-6$ & 2-Hexanone & 5.0 & D \\
\hline $124-48-1$ & Dibromochloromethane & 0.50 & $\mathrm{U}$ \\
\hline $106-93-4$ & 1,2-Dibromoethane & 0.50 & 0 \\
\hline $108-90-7$ & Chlorobenzene & 0.50 & U \\
\hline $100-41-4$ & Ethylbenzene & 0.50 & 0 \\
\hline $95-47-6$ & o-Xylene & 0.50 & 0 \\
\hline $179601-23-1$ & In, $\mathrm{P}-\mathrm{Xy}$ lene & 0.036 & J \\
\hline $100-42-5$ & Styrene & 0.50 & U \\
\hline $75-25-2$ & Bromoform & 0.50 & U \\
\hline $98-82-8$ & Isopropy Lbenzene & 0.50 & $\mathrm{U}$ \\
\hline $79-34-5$ & $1,1,2,2$-Tetrachloroethane & 0.50 & $\mathrm{U}$ \\
\hline $541-73-1$ & 1,3-Dichlorobenzene & 0.50 & $\mathrm{U}$ \\
\hline $105-46-7$ & 1,4-Dichlorobenzene & 0.50 & $\mathrm{U}$ \\
\hline $95-50-1$ & 1,2-Dichlorobenzene & 0.50 & $\mathrm{U}$ \\
\hline $96-12-8$ & 1,2-Dibromo-3-Chloropropane & 0.50 & $\mathrm{U}$ \\
\hline $120-82-1$ & 1,2,4-Trichiorobenzene & 0.50 & U \\
\hline $87-6.1-6$ & $1, \overline{2}, 3-5$ fichlorobenzene & 0.50 & 0 \\
\hline
\end{tabular}




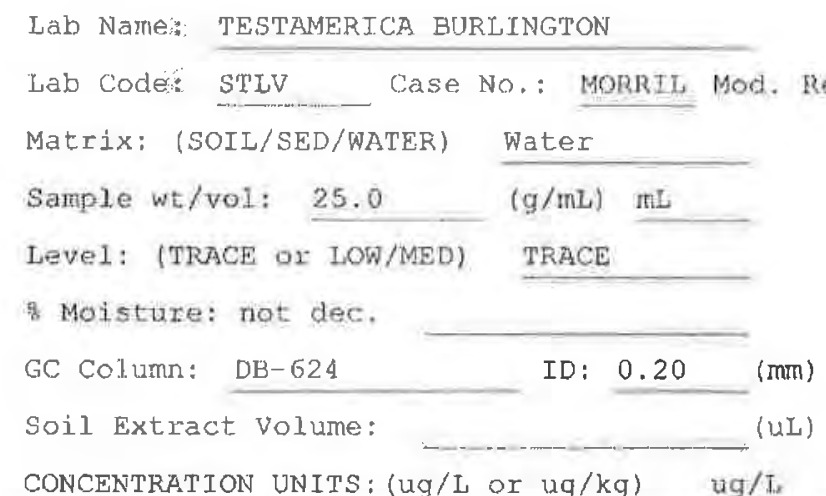

CONCENTRATION UNITS: (ug/L or $u g / \mathrm{kg}$ ) $\mathrm{ug} / \mathrm{I}$.
Contract: $8 E-00302$ SDG No.: $\quad 200-4827$

\begin{tabular}{|l|l|l|r|r|r|}
\hline \multicolumn{1}{|c|}{ CAS NUMBER } & \multicolumn{1}{|c|}{ COMPOUND NAME } & RT. & EST. CONC. & Q \\
\hline
\end{tabular}

\footnotetext{
1 EPA-designated Registry Number,
}

Lab Sample ID: 200-4827-4

Lab File ID: JCUH07.D

Date Received: 04/22/2011

Date Analyzed: 04/25/2011

Dilution Factor: 1.0

Soil Aliquot volume: (uL)

Purge volume: 25.0 (mL)
01

(2) 
6A -- EORM VI VOA-1

VOLATILE ORGANICS INTTIAL CALIBRATION DATA

\begin{tabular}{|c|c|c|c|c|c|c|c|}
\hline Jab Name: TESTAMERICA BURLIN & CON & & Contract & $8 \mathrm{E}-1$ & & & \\
\hline Lab Code: STLV & MORRIL Mod & d. Ref No & & SDC & G No.: 2 & $00-4827$ & \\
\hline Instrumert ID: $\mathrm{J} . \mathrm{i}$ & & CaIibrat & ion Date & (s): & $/ 24 / 2011$ & $03 / 21 /$ & 2011 \\
\hline Heated Purge: (Y/N) & & Calibrat & ion Time & (s): : & & 1549 & \\
\hline Purge volume: 25.0 & & $(\mathrm{mi})$ & & & & & \\
\hline GC Column: $\mathrm{DB}-624$ & ID: 0.20 & $(\mathrm{~mm})$ & Length: & 25 & $(\mathrm{~m})$ & & \\
\hline LAB FILE' ID: & RRF0.5 $=\pi$ & 003.1 & & RRF 1. & $0=J \mathrm{CUO}$ & & \\
\hline RRF $5.0=\mathrm{JCUO5.D}$ & $\mathrm{RRF} 10=\mathrm{J} S$ & $.006 . \mathrm{D}$ & & RRF 20 & $=\mathrm{JCUO}$ & $7 . \mathrm{D}$ & \\
\hline COMPOUND & RRE 0.5 & RRE 1.0 & RRE 5.0 & RRF 10 & RRE 20 & $\overline{R R E}$ & \&RSD \\
\hline Dichlorodifluoromethane & 0.544 & 0.601 & 0.553 & 0.532 & 0.488 & 0.544 & 7.4 \\
\hline Chloromethane & 0.438 & 0.442 & 0.422 & 0.410 & 0.366 & 0.416 & 7.4 \\
\hline Vinyl chloride & 0.429 & 0.440 & 0.423 & 0.405 & 0.362 & 0.412 & 7.5 \\
\hline Bromiomethane & 0.233 & 0.236 & 0.233 & 0.204 & 0.180 & 0.217 & 11.2 \\
\hline Chloroethane & 0.222 & 0.244 & 0.222 & 0.213 & 0.190 & 0.218 & 8.9 \\
\hline Trichjorofiuoronethane & 0.544 & 0.635 & 0.621 & 0.602 & 0.549 & 0.610 & 6.2 \\
\hline 1,2-Dichloroethere & 0.286 & 0.304 & 0.308 & 0.289 & 0.255 & 0.288 & 7.3 \\
\hline $\begin{array}{l}\text { 1,1,2-Trichloro- } \\
1,2,2 \text {-trifluoroethane }\end{array}$ & 0.345 & 0.374 & 0.349 & 0.334 & 0.301 & 0.341 & 7.8 \\
\hline Acetone & 0.018 & 0.015 & 0.015 & 0.016 & 0.014 & 0.016 & 10.8 \\
\hline Carbon disulfide & 0.991 & 0.897 & 0.906 & 0.850 & 0.813 & 0.891 & 7.5 \\
\hline Methyi acetate & 0.041 & 0.052 & 0.045 & 0.046 & 0.044 & 0.046 & 9.0 \\
\hline Methyiene ChIoride & 0.277 & 0.265 & 0.272 & 0.261 & 0.246 & 0.264 & 4.5 \\
\hline trans-1,2-Dichloroethene & 0.336 & 0.336 & 0.349 & 0,338 & 0.315 & $0 . \overline{335}$ & 3.7 \\
\hline Methyl tert-butyl ether & 0.361 & 0.385 & 0.390 & 0.394 & 0.382 & 0.382 & 3.3 \\
\hline 1,1-Dichioroethane & 0.577 & 0.568 & 0.566 & 0.558 & 0.520 & 0.558 & 3.9 \\
\hline cis-1,2-Dichloroethene & 0.306 & 0.331 & 0.326 & 0.325 & 0.307 & 0.319 & 3.6 \\
\hline 2-Butarione & 0.020 & 0.025 & 0.026 & 0.026 & 0.026 & 0.024 & 11.2 \\
\hline Bromochloromethane & 0.107 & 0.109 & 0.108 & 0.101 & 0.101 & 0.106 & $2 . \overline{8}$ \\
\hline Chiloreform & 0.528 & 0.560 & 0.559 & 0.537 & 0.510 & 0.539 & 4.0 \\
\hline 1,1,1-Trichioroethane & 0.679 & 0.694 & 0.719 & 0.682 & $0.67 \mathrm{~A}$ & 0.689 & 2.6 \\
\hline Cyclohexane & 0.603 & 0.630 & 0.720 & 0.698 & 0.699 & 0.670 & 7.5 \\
\hline Carbon tetrach oride & 0.610 & 0.622 & 0.658 & 0.634 & 0.530 & 0.631 & 2.8 \\
\hline Benzene & 1.466 & 1.610 & 1.649 & 1.624 & 1.611 & 1.592 & 4.5 \\
\hline 1.,2-Dichloloethane & 0.210 & 0.221 & 0.217 & 0.226 & 0.208 & 0.216 & 3.7 \\
\hline Trichloroethene & 0.381 & 0.414 & 0.421 & 0.408 & 0.410 & 0.407 & 3.4 \\
\hline Methylcyclonexane & 0.464 & 0.484 & 0.516 & 0.519 & 0.515 & 0.500 & 4.9 \\
\hline
\end{tabular}

Report 1,4-Dioxane for Low-Medium VoA analysis only 
$6 B$ - FORM VI VOA-2

VOLATILE ORGANICS INITIAL CALIBRATION DATA

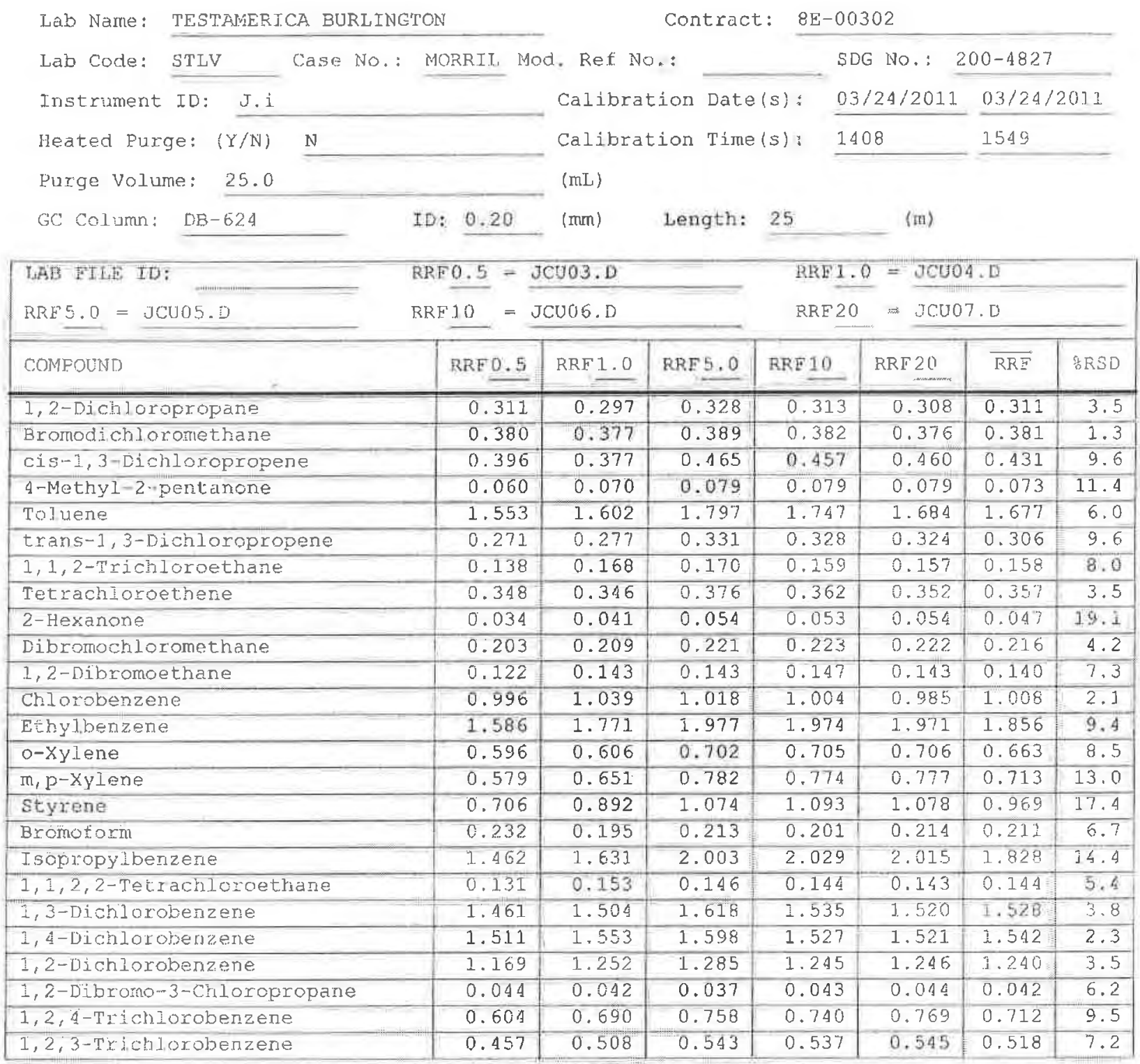


$6 \mathrm{C}$ - FORM VI VOA-3

VOLATILE ORGANICS INITIAL CALIBRATION DATA

\begin{tabular}{|c|c|c|c|c|c|c|c|}
\hline Láb Name: TESTAMERICA BURLING & TON & & Contract & $8 E-00$ & 02 & & \\
\hline Lab code: STLV & MORRIL MOd & 1. Bef No & & $S D G$ & No.: & $00-4827$ & \\
\hline Instrument ID: $\mathrm{J} . i$ & & Calibrat & ion Date & $(s):$ & $24 / 2011$ & $03 / 24 /$ & 2011 \\
\hline Heated Purge: $(\mathrm{Y} / \mathrm{N})$ & & Calibrat & ion Time & $\{s\}:$ & & 1549 & \\
\hline Purge Volume: 25.0 & & $(m L)$ & & & & & \\
\hline GC Colurm: $\quad$ DB-624 & ID: 0.20 & $(\mathrm{~mm})$ & Length: & 25 & (m) & & \\
\hline LAB FILE ID; & RRFO. $0.5=J$ & CU03.D & & RRE 1. & $=\pi \mathrm{JCU} 0$ & 1.D & \\
\hline RRF $5.0=J C U 05 . D$ & $\operatorname{RRE} 10=\mathrm{J}$ & $006 . \mathrm{D}$ & & RRF 20 & $\Rightarrow \mathrm{JCU} 0$ & $\cdot \mathrm{D}$ & \\
\hline COMPOUND & RRE 0.5 & RRF 1.0 & RRF 5.0 & RRF 10 & $\operatorname{RRF} 20$ & $\overline{\mathrm{RRE}}$ & ERSD \\
\hline Vinyl Chloride-d3 & 0.390 & 0.401 & 0.373 & 0.356 & 0.320 & 0.368 & 8.7 \\
\hline Chiloroethane-d5 & 0.310 & 0.310 & 0.285 & 0.263 & 0.228 & 0.279 & 12.5 \\
\hline 1,1-Dichloröthene-d2 & 0.600 & 0.671 & 0.636 & 0.611 & 0.555 & 0.615 & 7.0 \\
\hline 2-Butanone-d5 & 0.025 & 0.025 & 0.026 & 0.027 & 0.026 & 0.026 & 4.1 \\
\hline Chloroform-d & 0.587 & 0.591 & 0.583 & 0.565 & 0.535 & 0.572 & 4.0 \\
\hline 1,2-Dichloroethane-d 4 & 0.183 & 0.182 & 0.183 & 0.182 & 0.172 & 0.180 & 2.7 \\
\hline Benzene-do & 1.411 & 1.574 & 1.642 & 1.61 .3 & 1.586 & 1.565 & 5.8 \\
\hline 1,2-Dichloropropane-d6 & 0.419 & 0.358 & 0.427 & 0.419 & 0.359 & 0.396 & 8.8 \\
\hline Toluene-d8 & 1.263 & 1.374 & 1.546 & 1.505 & 1.445 & 1.427 & 7.8 \\
\hline trans-1,3-Dichloropropene-d4 & 0.238 & 0.255 & 0.297 & 0.300 & 0.296 & 0.277 & 10.3 \\
\hline 2-Hexanone-d5 & 0.018 & 0.023 & 0.029 & 0.030 & 0.030 & 0.026 & 20.3 \\
\hline $1,1,2,2$-Tet rachloroethane-d2 & 0.140 & 0.151 & 0.154 & 0.151 & 0.147 & 0.149 & 3.7 \\
\hline 1, 2-Dichlorobenzene-d4 & 0.772 & 0.792 & 0.822 & 0.775 & 0.779 & 0.788 & 2.6 \\
\hline
\end{tabular}

Report 1,4-Dioxane-d8 for Low-Medium VoA analysis only 
7A - FORY VII VOA-1

VOLATILE CONIINUING CALLBRATION DATA

Iab Name: TESTAMTRICA BURLINGTON

Contract: $8 E-00302$

lab Code: STLu' Case No, MORRII, Mod. Ref No.

SDG NO.: $200-4827$

Instrument: ID: J.i

Calibration Date: 04/25/2011 Tine: 0948

Lab File Id: JCUH02.D

EPA. Sample No. (VSTD聿\#\#)： VSTD005.jZ Init. Calib. Date(s): 03/24/2011 03/24/2011

Feated purge: ( $Y / N$ ) $N$ GC Colum:

Init. Calib. Time(s): $1408 \quad 1549$

purge volume: 25.0

ID: 0.20 (mrn) Length: 25

(m)

\begin{tabular}{|c|c|c|c|c|c|}
\hline CONPOUND & $\overline{\mathrm{RRF}}$ & RRE5.0 & $\begin{array}{l}\text { MIN } \\
\text { RRE }\end{array}$ & s. & WnX $\approx 0$ \\
\hline Dichlorodifluoromethare & 0.544 & 0.479 & 0.010 & $-12 \cdot 0$ & 40.0 \\
\hline Chloromethane & 0.416 & 0.327 & 0.010 & -21.4 & 40.0 \\
\hline Viny1 chloride & 0.412 & 0.349 & 0.010 & -15.3 & 30.0 \\
\hline Bromometrane & 0.217 & 0.192 & 0.100 & -11.8 & 30.0 \\
\hline Chloroetharie & 0.218 & 0.192 & 0.010 & -12.0 & 40.0 \\
\hline Trichlorofluoromethane & 0.610 & 0.598 & 0.010 & -2.0 & 40.0 \\
\hline 1,1-Dichloroethene & 0.288 & 0.291 & 0.100 & 1.2 & 30.0 \\
\hline 1, 1,2-Trichloro-1,2,2-trifluoroethane & 0.341 & 0.340 & 0.010 & -0.2 & 40.0 \\
\hline Acetone & 0.016 & 0.016 & 0.010 & 0.1 & 40.0 \\
\hline Carbon disulfide & 0.891 & 0.913 & 0.010 & 2.5 & 40.0 \\
\hline Methyl acetate & 0.046 & 0.046 & 0.010 & 0.7 & 40.0 \\
\hline Methylene Chloride & 0.264 & 0.274 & 0.010 & 3.6 & 40.0 \\
\hline trans $-1,2-$ Dichloroethene & 0.335 & 0.361 & 0.010 & 7.7 & 40.0 \\
\hline Methyl tert-butyl ether & 0.382 & 0.411 & 0.010 & 7.6 & 40.0 \\
\hline 1,1-Dichloroethane & 0.558 & 0.556 & 0.200 & -0.4 & 30.0 \\
\hline cis-1,2-Dichloroethene & 0.319 & 0.347 & 0.010 & 8.9 & 40.0 \\
\hline 2-Butanone & 0.024 & 0.025 & 0.010 & 4.2 & 40.0 \\
\hline Bromochloromethare & 0.106 & 0.121 & 0.050 & 14.8 & 30.0 \\
\hline Chloroform & 0.539 & 0.567 & 0.200 & 5.2 & 30.0 \\
\hline 1,1,1-Trich Joroethane & 0.689 & 0.753 & 0.100 & 9.2 & 30.0 \\
\hline Cyclohexane & 0.670 & 0.702 & 0.010 & 4.8 & 10.0 \\
\hline Carbon tetrachloride & 0.631 & 0.724 & 0.100 & 14.8 & 30.0 \\
\hline Benzere & 1.592 & 1.677 & 0.400 & 5.3 & 30.0 \\
\hline 1,2-Dichloroethane & 0.216 & 0.237 & 0.100 & 9.3 & 30.0 \\
\hline Trichloroethene & 0.407 & 0.455 & 0.300 & 11.6 & 30.0 \\
\hline Methylcyclohexane & 0.500 & 0.526 & 0.010 & 5.4 & 40.0 \\
\hline
\end{tabular}

Report 1,4-Diorane for Low/Medium VOA analysis only 
$7 B$ - FORM VII VOA -2

VOLA'TIIJE CONTINUING CALIBRATION DATA

Lab Name: TESTAMERICA BURLINGTON

Contract: $8 \mathrm{E}-00302$

Lab code: STLV Case No.: MORRIL Mod. Ref ño.:

SDG NO.: 200-4827

Instrument ID: J.i

Calibration Date: 04/25/2011 Time: 0948

Lab File Id: JCuH02, D

EPA Sample No, (VSTD\#\#\#): VSTD005Jz Init. Calib. Date(s): 03/24/2011 03/24/2011

heated Purge: $(\mathrm{Y} / \mathrm{N})$

GC Column: $\mathrm{DB}-624$

Init. Calib. Time $\{s\}: 1408 \quad 1549$

purge volume: 25.0 ID: $0.20(\mathrm{~mm})$ Length: 25 (m)

(mL)

\begin{tabular}{|c|c|c|c|c|c|}
\hline COMPOUND & $\overline{\mathrm{RRE}}$ & RRF5.0 & $\begin{array}{l}\text { MIN } \\
\text { RRE }\end{array}$ & $81)$ & MaX of D \\
\hline 1,2-Lichloropropane & 0.311 & 0.325 & 0.010 & 4.3 & 40.0 \\
\hline Bromodichloromethane & 0.381 & 0.420 & 0.200 & 10.4 & 30.0 \\
\hline cis-1,3-Dichloropropene & 0.431 & 0.496 & 0.200 & 15.1 & 30.0 \\
\hline 4-Methyl-2-pentanone & 0.073 & 0.077 & 0.010 & 5.5 & 40.0 \\
\hline Toluene & 1.677 & 1.885 & 0.400 & 12.4 & 30.0 \\
\hline trans--1,3-Dichioropropene & 0.306 & 0.349 & 0.100 & 14.1 & 30.0 \\
\hline $1,1,2$-Trichloroethane & 0.158 & 0.178 & 0.100 & 12.8 & 30.0 \\
\hline Tetrachioroethene & 0.357 & 0.418 & 0.200 & 17.1 & 30.0 \\
\hline 2-Rexanane & 0.047 & 0.051 & 0.010 & 8.7 & 40.0 \\
\hline Dibromoch lor onethane & 0.216 & 0.250 & 0.100 & 16.0 & 30.0 \\
\hline 1,2-Dibromoethane & 0.140 & 0.164 & 0.010 & 17.6 & 40.0 \\
\hline Chlorobenzene & 1.008 & 1.119 & 0.500 & 10.9 & 30.0 \\
\hline Ethylbenzene & 1.856 & 2.076 & 0.100 & 11.9 & 30.0 \\
\hline o-xylene & $0.66 \overline{3}$ & 0.763 & 0.300 & 15.1 & 30.0 \\
\hline m,p-Xylene & 0.713 & 0.826 & 0.300 & 15.9 & 30.0 \\
\hline Styrene & 0.969 & 1.156 & 0.300 & 19.4 & 30.0 \\
\hline Bromoform & 0.211 & 0.246 & 0.050 & 16.6 & 30.0 \\
\hline Isopropylbenzere & 1.828 & 2.153 & 0.010 & 17.8 & 40.0 \\
\hline $1,1,2,2$-Tetrachloroethane & 0.144 & 0.156 & 0.100 & 8.6 & 30.0 \\
\hline 1,3-Dichloroberizene & 1.528 & 1.791 & 0.400 & 17.2 & 30.0 \\
\hline 1,4-Dichlorobenzene & 1.542 & 1.747 & 0.400 & 13.3 & 30.0 \\
\hline 1,2-Dichlorobenzene & 1.240 & 1.405 & 0.400 & 13.4 & 30.0 \\
\hline 1,2-Dibrorno-3-Ch loropsopane & 0.042 & 0.044 & 0.010 & 5.8 & 40.0 \\
\hline $1,2,4-\operatorname{Tr} i c h$ lorobenzene & 0.712 & 0.830 & 0.200 & 16.5 & 30.0 \\
\hline $1,2,3$-Trichlorobenzene & 0.518 & 0.560 & 0.200 & 8.1 & 30.0 \\
\hline
\end{tabular}


$7 \mathrm{C}$ - FORM VII VOA-3

VOLATELE: CONTINUTNG CALIBRATION DAPA

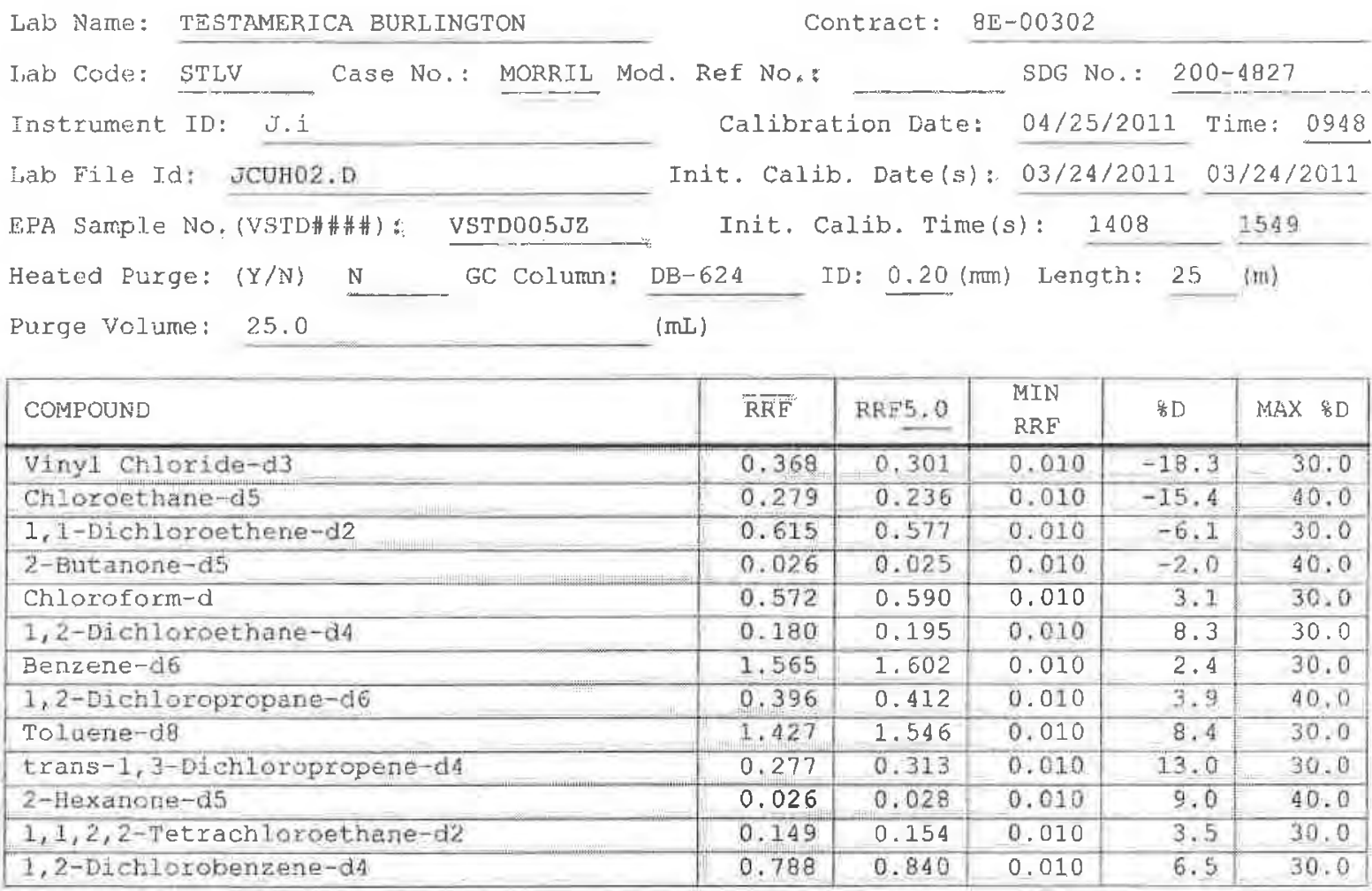


$7 A$ - FORM VII VOA-1

VOIATITE CONTINUING CAIIBRATION DATA

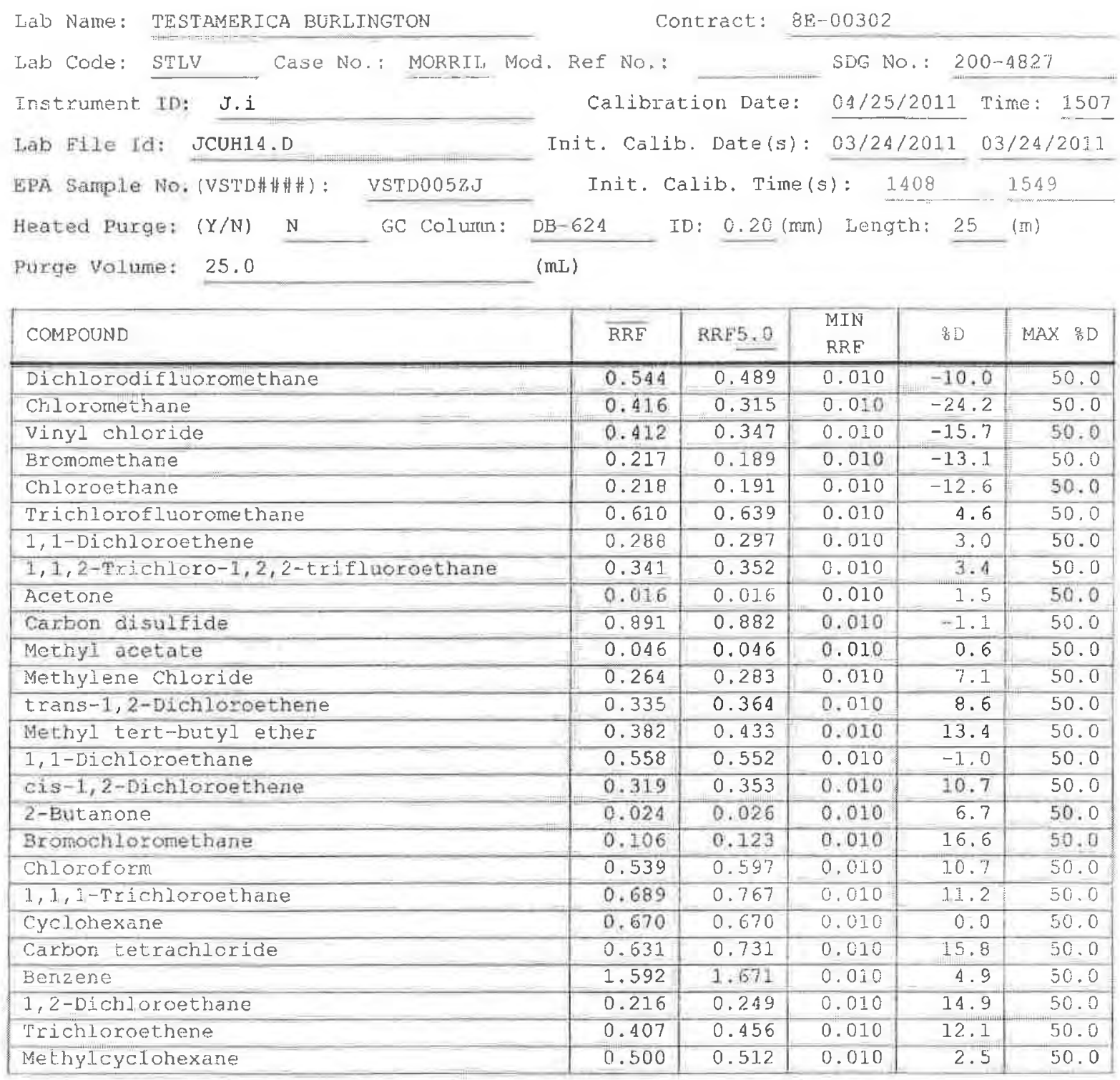

Report 1,4-Dioxane for Low/Medium VoA analysis onjy 
$7 B$ - FORM VII VOA-2

VOLATILE CONTTNUTHG CAITERAFION DATA

Lab Name: TESTAMERICA BURIINGTON

Contract: $\quad 8 \mathrm{E}-00302$

Lab Code: STLV Case No.: MORRIL Mod. Ref No.

SDG No.: $200-4827$

Instrument TD: J.i

Calibration Date: 04/25/2011 Time: 1507

Lab File Id: JCuH1A.D

EPA Saraple No. (VSTD\#\#\#\#): VSTD005\%J Init. Calib. Date(s): 03/24/2011 03/24/2011

Heated Purge: $(\mathrm{Y} / \mathrm{N}) \mathrm{N}$

GC Column: DB-624

Injt. Calib. Time (s): $1408 \quad 1549$

Purge volure: 25.0

(mis)

\begin{tabular}{|c|c|c|c|c|c|}
\hline COMPOUND & $\overline{\mathrm{RKF}}$ & RRF5.0 & $\begin{array}{l}\text { MIN } \\
\text { RRE }\end{array}$ & $\approx \mathrm{D}$ & $M A X \quad \& D$ \\
\hline 1,2-Dichloropropane & 0.311 & 0.309 & 0.010 & -0.9 & 50.0 \\
\hline Bromodichloromethane & 0.381 & 0.432 & 0.010 & 13.4 & 50.0 \\
\hline cis-1,3-Dichioropropene & 0.431 & 0.493 & 0.010 & 14.4 & 50.0 \\
\hline 4-Methyl-2-pentanone & 0.073 & 0.080 & 0.010 & 9.7 & 50.0 \\
\hline Toluene & 1.677 & 1.863 & 0.010 & 11.1 & 50.0 \\
\hline Erans-1,3-Dichloropropere & 0.306 & 0.371 & 0.010 & 21.2 & 50.0 \\
\hline $1,1,2$-Trichloroethane & 0.158 & 0.185 & 0.010 & 16.7 & 50.0 \\
\hline Tetrachloroethene & 0.357 & 0.404 & 0.010 & 13.1 & 50.0 \\
\hline 2-Hexancrie & 0.047 & 0.053 & 0.010 & 12.1 & 50.0 \\
\hline Dibromochloromethane & 0.216 & 0.273 & 0.010 & 26.5 & 50.0 \\
\hline 1,2-Dibromoethane & 0.140 & 0.167 & 0.010 & 19.7 & 50.0 \\
\hline Ch.1orobenzene & 1.008 & 1.139 & 0.010 & 13.0 & 50.0 \\
\hline Ethyloenzene & 1.856 & 2.074 & 0.010 & 11.8 & 50.0 \\
\hline o-xylene & 0.663 & 0.775 & 0.010 & 16.9 & 50.0 \\
\hline$m, p-x y l e n e$ & 0.713 & 0.830 & 0.010 & 16.4 & 50.0 \\
\hline Styrene & 0.969 & 1.187 & 0.010 & 22.5 & 50.0 \\
\hline Bromoform & 0.211 & 0.261 & 0.010 & 23.4 & 50.0 \\
\hline Isopropylbenzene & 1.828 & 2.117 & 0.010 & 15.8 & 50.0 \\
\hline $1,1,2,2-$ Tetrachlaroethane & $0 . \hat{1} 44$ & 0.160 & 0.010 & 11.8 & 50.0 \\
\hline 1,3-Dichlorobenzene & 1.528 & 1.755 & 0.010 & 14.9 & 50.0 \\
\hline I, a-Dich-orobenzene & 1.542 & 1.764 & 0.010 & 14.4 & 50.0 \\
\hline 1,2-Dichiorobenzene & 1.240 & 1.455 & 0.010 & 17.4 & 50.0 \\
\hline 1, 2-Dibromo-3-Chloropropare & 0.042 & 0.053 & 0.010 & 26.3 & 50.0 \\
\hline i,2,4-Trichlorobenzene & 0.712 & 0.869 & 0.010 & 22.0 & 50.0 \\
\hline $1,2,3$-Trichlorobenzene & 0.513 & 0.620 & 0.010 & 19.7 & 50.0 \\
\hline
\end{tabular}


$7 \mathrm{C}$ - FORM VII VOR-3

VOIATILE CONTINUING CALIBRATION DATA

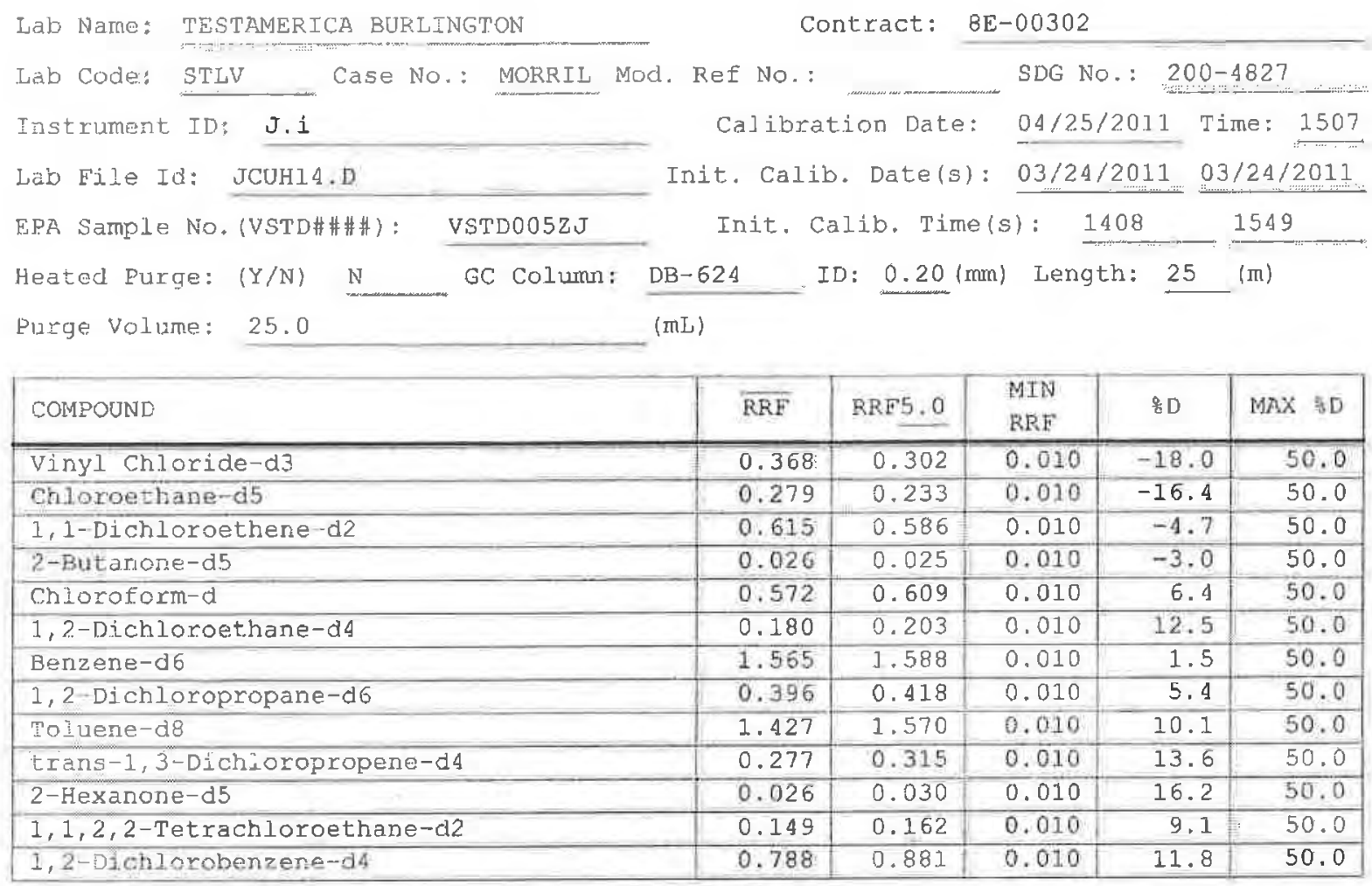

Report 1,4-Dioxane-d8 for Low/Medium VOA analysis only 
1A - FORM I VOA-1

EPA SAMPLE NO.

VOLATILE ORGANICS ANALYSIS DATA SHEET

VBLKJZ

Lab Name: TESTAMERICA BURLINGTON

Contract: 8E-00302

Eab Code: STLV Case No.: MORRIL Mod. Ref No.: SDG NO.: $200-4827$

Matrix: (SOIL/SED/WATER) Water

Sample wt/vol: $25.0 \quad(\mathrm{~g} / \mathrm{mL}) \mathrm{mL}$

Level: (TRACE/LOW/MED) TRACE

F Moisture: not dec.

GC Column: DB-624

IJ: $\quad 0.20$

(mm)

Soil Extract Volume:

(UI)

(mL)

Purge Volume: 25.0
Lab Sample ID: MB 200-16989/3

Lab File ID: ICUHO3.D

Date Received:

Date Analyzed: 04/25/2011

Dilution Factor: 1.0

Soil Aliquot Volume:

(uL)

\begin{tabular}{|c|c|c|c|}
\hline CAS NO. & COMPOUND & $\begin{array}{l}\text { CONCENTRATION UNITS: } \\
\text { (ug/L of } u g / \mathrm{kg}) \mathrm{ug} / \mathrm{L}\end{array}$ & $Q$ \\
\hline $75-7.1-8$ & Dichlorodifluoromethane & 0.50 & $\overline{\mathrm{U}}$ \\
\hline $74-87-3$ & Chloromethane & 0.047 & $\mathrm{~J}$ \\
\hline $75-01-4$ & Vinyl chloride & 0.50 & $\mathrm{U}$ \\
\hline $74-83-9$ & Bromornerhane & 0.50 & $\mathrm{U}$ \\
\hline $75-00-3$ & Chioroethane & 0.50 & 0 \\
\hline $75-69-4$ & "richlorofluoromethane & 0.50 & $\mathrm{U}$ \\
\hline $75-35-\varepsilon$ & 1,1-Dichloroethere & 0.50 & $\mathrm{u}$ \\
\hline $76-13-1$ & 1,1,2-Trichioro-1,2,2-trifluoroethane & 0.50 & प] \\
\hline $67-64-1$ & Acetone & 1.6 & $\bar{u}$ \\
\hline $75-15-0$ & Carbon distilfide & 0.20 & $J$ \\
\hline $79-20-9$ & Methyl acetate & 0.50 & 0 \\
\hline $75-09-2$ & Methylene Criloride & 0.047 & $\mathrm{~J}$ \\
\hline $156-60-5$ & trans-1,2-Dichl oroet hene & 0.50 & U \\
\hline $1634-04-4$ & Methyl tert-butyl ether & 0.50 & $\mathrm{U}$ \\
\hline $75-34-3$ & 1,1-Dichioroethane & 0.50 & $\bar{U}$ \\
\hline $156-59-2$ & cis-1,2-Dichloroethene & 0.50 & $\mathrm{U}$ \\
\hline $78-93-3$ & 2-Butanone & 5.0 & (1) \\
\hline $74-97-5$ & Bromochloromethane & 0.50 & $\mathrm{U}$ \\
\hline $67-66-3$ & ChIoroform & 0.50 & v \\
\hline $71-55-6$ & 1,1,1-Trichloroethane & 0.50 & 0 \\
\hline $110-82-7$ & Cyclohexane & 0.50 & D \\
\hline $56-23-5$ & Carbon tetrachloride & 0.50 & 0 \\
\hline $71-43-2$ & Benzene & 0.50 & $\mathrm{U}$ \\
\hline $107-106-2$ & $1,2-$ Dichloroethane & 0.50 & U \\
\hline
\end{tabular}

Report 1, A-Dioxane for Low-Medjum VoA analysis only 
EPA SAMPLE NO.

VOLATILE ORGANICS ANALYSIS DATA SHEET

VBLKJZ

Lab Name: TESTAMERICA BURLINGTON

Lab Code: STLV Case No.: MORRII Mod. Ref No.:

Matrix: (SOIL/SED/WATER)

Water

sample wt/vol: 25.0

$(\mathrm{g} / \mathrm{mL}) \quad \mathrm{mL}$

Leve1: (TRACE/LOW/MED) TRACE

Moisture: not dec.

GC Column: DB-624

ID: $0.20 \quad$ (mm)

Soil Extract Volume:

Purge Volume: 25.0

(uL) (mL)
Contract: $\quad 8 \mathrm{E}-00302$

SDG No.: 200-4827

\begin{tabular}{|c|c|c|c|}
\hline CAS NO. & COMPOUND & $\begin{array}{l}\text { CONCENTRATION UNITS: } \\
(\mathrm{ug} / \mathrm{L} \text { or } 11 \mathrm{~g} / \mathrm{kg}) \mathrm{ug} / \mathrm{L}\end{array}$ & $\mathrm{Q}$ \\
\hline $79-01-6$ & Trichloroethene & 0.049 & J \\
\hline $108-87-2$ & Methylcyclohexane & 0.50 & $\mathrm{U}$ \\
\hline $78-87-5$ & 1,2-Dichloropropane & 0.50 & U \\
\hline $75-27-4$ & Bromodichlorornethane & 0.50 & U \\
\hline $10061-01-5$ & cis-1,3-Dichloropropene & 0.50 & U \\
\hline $208-10-1$ & 4-Netbyl-2-pentarone & 5.0 & $\mathrm{U}$ \\
\hline $108-80-3$ & Toluene & 0.013 & $J$ \\
\hline $10061-02-6$ & trans-1,3-Dichloropropene & 0.50 & $\mathrm{U}$ \\
\hline $79-00-5$ & $1,1,2$-Trichloroetrane & 0.50 & $\mathrm{U}$ \\
\hline $127-18-4$ & Tetrachloroethene & 0.50 & U \\
\hline $591-78-6$ & 2-Hexanone & 5.0 & $\mathrm{U}$ \\
\hline $1,24-48-1$ & Dibromochloromethane & 0.50 & $\mathrm{D}$ \\
\hline $106-93-4$ & 1,2-Dibromoethane & 0.50 & $\mathrm{U}$ \\
\hline $108-90-7$ & Chlorobenzene & 0.50 & $\mathrm{U}$ \\
\hline $100-41-4$ & Ethylbenzene & 0.50 & D \\
\hline $95-47-6$ & o-xylene & 0.50 & is \\
\hline $179601-23-1$ & m, $\mathrm{p}-\mathrm{xyleme}$ & 0.50 & Q \\
\hline $100-42-5$ & Styrene & 0.50 & D] \\
\hline $75-25-2$ & Bromoform & 0.50 & U \\
\hline $98-82-8$ & Isopropy Ibenzene & 0.50 & $\mathrm{U}$ \\
\hline $79-34-5$ & $1,1,2,2$-Tetrachloroethane & 0.50 & u \\
\hline $541-73-\hat{i}$ & 1,3-Dichlorobenzene & 0.50 & 0 \\
\hline $106-46-7$ & 1,4-Djoblorobenzere & 0.50 & U \\
\hline $95-50-1$ & 1,2-Dichlorcionzene & 0.50 & $\mathrm{U}$ \\
\hline $96-12-8$ & 1,2-Dibromo-3-Chloropropane & 0.50 & U \\
\hline $120-82-1$ & $1,2,4$-Trichlorobenzene & $0.0 \overline{8}$ & $\mathrm{~J}$ \\
\hline $87-61-6$ & $1,2,3-$ Trichlorobenzene & 0.15 & $\mathrm{~J}$ \\
\hline
\end{tabular}

Lab Sample ID: MB 200-16989/3

Jab Eile ID: JCUH03.D

Date Received:

Date Analyzed: 04/25/2011

Dilution Factor: 1.0

Soil Aliquot Volume:

(uL) 
$1 \mathrm{~J}$ - FORM I VOA-TIC

VOLATILE ORGANICS ANALYSIS DATA SHEE'T TENTATIVELY IDENTIFIED COMPOUNDS
EPA SAMPLE NO.

VBLLKJZ

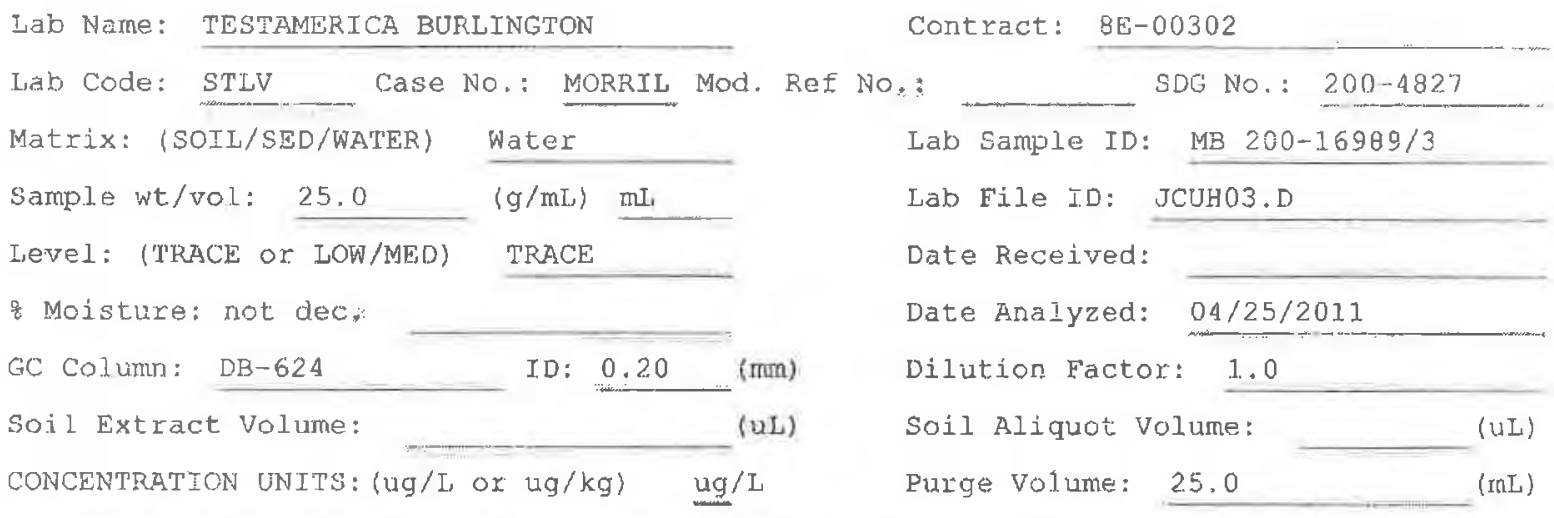

\begin{tabular}{|c|c|c|c|c|}
\hline CAS NUMBER & COMPOUND NAME & $\mathrm{RT}$ & EST. CONC: & Q \\
\hline & Dnknown & 4.13 & 2.8 & $\bar{J}$ \\
\hline & Unknown & 6.90 & 3.5 & $\mathrm{X} \mathrm{J}$ \\
\hline $541-05-9$ & Cyclotrisiloxane, hexamethyl- & 7.85 & 1.8 & $J N$ \\
\hline & Unknown siloxane derivative & 10.69 & 1.9 & $J$ \\
\hline & Unknown & 12.88 & 0.50 & ปี \\
\hline E966796? & Total Alkanes & $\tilde{N} / \bar{A}$ & & \\
\hline
\end{tabular}

1. EPA-designated Registry Number. 
EPA SAMPLE NO.

VHBLKOI

Läb Narre: TESTAMERICA BURLINGTON

Contract: $\quad 8 E-00302$

Lab Code: Smivy Case No.: MORRII Mod. Ref No.: SDG NO.: 200-4827

Matrix: (SOIL/SED/WATER) Water

Sample wt/vol: 25.0 (g/mL) $\mathrm{mL}$

Level: (TRACE/IOW/MED) TRACE

f Moisture: not dec.

GC Column: $\mathrm{DB}-624$ ID: 0.20 (mon)

Soil Extract Volume:

Purge Volume: 25.0 (mL)
Lab Sample ID: $200-4827-6$

Lab Eile ID: JCUH09.D

Date Received:

Date Analyzed: 04/25/2011

Dilution Factor: 1.0

Soil Aliguot Volume:

(uL)

\begin{tabular}{|c|c|c|c|}
\hline CAS NO. & COMPOUND & $\begin{array}{l}\text { CONCENTRATION UNITS: } \\
(\mathrm{ug} / \mathrm{L} \text { or } \mathrm{ug} / \mathrm{kg}) \mathrm{ug} / \mathrm{L}\end{array}$ & $Q$ \\
\hline $75-71-8$ & Diclulorodifluoromethane & 0.50 & $\bar{U}$ \\
\hline $74-87-3$ & Chloromethane & 0.50 & U \\
\hline $75-01-4$ & Vinyl chloride & 0.50 & U \\
\hline $74-83-9$ & Bromomethane & 0.50 & U \\
\hline $75-00-3$ & Chloroethane & 0.50 & $\mathrm{U}$ \\
\hline $75-69-4$ & Trichlorofluoromethane & 0.50 & $\mathrm{U}$ \\
\hline $75-35-4$ & 1,1-Dichloroethene & 0.50 & U \\
\hline $76-13-1$ & $1,1,2$-Trichloro-1,2,2-trifluoroethane & 0.50 & $\mathrm{U}$ \\
\hline $67-64-1$ & Acetone & 0.66 & J B \\
\hline $75-15-0$ & Carbon disulfide & 0.075 & $\mathrm{~J} \mathrm{~B}$ \\
\hline $79-20-9$ & Methyl acetate & 0.50 & $\mathrm{U}$ \\
\hline $75-09-2$ & Methylene Chloride & 0.50 & U1 \\
\hline $156-50-5$ & trans-1,2-Dichloroethene & 0.50 & $\mathrm{U}$ \\
\hline $1634-04-4$ & Methyl tert-butyl ether & 0.50 & $\mathrm{U}$ \\
\hline $75-34-3$ & 1,1-Dichloroethane & 0.50 & U \\
\hline $156-59-2$ & cis-1,2-Dichloroethene & 0.50 & E \\
\hline $78-93-3$ & 2-Butanone & 5.0 & Y! \\
\hline $74-97-5$ & Bromochloromethare & 0.50 & ए1 \\
\hline $67-66-3$ & Chloroform & 0.50 & U \\
\hline $71 \cdot-55-6$ & $1,1,1-$ Trichloroethane & 0.50 & UI \\
\hline $110-82-7$ & Cyclonexane & 0.50 & $\mathrm{U}$ \\
\hline $56-2.3-5$ & Carbon tetrachloride & 0.50 & $\mathrm{U}$ \\
\hline $71-43-2$ & Benzene & 0.50 & U \\
\hline $107-06-2$ & 1,2-Dichlorocthane & 0.50 & $\mathrm{U}$ \\
\hline
\end{tabular}

Report 1, 4-Dioxane for Low-Medium voA analysis only 
1B - FORM I VOA-2

VOLATILF ORGANTCS ANALYSIS DATA SHEET
EPA SAMPLE NO.

VHBLKO1
Lab Name: TESTAMERICA BURLINGTON

Lab Code: STLV

Matrix: (SOIL/SED/WATER)

Sample wt/vol: 25.0

Level: (TRACT/LOW/MED)

Case No.: MORRIJ Mod. Ref No.:

Contract: $\quad 8 E-00302$

o Molsture: not dec.

GC CoIumn: DB-624

Soil Extract Volume: Water

$(g / \mathrm{mL})$ mI TRACE

Purge Volume: 25.0

\begin{tabular}{|c|c|c|c|}
\hline CAS NO. & COMPOUND & $\begin{array}{l}\text { CONCENTRATION UNITS: } \\
(u g / L \text { or } u g / \mathrm{kg}) \mathrm{ug} / \mathrm{L}\end{array}$ & $Q$ \\
\hline $79-01-6$ & Trichloroethene & 0.50 & $\overline{0}$ \\
\hline $108-87-2$ & Methylcyclohexane & 0.50 & $\mathrm{u}$ \\
\hline $78-87-5$ & 1,2-Dichloropropane & 0.50 & $\mathrm{U}$ \\
\hline $75-27-4$ & Bromodichloromethane & 0.50 & $\mathrm{U}$ \\
\hline $10061-01-5$ & cis-1,3-Dichloropropene & 0.50 & $\mathrm{u}$ \\
\hline $108-10-1$ & 4-Methyl-2-pentanone & 5.0 & 0 \\
\hline $108-88-3$ & Toluene & 0.50 & y \\
\hline $10061-02-6$ & trans $-1,3-$ Dichloropropene & 0.50 & $\mathrm{U}$ \\
\hline $79-00-5$ & 1,1,2-Trichloroethane & 0.50 & U \\
\hline $127-18-4$ & Tetrachloroethene & 0.50 & $\mathrm{U}$ \\
\hline $591-78-6$ & 2-Hexanone & 5.0 & 0 \\
\hline $124-48-1$ & Dibromochloromethane & 0.50 & U \\
\hline $106-93-4$ & 1,2-Dibromoethane & 0.50 & $\mathrm{U}$ \\
\hline $108-90-7$ & Chlorobenzene & 0.50 & $\mathrm{U}$ \\
\hline $100-41-4$ & Ethylberizene & 0.50 & U \\
\hline $95-47-6$ & o-xyIene & 0.50 & $\mathrm{U}$ \\
\hline $179601-23-1$ & m,p-Xylene & 0.50 & U \\
\hline $100-42-5$ & Styrene & 0.50 & U \\
\hline $75-25-2$ & Bromoform & 0.50 & $\overline{1}$ \\
\hline $98-82-8$ & Isopropylbenzene & 0.50 & $\mathrm{O}$ \\
\hline $79-34-5$ & 1,1,2,2-Tetrachloroethane & 0.50 & $\mathrm{U}$ \\
\hline $541-73-1$ & 1, 3-Dichlorobenzene & 0.50 & $\mathrm{U}$ \\
\hline $106-96-7$ & 1.4-Dichlorobenzene & 0.50 & 0 \\
\hline $95-50-1$ & 1,2-Dichlorobenzene & 0.50 & $\mathrm{U}$ \\
\hline $96-12-8$ & 1,2-Díbromo-3-Chloropropane & 0.50 & $\mathrm{U}$ \\
\hline $120-82-1$ & 1,2,4-Trichlorobenzene & 0.50 & 0 \\
\hline $87-61-6$ & $1, \overline{2}, 3$-Trichlorobenzene & 0.50 & U \\
\hline
\end{tabular}

Lab File ID: JCUHO9.D

Date Received:

Date Analyzed: $04 / 25 / 2011$

Dilution Factor: 1.0

Soli. Alliquot Volume: (uL)
Lab Sample ID: 200-4827-6 (aL)

(mL) SDG No.: $200-4827$ 
$1 \mathrm{~J}$ - FORM I VOA-TIC

VOLATILE ORGANICS ANALYSIS DATA SHEET

TENTATIVELY IDENTIFIED COMPOUNDS
EPA SAMPLE NO. VHBLKO1

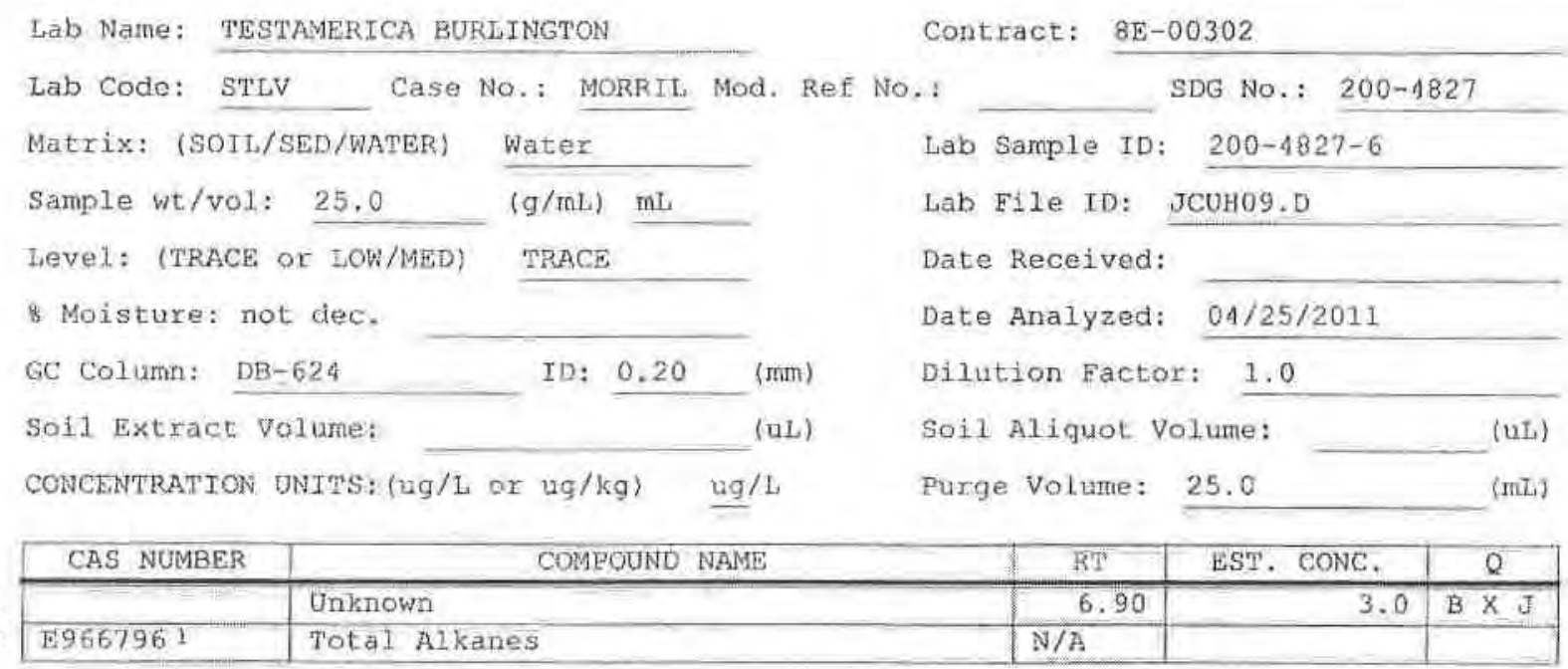

1. EPA-designated Registry Number; 


\section{TestAmerica}

THE LEADER IN ENYIRDNMENTAL TESTING

\section{ANALYTICAL REPORT}

Job Number: 200-7357-1

SDG Number: 200-7357

Job Description: Morrill (200-7357)

Contract Number: 1E-30401

For:

Argonne National Laboratory 9700 South Cass Avenue

Building 203

Office B-149

Argonne, IL 60439

Attention: Mr. Clyde Dennis

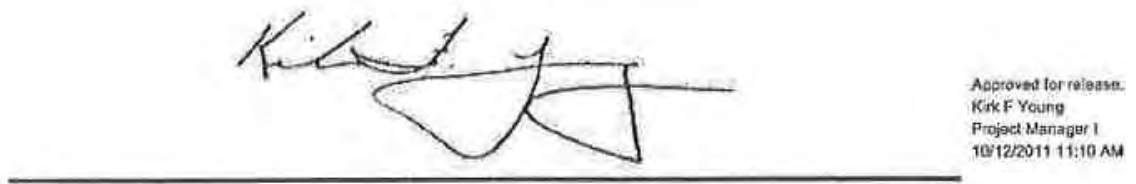

Kirk F Young

Project Manager I

kirk.young@testamericainc.com

$10 / 12 / 2011$

The test results in this report relate only to sample(s) as received by the laboratory. These test results were derived under a quality system that adheres to the requirements of NELAC. Pursuant to NELAC, this report may not be produced in full without written approval from the laboratory 


\section{Table of Contents}

Cover Title Page ......................... 1

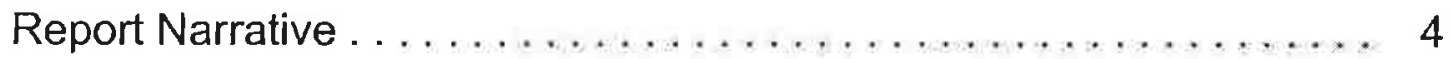

Case Narrative $\ldots \ldots \ldots \ldots \ldots \ldots \ldots \ldots \ldots \ldots \ldots \ldots \ldots \ldots, 4$

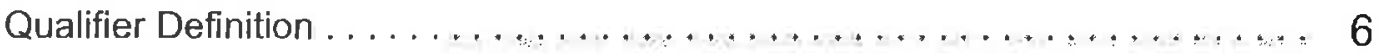

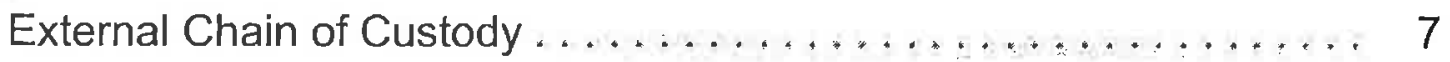

Internal Chain of Custody $\ldots \ldots \ldots \ldots \ldots, \ldots \ldots \ldots \ldots \ldots, 8$

Shipping Documentation . . . . . . . . . . . . . . . . . . . . 10

Airbills (if Applicable) . ............................ 11

Sample Receipt and Log In Check List . . ., .................... 12

Methodology Review . . . . . . . . . .................... 14

QC Summary - SOM01.2 Volatiles-Trace . . . . . . . . . . . . . . . . . 15

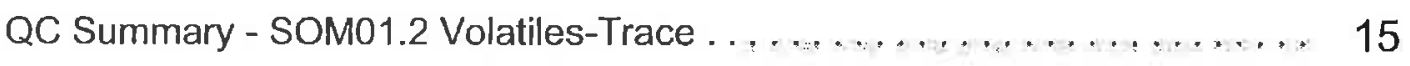

Deuterated Monitoring Compound Summary . . ..................... 15

Method Blank ......................................, 17

GC/MS Instrument Performance Check ......................... 18

Internal Standard Area and RT Summary ........................ 20

Sample Data - SOM01.2 Volatiles-Trace . . . . . . . . . . . . . . . 21

Sample Data - SOM01.2 Volatiles-Trace . . .................. 21

MRMW11S-W-30078................................ 21

MRMW2S-W-30069..................................... 27

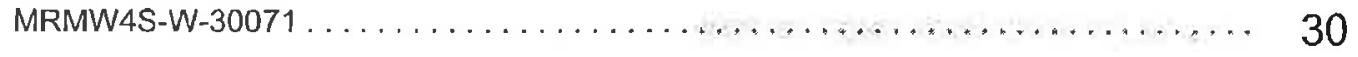

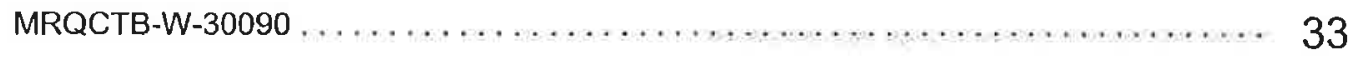

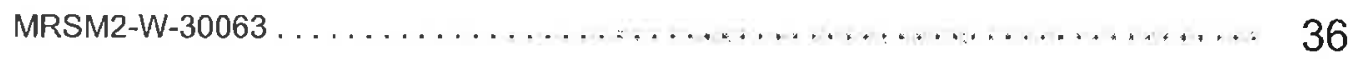

Standards - SOM01.2 Volatiles-Trace . . . . . . . . . . . . . . . 39

Standards - SOM01.2 Volatiles-Trace . . . . . . . . . . . . . . . . . 39

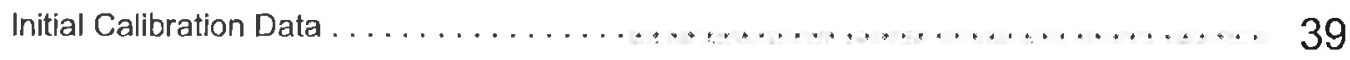




\section{Table of Contents}

CCV Data, including closing CCV ..........

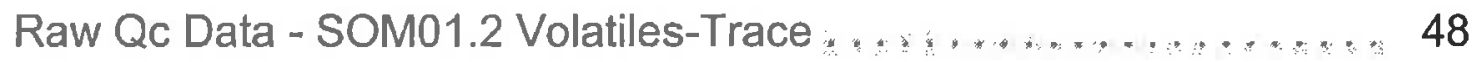

Raw Qc Data - SOM01.2 Volatiles-Trace ..... 48

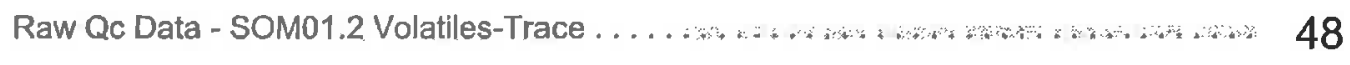

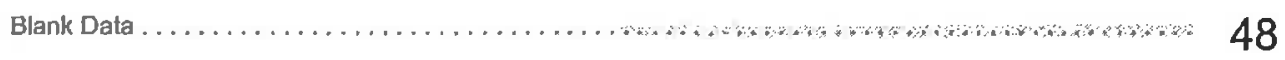




\title{
CASE NARRATIVE
}

\author{
Client: Argonne National Laboratory \\ Project: Morrill (200-7357)
}

Report Number: 200-7357-1

\begin{abstract}
Enclosed is the data set for the referenced project work. With the exceptions noted as flags or footnotes, standard analytical protocols were followed in performing the analytical work and the applied control limits were met.
\end{abstract}

Calculations were performed before rounding to avoid round-off errors in calculated results.

All holding times were met and proper preservation noted for the methods performed on these samples, unless otherwise detailed in the individual sections below.

\section{Receipt}

The samples were received on 10/05/2011. Documentation of the condition of the samples at the time of their receipt and any exception to the laboratory's Sample Acceptance Policy is documented in the Shipping and Receiving section of this submittal. The samples, as received, were not acid preserved. On that basis, the laboratory did provide for the analytical work to be performed within seven days of sample collection.

\section{SOM01.2 Volatile Organics (Trace Level Water)}

A storage blank was prepared for volatile organics analysis, and stored in association with the storage of the samples. That storage blank, identified as VHBLK01, was carried through the holding period with the samples, and analyzed.

Each sample in the sample set was analyzed without a dilution. An additional, dilution analysis was performed on sample MRMW11S-W-3007 in order to provide quantification within the range of calibrated instrument response. Both sets of results for the analysis of sample MRMW11S-W-3007 are included in this submittal:

Each of the analyses associated with the sample set exhibited an acceptable internal standard performance. There was an acceptable recovery of each deuterated monitoring compound (DMC) in the analysis of the method blank associated with the analytical work, and in the analysis of the storage blank associated with the sample set. The analysis of the samples in this sample set did meet the technical acceptance criteria specific to DMC recoveries, although not all DMC recoveries were within the control range in each analysis. The technical acceptance criteria does provide for the recovery of up to three DMCs to fall outside of the control range in the analysis of field samples. Matrix spike and matrix spike duplicate analyses were not performed on samples in this sample set. Trace concentrations of bromomethane, acetone, carbon disulfide, methylene chloride, carbon tetrachloride, toluene, $m, p$-xylene, 1,3-dichlorobenzene, 1,2,4-trichlorobenzene, and 1,2,3-trichlorobenzene were identified in the analysis of the method blank associated with the analytical work. The concentration of each analyte in that analysis was below the established reporting limit, and the analysis did meet the technical acceptance criteria for a compliant method blank analysis. Trace concentrations of carbon disulfide and carbon tetrachloride were identified in the analysis of the storage blank associated with the sample set. The concentration of each analyte in that analysis was below the established reporting limit, and the analysis did meet the 
technical acceptance criteria for a compliant storage blank analysis. Trace concentrations of carbon disulfide, carbon tetrachloride, and toluene were identified in the analysis of the instrument blank associated with the analytical work. The concentration of each analyte in that analysis was below the established reporting limit, and the analysis did meet the technical acceptance criteria for a compliant instrument blank analysis. Present in the method blank, instrument blank, and storage blank analyses was a non-target constituent that represents a compound that is related to the DMC formulation. The fact that the presence of this compound is not within the laboratory's control is at issue. The derived results for that compound have been qualified with an "X" qualifier to reflect the source of the contamination.

The responses for each of the target analytes met the relative standard deviation criterion in the initial calibration. The response for each target analyte met the percent difference criterion in the opening/continuing calibration check acquisition. The response for each target analyte met the 50.0 percent difference criterion in the closing calibration check acquisition.

The primary quantitation mass for methylcyclohexane that is specified in the Statement of Work is mass 83 . The laboratory did identify a contribution to mass 83 from 1,2-dichloropropane-d $d_{6}$, one of the deuterated monitoring compounds (DMCs). The laboratory did change the primary quantitation mass assignment to mass 55 for the quantification of methylcyclohexane.

Manual integration was employed in deriving certain of the analytical results. The values that have been derived from manual integration are qualified on the quantitation reports. Extracted ion current profiles for each manual integration are included in the data package, and further documented at the end of this submittal. 


\section{DATA REPORTING QUALIFIERS}

Client: Argonne National Laboratory

Job Number: $200-7357-1$

Sdg Nümber: $200-7357$

GC/MS VOA

U

E

$J$

$J$

D

$x$

B
Analyzed for but not detected.

Compound concentration exceeds the upper level of the calibration range of the instrument for that specific analysis.

Indicates an Estimated Value for TICs

Indicates an estimated value.

Sample was analyzed at a higher dilution factor.

See case narrative notes for explanation of the ' $X$ ' flag

Surrogate exceeds the control limit

The analyte was found in an associated blank, as well as in the sample. 


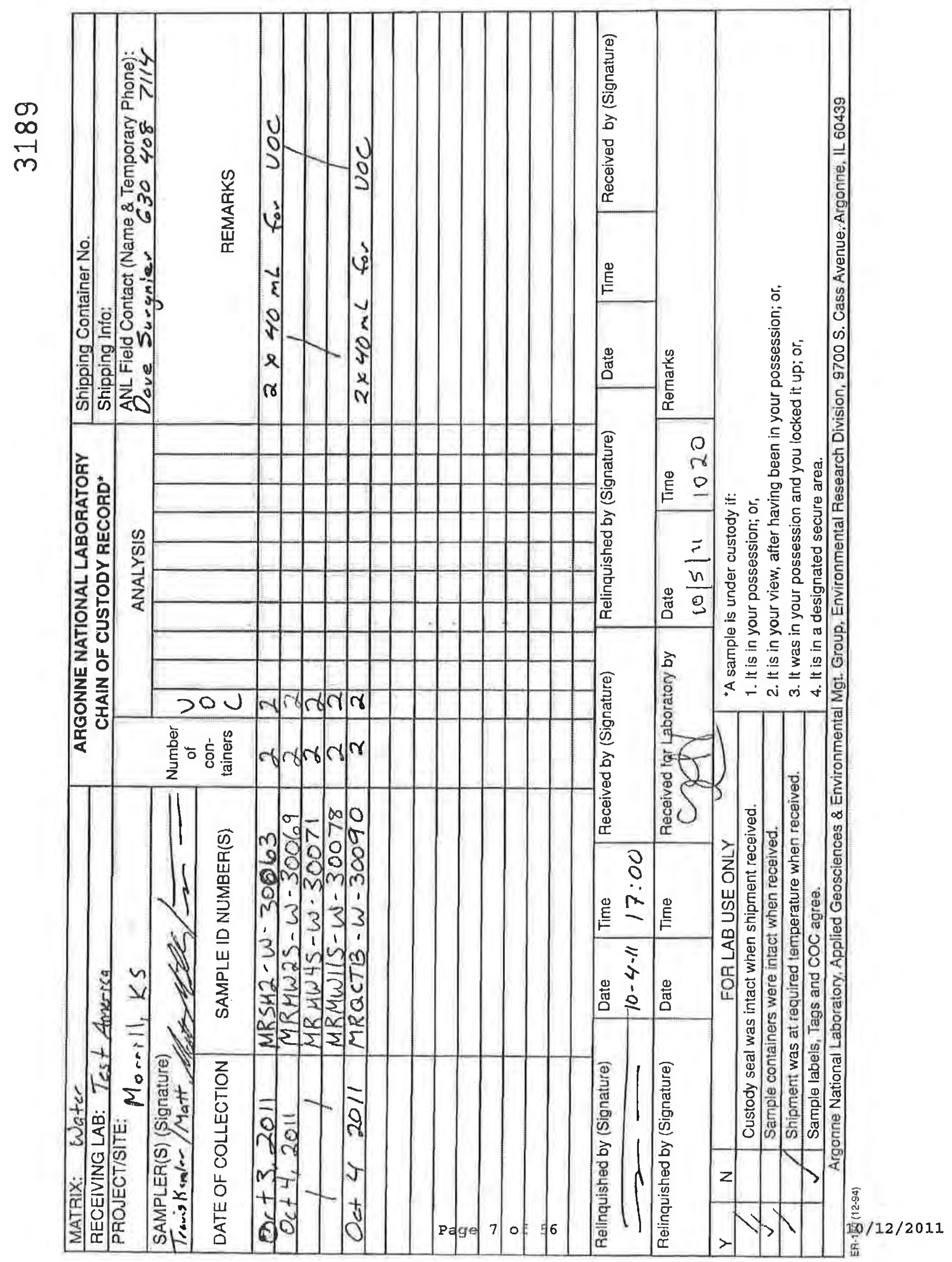




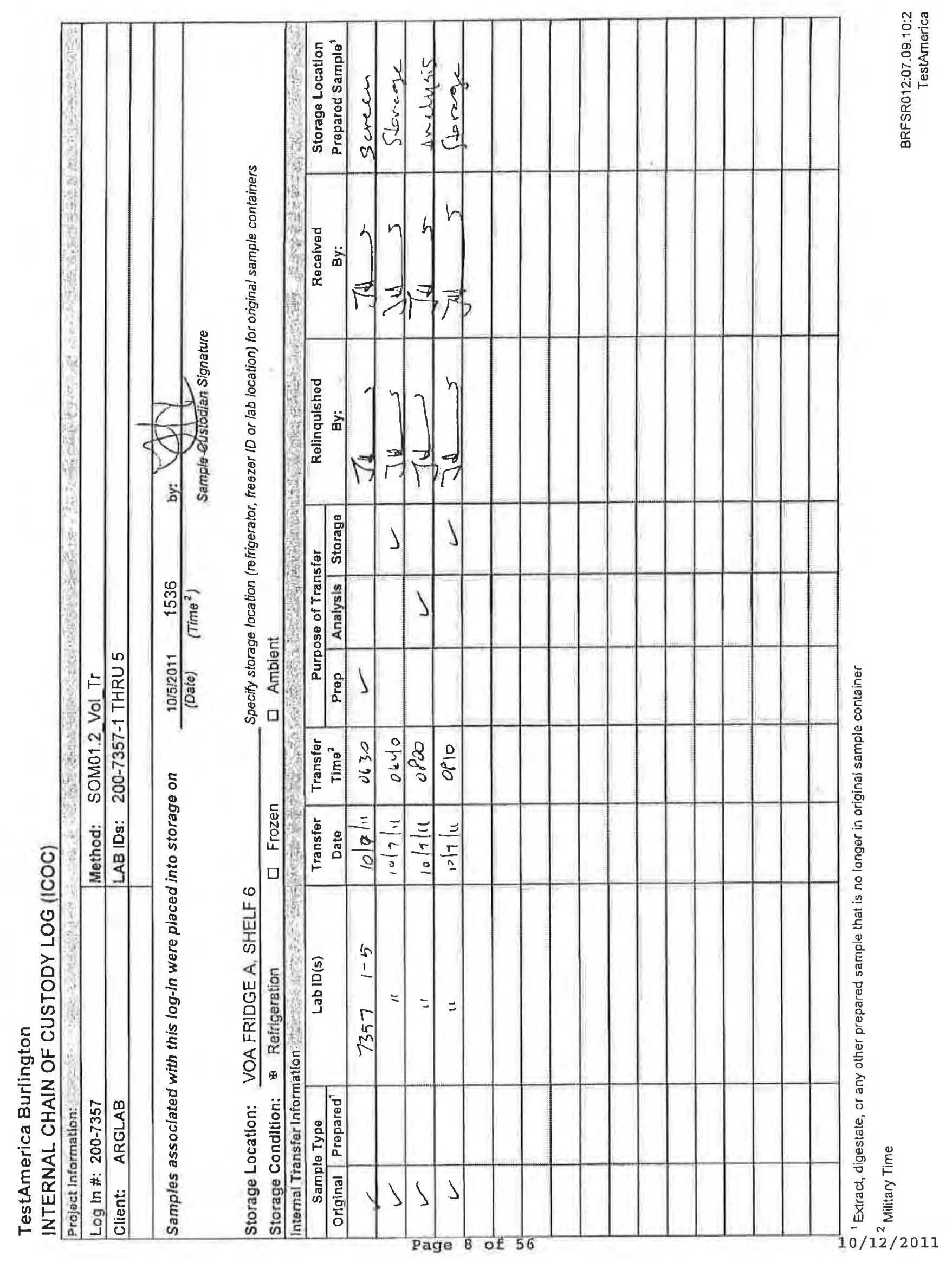




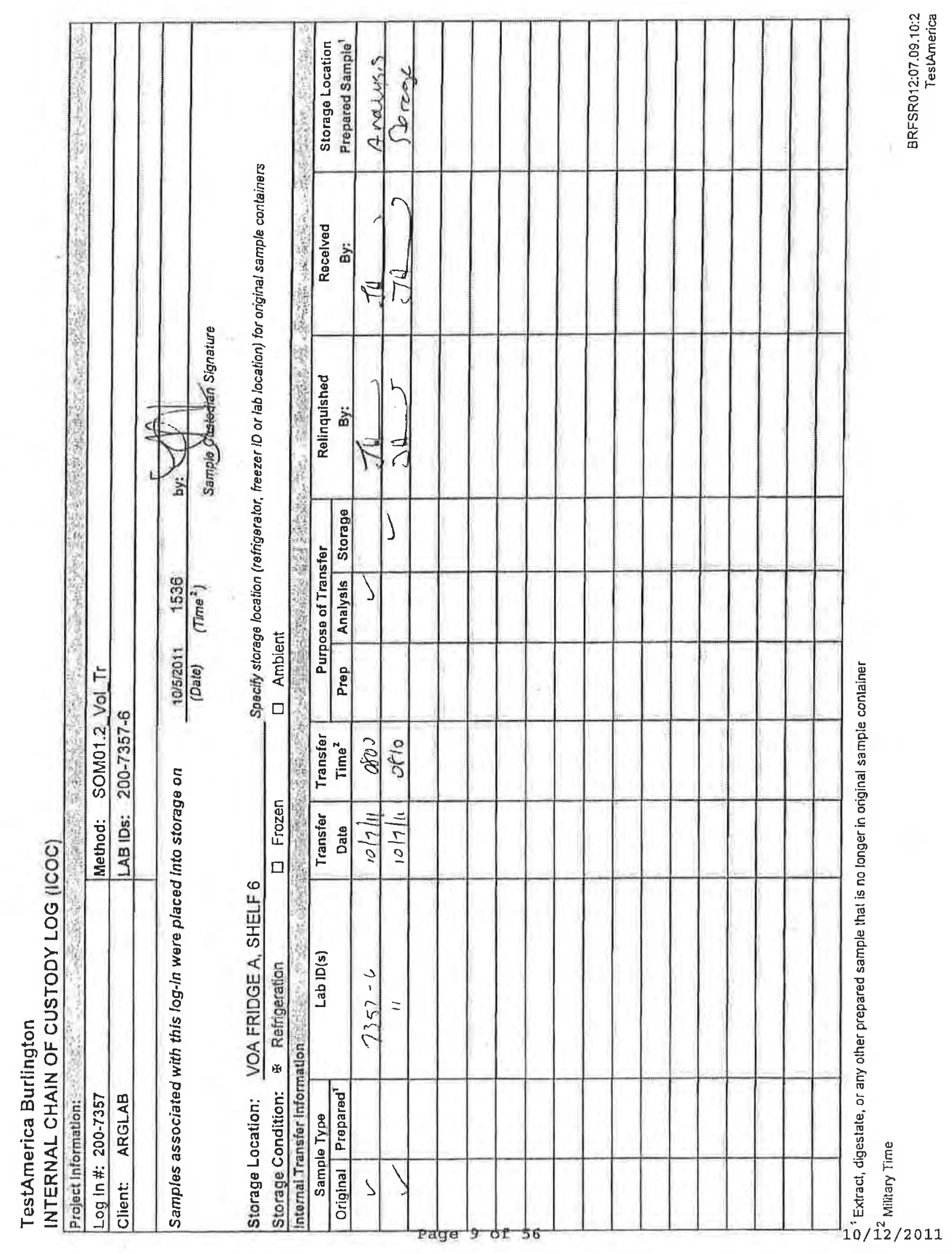




\section{Shipping and Receiving Documents}




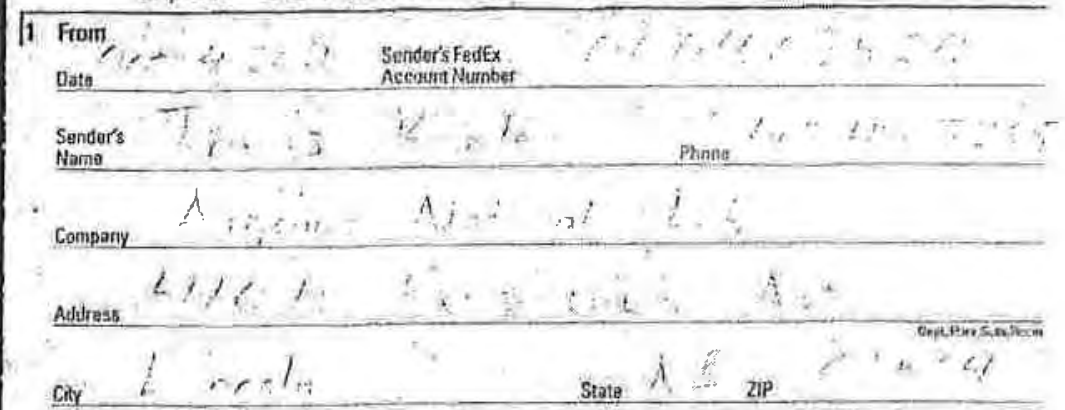

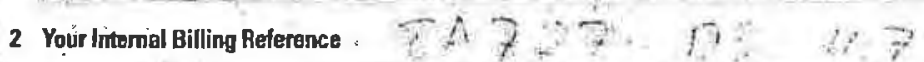

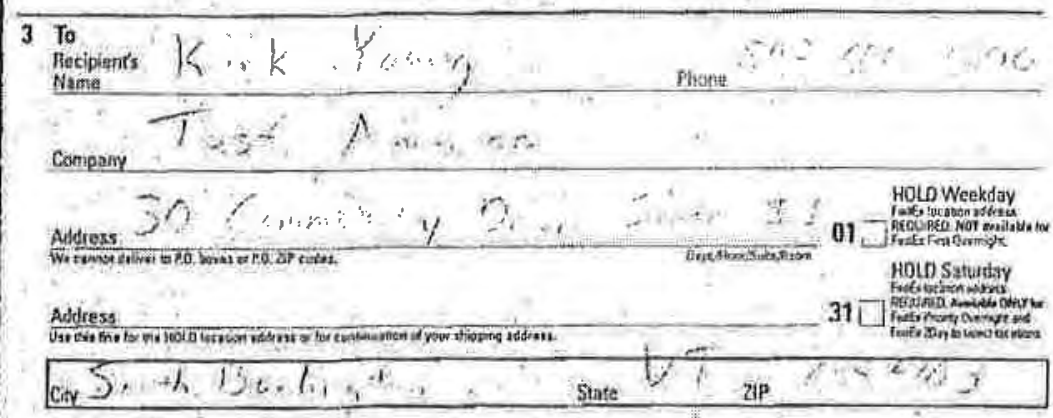

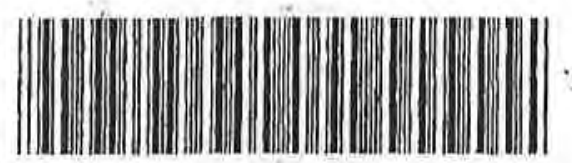

87592181280

\begin{tabular}{|c|c|c|}
\hline 4 & 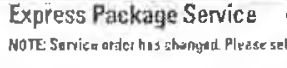 & 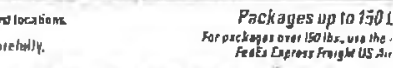 \\
\hline & 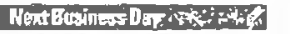 & 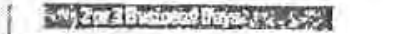 \\
\hline 06 & 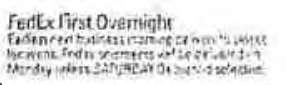 & 49 NEWFedex 20ar $1: 4$ \\
\hline 01 t) & 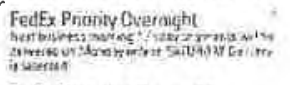 & 03 - Foder 20dy \\
\hline $055^{-}$ & 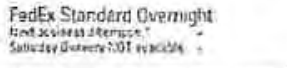 & 20 - FodEx Express Saver \\
\hline
\end{tabular}

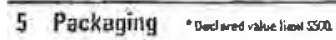

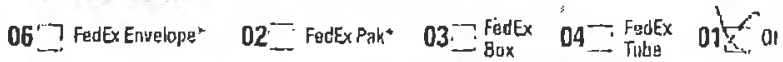

6 Special Handling and Delivery Signature Opțions 03:- SATURDAY DELUVERY

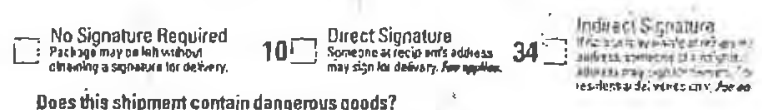

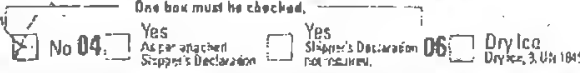

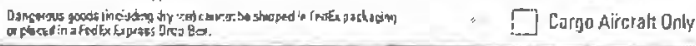
7 Payment Bilic:

Celain recip.
ñer. No.

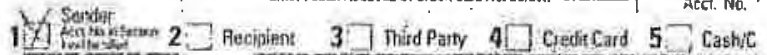

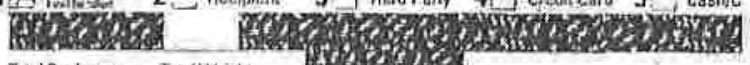

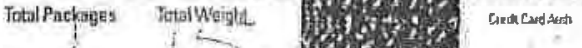

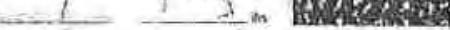




\section{Login Sample Receipt Checklist}

Creator: Holt, Jamie

\begin{tabular}{|c|c|c|}
\hline Question & Answer & Comment \\
\hline $\begin{array}{l}\text { Radioaclivity either was not measured or, if measured, is at or below } \\
\text { background }\end{array}$ & N/A & Lab does not accept radioaclive samples. \\
\hline The cooler's custody seal, if present, is intact. & True & NO CUSTODY SEAL NUMBERS \\
\hline $\begin{array}{l}\text { The cooler or samples do not appear to have been compromised or } \\
\text { tampered with. }\end{array}$ & True & \\
\hline Samples were received on ice. & True & \\
\hline Cooler Temperature is acceptable. & True & \\
\hline Cooler Temperature is recorded. & True & $2.6^{\circ} \mathrm{C}$, IR GUN ID 96, CF 0 \\
\hline $\mathrm{COC}$ is present. & True & \\
\hline COC is filled out in ink and legible. & True & \\
\hline COC is filled out with all pertinent infornation. & True & \\
\hline Is the Field Sampler's name present on COC? & True & \\
\hline $\begin{array}{l}\text { There are no discrepancies between the sample IDs on the containers and } \\
\text { the COC. }\end{array}$ & True & \\
\hline Samples are received within Holding Time. & True & \\
\hline Sample containers have legible labels. & False & $\begin{array}{l}\text { Both vials for one sample not labled. Sample ID } \\
\text { assigned by elimination process. }\end{array}$ \\
\hline Containers are not broken or leaking. & True & \\
\hline Sample colleclion date/times are provided. & True & \\
\hline Appropriate sample containers are used. & True & \\
\hline Sarnple bottles are completely filled. & True & \\
\hline Sample Preservation Verified. & N/A & Sample volumes were received unpreserved. \\
\hline $\begin{array}{l}\text { There is sufficient vol. for all requested analyses, incl. any requested } \\
\text { MS/MSDs }\end{array}$ & True & \\
\hline $\begin{array}{l}\text { VOA sample vlals do nol have headspace or bubble is }<6 \mathrm{~mm}\left(1 / 4^{\prime \prime}\right) \text { in } \\
\text { diameter. }\end{array}$ & True & \\
\hline Multiphasic samples are not present. & True & \\
\hline Samples do not require splitting or compositing. & True & \\
\hline Residual Chlorine Checked. & N/A & \\
\hline
\end{tabular}




\section{Sample Login Acknowledgement}

Job 200-7357-1

$\begin{array}{ll}\text { Client Job Description: } & \text { Morrill (200-7357) } \\ \text { Purchase Order \#: } & 1 \mathrm{E}-30401 \\ \text { Work Order \#: } & 1 \mathrm{E}-30401 \\ \text { Project Manager: } & \text { Kirk F Young } \\ \text { Job Due Date: } & \text { 10/19/2011 } \\ \text { Job TAT: } & 14 \text { Days } \\ \text { Max Deliverable Level: } & \text { IV } \\ & \\ \text { Earliest Deliverable Due: } & 10 / 19 / 2011\end{array}$

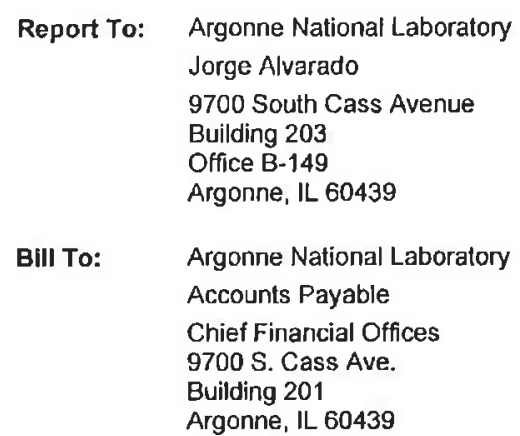

Login 200-7357

\section{Sample Recelpt:}

Method of Delivery:

\begin{tabular}{|c|c|}
\hline $\begin{array}{l}\text { Lab Sample \# } \\
\text { Method }\end{array}$ & $\begin{array}{l}\text { Client Sample ID } \\
\text { Method Description / Work Location }\end{array}$ \\
\hline $200-7357-1$ & MRSM2-W-30063 \\
\hline SOM01.2_Vol_Tr & SOM01.2 Trace Volatile Organics / In-Lab \\
\hline 200-7357-2 & MRMW2S-W-30069 \\
\hline SOM01.2_Vol_Tr & SOM01.2 Trace Volatile Organics / In-Lab \\
\hline $200-7357-3$ & MRMW4S-W-30071 \\
\hline SOM01.2_Vol_Tr & SOM01.2 Trace Volatile Organics / In-Lab \\
\hline $0-7357-4$ & MRMW11S-W-30078 \\
\hline SOM01.2_Vol_Tr & SOM01.2 Trace Volatlle Organics / In-Lab \\
\hline 200-7357-5 & MRQCTB-W-30090 \\
\hline SOM01.2_Vol_Tr & SOM01.2 Trace Volallie Organics / In-Lab \\
\hline $00-7357-6$ & VHBLKO1 \\
\hline SOM01.2_Vol_Tr & SOM01.2 Trace V \\
\hline
\end{tabular}

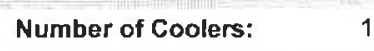




\section{METHODOLOGY SUMMARY}

\begin{tabular}{llll} 
Laboratory: & TestAmerica Laboratories & Project No: & \\
Location: & South Burlington, Vermont & SDG No: & $200-7357$ \\
\hline VOA & & \\
Volatile Organics Trace - USEPA CLP SOM01.2 & \\
\hline
\end{tabular}


$2 A$ - EORM II VOA-1

WATER VOLATILE DEUTERATED MONITORING COMPOUND RECOVERY

Lab Name: TESTAMERICA BURLINGTON

Contract: $\quad 8 \mathrm{E}-00302$

Lab Code: STLV Case No.: MORRIL Mod. Ref No:

SDG NNo.: 200-7357

Level: (TRACE or LOW) TRACE

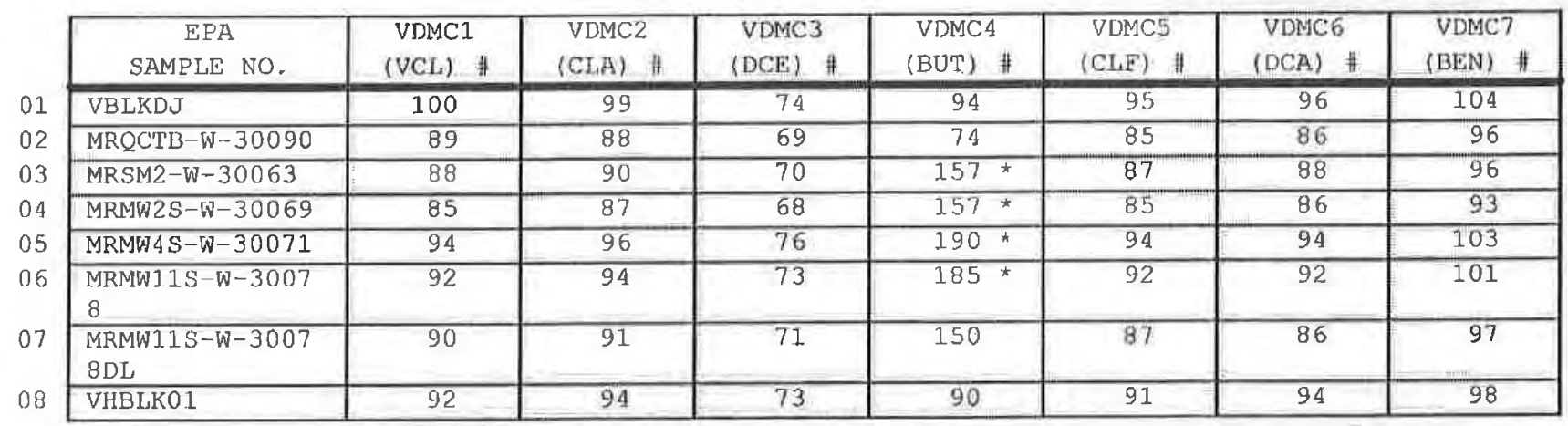

\footnotetext{
VDMC1 (VCL) = Vinyl Chloride-d3

VDMC2 $(C D A)=$ Chloroethane-d5

VDMC3 $(D C E)=1,1-D i c h l o r o e t h e n e-d 2$

VDMCA (BUT) = 2-Butanone-d5

VDMC5 $(\mathrm{CIE})=$ Chloroform $-\mathrm{d}$

VDMC6 $($ DCA $)=1,2-$ Dichloroethane-d 4

VDMC7 (BEN) = Benzene-d 6
}

$$
\begin{gathered}
\text { QC LIMITS } \\
\hline(65-131) \\
(71-131) \\
(55-104) \\
(49-155) \\
(78-121) \\
(78-129) \\
(77-124)
\end{gathered}
$$

\# Colum to be used to Elag recovery values

* Values outside of contract required QC limits 
$2 B$ - FORM II VOA-2

WATER VOLATILE DEUTERATED MONITORTNG COMPOUND RECOVERY

IJab Name: TESTAMERICA BURLINGTON

Contract: $8 \mathrm{E}-00302$

Lab Code: STLV Case No, : MORRIL Mod. ReE No.:

SDG No.: $200-7357$

Level: (TRACE Or LOW) TRACE

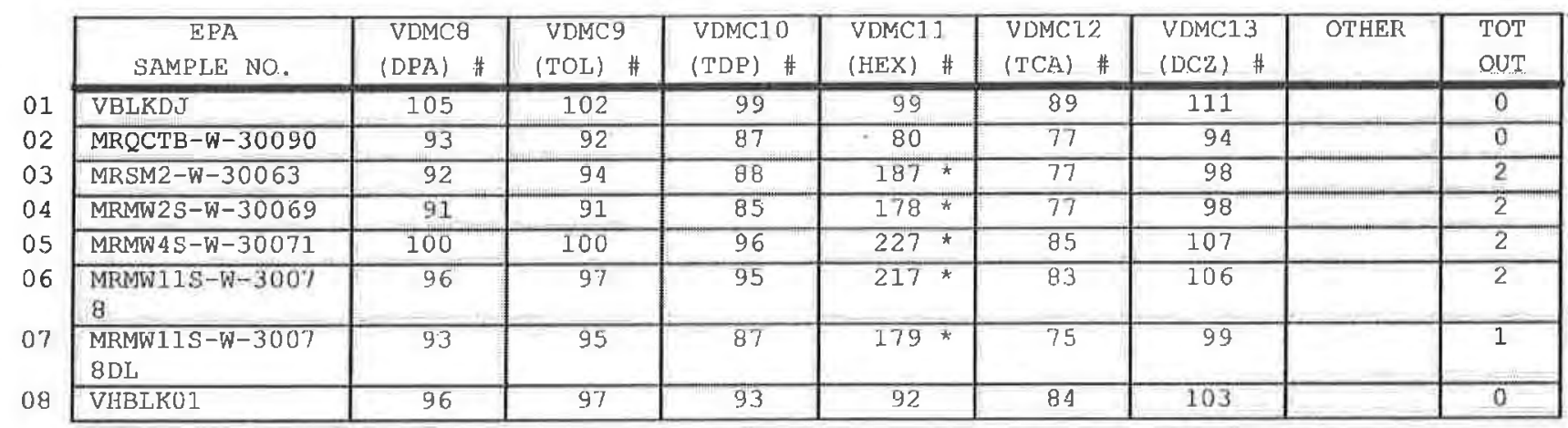

\footnotetext{
VDMC8 (DPA) 1,2-Dichloropropane-d6

VDMC9 (TOL) $\rightleftharpoons$ Toluene $-d 8$

VDMC10 (TDP) $\Rightarrow$ trans-1,3-Dichloroptopene-d4

VDMC11 $($ HEX $)=2$-Hexanone-d5

VDMC12 $($ TCA $)=1,1,2,2$-Tetrachloroethane-d2

VDMC13 $(\mathrm{DCZ})=1,2-$ Dichlorobenzene-dA
}

$$
\begin{gathered}
\text { QC LIMITS } \\
\hline(79-124) \\
(77-121) \\
(73-121) \\
(28-135) \\
(73-125) \\
(80-131)
\end{gathered}
$$

\# Columin to be used to flag recovery values

* Values outside of contract required oC Iimits

Report 1,1-Dioxane-d8 for Low-Medium VOA analysis only

Page 1 of 1 
$4 A-$ FORM IV VOA

VOLATILE METHOD BIAANK SUMMARY
EPA SAMPLE NO.

VBLKDJ
Lab Name: 'IESTAMERICA BURLINGTON

Lab Code: STLV........ Case No.: MORRIL Mod. Ref No.:

Contract: $8 \mathrm{E}-00302$

SDG No.: 200-7357

Lab rile ID: DHTA03.D

Instrument ID: D. i.

Matrix: (SOIL/SED/WATER)

Level: (TRACE or LOW/MED)

GC Column: DB-624

\section{Water}

TRACE

ID: 0.20
Lab Sample ID: MB 200-26380/3

Date Analyzed: 10/07/2011

Time Analyzed: 0754

Heated Purge: (Y/N) N

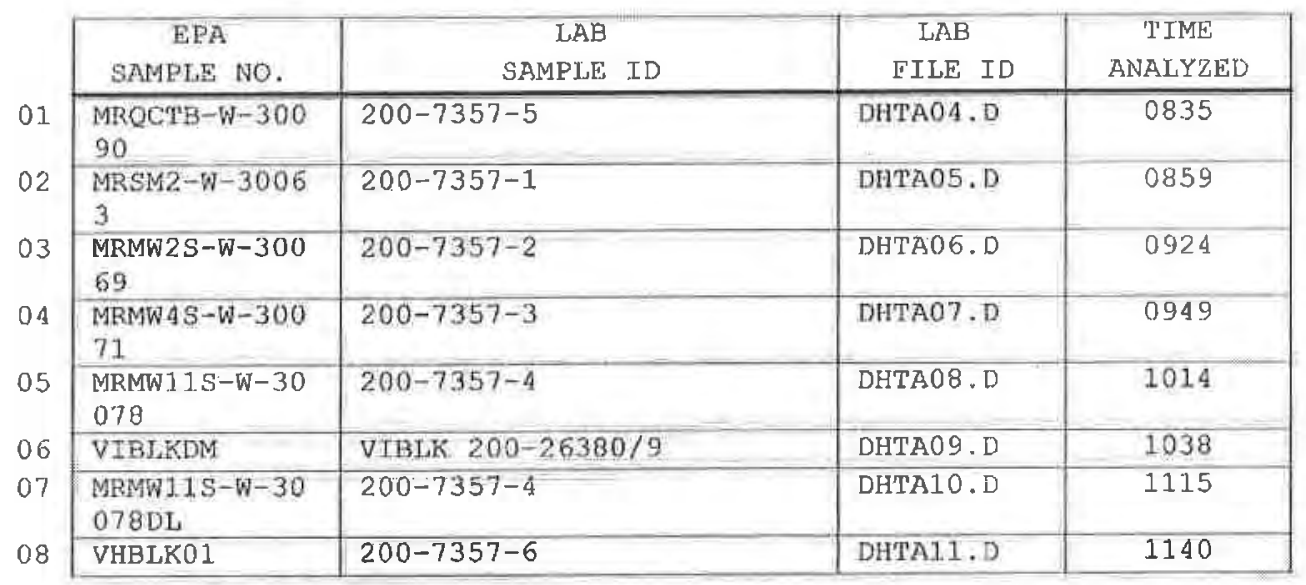

COMMENTS : 
$5 A$ - FORM V VOA

VOI,ATILE ORGANICS INSTRUMENT

PEREORMANCE CHECK

BROMOFLUOROBENZENE (BTB)
EPA SAMPLE NO.

BFBDI
Lab Name: TESTAMERICR BURLINGTON

Lab code: STLV Case No,: MORRIL Mod. Ref No.:
Contract: $\quad 8 \mathrm{~B}-00302$

BFB Injection Date: 10/06/2011

BFB Injection Time: 1158

SDG NO.: 200-7357
Instrument Id: D.i

(min)

Lab File Id: DHT01.D

GC Column: $\mathrm{DB}-624$

\begin{tabular}{|c|c|c|}
\hline $\mathrm{m} / \mathrm{e}$ & ION ABUNDANCE CRITERIA & $\begin{array}{l}\text { RESLATIVE } \\
\text { ABUNDANCE }\end{array}$ \\
\hline 50 & $15.0-40.0 \%$ of mass 95 & 20.3 \\
\hline 75 & $30.0 \div 80.07$ of mass 95 & 50.7 \\
\hline 95 & Base peak, 1008 relative abundance & 100 \\
\hline 96 & $5.0-9.08$ of mass 95 & 6.7 \\
\hline 173 & Less than 2.08 of mass 174 & 0.91 \\
\hline 174 & $50.0=1208$ of mass 95 & 86.5 \\
\hline 175 & $5.0-9.08$ of mass 174 & 6.11 \\
\hline 176 & $95.0-101 \%$ of mass 174 & $84.1(97.2) 1$ \\
\hline 177 & $5.0-9.08$ of mass 176 & $5.8(6.9) 2$ \\
\hline
\end{tabular}

1 - Value is omass $174 \quad 2$ - Value is ornass 176

\begin{tabular}{|c|c|c|c|c|}
\hline $\begin{array}{c}\text { EPA } \\
\text { SAMPLE NO. }\end{array}$ & $\begin{array}{c}\text { LAB } \\
\text { SAMPLE ID }\end{array}$ & $\begin{array}{c}\text { LAB } \\
\text { FI.LE ID }\end{array}$ & $\begin{array}{c}\text { DATE } \\
\text { ANALYZED } \\
\end{array}$ & $\begin{array}{c}\text { TIME } \\
\text { ANALYZED }\end{array}$ \\
\hline VSTD0.5DI & IC $200-26335 / 5$ & DHT05.D & $10 / 06 / 2011$ & 1331 \\
\hline VSTD001DI & IC $200-26335 / 6$ & DHTO6.D & $10 / 06 / 2011$ & 1356 \\
\hline VSTD005DI & ICIS $200-26335 / 7$ & DHT07.D & $10 / 06 / 2011$ & 1421 \\
\hline VSTD010DI. & IC $200-26335 / 8$ & DHT08.D & $10 / 06 / 2011$ & 1446 \\
\hline VSTD020DI & IC $200-26335 / 9$ & DHT09.D & $10 / 06 / 2011$ & 2511 \\
\hline
\end{tabular}


$5 A$ - TORM V VOA.

VOLATLLE ORGANICS INSTRUMENT

PETREORMANCF' CHECK

BROMOFLUOROBENZENE (BFB)
EPA SAMPIE NO.

BFBDJ
Lâb Name: 'I'ESTAMERICA BURLINGTON

Lab Code: STLV Case No.: MORRIL Mod. Ref No, :

Contract: $8 F-00302$
Jub File Id: DHTAOI.D

Instrument Id: D.i

GC Column: DB-624
ID: 0.20 (mm)

\begin{tabular}{|c|c|c|}
\hline$\pi / e$ & ION ABUNDANCE CRITERIA & $\begin{array}{l}\text { REIAATTVE } \\
\text { ABUNDANCE }\end{array}$ \\
\hline 50 & $15.0-40.0$ f of mass 95 & 20.0 \\
\hline 75 & $30.0-80.0 \%$ of mass 95 & 52.1 \\
\hline 95 & Base peak, 1008 rejative abundance & 100 \\
\hline 96 & $5.0-9.0 \%$ of mass 95 & 7.0 \\
\hline 173 & Less than $2.0 \%$ of mass 174 & 0.81 \\
\hline 174 & $50.0-120 \%$ of mass 95 & 87.0 \\
\hline 17.5 & $5.0-9.0 \%$ of mass $1 \%$ & 6.21 \\
\hline 176 & $95.0-101 \%$ of mass 174 & $84.0(96.6) 1$ \\
\hline 177 & $5.0-9.08$ of mass 176 & $5.6(6.7) 2$ \\
\hline
\end{tabular}

1 - Value is omass 174

2 - Value is gmass 176

\begin{tabular}{|c|c|c|c|c|}
\hline $\begin{array}{c}\text { EPA } \\
\text { SAMPIE NO. }\end{array}$ & $\begin{array}{r}\text { LAB } \\
\text { SAMPLE ID }\end{array}$ & $\begin{array}{c}\text { LAB } \\
\text { EILE ID }\end{array}$ & $\begin{array}{c}\text { DATE } \\
\text { ANALYZED }\end{array}$ & $\begin{array}{c}\text { TIME } \\
\text { ANALYZED }\end{array}$ \\
\hline VSTD005DJ & CCVIS $200-26380 / 2$ & DHTAOZ.D & $10 / 07 / 2011$ & 0730 \\
\hline VBIJKDJ & MB $200-26380 / 3$ & DHTA03.D & $10 / 07 / 2011$ & 0754 \\
\hline $\begin{array}{l}\text { MRQCTB-W-3 } \\
0090\end{array}$ & $200-7357-5$ & DHTA04.D & $10 / 07 / 2011$ & $0 \overline{35}$ \\
\hline $\begin{array}{l}\text { MRSM2-W-30 } \\
063\end{array}$ & $2.00-7357-1$ & DHTA05.D & $10 / 07 / 2011$ & 0859 \\
\hline $\begin{array}{l}\text { MRMW2S-W-3 } \\
0069\end{array}$ & $200-7357-2$ & DHTA06.D & $10 / 07 / 2011$ & 0924 \\
\hline $\begin{array}{l}\text { MRMW SS-W-3 } \\
0071\end{array}$ & $200-7357-3$ & DH'AO $7 . \mathrm{D}$ & $10 / 07 / 2011$ & 0949 \\
\hline $\begin{array}{l}\text { MRMW 1 1S-W- } \\
30078\end{array}$ & $200-7357-4$ & DHTA08.D & $10 / 07 / 2011$ & 1014 \\
\hline VIBLKDM & VIBLK $200-26380 / 9$ & DHTA09.D & $10 / 07 / 2011$ & 1038 \\
\hline $\begin{array}{l}\text { MRMW1 1S-W- } \\
30078 \mathrm{DL}\end{array}$ & $200-1357-4$ & DHTA10.D & $10 / 07 / 2011$ & 1115 \\
\hline VHBLKO1 & $200-7357-6$ & DHTAI1.D & $10 / 07 / 2011$ & 1140 \\
\hline VSTDO05JD & CCVC $200-26380 / 25$ & DHTA25.D & $10 / 07 / 2011$ & 1844 \\
\hline
\end{tabular}


BA - FORM VIII VOA

VOLATILE INTERNAL STANDARD ARE'A RAND RETENTION TIME SUMMARY

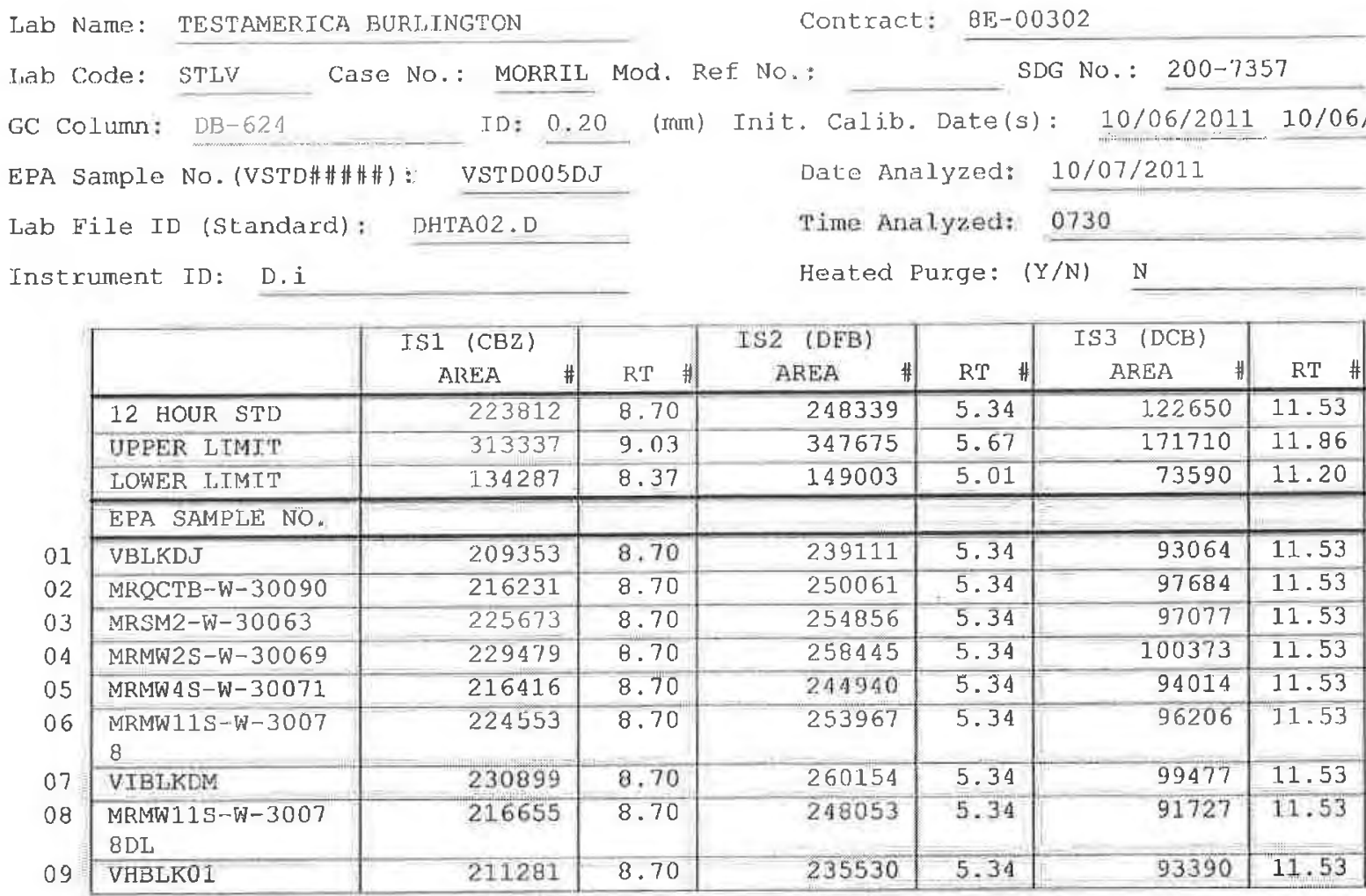

IS1 (CBZ) = Chlorobenzene-d5

IS2 $(\mathrm{DFB})=1,4-\mathrm{Difl}$.uorobenzene

IS3 $(D C B)=1,4-$ Dichlorobenzene-d4

AREA UPPER LIMIT $=140 \%$ (Trace Volatiles) of internal standard area AREA LOWER LIMIT $=60 \%$ (TIace Volatiles) of internal standard area RT UPPER LIMIT $=+0.33$ (Trace Volatiles) minutes of internal standard RT RT LOWER LIMIT $=-0.33$ (Trace Volatiles) minutes of internal standard R'T

\# Colunn used to flag values outside contract required QC liraits with an asterisk. 
$1 A$ - FORM I VOA-1

VOLA'ILE ORGANICS ANALYSIS DATA SHEET
HPA SAMPJE NO.

MRMW $11 S-W-30078$

Lab Name: TESTAMERICA BURLINGI'ON

Contract: $\quad$ 8E-00302

Lab Code: STLV Case No.: MORRTI, Mod. Ref No.: SDG No.: $200-7357$

Matrix: (SOIL/SED/WAI'ER) Water

Sample wt/vol: 25.0 (g/mL) mL

L.eve l: (IRACE/LOW/MED) IRACE

a Moisture: not dec.

GC Column: $1 \mathrm{BB}-624$

Soil Extract Volume: TD: 0.20 (mum) (uL)

Purge Voliane: 25.0 (mL)
Lab Sample ID: 200-735\%-4

Lab File ID: DHTAOB.D

Date Reccived: 10/05/2011

Date Analyzed: 10/07/2011

Dilution Factor: 1.0

Soil Aliquot Volume;

(uI)

\begin{tabular}{|c|c|c|c|}
\hline CAS 170 & COMPOUND & $\begin{array}{l}\text { CONCENTRATION UNITS: } \\
(\mathrm{ug} / \mathrm{L} \text { or } \mathrm{ug} / \mathrm{kg}) \mathrm{ug} / \mathrm{L}\end{array}$ & $Q$ \\
\hline $75-71-8$ & Dichlorodifluoromethane & 0.50 & $\mathrm{U}$ \\
\hline $79-87-3$ & Chloromethane & 0.50 & $\mathrm{U}$ \\
\hline $75-01-4$ & Vinyl chloride & 0.50 & $\mathrm{U}$ \\
\hline $74-8.3-9$ & Bromomethane & 0.50 & $\mathrm{U}$ \\
\hline $75-00-3$ & Chloroethane & 0.50 & $\mathrm{U}$ \\
\hline $75-69-4$ & Trichlorofluoromethare & 0.50 & $\mathrm{U}$ \\
\hline $75-35-4$ & 1,1-Dichloroethene & 0.50 & $\mathrm{U}$ \\
\hline $76-13-1$ & 1,1,2-Trichloro-1,2,2-trifluoroethane & 0.50 & $\mathrm{U}$ \\
\hline $67-64-1$ & Acetone & 1.1 & $\mathrm{~J} B$ \\
\hline $75-15-0$ & Carbon disulfide & 0.038 & J $B$ \\
\hline $79-20-9$ & Methyl acetate & 0.50 & $\mathrm{U}$ \\
\hline $75-09-2$ & Methylene Chloride & 0.50 & [] \\
\hline $156-60-5$ & trans-1,2-Dichloroethene & 0.50 & $\mathrm{U}$ \\
\hline $1634-04-4$ & Methyl tert-butyl ether & 0.50 & $\mathrm{U}$ \\
\hline $75-31-3$ & 1,1-Dichloroethane & 0.50 & $\mathrm{U}$ \\
\hline $156-59-2$ & cis-1,2-Dichloroethene & 0.50 & $\mathrm{U}$ \\
\hline $78-93-3$ & 2-Butanone & 5.0 & $\mathrm{U}$ \\
\hline $74-97-5$ & Bromochloromethane & 0.50 & $\mathrm{U}$ \\
\hline $67-66-3$ & Chloroform & 0.85 & \\
\hline $71-55-6$ & $1,1,1$-Trichloroethane & 0.50 & $\mathrm{U}$ \\
\hline $1.10-82-7$ & Cyclohexane & 0.50 & $\mathrm{U}$ \\
\hline $56-23-5$ & Carbon tetrachloride & 38 & $\mathrm{E} B$ \\
\hline $71-43-2$ & Benzene & 0.50 & $\mathrm{U}$ \\
\hline $107-06-2$ & 1,2-Dichloroethane & 0.50 & U \\
\hline
\end{tabular}

Report 1,4-Dioxane for t.ow-Medium VoA analysis only 
$1 B$ - FORM I VOA-2

VOLAPILE ORGANICS ANALYSIS DATA SHEET
EPA SAMPLE NO.

MRMW11.3-W-30078

Lab Name: TESTAMERICA BURLINGTON

Contract: $\quad 8 E-00302$

Lab Code: STLV Case No.: MORRIJ, Mod, Ref No.:

SDG No.: $\quad 200-7357$

Matrix: (SOIL/SED/WATER) water

Sample wt/vol: $25.0 \quad$ (g/mL) $\mathrm{mL}$

Level: (TRACE/LOW/MED) TRACE

o Moisture: not dec.

GC Columi: DB-624

Soil Extract Volume: ID: $0.20 \quad(\mathrm{~mm})$

Purge Volume: 25.0 (uL)

\section{(m山)}

Lab Sample ID: 200 $\quad$-7357-4

Lab File ID: DHTA08.D

Date Received: 10/05/2011

Date Analyzed: 10/07/2011

Dilution Factor: 1.0

Soil Aliquot Volumes

(UL)

\begin{tabular}{|c|c|c|c|}
\hline CAS NO, & COMPOUND & $\begin{array}{l}\text { CONCENTRATION UNITS: } \\
(\mathrm{ug} / \mathrm{L} \text { or } \mathrm{ug} / \mathrm{kg}) \mathrm{ug} / \mathrm{L}\end{array}$ & $Q$ \\
\hline $79-01-6$ & Trichloroethene & 0.50 & $\mathrm{U}$ \\
\hline $108-87-2$ & Methylcyclohexane & 0.50 & $\mathbf{U}$ \\
\hline $78-87-5$ & 1;2-Dichloropropanë & 0.50 & $\mathrm{U}$ \\
\hline $75-27-4$ & Bromodichloromethane & 0.50 & $\mathrm{U}$ \\
\hline $10061-01-5$ & cis-1,3-Dj.ch.loropropene & 0.50 & $\mathrm{U}$ \\
\hline $108-10-1$ & 4-Methyl-2-pentanone & 5.0 & $\mathrm{U}$ \\
\hline $108-86-3$ & Toluene & 0.024 & J B \\
\hline $10061-02-6$ & trans $-1,3 \cdots$ Dichloropropene & 0.50 & U \\
\hline $79-00-5$ & 1, 1,2-Trichloroethane & 0.50 & $\mathrm{U}$ \\
\hline $127-18-4$ & Tetrachloroethene & 0.50 & $\mathrm{U}$ \\
\hline $591-78-6$ & 2-Hexanone & 5.0 & $\mathrm{U}$ \\
\hline $124-48-1$ & Dibromoch loromethane & 0.50 & $\mathrm{U}$ \\
\hline $106-93-4$ & 1,2-Dibromoethane & 0.50 & $\mathrm{U}$ \\
\hline $10 \theta-90-7$ & Chlorobenzene & 0.50 & $\mathrm{U}$ \\
\hline $100-41-4$ & Ethylbenzene & 0.50 & $\mathrm{U}$ \\
\hline $95-47-6$ & o-xylene & 0.50 & $\mathrm{U}$ \\
\hline $17 \overline{9601-2 \overline{3}-1}$ & $\mathrm{~m}, \mathrm{p}-\mathrm{xylene}$ & 0.0082 & $\mathrm{~J} \mathrm{~B}$ \\
\hline $100-42-5$ & Styrene & 0.50 & U \\
\hline $75-25-2$ & Bromoform & 0.50 & $\mathrm{U}$ \\
\hline $98-82-8$ & IsopropyJbenzene & 0.50 & $\mathrm{U}$ \\
\hline $79-34-5$ & $1,1,2,2$-Tetrachloroethane & 0.50 & $\mathrm{U}$ \\
\hline $541-73-1$ & 1,3-Dichlorobenzene & 0.50 & $\mathrm{U}$ \\
\hline $106-46-7$ & 1,4-Dichlorobenzene & 0.50 & $\mathrm{U}$ \\
\hline $95-50-1$ & 1,2-Dichlorobenzene & 0.50 & $\mathrm{U}$ \\
\hline $96-12-8$ & 1,2-Dibromo-3-chloropropane & 0.50 & U] \\
\hline $120-82-1$ & $1,2,4$-Trichlorobenzene & 0.50 & U \\
\hline $87-61-6$ & $1,2,3$-Trichlorobenzene & 0.50 & $\mathrm{U}$ \\
\hline
\end{tabular}


$1 \mathrm{~J}$ - FORM I VOA-TIC

VOLATILE ORGANICS ANALYSIS DATA SHEET TENTATIVELY IDENTIEIED COMPOUNDS
EPA SAMPLE NO.

MRMW11S-W-30078

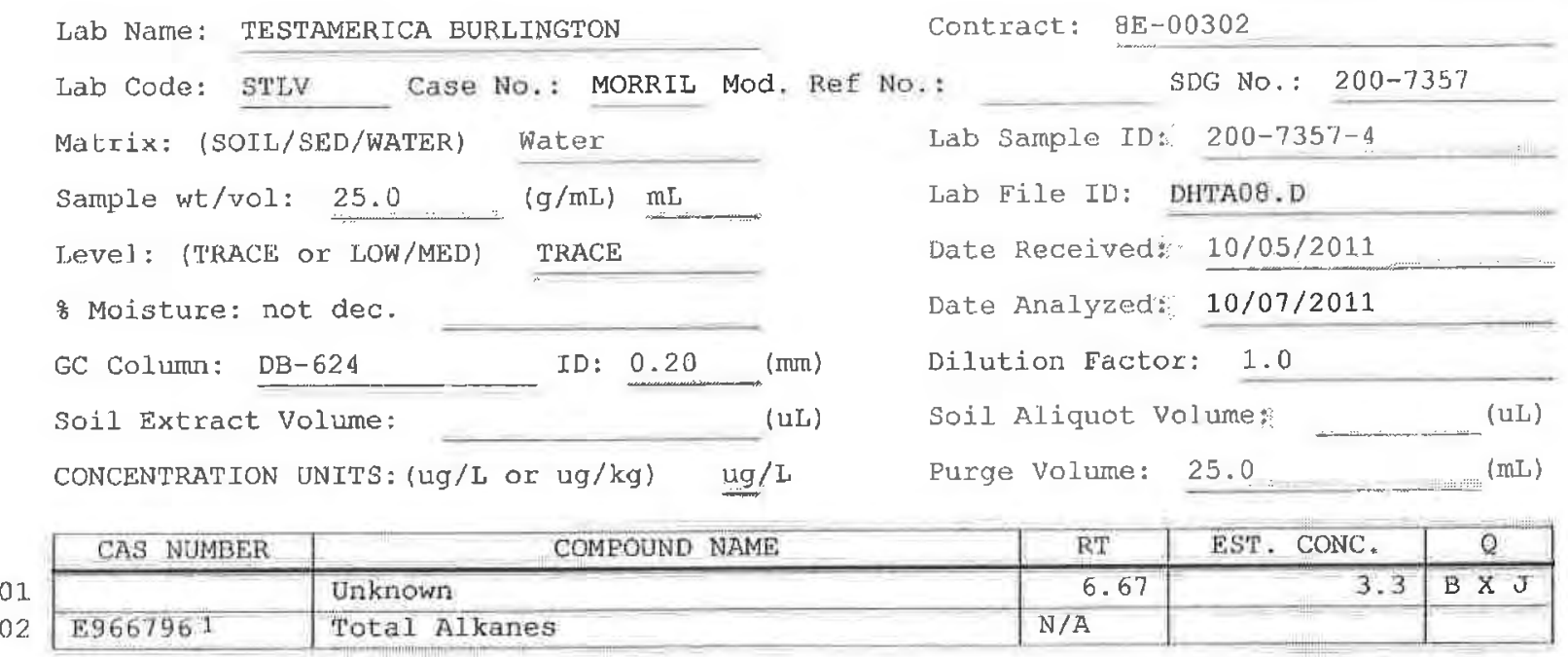

1 EPA-designated Registry Number. 
$1 A$ - FORM I VOA-1

VOLATILE ORGANICS ANALYSIS DAIA SHEET
EPA SAMPLE NO.

MRMW11S-W-30078DL
Lab Name: TESTPMERICA BURLINGTON

Lab Code: STIV

Matrix: (SOIL/SED/WATER)

Sarnple wt/vol: 25.0

Level: ('RACE/LOW/MED)

TRACE

Moisture: not dec.

GC Col.urnn: DB-624

Soil Txtract Volume:

ID: 0.20

Purge Volume: 25.0
Contract: $8 \mathrm{E}-00302$

SDG NO.: 200-7357

\begin{tabular}{|c|c|c|c|}
\hline CAS NO. & COMPOUND & $\begin{array}{l}\text { CONCENTRATION UNITS: } \\
(\mathrm{ug} / \mathrm{L} \text { or } \mathrm{ug} / \mathrm{kg}) \mathrm{ug} / \mathrm{L}\end{array}$ & $Q$ \\
\hline $75-71-8$ & Dichlorodifluorornethane & 1.1 & $\mathrm{U}$ \\
\hline $74-87-3$ & Chloromethane & 1.1 & $\mathrm{U}$ \\
\hline $75-01-4$ & Vinyl chloride & 1.1 & $\mathrm{U}$ \\
\hline $74-83-9$ & Bromomethane & 1.1 & $\mathrm{U}$ \\
\hline $75-00-3$ & Chloroethane & 1.1 & $\mathrm{U}$ \\
\hline $75-69-4$ & Trichlorofluoromethane & 1.1 & $\mathrm{U}$ \\
\hline $75-35-4$ & 1,1-Dichloroethene & 1.1 & $\mathrm{U}$ \\
\hline $76-13-1$ & 1,1,2-Trichloro-1,2,2-trifluoroethane & 1.1 & $\mathrm{U}$ \\
\hline $67-64-1$ & Acetone & 1.1 & $\mathrm{U}$ \\
\hline $75-15-0$ & Carbon disulfide & 0.078 & $\mathrm{~J} \mathrm{D} \mathrm{B}$ \\
\hline $79-20-9$ & Methyl acetate & 1.1 & U \\
\hline $75-09-2$ & Methylene Chloride & 1.1 & U \\
\hline $156-60-5$ & trans-1,2-Dichloroethene & 1.1 & $\mathrm{U}$ \\
\hline $1634-04-4$ & MethyI tert-butyl ether & 1.1 & $\mathrm{U}$ \\
\hline $75-34-3$ & 1,1 -Dichloroethane & 1.1 & $\mathrm{U}$ \\
\hline $156-59-2$ & cis-1,2-Dichloroetnene & 1.1 & 0 \\
\hline $78-93-3$ & 2.-Butanone & 11 & $\mathrm{U}$ \\
\hline $74-97-5$ & Bromoch loromethane & 1.1 & $\mathrm{U}$ \\
\hline $67-66-3$ & Chloroform & 0.84 & $\mathrm{~J} \mathrm{D}$ \\
\hline $71-55-6$ & 1,1,1-Trichloroethane & 1.1 & U \\
\hline $110-82-7$ & Cyclohexane & 1.1 & $\mathrm{U}$ \\
\hline $56-23-5$ & Carbon tetrachloxide & 35 & D B \\
\hline $71-43-2$ & Benzene & 1.1 & $\mathrm{U}$ \\
\hline $107-06-2$ & 1,2-Dichloroethane & 1.1 & $\mathrm{U}$ \\
\hline
\end{tabular}

Report 1,4-Dioxane for Low-Medium VOA analysis only 
$1 \mathrm{~B}$ - FORM I VOA-2

VOLATILE ORGANICS ANALYSIS DATA SHEET
EPA SAMPLF NO.

MRMW11S-W-30078DL
Lab Name: 'I'ES'TAMERICA BURLING'TON

Lab Code: STLV

Case No.: MORRIL Mod. Ref No.:
Contract: $8 E-00302$

SDG No.: $200-7357$
Matrix: (SOIL/SED/WATER) $\frac{\text { Water }}{\text { (g/mL) } \mathrm{mL}}$
Sample wt/vol: 25.0
Level: (TRACE/LOW/MED) TRACE
of Moisture: not dec.
GC Column: DB-624 ID: 0.20 (mm)
Soil Extract Volume:

purge Volume: 25.0
(mL)
Jab Sample ID: $200-7357-4$

Lab File ID: DHTA10.D

Date Received: 10/05/2011

Date Analyzed: 10/07/2011

Dilution Factor: 2.2

Soil Aliquot Volume:

\begin{tabular}{|c|c|c|c|}
\hline CAS NO. & COMPOUND & $\begin{array}{l}\text { CONCENTRATION UNITS: } \\
(\mathrm{ug} / \mathrm{L} \text { or } \mathrm{ug} / \mathrm{kg}) \mathrm{ug} / \mathrm{L}\end{array}$ & $Q$ \\
\hline $79-01-6$ & 'Trichloroethene & 1.1 & $\mathrm{U}$ \\
\hline $108-87-2$ & Methylcyclohexane & 1.1 & U \\
\hline $78-87-5$ & 1,2-Dichloropropane & 1.1 & $\mathrm{U}$ \\
\hline $75-27-4$ & Bromodichloromethane & 1.1 & $U$ \\
\hline $10061-01-5$ & cis-1,3-Dichloropropene & 1.1 & $\mathrm{U}$ \\
\hline $108-10-1$ & 4-Methyl-2-pentanone & 11 & $U$ \\
\hline $108-88-3$ & Toluene & 0.033 & J D B \\
\hline $10061-02-6$ & trans-1,3-Dichloropropene & 1.1 & $\mathrm{U}$ \\
\hline $79-00-5$ & 1. 1,2-Trichloroethane & 1.1 & U \\
\hline $127-18-4$ & Tetrachloroethene & 1.1 & U \\
\hline $591-78-6$ & 2-Hexanone & 11 & U \\
\hline $124-48-1$ & Dibromochloromethane & $1 \cdot 1$ & $\mathrm{U}$ \\
\hline $.206-93-4$ & 1,2-Dibromoethane & 1.1 & $\mathrm{U}$ \\
\hline $108-90-7$ & Chlorobenzene & 1.1 & $\mathrm{U}$ \\
\hline $100-41-4$ & Ethylbenzene & 1.1 & U \\
\hline $95-47-6$ & o-xylene & 1.1 & $\mathrm{U}$ \\
\hline $179601-23-1$ & $m, p-x y l e n e$ & 0.020 & J D B \\
\hline $100-42-5$ & Styrene & 1.1 & U \\
\hline $75-25-2$ & Bromoform & 1.1 & $\mathrm{U}$ \\
\hline $98-82-8$ & Isopropylbenzene & 1.1 & $\mathrm{U}$ \\
\hline $79-34-5$ & $1,1,2,2$-Tetsachloroethane & 1.1 & $\mathrm{U}$ \\
\hline $541-73-1$ & 1,3-Dichloraberzene & 1.1 & $\mathrm{U}$ \\
\hline $106-46-7$ & 1,4-Dichlorobenzene & 1.1 & ए \\
\hline $95-50-1$ & 1,2-Dichlorobenzene & 1.1 & $\mathrm{U}$ \\
\hline $96-12-8$ & 1,2-Dibromo-3-Chloropropane & $1 \cdot 1$ & $\mathrm{U}$ \\
\hline $120-82-1$ & $1,2,4$-I'richlorobenzene & 1.1 & $\mathrm{U}$ \\
\hline $87-6 i-6$ & $1,2,3$-Trichlorobenzene & 1.1 & U \\
\hline
\end{tabular}


1J - FORM I VOA-TIC

VOLATILE ORGANICS ANALYSIS DATA SHEET TENTATIVELY IDENTIFIED COMPOUNDS
EPA SAMPLE NO.

MRMW11S-W-30078DL

Lab Name: TESTAMERICA BURLINGTON

Contract: $8 \mathrm{E}-00302$

Lab Code: STLV Case No.: MORRIL Mod. Ref No.:

SDG No.: 200-7357

Matrix: (SOIL/SED/WATER) Water

Sample wt/vol: 25.0 $(\mathrm{g} / \mathrm{mJ}) \quad \mathrm{mL}$

Leve1: (TRACE or LOW/MED)

TRACE

\% Moisture: not dec.

GC Column: DB-624 ID: $0.20 \quad$ (mm)

Soil Extract Volume: (uL)

CONCENTRATION UNITS: (ug/L Or $\mathrm{ug} / \mathrm{kg}$ ) $\mathrm{ug} / \mathrm{L}$
Lab Sample ID: 200-7357-4

Lab File ID: DHTA10.D

Date Received: 10/05/2011

Date Analyzed 10/07/2011

Dilution Factor: 2.2

Soil Aliquot Volume:

Purge Volume: 25.0 (mL)

\begin{tabular}{|l|l|l|c|c|c|}
\hline \multicolumn{1}{|c|}{ CAS NUMBER } & \multicolumn{1}{|c|}{ COMPOUND NAME } & RT & EST. CONC. & $Q$ \\
\cline { 2 - 6 } 01 & & 6.67 & & 6.4 & $\begin{array}{l}\text { B X D } \\
\text { J }\end{array}$ \\
\hline
\end{tabular}

1 ЕРA-designated Registry number. 
$1 A$ - FORM I VOA-1

VOLATILE ORGANICS ANALYSIS DATA SHEET
EPA SAMPLE NO.

MRMW2S-W-30069

Laל Name: T'ESTAMERICA BURLINGTON

Contract: $\quad 8 \mathrm{E}-00302$

Lab Code: STLV Case No.: MORRIL Mod. Ref No.:

SDG No.: 200-7357

Matrix: (SOIL/SED/WATER) Water

Sample wt/vol: 25.0

$(\mathrm{g} / \mathrm{mL}) \mathrm{mL}$

Level: ('TRACE/LOW/MED)

TRACE

$\&$ Moisture: not dec.

GC Column: DB-624

ID: $0.20 \quad$ (mm)

Soil Extract Volume: (1.L)

Purge Volume: 25.0

(mL)
Lab Sample ID: 200-7357-2

Lab File ID: DHTA06.D

Date Recelved: 10/05/2011

Date Analyzed: 10/07/2011

Dilution Factor: 1.0

Soil Aliquot volume:

(uL)

\begin{tabular}{|c|c|c|c|}
\hline CAS NO. & COMPOUND & $\begin{array}{l}\text { CONCENTRATION JUNITS: } \\
(\mathrm{ug} / \mathrm{L} \text { or } \mathrm{ug} / \mathrm{kg}) \mathrm{ug} / \mathrm{L}\end{array}$ & Q \\
\hline $75-71-8$ & Dichlorodifluoromethane & 0.50 & $\overline{\mathrm{U}}$ \\
\hline $74-87-3$ & Chloromethane & 0.50 & U \\
\hline $75-01-4$ & Vinyl chloride & 0.50 & $\mathrm{u}$ \\
\hline $74-83-9$ & Bromomethane & 0.50 & u \\
\hline $75-00-3$ & Chloroethane & 0.50 & $\mathrm{U}$ \\
\hline $75-69-4$ & Trichlorofluoromethane & 0.50 & U \\
\hline $75-35-4$ & 1,1-Dichloroethene & 0.50 & $\mathrm{U}$ \\
\hline $76-13-1$ & $1,1,2$-Trichloro-1,2,2-trifluoroethane & 0.50 & 0 \\
\hline $67-64-1$ & Acetone & 5.0 & U \\
\hline $75-15-0$ & Carbon disulfide & 0.036 & $\mathrm{IB}$ \\
\hline $79-20-9$ & Methyl acetate & 0.50 & U \\
\hline $75-09-2$ & Methylene Chloride & 0.50 & $\mathrm{U}$ \\
\hline $156-60-5$ & trans-1,2-Dichloroethene & 0.50 & $\mathrm{U}$ \\
\hline $1634-04-4$ & Methyl tert-butyl ether & 0.50 & $\mathrm{U}$ \\
\hline $75-34-3$ & 1,1-Dichloroethane & 0.50 & 0 \\
\hline $156-59-2$ & cj.s-1,2-Dichloroethene & 0.50 & 0 \\
\hline $78-93-3$ & 2-Butanone & 5.0 & $\mathrm{u}$ \\
\hline $74-97-5$ & Bromochloromethane & 0.50 & U \\
\hline $67-66-3$ & Chloroform & 0.50 & $u$ \\
\hline $71-55-6$ & 1, 1,1-Trichloroethane & 0.50 & 0 \\
\hline $110-82-7$ & Cyclohexane & 0.50 & $\mathrm{u}$ \\
\hline $56-23-5$ & Carbon tetrachloride & 0.043 & J B \\
\hline $71-43-2$ & Benzene & 0.50 & $\mathrm{u}$ \\
\hline $107-06-2$ & 1,2-Dichloroethane & 0.50 & $\mathrm{U}$ \\
\hline
\end{tabular}

Report 1,4-Dioxane for Low-Medium VOA analysis onì 
Lab Name: TESTAMERICA BURLINGTON

Contract: $\quad 8 \mathrm{E}-00302$

Iab Code: STLV Case No.: MORRIL Mod. Ref No.: SDG No.: $200-7357$

Matrix: (SOTL/SED/WRTER) Water

Sample wt/vol: 25,0 $(\mathrm{g} / \mathrm{mL}) \mathrm{mL}$

Leve 1: (TRACE/LOW/MED) TRACE

8 Moisture: not dec.

GC Column: DB-624

ID : 0.20 (man)

Soil Extract Volume:

(uL)

Purge Volume: 25.0 (mL)
Lab Sample $\overline{\mathrm{i}} \mathrm{D}: \quad 200-7357-2$

Tab File ID: DHTA06.D

Date Received: 10/05/2011

Date Analyzed: 10/07/2011

Dilution Factor: 1.0

Soil Aliquot Volme:

(uL)

\begin{tabular}{|c|c|c|c|}
\hline CAS NO. & COMPOUND & $\begin{array}{l}\text { CONCENTRATION UNITS: } \\
(\mathrm{ug} / \mathrm{L} \text { or } u \mathrm{~g} / \mathrm{kg} \text { ) ug/L }\end{array}$ & Q \\
\hline $79-01-6$ & Trichloroethene & 0.50 & U \\
\hline $108-87-2$ & Methyleyclohexane & 0.50 & U \\
\hline $78-87-5$ & 1,2-Dicliloropropane & 0.50 & U \\
\hline $75-27-4$ & Bromodichloromethane & 0.50 & U \\
\hline $10061-01-5$ & cis-1,3-Dichloropropene & 0.50 & $\mathrm{U}$ \\
\hline $108-10-1$ & 4-Methyl-2-pentanone & 5.0 & $\mathrm{U}$ \\
\hline $108-88-3$ & T'oluene & 0.025 & $\mathrm{~J} \mathrm{~B}$ \\
\hline $10061-02-6$ & trans-1,3-Dichloropropene & 0.50 & $\mathrm{U}$ \\
\hline $79-00-5$ & 1,1,2-Trichloroethane & 0.50 & $\underline{\mathrm{U}}$ \\
\hline $127-18-4$ & Tetrachloroethene & 0.50 & [] \\
\hline $591-78-6$ & 2 -Hexanone & 5.0 & U \\
\hline $124-48-1$ & Dibromoch loromethane & 0.50 & $\mathrm{U}$ \\
\hline $106-93-4$ & 1,2-Dibromoethane & 0.50 & $\mathrm{U}$ \\
\hline $108-90-7$ & Chlorobenzene & 0.50 & $\mathrm{U}$ \\
\hline $100-41-4$ & Ethylberzene & 0.50 & $\mathrm{U}$ \\
\hline $95-47-6$ & o-Xylene & 0.50 & [] \\
\hline $179601-23-1$ & m, p-xylene & 0.021 & $\mathrm{~J} B$ \\
\hline $100-42-5$ & Slyyene & 0.50 & $\mathrm{U}$ \\
\hline $75-25-2$ & Bromoform & 0.50 & 0 \\
\hline $98-82-8$ & Isopropylbenzene & 0.50 & U \\
\hline $79-34-5$ & $1,1,2,2$-Tetrachloroethane & 0.50 & $\mathrm{U}$ \\
\hline $541-73-1$ & 1,3-Dichlorobenzene & 0.50 & $\mathrm{U}$ \\
\hline $106-46-7$ & 1,4-Dichlorobenzene & 0.50 & $\mathrm{U}$ \\
\hline $95-50-1$ & 1,2-Dichlorobenzene & 0.50 & $\mathrm{U}$ \\
\hline $96-12-8$ & 1,2-Dibromo-3-Chloropropane & 0.50 & $\mathrm{U}$ \\
\hline $120-82-1$ & 1,2,4-'Trichlorobenzene & 0.50 & $\mathrm{U}$ \\
\hline $87-61-6$ & 1,2,3-Trichlorobenzene & 0.50 & U \\
\hline
\end{tabular}


$1 \mathrm{~J}$ - FORM I VOA-TIC

VOLATILE ORGANICS ANALYSIS DATA SHEET TENTATIVELY IDENTIFIED COMPOUNDS
EPA SAMPLE NO.

MRMW2S-W-30069

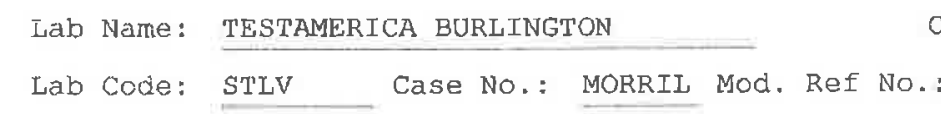

Matrix: (SOII/SED/WATER) Water

Sample wt/vol: $25.0 \ldots(\mathrm{g} / \mathrm{mL}) \mathrm{mI}$

Ievel: (TRACE or LOW/MED) TRACE

o Moisture: not dec.

GC Column: DB-624

Soil Extract Volume: ID: $0.20 \quad(\mathrm{~mm})$

CONCENTRATION UNI'IS: (ug/L or $\mathrm{ug} / \mathrm{kg}$ ) ug/L
Contract: $\quad 8 E-00302$

SDG No.: 200-7357

I.ab Sample ID: 200-7357-2

Lab File ID: DHTA06.D

Date Received: 10/05/2011

Date Analyzed: 10/07/2011

Dilution Factor: 1.0

Soil Aliquot Volume:

(uI)

Purge Volume: 25.0 (mL)

\begin{tabular}{|l|l|l|r|r|r|r|r|}
\hline \multicolumn{1}{|c|}{ CAS NUMBER } & COMPOUND NAME & RT & EST. CONC. & Q \\
\hline
\end{tabular}

IEPA-designated Registry Number. 
$1 A$ - FORM I VOA-1

VOLATILE ORGANICS ANALYSIS DATA SHEE'
EPA SAMPLE NO.

MRMW4S-W-3007
Lab Name: TESTAMERICA BURLINGTON

Lab Code: STLV $\mathrm{Ca}$

Matrix: (SOIL/SED/WATER)

water

Sample wt/vol: 25.0 $(\mathrm{g} / \mathrm{mL}) \mathrm{mL}$

Leve]: (TRACE/LOW/MED) TRACE

\& Moisture: not dec.

GC Colurn: DB-624

ID: 0.20 (Im)

Soil Extract Volume:

Purge Volume: 25.0

(mL)
Contract: $\quad$ BE-00302

SDG No.: 200-7357

\begin{tabular}{|c|c|c|c|}
\hline CAS NO: & COMPOUND & $\begin{array}{l}\text { CONCENTRATION UNITS: } \\
(\mathrm{ug} / \mathrm{L} \text { or } \mathrm{ug} / \mathrm{kg}) \mathrm{ug} / \mathrm{L}\end{array}$ & $Q$ \\
\hline $75-71-8$ & Dichlorodifluoromethane & 0.50 & $\mathrm{U}$ \\
\hline $74-87-3$ & Chloromethane & 0.50 & U \\
\hline $75-01 \cdots 4$ & Viny1 chloride & 0.50 & U \\
\hline $74-83-9$ & Bromomethane & 0.50 & U \\
\hline $75-00-3$ & Chloroethane & 0.50 & $\mathrm{U}$ \\
\hline $75-69-4$ & Trichlorofluoromethane & 0.50 & $\mathrm{U}$ \\
\hline $75-35-4$ & 1,1-Dichloroethene & 0.50 & $\mathrm{U}$ \\
\hline $76-13-1$ & 1,1,2-Trichloro-1,2,2-trifluoroethane & 0.50 & 0 \\
\hline $67-64-1$ & Acetone & 0.98 & $\mathrm{~J} \mathrm{~B}$ \\
\hline $75-15-0$ & Carbon disulfide & 0.037 & $\mathrm{~J} B$ \\
\hline $79-20-9$ & Methyl acetate & 0.50 & U \\
\hline $75-09-2$ & Methylene Chloride & 0.50 & U \\
\hline $156-60-5$ & trans-1,2-Dichloroethene & 0.50 & $\mathrm{U}$ \\
\hline $1634-04-4$ & Methyl tert-butyl ether & 0.50 & U \\
\hline $75-34-3$ & 1,1-Dichloroethane & 0.50 & 0 \\
\hline $156-59-2$ & cis-1,2-Dichloroethene & 0.50 & $\mathrm{U}$ \\
\hline $78-93-3$ & 2-Butanone & 5.0 & $\mathrm{U}$ \\
\hline $74-97-5$ & Bromochloromethane & 0.50 & U \\
\hline $67-66-3$ & Chlorcform & 0.50 & $\mathrm{u}$ \\
\hline $71-55-6$ & 1,1,1-Trichloroethane & 0.50 & 0 \\
\hline $110-82-7$ & Cyclohexane & 0.50 & u \\
\hline $56-23-5$ & Carbon tetrachloride & 0.11 & $\mathrm{JE}$ \\
\hline $71-43-2$ & Benzene & 0.50 & $\mathrm{U}$ \\
\hline $107-06-2$ & 1,2-Dichloroethane & 0.50 & 0 \\
\hline
\end{tabular}

Report 1,4-Dioxane for Low-Medium VOA analysis only

Lab Sample ID: 200-7357-3

Lab File I.D: DHTA07.D

Date Recelved: 10/05/2011

Date Analyzed: 10/07/2011

Dilution Factor: 1.0

Soil Aliquot Volume:

(uL) 
Lāb Nante: TESTAMERICA BURLINGTON

Lab Coce: STLV Case No.: MORRIL Mod. Ref No.:

Contract: $\quad 8 \mathrm{E}-00302$

Matrix: (SOIL/SED/WATER) Water

Sample wt/vol: 25.0 $(g / m L) \quad m L$

Level: (TRACE/LOW/MED) TRACE

z Moisture: not dec.

GC Column: $\quad$ DB-624

Soil Extract Volume:

Purge volume: 25.0
ID: 0.20 (InII)

(u.t.)

(mL)

SDG No.: 200-7357

Lab Sample ID: $\quad 200-7357-3$

Lab File TD: DHTA07.D

Date Received: 10/05/2011

Date Analyzed: 10/07/2011

Dilution factor: 1.0

Soil Nliquot Volume:

\begin{tabular}{|c|c|c|c|}
\hline CAS NO. & COMPOUND & $\begin{array}{l}\text { CONCENTRATION UNITS: } \\
(\mathrm{ug} / \mathrm{L} \text { or } \mathrm{ug} / \mathrm{kg}) \mathrm{ug} / \mathrm{L}\end{array}$ & $Q$ \\
\hline $79-01-6$ & Trichloroethene & 0.50 & U \\
\hline $108-87-2$ & Methylcyclohexane & 0.50 & $\mathrm{U}$ \\
\hline $78-87-5$ & 1,2-Dichloropropane & 0.50 & $\mathrm{U}$ \\
\hline $75-27-4$ & Bromodichloromethane & 0.50 & [] \\
\hline $10061-01-5$ & cis-1,3-Dichloropropene & 0.50 & $\mathrm{U}$ \\
\hline $108-10-1$ & 4-Methyl-2-pentanone & 5.0 & $\mathrm{U}$ \\
\hline $108-88-3$ & Toluene & 0.013 & $\mathrm{~J} B$ \\
\hline $10061-02-6$ & trans-1,3-Dichloropropene & 0.50 & U \\
\hline $79-00-5$ & $1,1,2$-Trichloroethane & 0.50 & $\mathrm{U}$ \\
\hline $127-18-4$ & Tetrachloroethene & 0.50 & $\mathrm{U}$ \\
\hline $591-78-6$ & 2-Hexanone & 5.0 & $\mathrm{U}$ \\
\hline $124-48-1$ & Dibromochloromethane & 0.50 & $\mathrm{U}$ \\
\hline $106-93-4$ & 1,2-Dibromoethane & 0.50 & $\mathrm{U}$ \\
\hline $108-90-7$ & Chlorobenzene & 0.50 & $\mathrm{U}$ \\
\hline $100-41-1$ & Ethy]benzene & 0.50 & $\mathrm{U}$ \\
\hline $95-47-6$ & o-Xylene & 0.50 & $\mathrm{U}$ \\
\hline $179601-23-1$ & m,p-Xylene & 0.0052 & $\mathrm{~J} B$ \\
\hline $100-42-5$ & Styrene & 0.50 & $\mathrm{U}$ \\
\hline $75-2.5-2$ & Bromoform & 0.50 & $\mathrm{U}$ \\
\hline $98-82-8$ & Isopropylbenzene & 0.50 & $\mathrm{U}$ \\
\hline $79-34-5$ & $1,1,2,2$-Tetrachloroethane & 0.50 & $\mathrm{U}$ \\
\hline $541-73-1$ & 1,3-Dichlorobenzene & 0.50 & U \\
\hline $106-46-7$ & 1,4-Dichlorobenzene & 0.50 & $\mathrm{U}$ \\
\hline $95-50-1$ & 1,2-Dichlorobenzene & 0.50 & $\mathrm{U}$ \\
\hline $96-12-8$ & 1.2-Dibromo-3-Chloropropane & 0.50 & $\mathrm{U}$ \\
\hline $120-82-1$ & 1,2,4-Trichlorobenzent & 0.50 & $\mathrm{U}$ \\
\hline $97-61-6$ & $1,2,3$-Trichlorobenzene & 0.50 & U \\
\hline
\end{tabular}


$1 \mathrm{~J} \mathrm{-} \mathrm{FORM} \mathrm{T} \mathrm{VOA-TIC}$

VOLATILE ORGANICS ANALYSIS DATA SHEET

TENTATIVELY IDENTIEIED COMPOUNDS
EPA SAMPLE NO.

MRMW 4S-W-30071

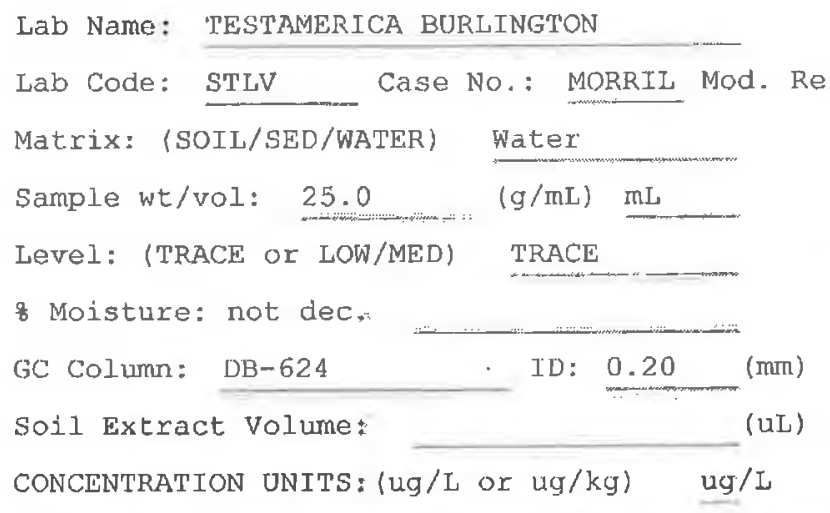

Contract: $8 \mathrm{E}-00302$

SDG No.: $200-7357$

Lab Sample ID: 200-7357-3

Lab File ID: DHTA07.D

Date Received: $10 / 05 / 2011$

Date Mnalyzed 10/07/2011

Dilution F'actor: 1.0

Soil Aliquot volume:

(uL)

Purge Volume: 25.0

(IIL)

\begin{tabular}{l|l|l|c|c|c|}
\hline \multicolumn{1}{|c|}{ CAS NUMBER } & \multicolumn{1}{|c|}{ COMPOUND NAME } & R'T & EST. CONC. & $R$ \\
\hline & & Unknown & 6.67 & 3.3 & $B \times \mathrm{J}$ \\
\hline & E9667961 & Total Alkanes & N/A & & \\
\hline
\end{tabular}

I EPA-designated Registry Number. 
$1 \mathrm{~A}$ - FORM I VOA-1

VOLATILE ORGANICS ANAI,YSIS DATA SHEE'T
EPA SAMPLE NO,

MRQCTB-W-30090

Lab Name: TESTAMERICA BURLINGTON

Contract: $\quad 8 \mathrm{~F}-00302$

Lab Code: STLV Case No.: MORRIL Mod. Ref No.:

SDG Na.: $200-7357$

Matrix: (SOIL/SED/WATER) Water

Sample wt/vol: $25.0 \ldots,(\mathrm{g} / \mathrm{mL}) \mathrm{mL} \ldots$

Level: (TRACE/LOW/MED) TRACE

음 Moisture: not dec.

GC Co.lumn: DB-624

Soil Extract Volume: ID: $0.20 \quad$ (mm)

Purge Volume: 25.0
(uI)

$\left(\mathrm{nLI} \mathrm{I}_{1}\right.$ )
Lab sample rD: 200-7357-5

Lab File ID: DHTA04.D

Date Received: $10 / 05 / 2011$

Date Analyzed: 10/07/2011

Dilution Eactor: 1.0

\begin{tabular}{|c|c|c|c|}
\hline CAS NO. & COMPOUND & $\begin{array}{l}\text { CONCENTRATION UNITS: } \\
\text { (ug/L or } \mathrm{ug} / \mathrm{kg} \text { ) ug/L }\end{array}$ & $Q$ \\
\hline $75-71-8$ & Dichlorodifluoromethane & 0.50 & $\mathbf{U}$ \\
\hline $74-87-3$ & Chloromethane & 0.50 & $\mathrm{U}$ \\
\hline $75-01-4$ & Vinyl chloride & 0.50 & $\mathrm{U}$ \\
\hline $74-83-9$ & Bromomethane & 0.50 & U \\
\hline $75-00-3$ & Chloroethane & 0.50 & U \\
\hline $75-69-4$ & Trichlorofluoromethane & 0.50 & $\mathrm{U}$ \\
\hline $75-35-4$ & 1,1-Dichloroethene & 0.50 & $\mathbf{U}$ \\
\hline $76-13-1$ & 1,1,2-Trichloro-1,2,2-trifluoroethane & 0.50 & $\mathrm{U}$ \\
\hline $67-64-1$ & Acetone & 2.5 & J B \\
\hline $75-15-0$ & Carbon disulfide & 0.065 & $\mathrm{~J} \mathrm{~B}$ \\
\hline $79-20-9$ & Methyl acetate & 0.50 & $\mathrm{U}$ \\
\hline $7.5-09-2$ & Methylene Chloride & 0.017 & $\mathrm{JB}$ \\
\hline $156-60-5$ & trans-1,2-Dichloroethene & 0.50 & $\mathrm{U}$ \\
\hline $1634-04-4$ & Methyl tert-but:yl ether & 0.50 & $\mathrm{U}$ \\
\hline $75-34-3$ & 1,1-Dichloroethane & 0.50 & $\mathrm{0}$ \\
\hline $156-59-2$ & cis-1,2-Dichloroethene & 0.50 & $\mathrm{U}$ \\
\hline $78-93-3$ & 2-Butanone & 5.0 & $\mathrm{U}$ \\
\hline $74-97-5$ & Bromochloromethane & 0.50 & $\mathrm{U}$ \\
\hline $67-66-3$ & Chioroform & 0.045 & $\mathrm{~J}$ \\
\hline $71-55-6$ & 1,1,1-Trichloroethane & 0.50 & $\mathrm{U}$ \\
\hline $110-82-7$ & Cyclohexane & 0.50 & $\mathrm{U}$ \\
\hline $56-23-5$ & Carbon tetrachloride & 0.016 & J $\mathrm{B}$ \\
\hline $71-43-2$ & Benzene & 0.050 & J \\
\hline $107-06-2$ & 1,2-Dichloroethane & 0.50 & $\mathrm{U}$ \\
\hline
\end{tabular}

Report 1,4-Dioxane for Low-Medium VOA analysis only 
$1 B$ - FORM I VOA-2

VOLA'TITH ORGANICS ANAI,YSTS DATA SHEET
EPA SAMPIJE NO.

MRQCTB-W-30090

Lab Name: 'IESTMMERICA BURLINGTON

Contract: $\quad 8 E-00302$

Lab Code: STLV Case No.: MORRIL Mod. Ref No.:

SDG NO.: 200-7357

Matrix: (SOIJ/SED/WA'TER) Water

Sample wt/vol: 25.0

$(g / m L) \quad m L$

Level: (TRACE/LOW/MED) TRACE

o Moisture: not dec.

GC Colutun: DB-624

ID: $0.20 \quad(\mathrm{~mm})$

Soil Extract Volume:

(mL)

Purge Volume: 25.0
Lab Sample ID: 200-7357-5

Lab File ID: DHTA04.D

Date Recejved: $\quad 10 / 05 / 2011$

Date Analyzed: 10/07/2011

Dilution Factor: 1.0

Soil Aliquot Volume:

(uL)

\begin{tabular}{|c|c|c|c|}
\hline CAS NO. & COMPOUND & $\begin{array}{l}\text { CONCENTRATION UNITS: } \\
(u \mathrm{~g} / \mathrm{L} \text { or } \mathrm{ug} / \mathrm{kg}) \mathrm{ug} / \mathrm{L}\end{array}$ & $Q$ \\
\hline $79-01-6$ & Trichloroethene & 0.50 & $\mathrm{U}$ \\
\hline $108-87-2$ & Methylcyclonexane & 0.50 & U \\
\hline $78 \cdot-87-5$ & 1,2-Dichioropropane & 0.50 & $\mathrm{U}$ \\
\hline $75-27-4$ & Bromodichloromethane & 0.50 & U \\
\hline $10061-01-5$ & cis-1,3-Dichloropropene & 0.50 & $\mathrm{U}$ \\
\hline $108-10-1$ & 4-Methyl-2-pentanone & 5.0 & $\mathrm{U}$ \\
\hline $108-88-3$ & Toluene & 0.15 & $\mathrm{~J} \mathrm{~B}$ \\
\hline $10061-02-6$ & trans-1,3-Dichloropropene & 0.50 & (1) \\
\hline $79-00-5$ & 1,1,2-Trichloroethane & 0.50 & U \\
\hline $127-18-4$ & Tetrachloroethene & 0.50 & $\mathrm{U}$ \\
\hline $591-78-6$ & 2-Hexanone & 5.0 & $\mathrm{U}$ \\
\hline $124-48-1$ & Dibromochloromethane & 0.50 & $\mathrm{U}$ \\
\hline $106-93-4$ & 1,2-Dibromoethane & 0.50 & $\mathrm{U}$ \\
\hline $108-90-7$ & Chlorobenzene & 0.50 & $\mathrm{U}$ \\
\hline $100-41-4$ & Ethylbenzene & 0.016 & $\mathrm{~J}$ \\
\hline $95-47-6$ & o-xylenc & 0.032 & $J$ \\
\hline $179601-23-1$ & $m, p-x y \perp e n e$ & 0.042 & $\mathrm{~J} B$ \\
\hline $100-42-5$ & styrene & 0.030 & J \\
\hline $75-25-2$ & Bromoforil & 0.50 & $\mathrm{U}$ \\
\hline $98-82-8$ & Isopropylbenzere & 0.50 & $\mathrm{U}$ \\
\hline $79-34-5$ & $1,1,2,2$-Tetrachloroethane & 0.50 & $\mathrm{U}$ \\
\hline $541-73-1$ & 1,3-Dichlorobenzene & 0.024 & $J B$ \\
\hline $106-46-7$ & 1,4-Dichlorobenzene & 0.50 & U \\
\hline $95-50-1$ & 1,2-Lichlorobenzene & 0.50 & U \\
\hline $96-12-8$ & 1,2-Djbramo-3-Chloropropane & 0.50 & $\mathrm{U}$ \\
\hline $120-82-1$ & $1,2,4-T z 1$ chlorobenzene & 0.50 & U \\
\hline $87-61-6$ & $1,2,3-T$ richlorobenzene & 0.50 & U] \\
\hline
\end{tabular}


$1 \mathrm{~J}$ - FORM I VOA-TIC

VOLATILE ORGANICS ANALYSIS DATA SHEET TENTATIVELY IDENTIFIED COMPOUNDS
EPA SAMPLE NO.

MROCTB-W-30090

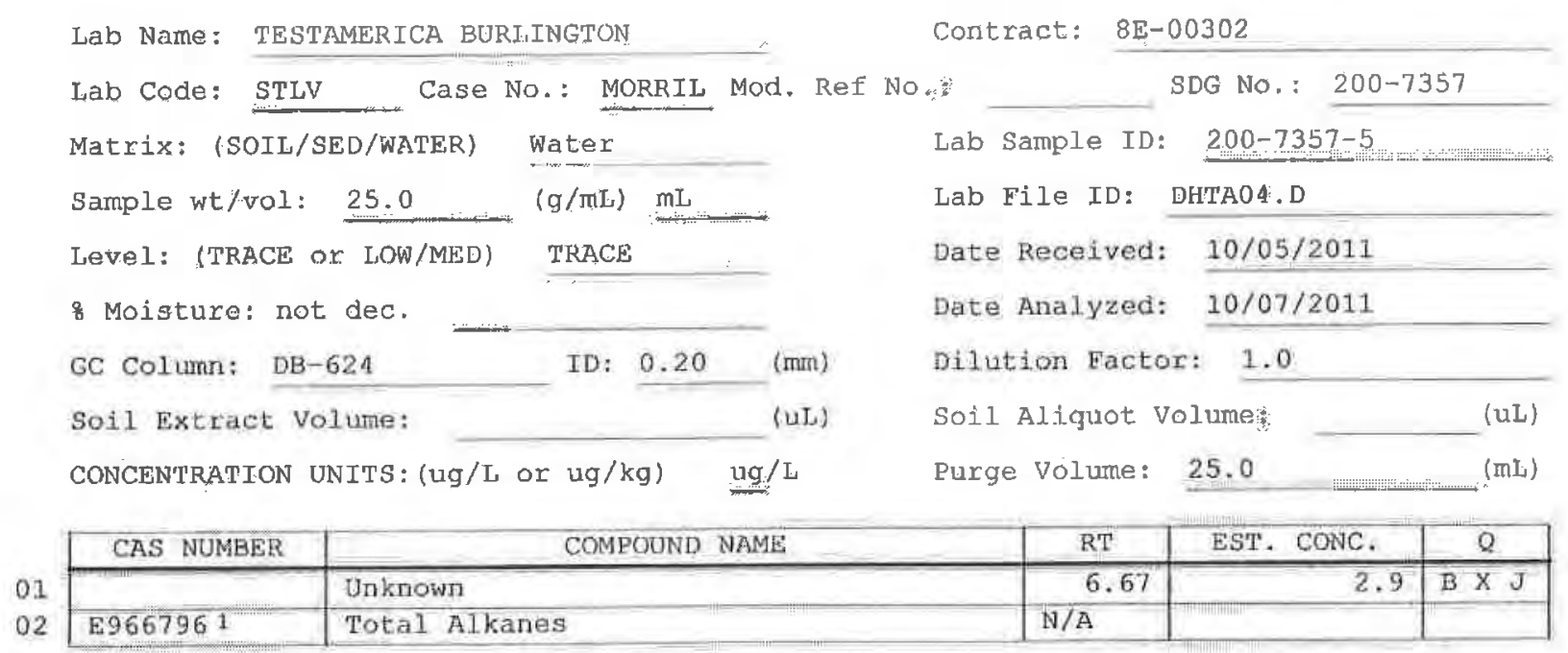

1 EPA-designated Registry Number. 
$1 A$ - FORM I VOA-1

VOJATILE ORGANTCS ANRIYSIS DATA SHEET
EPA SAMPLE NO.

MRSM2-W-3006.3

Iab Name: TESTAMERICA BURI,ING'YON

Contract: $\quad 8 \mathrm{E}-00302$

Lab Code: STLV Case No.: MORRIL Mod. Ref No.:

SDG No.: 200-7357

Matrix: (SOIL/SED/WATER) Water

Sample wt/vol: 25.0

( $\mathrm{g} / \mathrm{mL}) \mathrm{mL}$

Leve 1: (TRACE/LOW/MED) TRACE

\% Moisture: not dec.

GC Column: $\mathrm{DB}-624$

ID: $0.20 \quad(\mathrm{~mm})$

Soil. Extract Volume:

(uL)

Lab Sample ID; 200-7357-1

Lab File ID: DHTA05.D

Date Received: 10/05/2011

Date Aralyzed: 10/07/2011

Dilutjon Factor: 1.0

Soil Aliquot volume:

Purge Volume: 25.0

(mL)

\begin{tabular}{|c|c|c|c|}
\hline CAS NO. & COMPOUND & $\begin{array}{l}\text { CONCENIRATION UNITS: } \\
(\mathrm{ug} / \mathrm{L} \text { or } \mathrm{ug} / \mathrm{kg} \text { ) ug/L }\end{array}$ & $Q$ \\
\hline $75-71-8$ & Dichlorodifluoromethane & 0.50 & U \\
\hline $74-87-3$ & Chlorornethane & 0.50 & 0 \\
\hline $75-01-4$ & Vinyl chloride & 0.50 & $\mathrm{U}$ \\
\hline $74-83-9$ & Bromomethane & 0.50 & U \\
\hline $75-00-3$ & Chloroethane & 0.50 & $\mathrm{U}$ \\
\hline $75-69-4$ & Trichlorofluoromethane & 0.50 & U \\
\hline $75-35-4$ & 1,1-Dichloroethene & 0.50 & $\mathrm{U}$ \\
\hline $76-13-1$ & 1, 1,2-Trichloro-1,2,2-trifluoroethane & 0.50 & U \\
\hline $67-64-1$ & Acetone & 1.4 & J B \\
\hline $75-15-0$ & Carbon disulfide & 0.042 & $\mathrm{~J} B$ \\
\hline $79-20-9$ & Methyl acetate & 0.50 & $\mathrm{U}$ \\
\hline $75-09-2$ & Methylene Chloride & 0.50 & $\mathrm{U}$ \\
\hline $156-60-5$ & trans-1,2-Dichloroethene & 0.50 & $\mathrm{U}$ \\
\hline $1634-04-4$ & Methyl tert-butyl ether & 0.50 & U \\
\hline $75-34-3$ & 1,1-Dichloroethane & 0.50 & $\mathrm{U}$ \\
\hline $156-59-2$ & cis-1,2-Dichloroethene & 0.50 & U \\
\hline $78-93-3$ & 2-Butanone & 5.0 & $\mathrm{U}$ \\
\hline $74-97-5$ & Bromochloromethane & 0.50 & $\mathrm{U}$ \\
\hline $67-66-3$ & Chloroform & 0.50 & $\mathrm{U}$ \\
\hline $71-55-6$ & $1,1,1$-Irichloroethare & 0.50 & $\mathrm{U}$ \\
\hline $110-82-7$ & Cyclohexane & 0.50 & U \\
\hline $56-23-5$ & Carbon tetrachloride & 0.045 & J B \\
\hline $71-43-2$ & Benzene & 0.50 & $\mathrm{U}$ \\
\hline $107-06-2$ & 1,2-Dichloroethane & 0.50 & $\mathrm{U}$ \\
\hline
\end{tabular}

Report 1, 4-Dioxane for Low-Medium VoA analysis only 
$1 B$ - FORM I VOA-2

VOLATILE ORGBNICS ANALYYSIS DATA SHEET
EPA SAMPLE NO. MRSM2--W- 30063
Lab Name: 'I'ES'TAME'RICA BURLINGTON

Lab Code: STLV Case No.: MORRIJ Mod, Ref No: Matrix: (SOII/SED/WATER) Sample wt/vol: 25.0 Water Level: (TRACE/L.OW/MED) TRACE

Moisture: not dec.

GC Column: DB-624

Soil Extract Volume:

Purge Volume: 25.0
Contract: $8 \mathrm{E}-00302$ SDG No.: 200-7357

Lab Sample ID: 200-7357-1

IJab File ID: DH'lA05.D

Date Received: 10/05/2011

Date Analyzed: 10/07/2011

Dilution Factor: 1.0

Soil Aliquot Volume: (uL)

\begin{tabular}{|c|c|c|c|}
\hline CAS NO. & COMPOUND & $\begin{array}{l}\text { CONCENTRATION UNI'TS: } \\
(\mathrm{ug} / \mathrm{L} \circ \mathrm{or} \mathrm{ug} / \mathrm{kg}) \mathrm{ug} / \mathrm{L}\end{array}$ & $Q$ \\
\hline $79-01-6$ & Trichloroethene & 0.50 & $\mathrm{U}$ \\
\hline $108-87-2$ & Methylcyclohexane & 0.50 & U \\
\hline $78-87-5$ & 1,2-Dichloropropane & 0.50 & $\mathrm{U}$ \\
\hline $75-27-4$ & Bromodichloromethane & 0.50 & $\mathrm{U}$ \\
\hline $10061-01-5$ & cis-1,3-Dichloropropene & 0.50 & U \\
\hline $108-10-1$ & 4-Methyl-2-pentanone & 5.0 & U \\
\hline $108-88-3$ & Toluene & 0.24 & $\mathrm{~J} B$ \\
\hline $10061-02-6$ & trans-1, 3-Dichloropropene & 0.50 & $\mathrm{U}$ \\
\hline $79-00-5$ & 1,1,2-Trichloroethane & 0.50 & $\mathrm{U}$ \\
\hline $127-18-4$ & Tetrachloroethene & 0.50 & $\mathrm{U}$ \\
\hline $591-78-6$ & 2-Hexanone & 5.0 & $\mathrm{U}$ \\
\hline $124-48-1$ & Dibromochloromethane & 0.50 & $\mathrm{U}$ \\
\hline $106-93-4$ & 1,2-Dibromoethane & 0.50 & $\mathrm{U}$ \\
\hline $108-90-7$ & Chlorobenzene & 0.50 & 0 \\
\hline $100-41-4$ & Ethylbenzene & 0.50 & $\mathrm{U}$ \\
\hline $95-47-6$ & $0-x_{y}$ lene & 0.50 & $\mathrm{U}$ \\
\hline $179601-23-1$ & $m, p-X y l e n e$ & 0.0032 & $\mathrm{~J} \mathrm{~B}$ \\
\hline $100-42-5$ & Styrene & 0.50 & $\mathrm{U}$ \\
\hline $75-25-2$ & Bromoform & 0.50 & $\mathrm{U}$ \\
\hline $98-82-8$ & Isopropylbenzene & 0.50 & $\mathrm{U}$ \\
\hline $79-34-5$ & $1,1,2,2$-Tetrachloroethane & 0.50 & $\mathrm{U}$ \\
\hline $541-73-1$ & 1,3-Dichlorobenzene & 0.50 & $\mathrm{U}$ \\
\hline $106-46-7$ & 1,4-Dichlorobenzene & 0.50 & $\mathrm{U}$ \\
\hline $95-50-1$ & 1,2-Dichlorobenzene & 0.50 & $\mathrm{U}$ \\
\hline $96-12-8$ & 1,2-Dibromo-3-ChLoropropane & 0.50 & $\mathrm{U}$ \\
\hline $120-82-1$ & 1,2,4-Trichlorobenzene & 0.50 & $\mathrm{U}$ \\
\hline $87-61-6$ & 1,2,3-Trichlorobenzene & 0.50 & $\mathrm{U}$ \\
\hline
\end{tabular}


$1 \mathrm{~J}$ - FORM I VOA-TIC

VOLATILE ORGANICS ANALYSIS DATA SHEET

TENTA'TVELY IDENTIFIED COMPOUNDS
EPA SAMPLE NO.

MRSM2-W-30063

Lab Name: TESTAMERICA BURLINGTON

Contract: $8 \mathrm{E}-00302$

Lab Code: STLV Case No.: MORRIL Mod, Ref No.: SDG No.: $200-7357$

Matrix: (SOIL/SED/WATER) Water

Sample wt/vol: 25.0

$\left(g / m I_{1}\right) \quad r r L$

Leve1: (TRACE or LOW/MED)

TRACE

a Moisture: not dec:

GC Column: DB-624

ID: 0.20 (mu)

Soil Extract Volume: (uL)

CONCENTRATION UNITS: (ug/L or $\mathrm{ug} / \mathrm{kg}) \mathrm{ug} / \mathrm{L}$
Lab Sample ID: 200-735\%-1

Lab File ID: DHTA05.D

Date Received: 10/05/2011

Date Analyzed: 10/07/2011

Dilution Factor: 1.0

Soil Aliquot Volume:

(uL)

Purge volume: 25.0

02

\begin{tabular}{|l|l|r|r|r|}
\hline CAS NUMBER & \multicolumn{1}{|c|}{ COMPOUND NAME } & RT & EST. CONC. & Q \\
\hline & Unknown & 6.67 & 3.0 & $B \times 3$ \\
\hline E9667961 & Total Alkanes & N/A & & \\
\hline
\end{tabular}

${ }^{1}$ EPA-designated Registry Number. 
$6 \Lambda$ - TORM VT. VOA-1

VOLATILE ORGANICS INIIIAI, CALIBRATION DATA

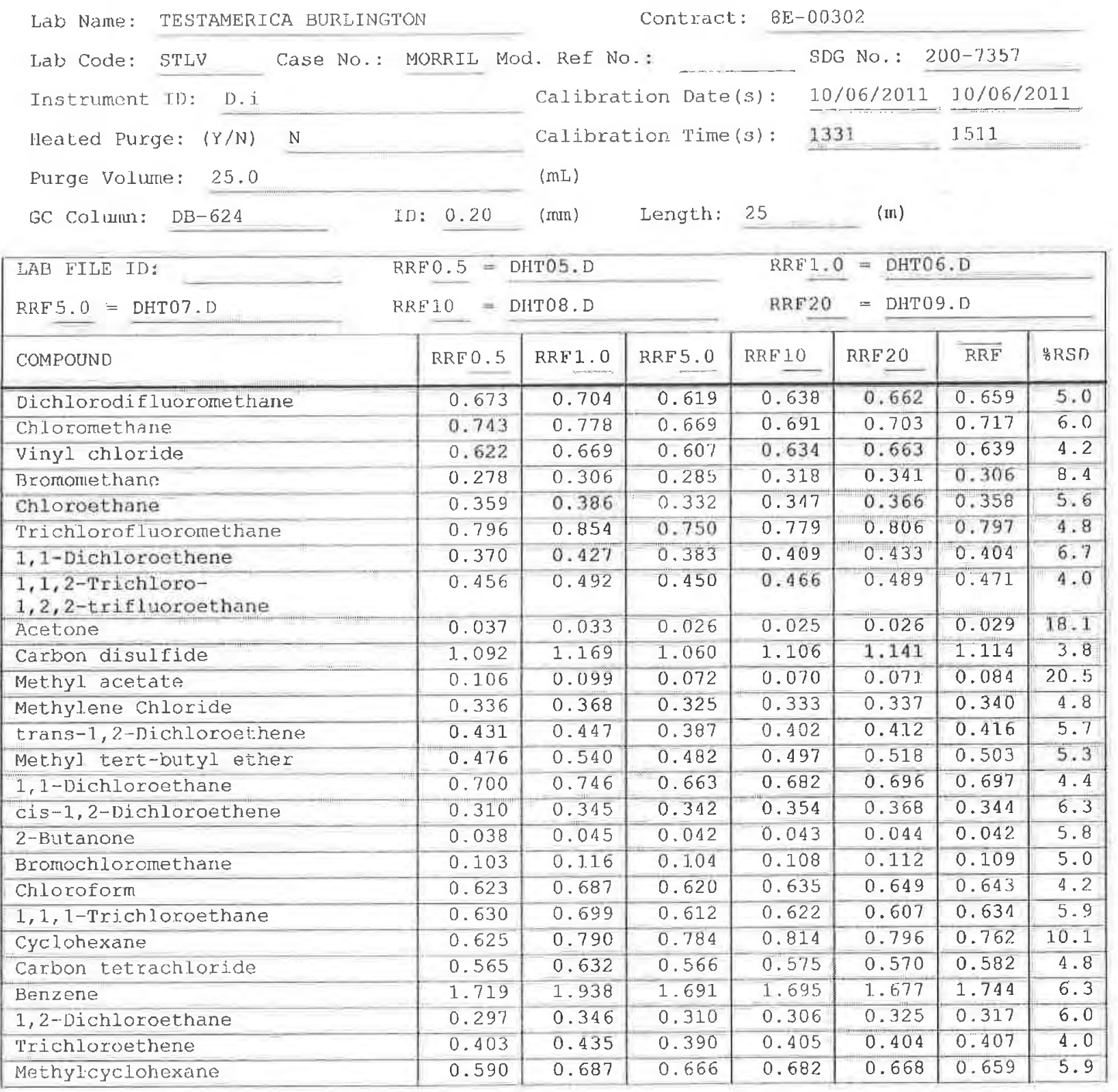

Report 1,4-Dioxane for Low-Medium VoA analysis only 
$6 B$ - FORM VI VOA-2

VOIATILE ORGANICS INITIAL CALIBRAPION DATA

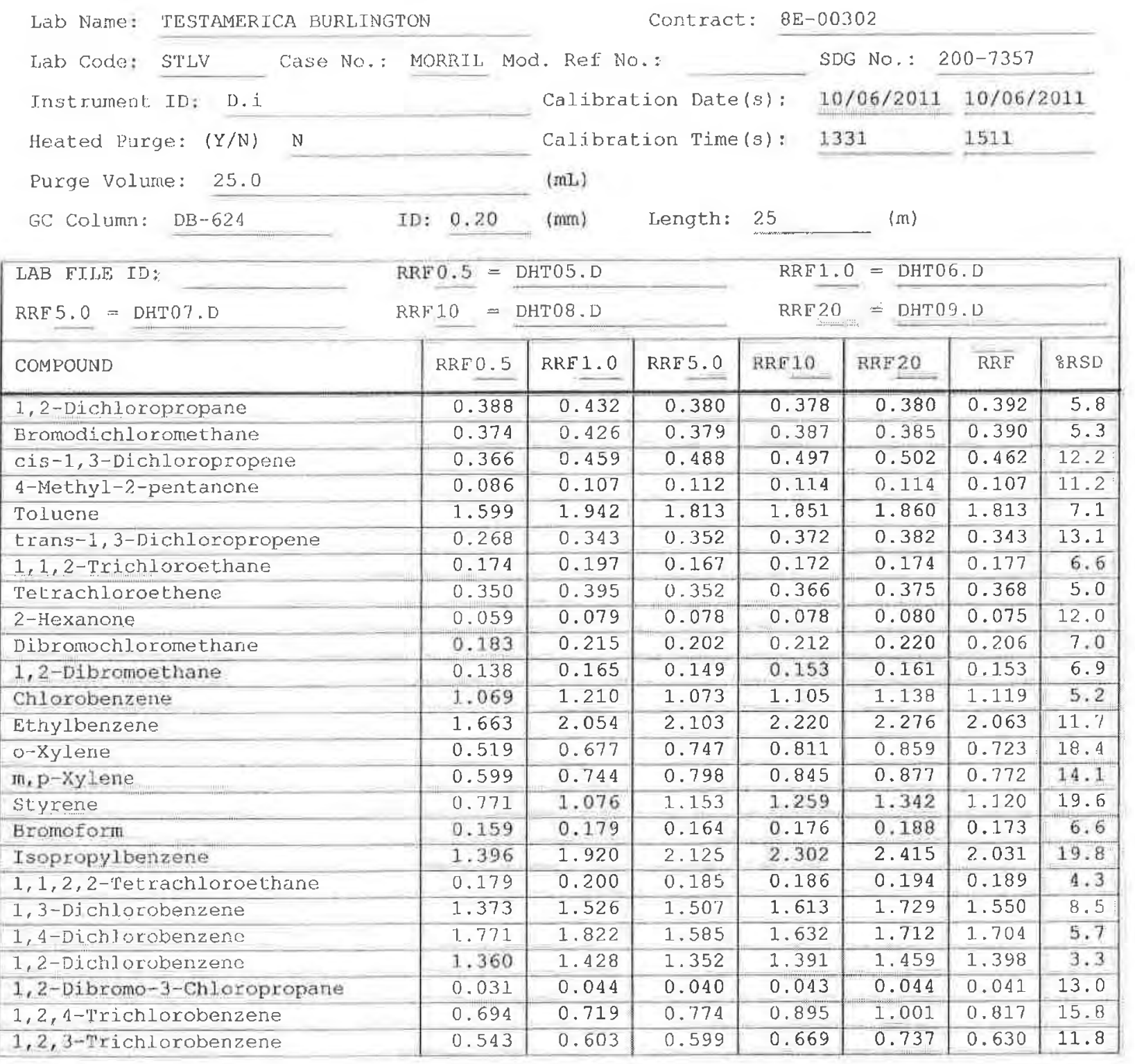


6C - FORM VI VOA-3

VOLATILE ORGANICS INITIAL CALIBRATION DATA

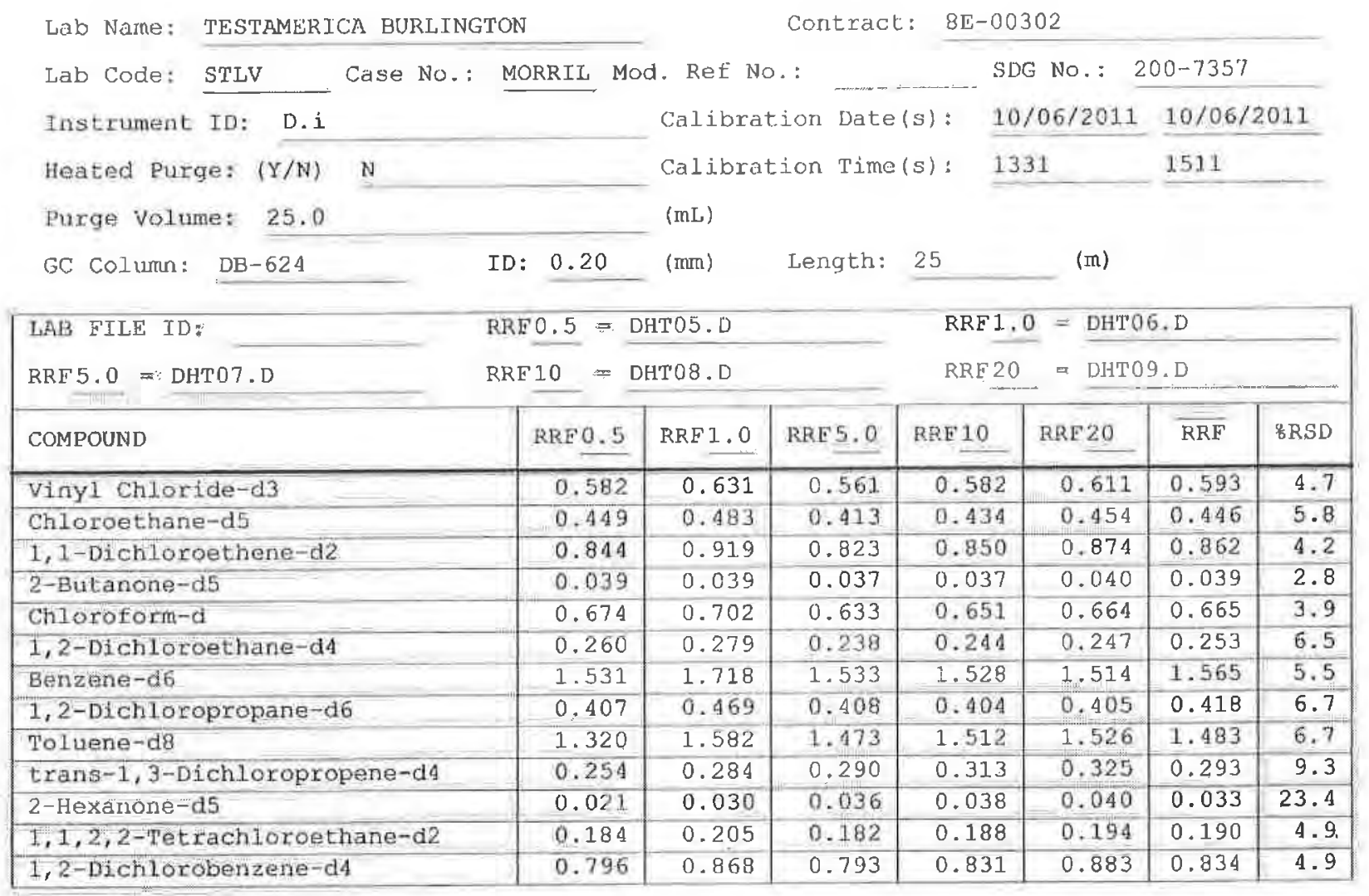

Report 1,4-Dioxane-d8 for Low-Medium VOA analysis only 
$7 A$ - EORM VII VOA-1

VOLATILF CONIINUING CALTBRATION DATA

Lab Name: TESTAMERICA BURLINGTON

Contract: $\quad 8 E-00302$

Lab Code: STLV Case No.: MORRIL Mod. Ref No.:

SDG No.: 200-7357

Instrument ID: D.i

Calibration Date: 10/07/2011 Tjme: 0730

Lab File Id: DHTA02.D Inít. Calib. Date(s): 10/06/2011 10/06/2011

EPA Sample No. (VSTD\#\#\#\#): VSTD005DJ

Init. Calib. Time(s): $1331 \quad 1511$

Heated Purge: ( $\mathrm{Y} / \mathrm{N})$

CC Column: DB-624

ID $: 0.20(\mathrm{~mm})$ Length: 25 (m)

Purge Volume: 25.0

(mL)

\begin{tabular}{|c|c|c|c|c|c|}
\hline COMPOUND & $\overline{\mathrm{RRE}}$ & RRE 5.0 & $\begin{array}{l}\text { MIN } \\
\text { RRE }\end{array}$ & $8 \mathrm{D}$ & MAX $\because D$ \\
\hline Dichlorodifluoromethane & 0.659 & 0.648 & 0.010 & -1.7 & 40.0 \\
\hline Chlorome thane & 0.717 & 0.705 & 0.010 & -1.7 & 40.0 \\
\hline Vinyl chloride & 0.639 & 0.626 & 0.010 & -2.0 & 30.0 \\
\hline Bromomethane & 0.306 & 0.308 & 0.010 & 0.7 & 30.0 \\
\hline Chloroethane & 0.358 & 0.347 & 0.010 & -3.2 & 40.0 \\
\hline Trichlorofluoromethane & 0.797 & 0.781 & 0.010 & -2.0 & 40.0 \\
\hline 1,1-Dichloroethene & 0.404 & 0.397 & 0.010 & -1.8 & 30.0 \\
\hline 1,1,2-Trichloro-1,2,2-trifluoroethane & 0.471 & 0.470 & 0.010 & -0.2 & 40.0 \\
\hline Acetone & 0.029 & 0.022 & 0.010 & -23.7 & 40.0 \\
\hline Carbon disulfide & 1.114 & 1.127 & 0.010 & 1.2 & 40.0 \\
\hline Methyl acetate & 0.084 & 0.067 & 0.010 & -19.5 & 40.0 \\
\hline Methylene Chloride & 0.310 & 0.335 & 0.010 & -1.4 & 40.0 \\
\hline trans-1,2-Dichloroethene & 0.416 & 0.365 & 0.010 & -12.3 & 40.0 \\
\hline Methyl tert-butyl ether & 0.503 & 0.396 & 0.010 & $-21 \cdot 3$ & 40.0 \\
\hline 1,1-Dichloroethane & 0.697 & 0.671 & 0.010 & -3.8 & 30.0 \\
\hline cis-1,2-Dichloroethene & 0.344 & 0.338 & 0.010 & -1.7 & 40.0 \\
\hline 2-Butanone & 0.042 & 0.035 & 0.010 & -16.5 & 40.0 \\
\hline Bromochloromethane & 0.109 & 0.103 & 0.010 & -4.8 & 30.0 \\
\hline Chloroform & 0.643 & 0.617 & 0.010 & -4.1 & 30.0 \\
\hline 1,1,1-Trichloroethane & 0.634 & 0.636 & 0.010 & $0.3^{\circ}$ & 30.0 \\
\hline Cyclohexane & 0.762 & 0.817 & 0.010 & 7.2 & 40.0 \\
\hline Carbon tetrachloride & 0.582 & 0.596 & 0.010 & 2.4 & 30.0 \\
\hline Benzene & 1.744 & 1.765 & 0.010 & 1.2 & 30.0 \\
\hline 1,2-Dichloroethane & 0.317 & 0.294 & 0.010 & -7.3 & 30.0 \\
\hline Trichloroethene & 0.407 & 0.406 & 0.010 & -0.3 & 30.0 \\
\hline Methylcyclohexane & 0.659 & 0.699 & 0.010 & 6.2 & 40.0 \\
\hline
\end{tabular}

Report 1,4-Dioxane for Jow/Medium VOA analysis only 
$7 B$ - EORM VII VOA-2

VOJ_ATILE CONTINUING CAIITBRATION DATA

Lab Name: TFSTAMERICA BURLINGTON

Contract: $8 \mathrm{E}-00302$

Lab Code: STLV Case No.: MORRIL Mod. Ref No.:

SDG No.: 200-735\%

Instrument ID: D. I

Calibration Date: 10/07/201.1 Time: 0730

Lab Eile Id: DHTAO2.D Init. Calib. Date(s): 10/06/2011 10/06/2011

EPA Sample No. (VSTD\#\#\#\#): VSTD005DJ

Init. Calib. Time(s): $1331 \quad 1511$

Heated Purge: (Y/N) N $\quad$ GC Column: DB-624 ID: 0.20 (mu) Length: 25 (m)

Puxge Volume: 25.0

(mL)

\begin{tabular}{|c|c|c|c|c|c|}
\hline COMPOUND & $\overline{\mathrm{RRE}}$ & RRE5.0 & $\begin{array}{l}\text { MIN } \\
\text { RRE }\end{array}$ & $8 \mathrm{D}$ & MAX $\& D$ \\
\hline 1,2-Dichloropropare & 0.392 & 0.383 & 0.010 & $-2,2$ & 40.0 \\
\hline Bromodichloromethane & 0.390 & 0.375 & 0.010 & -4.0 & 30.0 \\
\hline cis-1,3-Dichloropropene & 0.462 & 0.479 & 0.010 & 3.7 & 30.0 \\
\hline 4-Methyl-2-pentanone & 0.107 & 0.099 & 0.010 & -7.2 & 10.0 \\
\hline Toluene & 1.813 & 1.875 & 0.010 & 3.4 & 30.0 \\
\hline trans-1, 3-Dichloropropene & 0.343 & 0.340 & 0.010 & -0.9 & 30.0 \\
\hline $1,1,2$-Trichloroethane & 0.177 & 0.161 & 0.010 & -9.0 & 30.0 \\
\hline Tetrachloroethene & 0.368 & 0.367 & 0.010 & -0.3 & 30.0 \\
\hline 2-Hexanone & 0.075 & 0.069 & 0.010 & -8.5 & 40.0 \\
\hline Dibromochloromethane & 0.206 & 0.190 & 0.010 & -8.1 & 30.0 \\
\hline 1,2-Dibromoethane & 0.153 & 0.140 & 0.010 & -8.3 & 40.0 \\
\hline Chlorobenzene & 1.119 & 1.089 & 0.010 & -2.7 & 30.0 \\
\hline Ethylbenzene & 2.063 & 2.175 & 0.010 & 5.4 & 30.0 \\
\hline o-xylene & 0.723 & 0.763 & 0.010 & 5.6 & 30.0 \\
\hline $\mathrm{m}, \mathrm{p}$-xylene & 0.772 & 0.820 & 0.010 & 6.1 & 30.0 \\
\hline Styrene & 1.120 & 1.169 & $0.0 \pm 0$ & $4 \cdot 4$ & 30.0 \\
\hline Bromoform & 0.173 & 0.159 & 0.010 & $-8,1$ & 30.0 \\
\hline Isopropylbenzene & 2.031 & 2.208 & 0.010 & 8.7 & 40.0 \\
\hline $1,1,2,2$-Tetrachloroethane & 0.189 & 0.169 & 0.010 & $-10 \cdot 6$ & 30.0 \\
\hline 1,3-Dichlorobenzene & 1.550 & 1.501 & 0.010 & -3.1 & 30.0 \\
\hline 1,4-Dichlorobenzene & $1: 704$ & 1.603 & 0.010 & -5.9 & 30.0 \\
\hline 1,2-Dichlorobenzene & 1.398 & 1.330 & 0.010 & -4.9 & 30.0 \\
\hline 1,2-Dibromo-3-Chloropropane & 0.041 & 0.035 & 0.010 & -13.1 & 40.0 \\
\hline 1,2,4-Trichlorobenzene & 0.817 & 0.732 & 0.010 & $-10,3$ & 30.0 \\
\hline $1,2,3$-1'richlorobenzene & 0.630 & 0.566 & 0.010 & -10.2 & 30.0 \\
\hline
\end{tabular}


$7 C$ - FORM VII VOA-3

VOLATILE CONTINUING CALIBRAIIION DATA

Lab Name: 'TESTAMERICA BURLING'ION

Contract: $8 \mathrm{E}-00302$

Lab Code: STLV Case No.: MORRIL Mod. Ref No.:

SDG No.: 200-7357

Instrument. ID: D.1

Lab File Id: DHTAO2.D

Calibration Date: 10/07/2011 Tine: 0730

EPA Sample No. (VSTD\#\#\#\#)

Init. Calib. Date(s): 10/06/2011 10/06/2011

Heated purge: $(\mathrm{Y} / \mathrm{N}) \mathrm{N}$

VSTD005DJ

Init. Calib. Time(s): 1331

1511

Purge Volume: 25.0 GC Column: DB-624

ID: $0.20(\mathrm{~mm})$ Length: 25

(m)

\begin{tabular}{|c|c|c|c|c|c|}
\hline COMPOUND & $\overline{R R F}$ & RRE5.0 & $\begin{array}{l}\text { MIN } \\
\text { RRF }\end{array}$ & $8 \partial \mathrm{D}$ & MAX \&D \\
\hline Vinyl Chloride-d3 & 0.593 & 0.578 & 0.010 & -2.6 & 30.0 \\
\hline Chloroethane-d5 & 0.446 & 0.429 & 0.010 & -3.8 & 40.0 \\
\hline 1,1-Dichloroethene-d2 & 0.862 & 0.854 & 0.010 & -0.9 & 30.0 \\
\hline 2-Butanone-d5 & 0.039 & 0.031 & 0.010 & -19.0 & 40.0 \\
\hline Chloroform-d & 0.665 & 0.642 & 0.010 & -3.5 & 30.0 \\
\hline 1,2-Dichloroethane-dd & 0.253 & 0.229 & 0.010 & -9.6 & 30.0 \\
\hline Benzene-d6 & 1.565 & 1.575 & 0.010 & 0.6 & 30.0 \\
\hline 1,2-Dichloropropane-d6 & 0.418 & 0.406 & 0.010 & -3.0 & 40.0 \\
\hline Toluene-d8 & 1.483 & 1.536 & 0.010 & 3.6 & 30.0 \\
\hline trans-1,3-Dichloropropene-d4 & 0.293 & 0.277 & 0.010 & -5.5 & 30.0 \\
\hline $2-$ Hexanone-d5 & 0.033 & 0.031 & 0.010 & -7.3 & 40.0 \\
\hline $1,1,2,2$-Tetrachloroethane-d2 & 0.190 & 0.168 & 0.010 & -12.0 & 30.0 \\
\hline 1,2-Dichlorobenzene-d4 & 0.834 & 0.774 & 0.010 & -7.2 & 30.0 \\
\hline
\end{tabular}

Report 1,4-Dioxane-d8 for Low/Medium VOA analysis only 
$7 A$ - FORM VII VOA-1

VOJATILE CONTINUING CALIBRATION DA'IA

Lab Name: 'TESTRMERICA BURLINGTON

Contract: $8 \mathrm{E}-00302$

Lab Code: STLV Case No.; MORRIL Mod, Ref No.

SDG NO.: 200-7357

Instrument ID: D.i

Calibration Date: 10/07/201L Time: 1844

Lab File Jd: DHTA25.D

Init. Calib. Date(s): 10/06/2011 10/06/2011.

EPA Sample No. (VSTD\#\#\#\#): VSTD005JD

Init. Calib. Time(s): 1331

1511

Heated Furge: $(\mathrm{Y} / \mathrm{N}) \mathrm{N}$

GC Colums: DB-624

ID: $0.20(\mathrm{~mm})$ Length: 25

(m)

Purge Volume: 25.0 (mL)

\begin{tabular}{|c|c|c|c|c|c|}
\hline COMPOUND & $\overline{\mathrm{RRE}}$ & RRF5.0 & $\begin{array}{l}\text { MIN } \\
\text { RRF }\end{array}$ & $8 \mathrm{D}$ & $M A X \quad z D$ \\
\hline Dichlorodifluoromethane & 0.659 & 0.602 & 0.010 & -8.7 & 50.0 \\
\hline Chloromethane & 0.717 & 0.660 & 0.010 & -8.0 & 50.0 \\
\hline VinyI chloride & 0.639 & 0.584 & 0.100 & -8.6 & 50.0 \\
\hline Bromomethane & 0.306 & 0.286 & 0.100 & -6.5 & 50.0 \\
\hline Chloroethane & 0.358 & 0.324 & 0.010 & -9.6 & 50.0 \\
\hline Trichlorofluoromethane & 0.797 & 0.728 & 0.010 & -8.6 & 50.0 \\
\hline 1,1-Dichloroethene & 0.404 & 0.376 & 0.100 & -7.0 & 50.0 \\
\hline 1,1,2-Trichloro-1,2,2-trifluoroethane & 0.471 & 0.447 & 0.010 & -5.0 & 50.0 \\
\hline Acetone & 0.029 & 0.021 & 0.010 & -30.1 & 50.0 \\
\hline Carbon disulfide & 1.114 & 1.068 & 0.010 & -4.1 & 50.0 \\
\hline Methyl acetate & 0.084 & 0.063 & 0.010 & $-24 \cdot 9$ & 50.0 \\
\hline Methylene Chloride & 0.340 & 0.301 & 0.010 & -11.4 & 50.0 \\
\hline trans $-1,2-$ Dichloroethene & 0.416 & 0.329 & 0.010 & -20.9 & 50.0 \\
\hline Methyl tert-butyl ether & 0.503 & 0.337 & 0.010 & -32.9 & 50.0 \\
\hline 1,1-Dichloroethane & 0.697 & 0.623 & 0.200 & -10.7 & 50.0 \\
\hline cis-1,2-Dichloroethene & 0.344 & 0.309 & 0.010 & -10.0 & 50.0 \\
\hline 2-Butanone & 0.042 & 0.030 & 0.010 & -28.3 & 50.0 \\
\hline Bromochloromethane & 0.109 & 0.090 & 0.050 & -17.1 & 50.0 \\
\hline Chloroform & 0.643 & 0.568 & 0.200 & -11.7 & 50.0 \\
\hline 1,1,1-Trichloroethane & 0.634 & 0.580 & 0.100 & -8.5 & 50.0 \\
\hline Cyciohexane & 0.762 & 0.749 & 0.010 & -1.7 & 50.0 \\
\hline Carbon tetxachloride & 0.582 & 0.555 & 0.100 & -4.6 & 50.0 \\
\hline Benzene & 1.744 & 1.602 & 0.400 & -8.1 & 50.0 \\
\hline 1,2-Dichloroethane & 0.317 & 0.267 & 0.100 & -.15 .7 & 50.0 \\
\hline Trichloroethene & 0.407 & 0.373 & 0.300 & -8.5 & 50.0 \\
\hline Methylcyclohexane & 0.659 & 0.643 & 0.010 & -2.3 & 50.0 \\
\hline
\end{tabular}

Report 1,4-Dioxane for Low/Medium VOA analysis only 
$7 B$ - BORM VII VOA-2

VOLAIILE CONTINUING CALIBRATION DATA

Lab Name: TESTAMERICA BURLINGTON

Contract: $8 \mathrm{E}-00302$

Lab Code: STLy Case No.: MORRIL Mod, Ref No.

SDG No.: 200-7357

Instrument ID: D.i

Calibration Date: 10/07/2011. Time: 1844

Lab File Id: DHTA25.D Init. Calib. Date(s): 10/06/2011 10/06/2011

EPA Sample No. (VSTD\#\#\#\#): VSTD00SJD

Init. Calib. Time(s): $1331 \quad 1511$

Heated Purge: $(\mathrm{Y} / \mathrm{N})$

GC Column: DB-624

ID: 0.20 (Im) Length: 25 (m)

Purge Volume: 25.0

(mL)

\begin{tabular}{|c|c|c|c|c|c|}
\hline COMPOUND & $\overline{\mathrm{RRF}}$ & RRE5. 0 & $\begin{array}{l}\text { MIN } \\
\text { RRE }\end{array}$ & $\& D$ & $\operatorname{MAX} \quad \mathrm{O} D$ \\
\hline 1,2-Dichloropropane & 0.392 & 0.339 & 0.07 .0 & -13.4 & 50.0 \\
\hline Bronodichloromethane & 0.390 & 0.332 & 0.200 & -15.0 & 50.0 \\
\hline cis-1,3-Dichloropropene & 0.462 & 0.424 & 0.200 & -8.4 & 50.0 \\
\hline 4-Methyl-2-pentanone & 0.107 & 0.083 & 0.010 & -22.4 & 50.0 \\
\hline Toluene & 1.813 & 1.719 & 0.400 & -5.2 & 50.0 \\
\hline trans $-1,3$-Dichloropropene & 0.343 & 0.295 & 0.100 & -14.2 & 50.0 \\
\hline 1, 1,2-Trichloroethane & 0.177 & 0.144 & 0.100 & -18.5 & 50.0 \\
\hline Tetrachloroethene & 0.368 & 0.332 & 0.100 & -9.7 & 50.0 \\
\hline 2-Hexanone & 0.075 & 0.056 & 0.010 & -24.9 & 50.0 \\
\hline Dibromochloromethane & 0.206 & 0.170 & 0.100 & -17.8 & 50.0 \\
\hline 1.2-Dibromoethane & 0.153 & 0.121 & 0.010 & -20.7 & 50.0 \\
\hline Chlorobenzene & 1.119 & 0.978 & 0.500 & -12.6 & 50.0 \\
\hline Ethylbenzene & 2.063 & 1.994 & 0.100 & -3.3 & 50.0 \\
\hline o-Xylene & 0.723 & 0.687 & 0.300 & -5.0 & 50.0 \\
\hline $\mathrm{m}, \mathrm{p}$-Xylene & 0.772 & 0.745 & 0.300 & -3.5 & 50.0 \\
\hline Styrene & 1.120 & 1.058 & 0.300 & $-5 . \overline{6}$ & 50.0 \\
\hline Bromoform & 0.173 & 0.133 & 0.050 & -23.0 & 50.0 \\
\hline Isopropy lbenzene & 2.031 & 2.010 & 0.010 & -1.1 & 50.0 \\
\hline $1,1,2,2$-Tetrachloroethane & 0.189 & 0.146 & 0.100 & -22.7 & 50.0 \\
\hline 1,3-Dichlorobenzene & 1.550 & 1.343 & 0.400 & -13.3 & 50.0 \\
\hline 1, 1-Dichlorobenzene & 1.701 & 1.471 & 0.400 & -13.7 & 50.0 \\
\hline 1,2-Dichlorobenzene & 1. 398 & 1.175 & 0.400 & -15.9 & 50.0 \\
\hline 1,2-Dibromo-3-ChJ oropropane & 0.041 & 0.030 & 0.010 & -25.3 & 50.0 \\
\hline 1,2,4-Trichlorobenzene & 0.817 & 0.635 & 0.200 & -22.3 & 50.0 \\
\hline 1,2,3-Trichloxobenzene & 0.630 & 0.487 & 0.200 & -22.6 & 50.0 \\
\hline
\end{tabular}


7C - EORM VII VOA-3

VOLATILE CONTINUING CALIBRAIION DATA

Lab Name: TESTAMERICA BURLTINGTON

Contract: $8 \mathrm{E}-00302$

Lab Code: STLV Case No.: MORRIL Mod. Ref No.:

SDG NO: : 200-7357

Instrument ID: D.i

Calibration Date: 10/07/2011 Time: 1844

Lab File Id: DHTA25,D Init. Calib. Date(s): 10/06/2011, 10/06/2011

EPA Sample No. (VSTD\#\#\#\#): VSTD005.JD

Init. Calib. Time(s): $1331 \quad 1511$

Heated Purge: (Y/N) N GC Column: DB-624 ID: 0.20 (mm) Length: 25 (m)

Purge Volume: 25.0 (mL)

\begin{tabular}{|c|c|c|c|c|c|}
\hline COMPOUND & $\overline{\mathrm{RRF}}$ & RRE5. 0 & $\begin{array}{l}\text { MIN } \\
\text { RRF }\end{array}$ & $8 \mathrm{D}$ & $\operatorname{MAX} \div \mathrm{D}$ \\
\hline Vinyl Chloride-d3 & 0.593 & 0.535 & 0.010 & -9.8 & 50.0 \\
\hline Chloroethane-d5 & 0.446 & 0.406 & 0.010 & -9.0 & 50.0 \\
\hline 1,1-Dichloroethene-d2 & 0.862 & 0.793 & 0.010 & -8.0 & 50.0 \\
\hline 2-Butanone-d5 & 0.039 & 0.026 & 0.010 & -33.4 & 50.0 \\
\hline Chloroform-d & 0.665 & 0.585 & 0.010 & -12.0 & 50.0 \\
\hline 1,2-Dichloroethane-d4 & 0.253 & 0.205 & 0.010 & -19.2 & 50.0 \\
\hline Benzene-d 6 & 1.565 & 1.442 & 0.010 & -7.8 & 50.0 \\
\hline 1,2-Dichloropropane-d6 & 0.418 & 0.364 & 0.010 & -13.0 & 50.0 \\
\hline Toluene-d8 & 1.483 & 1.399 & 0.010 & -5.7 & 50.0 \\
\hline trans-1,3-Dichloropropene-d4 & 0.293 & 0.243 & 0.010 & -17.0 & 50.0 \\
\hline 2-Hexanone-d5 & 0.033 & 0.024 & 0.010 & -26.4 & 50.0 \\
\hline $1,1,2,2$-Tetrachloroethane-d2 & 0.190 & 0.149 & 0.010 & -21.6 & 50.0 \\
\hline 1,2-Dichlorobenzene-d4 & 0.834 & 0.690 & 0.010 & -17.3 & 50.0 \\
\hline
\end{tabular}

Report 1,4-Dioxane-d6 For Low/Medium VoA analysis only 
$1 A$ - EORM I VOA-1

VOLATTIAF ORGANICS ANALYSIS DATA SHEET
EPA SAMPLE NO.

VBLKDJ
Lab Name: 'ПESTAMERICA BURLING'TON

Lab Code: STLLV_Case No.: MORRIL Mod. Ref No.:

Matrix: (SOIL/SED/WATER) Water

Sample wt/vol: $25.0 \ldots(\mathrm{g} / \mathrm{mL}) \mathrm{nL} \ldots$

Level: (TRACE/LOW/MED) TRACE,

옹 Moisture; not dec.

GC Column: DB-624 ID: 0.20 (mm)

Soil Extract Volune:

(uL)

$(\mathrm{mL})$

Purge volume: 25.0
Contract: $\quad 8 \mathrm{E}-00302$

SDG No.: 200-735?

Lab Sample ID: MB 200-26380/3

Lab File ID: DHTA03.D

Date Received:

Date Analyzed: $\quad 10 / 07 / 2011$

Dilution Factor: 1.0

Soil Aliquot Volume:

(uL)

COMPOUND

Dichlorodifluoromethane

Chloromethane

Vinyl chloride

Bromomethane

Chloroethane

Trichlorofluoromethane

$75-69-4$

1,1-Dichloroethene

$76-13-1$

$67-64-1$

1, 1,2-Irichloro-1, 2,2-trifluoroethane

Acetone

$75-15-0$

$79-20-9$

$75-09-2$

$156-60-5$

$1634-04-4$

$75-34-3$

$156-59-2$

$78-93-3$

$74-97-5$

$67-66-3$

$71-55-6$

$110-82-7$

$56-23-5$

$71-43-2$

$107-06-2$

Carbon disulfide

Methyl acetate

Methylene Chloride

trans-1,2-Dichloroethene

Methyl tert-butyl ether

1,1-Dichloroethane

cis-1,2-Dichloroethene

2-Butanone

Bromochloromethane

Chloroform

1, 1,1-Trichloroethane

Cyclohexane

Carbon tetrachloride

Benzene

1,2-Dichloroethane

\begin{tabular}{|c|c|}
\hline $\begin{array}{l}\text { CONCENTRATION UNI'TS: } \\
\text { (ug } / \mathrm{L} \text { or } \mathrm{ug} / \mathrm{kg} \text { ) } \mathrm{ug} / \mathrm{L}\end{array}$ & Q \\
\hline 0.50 & $\mathrm{U}$ \\
\hline 0.50 & 0 \\
\hline 0.50 & $\mathrm{U}$ \\
\hline 0.033 & $\mathrm{~J}$ \\
\hline 0.50 & U \\
\hline 0.50 & J \\
\hline 0.50 & U \\
\hline 0.50 & U \\
\hline 1.1 & J \\
\hline 0.086 & J \\
\hline 0.50 & U \\
\hline 0.039 & J \\
\hline 0.50 & U \\
\hline 0.50 & $\mathrm{U}$ \\
\hline 0.50 & $\mathrm{U}$ \\
\hline 0.50 & $\mathrm{U}$ \\
\hline 5.0 & U \\
\hline 0.50 & U \\
\hline 0.50 & $\mathrm{U}$ \\
\hline 0.50 & $\mathrm{U}$ \\
\hline 0.50 & $\mathrm{U}$ \\
\hline 0.018 & $\mathrm{~J}$ \\
\hline 0.50 & $\mathrm{U}$ \\
\hline 0.50 & U \\
\hline
\end{tabular}

Report 1,4-Dioxane for Low-Medium VOA analysis only 
13 - FORM I VOA-2

VOLATILE ORGANICS ANALYSIS DATA SHEET
EPA SAMPLE: NO. VBLKDJ

Lab Name: TESTAMERICA BURLINGTON

Contract: $\quad 8 E-00302$

Lab Code: STLV Case No.: MORRIL Mod. Ref No.:

SDG No.: 200-7357

Matrix: (SOIL/SED/WATER) Water

Sample wt/vol: $25.0 \quad(\mathrm{~g} / \mathrm{mJ}) \mathrm{mL}$

Level: (TRACE/LOW/MED) TRACE

Moisture: not dec.

GC Column: $\mathrm{DB}-624$

ID: 0.20 (mm)

Soi] Extract Volume: (UL)

Purge Volume: 25.0 (mL)
Lab Sample ID: MB 200-26380/3

Lab File ID: DHIA03.D

Date Received:

Date Analyzed: 10/07/2011

Dilution Eactor: 1.0

Soil Aliquot Volume: (uL)

\begin{tabular}{|c|c|c|c|}
\hline CAS NO: & COMPOUND & $\begin{array}{l}\text { CONCENTRATION UNITS: } \\
(\mathrm{ug} / \mathrm{L} \text { or } \mathrm{ug} / \mathrm{kg}) \mathrm{ug} / \mathrm{L}\end{array}$ & $Q$ \\
\hline $79-01-6$ & Trichloroethene & 0.50 & $\mathrm{U}$ \\
\hline $108-87-2$ & Methylcyclohexane & 0.50 & $\mathrm{t}$ \\
\hline $78-87-5$ & 1,2-Dichloropropane & 0.50 & $\mathrm{U}$ \\
\hline $75-27-4$ & Bromodichloromethane & 0.50 & $\mathrm{U}$ \\
\hline $10061-01-5$ & cis-1,3-Dichloropropene & 0.50 & $\mathrm{U}$ \\
\hline $108-10-1$ & 4-Methy1-2-pentanone & 5.0 & $\mathrm{U}$ \\
\hline $108-88-3$ & Toluene & 0.011 & $\mathrm{~J}$ \\
\hline $10061-02-6$ & trans-1,3-Dichloropropene & 0.50 & U \\
\hline $79-00-5$ & 1,1,2-Trichloroethane & 0.50 & $\mathrm{U}$ \\
\hline $127-1.8-4$ & Tetrachloroethene & 0.50 & $\mathrm{U}$ \\
\hline $591-78-6$ & 2-Hexanone & 5.0 & U \\
\hline $124-48-1$ & Dibromoch loromethane & 0.50 & $\mathrm{U}$ \\
\hline $106-93-4$ & 1,2-Dibromoethane & 0.50 & U \\
\hline $108-90-7$ & Chlorobenzene & 0.50 & 0 \\
\hline $100-41-4$ & Ethylbenzene & 0.50 & $\mathrm{U}$ \\
\hline $95-47-6$ & o-Xylene & 0.50 & $\mathrm{U}$ \\
\hline $179601-23-1$ & m, p-Xylene & 0.0038 & J \\
\hline $100-42-5$ & styrene & 0.50 & $\mathrm{U}$ \\
\hline $75-25-2$ & Bromoform & 0.50 & $\mathrm{U}$ \\
\hline $98-82-8$ & Isopropylbenzene & 0.50 & $\mathrm{U}$ \\
\hline $79-34-5$ & $1,1,2,2$-Tetrachloroethane & 0.50 & 0 \\
\hline $541-73-1$ & 1,3-Dichlorobenzene & 0.040 & $\mathrm{~J}$ \\
\hline $106-46-7$ & 1,4-Dichlorobenzene & 0.50 & $\mathrm{u}$ \\
\hline $95-50-1$ & 1,2-Dichlorobenzene & 0.50 & $\mathrm{U}$ \\
\hline $96-12-8$ & 1,2-Dibromo-3-Chloropropane & 0.50 & $\mathrm{U}$ \\
\hline $120-92-1$ & $1,2,4$-Trichlorobenzene & 0.074 & $\mathrm{~J}$ \\
\hline $87-61-6$ & $1,2,3-$ Trichlorobenzene & $0.07 \overline{9}$ & $\tilde{u}$ \\
\hline
\end{tabular}


$1 \mathrm{~J}$ - FORM I VOA-TIC

VOLATILE ORGANICS ANALYSIS DATA SHEET

TENTATJVELY IDENTIFIED COMPOUNDS
EPA SAMPLE NO.

VBIKDJ

Lab Name: TESTAMERICA BURLINGTON

Contract: $\quad 8 \mathrm{E},-00302$

lab code: STLV Case No.: MORRIL Mod. Ref No.: SDG No.: 200-7357

Matrix: (SOIL/SED/WATER) Water

Sample wt/vol: 25.0

Level: (TRACE or LOW/MED) TRACE

$(\mathrm{g} / \mathrm{mL}) \mathrm{mL}$

Foisture: not dec;

GC Column: DB-624

ID : 0.20 (mm)

Soil Extract Volume: (uL)

CONCENTRATION UNITS: (ug/L or $u g / k g) \quad u g / L$
Lab Sample ID: MB 200-26380/3

Lab File ID: DHTA03.D

Date Received:

Date Analyzed: 10/07/2011

Dilution Factor: 1.0

Soil Aliquot Volume:

Purge Volume: 25.0 (mL)

01

02

\begin{tabular}{|l|l|r|r|r|}
\hline \multicolumn{1}{|c|}{ CAS NUMBER } & \multicolumn{1}{|c|}{ COMPOUND NAME } & RT & EST. CONC. & Q \\
\hline & Unknown & 6.67 & $3.4 . \mathrm{J}$ \\
\hline E 9667961 & Total Alkanes & N/A & \\
\hline
\end{tabular}

LEPA-designated Registry Number. 
EPA SAMPLE NO.

VHBLLKO1

Lab Name: TESTAMERICA BURLINGTON

Contract: $8 \mathrm{E}-00302$

Lab code: STLV Case No.: MORRIL Mod. Ref No: :

SDG NO.: $\quad 200-7357$

Matrix: (SOIL/SED/WATER) Water

Sample wi/vol: 25.0

$(\mathrm{g} / \mathrm{mI}) \mathrm{mL}$

Level: (TRACE/LOW/MED) TRACE

goisture: not dec.

GC Column: DB-624

soil Extract volume:

ID: 0.20

(min)

(uL)

(mL)

Purge volume: 2.5 .0
Lab Sample ID: 200-7357-6

I.ab File ID: DHTA11.D

Date Received:

Date Analyzed: $10 / 07 / 2011$

Dilution Factor: 1.0

Soil Aliquot Volume:

(uL)

\begin{tabular}{|c|c|c|c|}
\hline CAS NO. & COMPOUND & $\begin{array}{l}\text { CONCENIRAIION [UNITS: } \\
(\mathrm{ug} / \mathrm{L} \text { or } \mathrm{ug} / \mathrm{kg}) \mathrm{ug} / \mathrm{L}\end{array}$ & $Q$ \\
\hline $75-71-8$ & Dichlorodifluoromethane & 0.50 & $\mathrm{U}$ \\
\hline $74-87-3$ & Chloromethane & 0.50 & $\mathrm{U}$ \\
\hline $75-01-4$ & Vinyl chloride & 0.50 & [1 \\
\hline $74-83-9$ & Bromomethane & 0.50 & U \\
\hline $75-00-3$ & Chloroethane & 0.50 & U \\
\hline $75-69-4$ & Trichlorofluoromethane & 0.50 & $\mathrm{U}$ \\
\hline $75-35-4$ & $1,1-$ DI.chloroethene & 0.50 & U \\
\hline $76-13-1$ & 1,1,2-Trichloro-1,2,2-trifluoroethane & 0.50 & $\mathrm{U}$ \\
\hline $67-64-1$ & Acetone & 5.0 & $\mathrm{U}$ \\
\hline $75-15-0$ & Carbon disulfide & 0.029 & $\mathrm{~J} \mathrm{~B}$ \\
\hline $79-20-9$ & Methyl acetate & 0.50 & U \\
\hline $75-09-2$ & Methylene Chloride & 0.50 & $\mathrm{U}$ \\
\hline $156-60-5$ & trans-1,2-Dichloroethene & 0.50 & U \\
\hline $1634-04-4$ & Methyl tert-butyl ether & 0.50 & $\mathrm{U}$ \\
\hline $75-34-3$ & 1,1-Dichloroethane & 0.50 & $\mathrm{U}$ \\
\hline $156-59-2$ & cis-1,2-Dichloroethene & 0.50 & $\mathrm{U}$ \\
\hline $78-93-3$ & 2-Butanone & 5.0 & U \\
\hline $74-97-5$ & Bromochloromethane & 0.50 & $\mathrm{U}$ \\
\hline $67-66-3$ & Chloroform & 0.50 & $\mathrm{U}$ \\
\hline $71-55-6$ & $1,1,1$-Trichloroethane & 0.50 & $\mathrm{U}$ \\
\hline $110-82-7$ & Cyclohexane & 0.50 & $U$ \\
\hline $56-23-5$ & Carbon tetrachloride & 0.017 & $\mathrm{~J} \mathrm{~B}$ \\
\hline $71-43-2$ & Benzene & 0.50 & U \\
\hline $107-06-2$ & 1,2-Dichloroethane & 0.50 & U \\
\hline
\end{tabular}

Report 1,4-Dioxane for Low-Medium VOA aralysis only 
$1 B$-. TORM I VOA-2

VOLATLE ORGANTCS ANALYSTS DALA SHEF'I
EPA SAMPIE NO.

VHBLKO1
Lab Name: IESTMMERJCA BURLINGTON

I.ab Code: STLV Case No.: MORRII Mod. Ref No.:

Matrix: (SOIL/SED/WATER) Water

Sample wt/vol: 25.0

$(g / m l) \quad m l$,

Leve].: (TRACE/I.OW/MED) TRACE

各 Moisture: not dec.

GC Column: DB-624

I D : 0.20

Soil Extract Volume:

Purge Volume: 25.0
Contract: $\quad$ 8E-00302

SDG No.: 200--7357

Lab Sample ID: $200-7357-6$

Tab File TD: DHTA11.D

Date Received:

Date Analyzed: 10/07/2011

Dilution Eactor: 1.0

Soil Aliquot Volume:

(uL)

\begin{tabular}{|c|c|c|c|}
\hline CAS NO. & COMPOUND & $\begin{array}{l}\text { CONCENTRATION UNITS: } \\
(\mathrm{ug} / \mathrm{I} \text { of } \mathrm{ug} / \mathrm{kg}) \mathrm{ug} / \mathrm{L}\end{array}$ & Q \\
\hline $79-01-6$ & Trichloroethene & 0.50 & U \\
\hline $108-87-2$ & Methylcyclohexane & 0.50 & $\mathrm{U}$ \\
\hline $78-87-5$ & 1,2-Dichloropropane & 0.50 & $\mathrm{U}$ \\
\hline $75-27-4$ & Bromodich loromethane & D. 50 & $\mathrm{U}$ \\
\hline $10061-01-5$ & cis-1,3-Dichloropropene & 0.50 & $\mathrm{U}$ \\
\hline $108-10-1$ & 4-Methyl-2-pentanone & 5.0 & U \\
\hline $108-88-3$ & Toluene & 0.50 & $\mathrm{U}$ \\
\hline $10061-02-6$ & trans-1, 3-Dichloropropene & 0.50 & U \\
\hline $79-00-5$ & 1,1,2-Trichloroethane & 0.50 & $\mathrm{U}$ \\
\hline $127-18-4$ & Tetrachloroethene & 0.50 & U \\
\hline $591-78-6$ & 2-Hexanorie & 5.0 & $\mathrm{U}$ \\
\hline $124-48-1$ & Dibromoch loronethane & 0.50 & $\mathrm{U}$ \\
\hline $106-93-4$ & 1,2-Dibromoethane & 0.50 & $\mathrm{U}$ \\
\hline $108-90-7$ & Chlorobenzene & 0.50 & $\mathrm{U}$ \\
\hline $100-41-4$ & Ethyibenzerie & 0.50 & $\mathrm{U}$ \\
\hline $95-47-6$ & o-XYiene & 0.50 & U \\
\hline $179601-23-1$ & $m, p$-Xylene & 0.50 & $\mathrm{U}$ \\
\hline $100-42-5$ & Styxene & 0.50 & U \\
\hline $75-25-2$ & Bromoform & 0.50 & U \\
\hline $98-82-8$ & Isopropylbenzene & 0.50 & $\mathrm{U}$ \\
\hline $79-34-5$ & $1,1,2,2$-Tetrachloroethane & 0.50 & $\mathrm{U}$ \\
\hline $541-73-1$ & 1,3-Dichlorobenzene & 0.50 & $\mathrm{U}$ \\
\hline $106-46-7$ & 1,4-Dichlorobenzene & 0.50 & U \\
\hline $95-50-1$ & 1,2-Dichlorobenzene. & 0.50 & $\mathrm{U}$ \\
\hline $96-12-8$ & 1,2-Dibromo-3-Chloropropane & 0.50 & $\mathrm{U}$ \\
\hline $120-82-1$ & 1,2,4-Tri.chlorobenzene & 0.50 & $\mathrm{U}$ \\
\hline $87-61-6$ & $1,2,3-$ Trichlorobenzene & 0.50 & $\mathrm{U}$ \\
\hline
\end{tabular}


$1 \mathrm{~J}$ - FORM I. VOA-TIC

VOLATILE ORGANICS ANAI

TENTATIVELY IDENTIFIED COMPOUNDS
EPA SAMPLE NO.

VHBLKOI

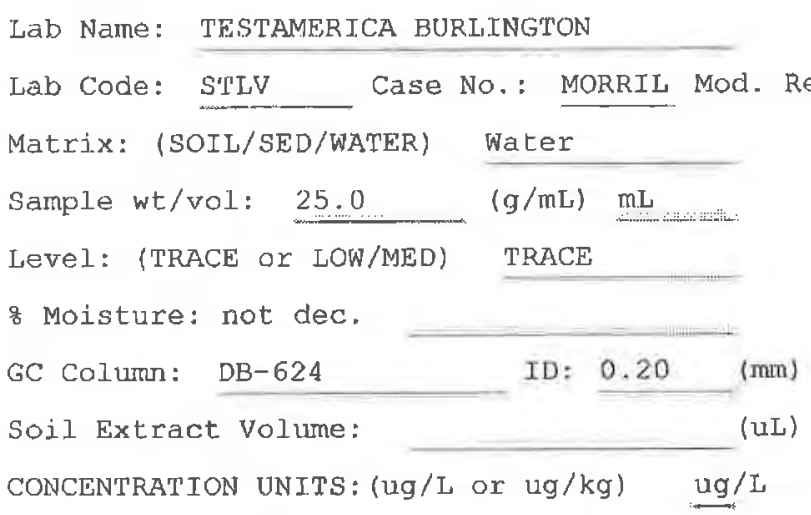

Contract: $\quad 8 \mathrm{E}-00302$

\begin{tabular}{|l|l|r|r|r|r|}
\hline \multicolumn{1}{|c|}{ CAS NUMBER } & \multicolumn{1}{|c|}{ COMPOUND NAME } & RT & EST. CONC. & Q \\
\hline & Unknown & 6.67 & 3.2 & B X J \\
\hline E9667961 & Total Alkanes & N/A & \\
\hline
\end{tabular}

1 EPA-designated Registry Number.

Lab Sample ID $\$$ 200-7357-6

Lab Fi.ie ID: DHTA11.D

Date Received:

Date Analyzed: 10/07/2011

Dilution Factor: 1.0

Soil. Aliquot Volume:

Purge Volume: 25.0 (mL)

01

02 
$1 A$ - FORM I VOA-1

VOLATILE ORGANICS ANALYSIS DATA SHEET
EPA SAMPLE NO.

VIBLKDM

Lab Name: TESTAMERICA BURL.INGTON

Contract: $\quad 8 \mathrm{E}-00302$

Lab Cocle: STLV Case No.: MORRIL Mod, Ref No: :

SDG No.: 200-7357

Matrix: (SOIJ,/SED/WATER)

Sample wt/vol: 25.0

Water

$(g / m L) \quad m L$

Level: (TRACE/LOW/MED) TRACE

\& Moisture: not dec.

GC Column: DB-624

ID : 0.20

(mm)

Soil Extract Volume:

(uL)

(mL)

Purge Volume: 25.0
Lab Sample ID: VIBLK 200-26380/9

Lab File ID: DHTA09.D

Date Received:

Date Analyzed: 10/07/2011

Dilution Factor: 1.0

Soil Aliquot volume:

\begin{tabular}{|c|c|c|c|}
\hline CAS NO. & COMPOUND & $\begin{array}{l}\text { CONCENTRATION UNITS: } \\
(\mathrm{ug} / \mathrm{L} \text { or } \mathrm{ug} / \mathrm{kg}) \mathrm{ug} / \mathrm{L}\end{array}$ & $Q$ \\
\hline $75-71-8$ & Dichlorodifluoromethane & 0.50 & $\mathrm{U}$ \\
\hline $74-87-3$ & Chloromethane & 0.50 & U \\
\hline $75-01-4$ & Vinyl chloride & 0.50 & $\mathrm{u}$ \\
\hline $74-83-9$ & Bromomethane & 0.50 & $\mathrm{U}$ \\
\hline $75-00-3$ & Chloroethane & 0.50 & $\mathrm{U}$ \\
\hline $75-69-4$ & Trichlorofluoromethane & 0.50 & $\mathrm{U}$ \\
\hline $75-35-4$ & 1,1-Dichlorcethene & 0.50 & $\mathrm{U}$ \\
\hline $76-13-1$ & 1, 1,2 -Trichloro-1,2,2-trifluoroethane & 0.50 & $\mathrm{U}$ \\
\hline $67-64-1$ & Acetone & 5.0 & $\mathrm{U}$ \\
\hline $75-15-0$ & Carbon disulfide & 0.027 & $\mathrm{JB}$ \\
\hline $79-20-9$ & Nethyl acetate & 0.50 & 0 \\
\hline $75-09-2$ & Methylene Chloride & 0.50 & $\mathrm{u}$ \\
\hline $156-60-5$ & trans-1,2-Dichloroethene & 0.50 & $\mathrm{U}$ \\
\hline $1634-04-4$ & Methyl tert-butyl ether & 0.50 & $\mathrm{U}$ \\
\hline $75-34-3$ & 1,1-Dichloroethane & 0.50 & $\mathrm{U}$ \\
\hline $156-59-2$ & cis-1,2-Dichloroetherie & 0.50 & $\mathrm{U}$ \\
\hline $78-93-3$ & 2-Butanone & 5.0 & $\mathrm{U}$ \\
\hline $74-97-5$ & Bromochloromethane & 0.50 & $\mathrm{U}$ \\
\hline $67-66-3$ & Chloroform & 0.50 & $\mathrm{U}$ \\
\hline $71-55-6$ & 1,1,1-Trichloroethane & 0.50 & $\mathrm{U}$ \\
\hline $110-82-7$ & Cyclohexane & 0.50 & $\mathrm{U}$ \\
\hline $56-23-5$ & Carbon tetxachlorjde & 0.022 & J $B$ \\
\hline 7$]-43-2$ & Benzene & 0.50 & $\mathrm{U}$ \\
\hline $107-06-2$ & 1,2-Dichloroethane & 0.50 & $\mathrm{U}$ \\
\hline
\end{tabular}

Report 1,4-Dioxane for Low-Medium VOA analysis only 
EPA SAMPLE NO.

VTBI,KDM
Lab Name: TESTAMFRICA BURLINGTON

lab Code: STLV Case No.: MORRIL Mod. Ref No.:

Matrix: (SOIL/SED/WAPER) Water

Sample wt/vol: 25.0

Level: (TRACE/IOW/MED

( $\mathrm{g} / \mathrm{mL}) \mathrm{mis}$

g Moisture: not dec.

GC Column: DB-62.4

TD : 0.20

Soil Extract volume:

PRACE

Purge volume: 25.0
Contract: $\quad 8 E-00302$

SDG No.: $200-7357$

Lab Sample ID: VIBLK 200-26380/9

Lab File ID: OHTA09.D

Date Received:

Date Aralyzed: $10 / 07 / 2011$

Dilution Factor: 1.0

Soil Aliquot volume:

\begin{tabular}{|c|c|c|c|}
\hline CAS NO. & COMPOUND & $\begin{array}{l}\text { CONCENTRATION UNITS: } \\
(\mathrm{ug} / \mathrm{L} \text { or } \mathrm{ug} / \mathrm{kg} \text { ) ug/I }\end{array}$ & $Q$ \\
\hline $79-.01-6$ & Trichloroethene & 0.50 & $\mathrm{U}$ \\
\hline $108-87-2$ & Methylcyclohexane & 0.50 & $\mathrm{U}$ \\
\hline $78-87-5$ & 1,2-Dichloropropane & 0.50 & $\mathrm{U}$ \\
\hline $75-27-4$ & Bromodichloromethane & 0.50 & $\mathrm{U}$ \\
\hline $10061-01-5$ & cis-1,3-Dichloropropene & 0.50 & $\mathrm{U}$ \\
\hline $108-10-1$ & 4-Methyl-2-pentanone & 5.0 & $\mathrm{U}$ \\
\hline $108-88-3$ & Toluene & 0.0043 & $\mathrm{JB}$ \\
\hline $10061-02-6$ & trans-1,3-Dichloropropene & 0.50 & $\mathrm{U}$ \\
\hline $79-00-5$ & 1,1,2-Trichloroethane & 0.50 & $\mathrm{U}$ \\
\hline $127-18-4$ & Tetrachloroethene & 0.50 & $\mathrm{U}$ \\
\hline $591-78-6$ & 2-Hexanone & 5.0 & $\mathrm{U}$ \\
\hline $124-48-1$ & Dibromochloromethane & 0.50 & $\mathrm{U}$ \\
\hline $106-93-4$ & 1,2-Dibromoethane & 0.50 & $\mathrm{U}$ \\
\hline $108-90-7$ & Chlorobenzene & 0.50 & $\mathrm{U}$ \\
\hline $100-41-4$ & Ethylbenzene & 0.50 & $\mathrm{U}$ \\
\hline $95+47-6$ & o-Xylene & 0.50 & $\mathrm{U}$ \\
\hline $179601-23-1$ & Jn, $\mathrm{p}-\mathrm{xy}$ lene & 0.50 & $\mathrm{U}$ \\
\hline $100-42-5$ & Styrene & 0.50 & $\mathrm{U}$ \\
\hline $75-25-2$ & Bromoform & 0.50 & $\mathrm{U}$ \\
\hline $98-82-8$ & I sopropylbenzene & 0.50 & $\mathrm{U}$ \\
\hline $79-34-5$ & $1,1,2,2$-Tetrachloroethane & 0.50 & $\mathrm{U}$ \\
\hline $541-73-1$ & 1,3-Dichlorobenzene & 0.50 & $\mathrm{U}$ \\
\hline $106-46-7$ & 1,4-Dichlorobenzene & 0.50 & $\mathrm{U}$ \\
\hline $95-50-1$ & 1,2-Dichlorobenzene & 0.50 & U \\
\hline $96-12-8$ & 1,2-Dibromo-3-Chloropropane & 0.50 & $\mathrm{U}$ \\
\hline $120-82-1$ & 1,2,4-Trichlorobenzene & 0.50 & U \\
\hline $87-61-6$ & $1,2,3-T r i c h l o r o b e n z e n e$ & 0.50 & $\mathrm{U}$ \\
\hline
\end{tabular}


, $1 \mathrm{~J}$ - FORM I VOA-IIC

VOLATILE ORGANICS ANALYSIS DATA SHEET TENTATIVELY IDENTIFIED COMPOUNDS
EPA SAMPLE NO.

VIBLIKDM

Lab Name: TESTAMERICA BURLINGTON Case No.: MORRIL Mod. Ref No.

Contract: $8 \mathrm{E}-00302$

Lab Code: STLV

water $(\mathrm{g} / \mathrm{mL}) \mathrm{mL}$

Sample wt/vol: 25.0

Level: (TRACE or LOW/MED)

TRACE

a Moigture: not dec.

GC Column: $D B-624$

Soil Extract Volumes ID: $0.20 \quad$ (ImI)

(uL)
Lab Sample ID: VIBLK 200-26380/9

Lab File ID: DHTAO9.D

Date Received:

Date Analyzed: 10/07/2011

Dilution Factor: 1.0

Soil Aliquat Volume: (uL)

Purge Volume: 25.0 (mL)

$\longrightarrow(\mathrm{mL})$

CONCENTRATION UNITS: (ug./L Or $\mathrm{ug} / \mathrm{kg}) \mathrm{ug} / \mathrm{L}$

01

02

\begin{tabular}{|l|l|c|c|c|}
\hline CAS NUMBER & \multicolumn{1}{|c|}{ COMPOUND NAME } & RT & EST. CONC. & Q \\
\hline & Unknown & 6.67 & 3.0 & $\mathrm{~B} \times \mathrm{J}$ \\
\hline E.966796 & Total Alkanes & N/A & & \\
\hline
\end{tabular}

1 EPA-desjgnated Registry Number. 
Argonne

\section{Environmental Science Division}

Argonne National Laboratory

9700 South Cass Avenue, Bldg. 203

Argonne, IL 60439-4843

www.anl.gov 